\title{
Power and Area Efficient Sub-threshold 6T SRAM with Horizontal Local Bit-Lines and Bit-Interleaving
}

\author{
by \\ Sukneet Basuta, B.Eng. \\ A thesis submitted to the \\ Faculty of Graduate and Postdoctoral Affairs \\ in partial fulfillment of the requirements for the degree of
}

Master of Applied Science in Electrical \& Computer Engineering

Ottawa-Carleton Institute for Electrical and Computer Engineering

Department of Electronics

Carleton University

Ottawa, Ontario

January, 2015

(C) Copyright

Sukneet Basuta, 2015 


\section{Abstract}

SRAM in the typical microprocessor consumes a substantial amount of on-chip area and significantly contributes to static power dissipation. Previous studies have shown that sub-threshold operation presents a minimum energy point that is optimal if ultralow power consumption is desired. However, the standard 6T SRAM cell does not operate at sub-threshold voltages. Instead, designs with higher transistor counts are typically used for sub-threshold operation. These designs generally have low integration density.

This study presents a new SRAM architecture with minimum area that utilizes a modified 6T SRAM cell for sub- and near-threshold operation in ultra-low power applications. This new architecture introduces horizontal bit-lines, mitigates halfselect disturb, and supports bit-interleaving. The proposed design's stability was thoroughly tested in the presence of process, temperature, and voltage variations, and compared to the standard 6T and traditional 8T cells. A 32kb SRAM block implementing the proposed architecture was designed, simulated, and contrasted to a traditional 8T SRAM cell block.

The simulated $32 \mathrm{~kb}$ SRAM block operates at a maximum frequency of $544.8 \mathrm{khz}$ and 6.70 Mhz for the read and write operations, respectively, and consumes 0.586 $\mathrm{pJ} /$ bit in the read operation and $0.17 \mathrm{pJ} /$ bit in the write operation. A very similar $32 \mathrm{~kb}$ SRAM block consisting of the traditional 8T SRAM cell was found to have a maximum frequency of $544.8 \mathrm{kHz}$ and $2.89 \mathrm{Mhz}$ for the read and write operations, respectively, and consumes $0.736 \mathrm{pJ} /$ bit in the read operation and $0.205 \mathrm{pJ} / \mathrm{bit}$ in the write operation. The results show that the proposed design has lower power consumption than the 8T SRAM block, comparable read performance, and better write performance. This was all achieved while only having a $10 \%$ increase in area per bit over the conventional $6 \mathrm{~T}$ thin-cell layout. 


\section{Acknowledgments}

I would like to thank

Professor Maitham Shams, my thesis supervisor, for accepting me as a graduate student, his support, and guidance.

Professor Dimitrios Makrakis for his support at my brief time at the University of Ottawa.

Scott Bruce for helping with all my support problems and putting up with my incessant use of the Linux systems.

My family for their support.

All my friends for being my friends.

Lastly, I want to thank CMC Microsystems and TSMC for providing the design tools and technology kits used in this thesis. 


\section{Contents}

Abstract $\quad$ ii

Acknowledgments $\quad$ iii

Table of Contents $\quad$ iv

List of Tables vii

List of Figures viii

Nomenclature $\quad$ xvii

1 Introduction 1

1.1 Motivation . . . . . . . . . . . . . . . . . . 1

1.2 Thesis Objectives . . . . . . . . . . . . . . . . . 3

1.3 Thesis Organization . . . . . . . . . . . . . . . 3

2 SRAM Design \& Operation 5

$2.16 \mathrm{~T}$ SRAM Cell . . . . . . . . . . . . . . . . 5

2.1.1 Read Operation . . . . . . . . . . . . . 6

2.1.2 Write Operation ............... 7

2.2 Cell stability . . . . . . . . . . . . . . . . 8

2.2.1 Measuring SNM . . . . . . . . . . . . . . . . 13

2.2.2 Measuring SNM by Circuit Simulation . . . . . . . . . . 15

2.3 Low-power SRAM . . . . . . . . . . . . . . . . . . . . . . . 18

2.4 Hierarchical bit-lines . . . . . . . . . . . . . . . . . . 21

2.5 Single-ended SRAM . . . . . . . . . . . . . . . 23

2.6 Half-Select disturb . . . . . . . . . . . . . . . . 25 
2.7 Bit-interleaving . . . . . . . . . . . . . . . . . . . . 25

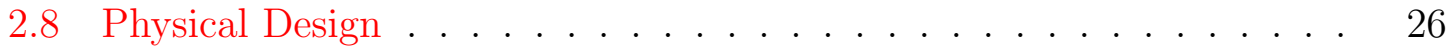

3 Literature Review $\quad 31$

3.1 A Variation-Tolerant Sub-200 mV 6-T Subthreshold SRAM . . . . . . 32

3.2 Differential 6T Sub-threshold SRAM with Low Energy and Variability Resilient Local Assist Circuit . . . . . . . . . . . . . . . . . 33

3.3 Average-8T Differential-Sensing Subthreshold SRAM With Bit Interleaving and 1k Bits Per Bitline . . . . . . . . . . . . . . 36

3.4 A Single-Ended Disturb-Free 9T Subthreshold SRAM With CrossPoint Data-Aware Write Word-Line Structure, Negative Bit-Line, and Adaptive Read Operation Timing Tracing . . . . . . . . . . . . . . . 40

3.5 Analysis of Past Research . . . . . . . . . . . . . . . . 43

4 Proposed SRAM Architecture $\quad 45$

4.1 Write Operation . . . . . . . . . . . . . . . . . 47

4.2 Read Operation . . . . . . . . . . . . . . . . 53

4.3 Hold Operation . . . . . . . . . . . . . . . . . 66

4.4 Cell Layout . . . . . . . . . . . . . . . . . . . . 66

5 Static Noise Margin Evaluations $\quad 70$

5.1 Process Variations . . . . . . . . . . . . . . . . 70

5.1 .1 Hold Operation . . . . . . . . . . . . . . . . 71

5.1 .2 Write Operation .................. . . 72

5.1 .3 Read Operation . . . . . . . . . . . . . 76

5.2 Temperature Variations . . . . . . . . . . . . . . . . 80

5.2 .1 Hold SNM . . . . . . . . . . . . . . . . . . . . . . . 81

5.2 .2 Read SNM . . . . . . . . . . . . . . . . . . 89

5.2 .3 Write SNM . . . . . . . . . . . . . . 97

6 SRAM Block Simulations 106

6.1 SRAM Block . . . . . . . . . . . . . . . 106

6.2 SRAM Block Operation . . . . . . . . . . . . . . . 109

6.3 Comparison to 8T SRAM Block . . . . . . . . . . . . . 115

6.3.1 Write Operation ................ 118

6.3.2 Read Operation . . . . . . . . . . . . . . . 122 
6.3 .3 Idle State . . . . . . . . . . . . . . . . . . . . . . . 123

6.3.4 Frequency of Operation . . . . . . . . . . . . 126

6.4 Comparison at Various Supply Voltages . . . . . . . . . . . . . 127

6.4.1 Frequency of Operation . . . . . . . . . . . . . 128

7 Conclusion 131

7.1 Future Work . . . . . . . . . . . . . . . . . . . . . . . . 132

$\begin{array}{ll}\text { List of References } & 133\end{array}$

$\begin{array}{lll}\text { Appendix A Static Noise Margin Simulation Results } & 138\end{array}$

A.1 Hold Operation . . . . . . . . . . . . . . . . . . 138

A.2 Write Operation . . . . . . . . . . . . . . . . . 144

A.3 Read Operation . . . . . . . . . . . . . . . . . 151

$\begin{array}{ll}\text { Appendix B Copyright Permissions } & 156\end{array}$

B.1 Intel Copyright Permission . . . . . . . . . . . . . . . 156

B.2 IEEE Copyright Permission . . . . . . . . . . . . . 156 


\section{List of Tables}

6.1 Byte Layout . . . . . . . . . . . . . . . . . . . . . . 107

6.2 Summary of comparison between the Proposed SRAM block and the 8T SRAM block. . . . . . . . . . . . . . . . . . . . 127

A.1 1-cell Configuration . . . . . . . . . . . . . . . . . . 141

A.2 2-cell Configuration . . . . . . . . . . . . . . . . . . 141

A.3 4-cell Configuration . . . . . . . . . . . . . . . . . . . . . 142

A.4 8-cell Configuration . . . . . . . . . . . . . . . . . . 142

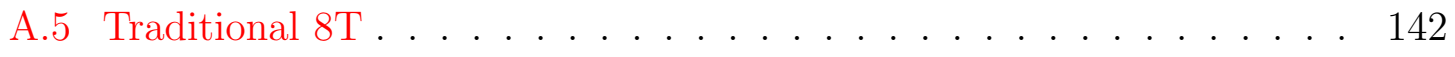

A.6 Standard 6T . . . . . . . . . . . . . . . . . . 143

A.7 1-cell Configuration . . . . . . . . . . . . . . . . . . 144

A.8 2-cell Configuration . . . . . . . . . . . . . . . . . . . . 147

A.9 4-cell Configuration . . . . . . . . . . . . . . . . . . 148

A.10 8-cell Configuration . . . . . . . . . . . . . . . . . 148

A.11 Traditional 8T . . . . . . . . . . . . . . . . . . . . 149

A.12 Traditional 8T-SE . . . . . . . . . . . . . . . . . . . . 149

A.13 Standard 6T . . . . . . . . . . . . . . . . . 150

A.14 1-cell Configuration . . . . . . . . . . . . . . . . . . 152

A.15 2-cell Configuration . . . . . . . . . . . . . . . . . . 153

A.16 4-cell Configuration . . . . . . . . . . . . . . . . . . 154

A.17 8-cell Configuration . . . . . . . . . . . . . . . . 154

A.18 Traditional 8T . . . . . . . . . . . . . . . . . 154

A.19 Standard 6T . . . . . . . . . . . . . . . . . 155 


\section{List of Figures}

1.1 Processor die map of the Intel Sandy Bridge microarchitecture [1]. (C)2011 Intel Corporation . . . . . . . . . . . . . . . . . . 2

2.1 Standard 6T SRAM cell. . . . . . . . . . . . . . . . . 5

2.2 Standard 6T SRAM cell read operation. . . . . . . . . . . . . 6

2.3 Standard 6T SRAM cell read operation timing diagram. . . . . . . . 7

2.4 Standard 6T SRAM cell write operation. . . . . . . . . . . . . . 8

2.5 Standard 6T SRAM cell write operation timing diagram. . . . . . . . 8

2.6 Butterfly diagram for the hold static noise margin (HSNM) of a standard 6T SRAM cell. . . . . . . . . . . . . . . . . 9 9

2.7 Butterfly diagrams for $6 \mathrm{~T}$ SRAM. . . . . . . . . . . . . . . 10

2.8 Test circuit for measuring the hold static noise margin (HSNM). . . . 11

2.9 WSNM butterfly diagrams for 6T SRAM. . . . . . . . . . . . . . . 12

2.10 Test circuit for measuring the read static noise margin (RSNM). . . . 13

2.11 Butterfly diagram for the read static noise margin (RSNM) for a standard 6T SRAM cell [2]. . . . . . . . . . . . . . . . . . 13

2.12 Test circuit for measuring the write static noise margin (WSNM). . . 14

2.13 Estimation of SNM in a $45^{\circ}$ rotated coordinate system [3]. . . . . . . 15

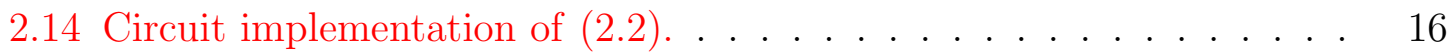

2.15 Circuit implementation of $(2.4) \ldots \ldots \ldots$. . . . . . . 17

2.16 Butterfly (SNM) curves for the hold operation transposed onto the $45^{\circ}$ rotated coordinate system $(\mathrm{u}, \mathrm{v})$. The dashed curve is $\mathrm{v}_{1}$ and the solid curve is $\mathrm{v}_{2} . \mathrm{V}_{D D}$ is $1.0 \mathrm{~V}$ in this case, so $u$ was swept from $-707 \mathrm{mV}$ to $707 \mathrm{mV}$. . . . . . . . . . . . . . . . . . . . 17

2.17 The difference between the two butterfly (SNM) curves $\left(\mathrm{v}_{1}-\mathrm{v}_{2}\right)$ in Figure 2.16. . . . . . . . . . . . . . . . . . . . . . 18

2.18 Read SNM comparision for 6T SRAM cell at different $V_{D D} \ldots \ldots$. . . 19

2.19 The traditional 8T SRAM cell. . . . . . . . . . . . . . . . 21 
2.20 Classical SRAM array architecture [4]. . . . . . . . . . . . . . . 22

2.21 Hierarchical or divided bit-line architecture [4] . . . . . . . . . . . . 23

2.22 Simplest single-ended SRAM cell architecture [5]. . . . . . . . . . . . 24

2.23 Single-ended SRAM bit-cell with different read and write paths [6].

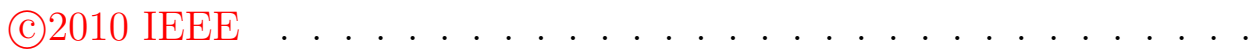

2.24 Half select disturb problem in a standard 8T SRAM cell array [7]. (C) $2010 \mathrm{IEEE}$. . . . . . . . . . . . . . . . . . . . .

2.25 SRAM word organization: the top row shows the typical case where all bits in a word are stored adjacently, unlike the bottom row that shows an implementation of bit-interleaving [8]. (C)2011 IEEE . . . . . . . . 27

2.26 Historical layout of $6 \mathrm{~T} \mathrm{SRAM}$ cell $[2] \ldots \ldots$. . . . . . . . . . . 28

2.27 Arrayed historical layout of 6T SRAM cell [2] . . . . . . . . . . . . . 29

2.28 Lithographically friendly thin cell $6 \mathrm{~T}$ cell layout [2] . . . . . . . . . . 29

2.29 Thin cell 6T cell layout showing rounding of the notch [9]. (C)2009 IEEE 30

2.30 Diffusion-notch-free, or rectangular-diffusion, layout of 6T SRAM cell. Note how all transistors are sized equally [9]. (C)2009 IEEE . . . . . . 30

3.1 SRAM Cell design and area [10]. (c)2008 IEEE . . . . . . . . . . . . . 33

3.2 Cell array structure [10]. (c)2008 IEEE . . . . . . . . . . . . . . 34

3.3 Chiou et al.'s proposed SRAM architecture [11]. (c)2013 IEEE . . . . 35

3.4 Khayatzadeh and Lian's proposed SRAM cell [12]. (C)2013 IEEE . . . 36

3.5 Khayatzadeh and Lian's proposed SRAM cell in the hold state [12]. (C) 2013 IEEE . . . . . . . . . . . . . . . . . . . . 37

3.6 Khayatzadeh and Lian's proposed SRAM cell in the read operation [12]. C 2013 IEEE . . . . . . . . . . . . . . . . . . . . . . 38

3.7 Khayatzadeh and Lian's proposed SRAM cell when half-selected [12].

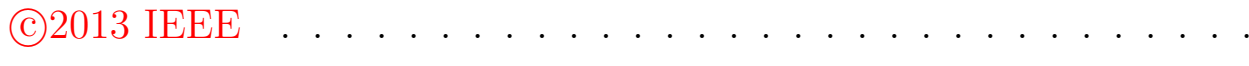

3.8 Khayatzadeh and Lian's proposed SRAM cell in the write operation

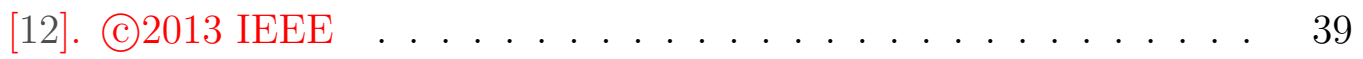

3.9 SRAM cell proposed in [13]. (c)2012 IEEE . . . . . . . . . . . . . 40

3.10 Timing diagram of the 9T SRAM cell designed in [13]. (C)2012 IEEE 41

3.11 Write operation of the 9T SRAM cell presented in [13]. (C)2012 IEEE 41

3.12 Half-selected rows during the write operation of the 9T SRAM cell presented in [13]. (c)2012 IEEE . . . . . . . . . . . . . . . . 42

4.1 Basic architecture of the proposed cell block in a 2-cell configuration. $\quad 45$ 
4.2 Schematic of the proposed cell block in a 1-cell configuration. . . . . . 46

4.3 Schematic of the proposed cell block in a 2-cell configuration. . . . . . 46

4.4 Schematic of the proposed cell block in a 4-cell configuration. . . . . . 47

4.5 Schematic of the proposed cell block in a 8-cell configuration. . . . . . 47

4.6 Write operation of the proposed SRAM design. . . . . . . . . . . . . 48

4.7 Timing diagram of the write operation when writing a ' 0 ' . . . . . . 49

4.8 Timing diagram of the write operation when writing a ' 1 ' . . . . . . 50

4.9 Comparison of the write SNM of the proposed design, the differential traditional $8 \mathrm{~T} /$ standard $6 \mathrm{~T}$ (they are same in this case), and singleended $8 \mathrm{~T}$ write schemes. The $V_{D D}$ used in the simulation is $300 \mathrm{mV}$.

4.10 Half-select disturb in the proposed design. . . . . . . . . . . . . . 52

4.11 Read operation of the proposed design when reading a ' 1 '. . . . . . . 53

4.12 Read operation of the proposed design when reading a ' 0 ' . . . . . . 54

4.13 Bit-line drainage current when a single minimum-size transistor, a single oversized transistor (2 times the minimum width), and a pair of minimum-size parallel stacked transistors (PTS [14]) are used for readdecoupling. Simulation is performed for the FS corner. . . . . . . . 55

4.14 Timing diagram of the read operation when reading a ' 1 ' . . . . . . . 56

4.15 Timing diagram of the read operation when reading a ' 0 ' . . . . . . . 57

4.16 Drain current vs. width for an NMOS transistor. The inverse narrow width effect can be observed by comparing the drain current of a minimum width transistor with one twice the size (marked on the

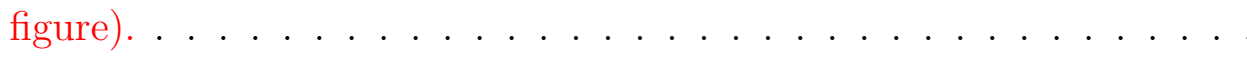

4.17 Comparison of the read current when a single minimum-size transistor, and a pair of minimum-size parallel stacked transistors (PTS) are used as read decoupling transistors when reading a ' 1 '. The read current of the traditional $8 \mathrm{~T}$ cell is included for comparison purposes. Simulation was performed for the FS corner. . . . . . . . . . . . . .

4.18 Comparison of the local bit-line voltage when a single minimum-size transistor, and a pair of minimum-size parallel stacked transistors (PTS) are used as read decoupling transistors when reading a ' 1 '. The voltage level of the data being read (Node Q) is included for comparison purposes. Simulation was performed for the FS corner. . . . . . . . . 
4.19 Comparison of the bit-line leakage for a combination of parallel readdecoupling transistors and stacked read-access transistors, a single read-decoupling transistor and stacked read-access transistors, and parallel read-decoupling transistors and a single read-access transistor. The bit-line leakage for the read path in the 8T SRAM cell is included for comparison. The simulation is performed for the FS corner. . . . . 61

4.20 NMOS transistors stacked in series. . . . . . . . . . . . . . .

4.21 Comparison of the sub-threshold current between two minimum-length series stacked NMOS transistors and one NMOS transistor with a length of two times the minimum-length. $V_{D D}$ is $300 \mathrm{mV} \ldots . .$. . .

4.22 Comparison of the read SNM of the proposed design, the traditional $8 \mathrm{~T}$, and standard $6 \mathrm{~T}$ SRAM cells. The $V_{D D}$ used in the simulation is $300 \mathrm{mV} \ldots \ldots \ldots \ldots \ldots \ldots \ldots \ldots$

4.23 Idle operation of the proposed design. . . . . . . . . . . . . .

4.24 Comparison of the hold SNM of the proposed design, the traditional $8 \mathrm{~T}$, and standard $6 \mathrm{~T}$ SRAM cells for a $V_{D D}$ of $300 \mathrm{mV}$. . . . . . .

4.25 Layout of the proposed bit cell. The layout is based on the layout of the $6 \mathrm{~T}$ thin-cell. . . . . . . . . . . . . . . . . . . 68

4.26 Layout for the overhead circuitry required for the proposed cell block, including read and write decoupling transistors, and read access transistors. . . . . . . . . . . . . . . . . . .

4.27 A section of the proposed SRAM architecture. The layout here shows 2 bit-cells with the overhead circuitry. Any additional cells would be added to the sides. . . . . . . . . . . . . . . . . . .

5.1 Simulation results for Hold SNM vs. $V_{D D}$ for the different block configurations proposed, the traditional 8T cell, and the standard 6T cell. The plotted points are $\mu-5 \sigma$. The points in the table are in $\mathrm{mV}$. . . 
5.2 Estimated probably density function of the hold operation for the 8cell block configuration and $8 \mathrm{~T} / 6 \mathrm{~T}$ SRAM cells (they are the exact same). $V_{D D}$ is $300 \mathrm{mV}$ and the temperature is $27^{\circ} \mathrm{C}$. Only the 8-cell configuration is included because all configurations have very similar distributions. This is similarly true for all sub-threshold operating voltages. Refer to Figure A.1, Figure A.2, and Figure A.3 for the histograms of the 8-cell block configuration, 8T SRAM cell, and 6T SRAM cell, respectively. . . . . . . . . . . . . . . .

5.3 Simulation results for Write SNM vs. $V_{D D}$ for the different block configurations proposed, the traditional $8 \mathrm{~T}$ cell, the traditional $8 \mathrm{~T}$ cell with single-ended write operation, and the standard 6T cell. The plotted points are $\mu-5 \sigma$. The points in the table are in $\mathrm{mV} . . . . . .$.

5.4 Estimated probably density function of the hold operation for the 8cell block configuration at various supply voltages. The temperature is $27^{\circ} \mathrm{C}$. Only the 8 -cell configuration is included because all configurations have very similar distributions. Refer to Figure A.4, Figure A.5, Figure A.6, and Figure A.7 for the histograms of the 8-cell configuration with supply voltages of $200 \mathrm{mV}, 250 \mathrm{mV}, 300 \mathrm{mV}$, and $350 \mathrm{mV}$, respectively. . . . . . . . . . . . . . . . .

5.5 Estimated probably density function of the write operation for the 8T/6T SRAM cell (they are the same), and $8 \mathrm{~T}$ single-ended write scheme. $V_{D D}$ is $300 \mathrm{mV}$ and the temperature is $27^{\circ} \mathrm{C}$. Only the the density function when the $V_{D D}$ is $300 \mathrm{mV}$ included because all supply voltages have very similar distributions. Refer to Figure A.8, and Figure A.9 for the histograms of the single-ended 8T, and 8T/6T SRAM cells, respectively. . . . . . . . . . . . . . . . . . . . 77

5.6 Simulation results for Read SNM vs. $V_{D D}$ for the different block configurations proposed, the traditional $8 \mathrm{~T}$ cell, and the standard $6 \mathrm{~T}$ cell. The plotted points are $\mu-5 \sigma$. The points in the table are in $\mathrm{mV}$. . . 
5.7 Estimated probably density function of the read operation for the 8-cell block configuration, $8 \mathrm{~T}$, and $6 \mathrm{~T}$ SRAM cells. $V_{D D}$ is $300 \mathrm{mV}$ and the temperature is $27^{\circ} \mathrm{C}$. Only the 8-cell configuration is included because all configurations have very similar distributions. This is similarly true for all sub-threshold operating voltages. Refer to Figure A.10, Figure A.11, and Figure A.12 for the histograms of the 8-cell block configuration, 8T SRAM cell, and 6T SRAM cell, respectively. . . . . 79

5.8 Hold SNM vs. Temperature $\left({ }^{\circ} \mathrm{C}\right)$ for the 1-cell block. . . . . . . . . . 82

5.9 Hold SNM vs. Temperature $\left({ }^{\circ} \mathrm{C}\right)$ for the 2-cell block. . . . . . . . . . 83

5.10 Hold SNM vs. Temperature $\left({ }^{\circ} \mathrm{C}\right)$ for the 4-cell block. . . . . . . . . . 84

5.11 Hold SNM vs. Temperature $\left({ }^{\circ} \mathrm{C}\right)$ for the 8-cell block. . . . . . . . . . 85

5.12 Hold SNM vs. Temperature $\left({ }^{\circ} \mathrm{C}\right)$ for the traditional 8T SRAM cell. . 86

5.13 Hold SNM vs. Temperature $\left({ }^{\circ} \mathrm{C}\right)$ for the standard 6 T SRAM cell. . . 87

5.14 Simulation results for Hold SNM vs. Temperature $\left({ }^{\circ} \mathrm{C}\right)$ for the different block configurations proposed, the traditional $8 \mathrm{~T}$ cell, and the standard $6 \mathrm{~T}$ cell. $V_{D D}$ is $300 \mathrm{mV}$. The plotted points are $\mu-5 \sigma$. . . . . . 88

5.15 Read SNM vs. Temperature $\left({ }^{\circ} \mathrm{C}\right)$ for the 1-cell block. . . . . . . . . . 90

5.16 Read SNM vs. Temperature $\left({ }^{\circ} \mathrm{C}\right)$ for the 2-cell block. . . . . . . . . . 91

5.17 Read SNM vs. Temperature $\left({ }^{\circ} \mathrm{C}\right)$ for the 4-cell block. . . . . . . . . . 92

5.18 Read SNM vs. Temperature $\left({ }^{\circ} \mathrm{C}\right)$ for the 8-cell block. . . . . . . . . . 93

5.19 Read SNM vs. Temperature $\left({ }^{\circ} \mathrm{C}\right)$ for the traditional 8T SRAM cell. . 94

5.20 Read SNM vs. Temperature $\left({ }^{\circ} \mathrm{C}\right)$ for the standard $6 \mathrm{~T}$ SRAM cell. Note that the y-axis has been translated down $20 \mathrm{mV}$ to show the standard deviation without changing the scale. . . . . . . . . . . . 95

5.21 Simulation results for Read SNM vs. Temperature $\left({ }^{\circ} \mathrm{C}\right)$ for the different block configurations proposed, the traditional $8 \mathrm{~T}$ cell, and the standard 6T cell. $V_{D D}$ is $300 \mathrm{mV}$. The plotted points are $\mu-5 \sigma$. . . . . . . 96

5.22 Write SNM vs. Temperature $\left({ }^{\circ} \mathrm{C}\right)$ for the 1-cell block. . . . . . . . . . 98

5.23 Write SNM vs. Temperature $\left({ }^{\circ} \mathrm{C}\right)$ for the 2-cell block. . . . . . . . . . 99

5.24 Write SNM vs. Temperature $\left({ }^{\circ} \mathrm{C}\right)$ for the 4-cell block. . . . . . . . . . 100

5.25 Write SNM vs. Temperature $\left({ }^{\circ} \mathrm{C}\right)$ for the 8-cell block. . . . . . . . . . 101

5.26 Write SNM vs. Temperature $\left({ }^{\circ} \mathrm{C}\right)$ for the traditional 8T SRAM cell. . 102

5.27 Write SNM vs. Temperature $\left({ }^{\circ} \mathrm{C}\right)$ for the single-ended write scheme utilizing the traditional 8T SRAM cell. . . . . . . . . . . . . 103 
5.28 Write SNM vs. Temperature $\left({ }^{\circ} \mathrm{C}\right)$ for the traditional 8T SRAM cell. . 104

5.29 Simulation results for Write SNM vs. Temperature $\left({ }^{\circ} \mathrm{C}\right)$ for the different block configurations proposed, the traditional 8T cell, the singleended $8 \mathrm{~T}$ write-scheme, and the standard $6 \mathrm{~T}$ cell. $V_{D D}$ is $300 \mathrm{mV}$. The

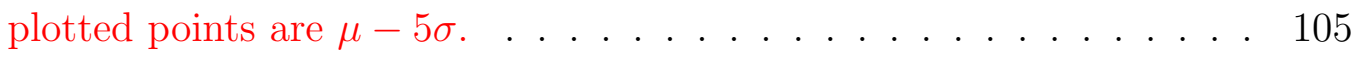

6.1 Array level block diagram of the $32 \mathrm{~kb}$ memory block for the proposed SRAM architecture. . . . . . . . . . . . . . . . . . . 108

6.2 Array level block diagram of the $32 \mathrm{~kb}$ memory block for the traditional 8T SRAM cell.

6.3 Simulated signals involved in the write operation of the $8 \mathrm{~T}$ block for FS corner at $27^{\circ} \mathrm{C}$. . . . . . . . . . . . . . . . . . . . . . 110

6.4 The problem with the write operation in the $8 \mathrm{~T}$ block. Simulation performed for FS corner at $27^{\circ} \mathrm{C}$. . . . . . . . . . . . . . . . . 112

6.5 The signals involved the write operation in the proposed block. Simulation performed for FS corner at $27^{\circ} \mathrm{C}$. . . . . . . . . . .

6.6 The signals involved the read operation in the $8 \mathrm{~T}$ block. Simulation performed for FS corner at $27^{\circ} \mathrm{C}$. Note that the reason for the spike of the RWL signal in the beginning of the read operation is due to the slowness of the decoder. . . . . . . . . . . . . . . . . . . . 114

6.7 The signals involved the read operation in the proposed block. Simulation performed for FS corner at $27^{\circ} \mathrm{C}$. Note that the spikes in the signals in the beginning of the idle state is due to changing the signals at the same time as the clock switches low. It takes time for the decoder to process the low clock state. . . . . . . . . . . . . . . 116

6.8 Comparison of the power consumption during the start-up, write, idle, and read operations. . . . . . . . . . . . . . . . . 117

6.9 Comparison of the power consumption during a write. Close up view of Figure 6.8. . . . . . . . . . . . . . . . . . . . . . . . . . . . . . 118

6.10 Comparison of the energy consumed during a write. . . . . . . . . . . 119

6.11 More accurate comparison of the power consumption during a write. . 120

6.12 More accurate comparison of the energy consumption during a write.

6.13 Comparison of the power consumption during a read. Close up view of Figure 6.8. . . . . . . . . . . . . . . . . . . . . . . . . . . . . 123

6.14 Comparison of the energy consumed during a read. . . . . . . . . . . 124 
6.15 Comparison of the power consumption during the idle state, i.e. static power consumption. Close up view of Figure 6.8. . . . . . . . . . . . 125

6.16 Comparison of the energy consumed during the idle state. . . . . . . 126

6.17 Comparison of the power consumption during the write, read, and idle operations. Simulations performed for FS corner at $27^{\circ} \mathrm{C}$. . . . . . 128

6.18 Comparison of the energy per bit consumed during the write and read operations. Simulations performed for FS corner at $27^{\circ} \mathrm{C}$. . . . . . .

6.19 Comparison of the maximum write and read speed. Simulations performed for FS corner at $27^{\circ} \mathrm{C}$. . . . . . . . . . . . . . . . . . . . 129

6.20 Comparison of the maximum write and read speed not including the decoder delay. Simulations performed for FS corner at $27^{\circ} \mathrm{C}$. . . . .

A.1 Histogram for the hold operation of the 8-cell block configuration. $V_{D D}$ is $300 \mathrm{mV}$ and the temperature is $27^{\circ} \mathrm{C}$. Only the 8 -cell configuration is included because all configurations have very similar distributions. This is similarly true for all sub-threshold operating voltages. . . . . .

A.2 Histogram for the hold operation of the traditional $8 \mathrm{~T}$ cell. $V_{D D}$ is $300 \mathrm{mV}$ and the temperature is $27^{\circ} \mathrm{C}$. . . . . . . . . . . . . 139

A.3 Histogram for the hold operation of the standard $6 \mathrm{~T}$ cell. $V_{D D}$ is $300 \mathrm{mV}$ and the temperature is $27^{\circ} \mathrm{C}$. . . . . . . . . . . . . . 140

A.4 Histogram for the write operation of the 8-cell block configuration. $V_{D D}$ is $200 \mathrm{mV}$ and the temperature is $27^{\circ} \mathrm{C}$. . . . . . . . . . . . . . 144

A.5 Histogram for the write operation of the 8-cell block configuration. $V_{D D}$ is $250 \mathrm{mV}$ and the temperature is $27^{\circ} \mathrm{C}$. . . . . . . . . . . . 145

A.6 Histogram for the write operation of the 8-cell block configuration. $V_{D D}$ is $300 \mathrm{mV}$ and the temperature is $27^{\circ} \mathrm{C}$. . . . . . . . . . . . 145

A.7 Histogram for the write operation of the 8-cell block configuration. $V_{D D}$ is $350 \mathrm{mV}$ and the temperature is $27^{\circ} \mathrm{C}$. . . . . . . . . . . 146

A.8 Histogram for the single-ended write operation of the traditional $8 \mathrm{~T}$ cell. $V_{D D}$ is $300 \mathrm{mV}$ and the temperature is $27^{\circ} \mathrm{C}$. . . . . . . . . 146

A.9 Histogram for the write operation of the traditional $8 \mathrm{~T} /$ standard $6 \mathrm{~T}$ cell. $V_{D D}$ is $300 \mathrm{mV}$ and the temperature is $27^{\circ} \mathrm{C}$. . . . . . . . . 147 
A.10 Histogram for the read operation of the 8-cell block configuration. $V_{D D}$ is $300 \mathrm{mV}$ and the temperature is $27^{\circ} \mathrm{C}$. Only the 8 -cell configuration is included because all configurations have very similar distributions. This is similarly true for all sub-threshold operating voltages. . . . . . 151

A.11 Histogram for the read operation of the traditional $8 \mathrm{~T}$ cell. $V_{D D}$ is $300 \mathrm{mV}$ and the temperature is $27^{\circ} \mathrm{C}$. . . . . . . . . . . . 152

A.12 Histogram for the read operation of the standard 6T cell. $V_{D D}$ is 300 $\mathrm{mV}$ and the temperature is $27^{\circ} \mathrm{C} \ldots \ldots \ldots \ldots$ 


\section{Nomenclature}

\section{List of Acronyms}

\begin{tabular}{|c|c|}
\hline Acronym & Definition \\
\hline BL & Bit-line \\
\hline$\overline{B L} / \mathrm{BLB}$ & Bit-line compliment \\
\hline CLK & Clock \\
\hline CMOS & Complementary metal-oxide-semiconductor \\
\hline FS & Fast NMOS, Slow PMOS \\
\hline GBL & Global bit-line \\
\hline GND & Ground \\
\hline HSNM & Hold static noise margin \\
\hline HVT & High threshold voltage \\
\hline LBL & Local bit-line \\
\hline LP & Low-power transistor variant \\
\hline MOSFET & Metal-oxide-semiconductor field-effect transistor \\
\hline NMOS & n-channel MOSFET \\
\hline PMOS & p-channel MOSFET \\
\hline PTS & Parallel stacked transistors \\
\hline PVT & Process-voltage-temperature \\
\hline RBL & Read bit-line \\
\hline
\end{tabular}




\begin{tabular}{cc} 
RSNM & Read static noise margin \\
RWL & Read word-line \\
SNM & Static noise margin \\
SRAM & Static Random Access Memory \\
TSMC & Taiwan Semiconductor Manufacturing Company Limited \\
VLSI & Very-large-scale integration \\
VSS & Source supply \\
VVSS & Virtual source supply \\
WBL & Write bit-line \\
\hline WBL & Write bit-line compliment \\
WL & Word-line \\
WSNM & Write static noise margin \\
WLA & Word-line A \\
WLB & Word-line B \\
WWL & Write word-line \\
\hline
\end{tabular}


List of Symbols

\begin{tabular}{|c|c|}
\hline Symbol & Definition \\
\hline $\mathrm{C}$ & Capacitance \\
\hline $\mathrm{I}$ & Current \\
\hline$I_{O F F}$ & Drain-source current when MOSFET is off \\
\hline$I_{O N}$ & Drain-source current when MOSFET is on \\
\hline $\mathrm{P}$ & Power consumption \\
\hline$M_{a c}$ & Access transistors \\
\hline$M_{r d}$ & Read decoupling transistors \\
\hline$M_{\text {rac }}$ & Read access transistors \\
\hline$M_{w r}$ & Write decoupling transistors \\
\hline$M_{p d}$ & Block mask transistors \\
\hline $\mathrm{N}$ & Number of \\
\hline $\mathrm{R}$ & Resistance \\
\hline $\mathrm{V}$ & Voltage \\
\hline$V_{D D}$ & Supply voltage \\
\hline$V_{t h}$ & Threshold voltage \\
\hline$V_{t n}$ & NMOS threshold voltage \\
\hline
\end{tabular}




\section{Chapter 1}

\section{Introduction}

Embedded systems such as biomedical implants, and micro-sensor networks require ultra-low power circuits to extend battery life for as long as possible. It is widely regarded that the best way to reduce power in digital circuits is to lower the supply voltage; studies have shown that the minimum energy point is formed below the threshold voltage, in the sub-threshold region [15]. While ultra-low-voltage logic design has been well researched [16], there has been considerably fewer studies on sub-threshold SRAM design. Although there has been a number of papers published on sub-threshold SRAM in the past decade, sub-threshold SRAM design remains a challenge.

Scaling down the voltage permits the grasping of a minimum energy point, but adversely affects speed and leads to large process, voltage, and temperature (PVT) variations. Additionally, the increasingly poor $I_{O N} / I_{O F F}$ ratio in the transistors as the supply voltage decreases only adds another obstacle to the challenge. With an increase in demand for ultra low-power embedded systems due to rapid advancements in CMOS technology, this problem needs to be addressed.

\subsection{Motivation}

In the past two decades, the 6T SRAM cell has been the standard for SRAM. It is, arguably, the paragon comprise of stability, performance, power consumption, and area utilization in super-threshold operation. When the voltage scales down to sub or near-threshold operation, however, the standard 6T cell has poor stability [17].

To circumvent this problem, manufacturers have been moving to the traditional 8T SRAM cell for low-voltage operation. The 8T SRAM addresses the decreased 
stability, but it incurs an area penalty of at least $30 \%$. This increase in area is a substantial issue since the on-die cache can consume a significant amount of on-chip area (see Figure 1.1). In their latest processors, Intel has moved to using the 8T cell for the fast L1 and L2 caches to increase performance at lower voltages while decreasing power consumption. However, the L3 cache is so large that utilizing the $8 \mathrm{~T}$ cell would result in significant cost and area increase. Thus, to keep using the $6 \mathrm{~T}$ SRAM cell in the L3 cache, the L3 cache and everything else off-core were given its own voltage plane and operates at a reduced speed. While this is not an example of an ultra-low power embedded system, the same problem applies.

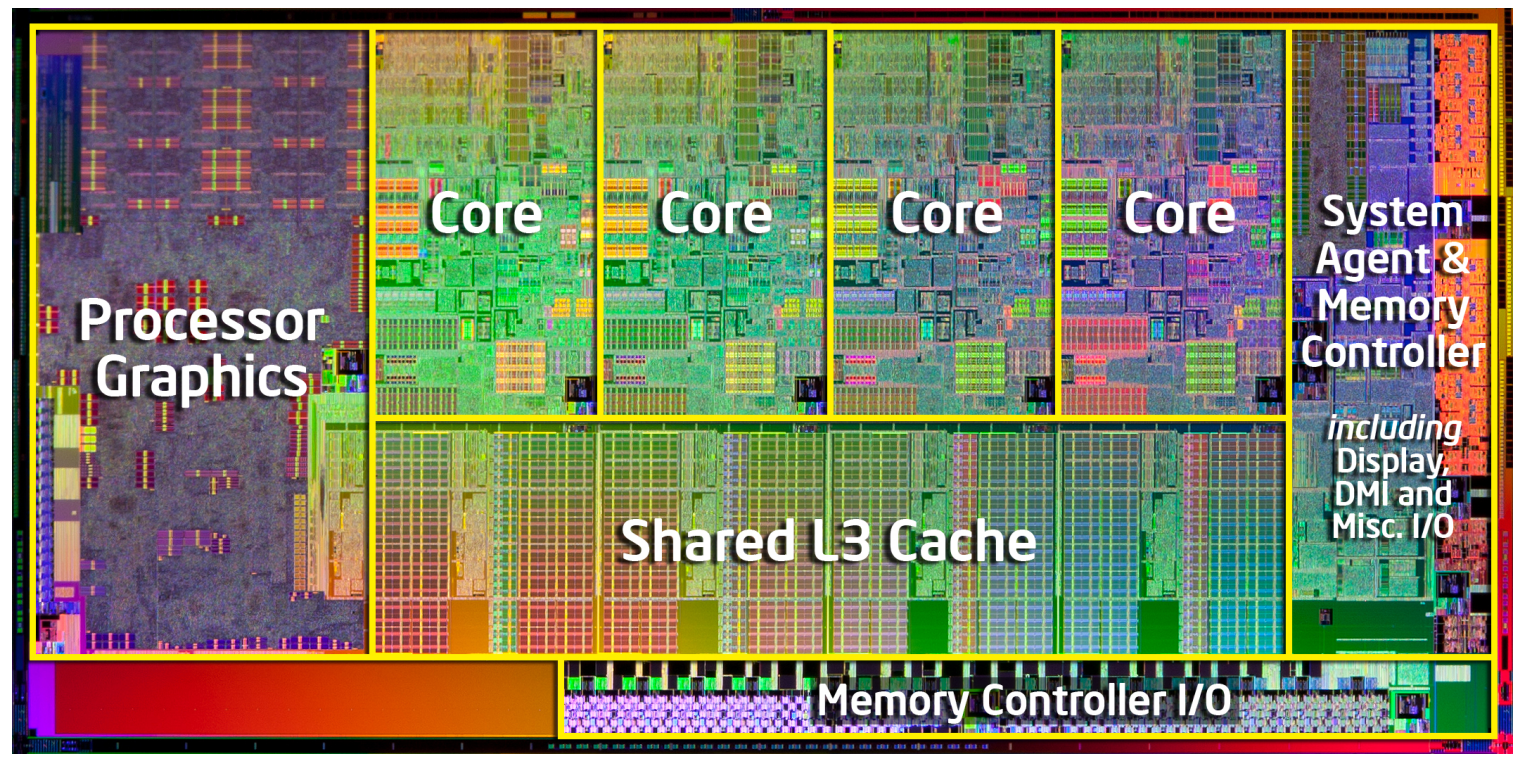

Figure 1.1: Processor die map of the Intel Sandy Bridge microarchitecture [1]. (C) 2011 Intel Corporation

Dynamic RAM (DRAM) may seem like a solution to the area problem, since the basic circuit is just one transistor with one capacitor and it's a volatile memory. Unfortunately, DRAM is generally slower than the all transistor SRAM and has to be refreshed periodically, resulting in a higher power consumption, making it unsuitable for use in caches. Non-volatile memory, such as ROMs and NVRAM, have very low power consumption, but do not have the speed to be suitable for caches and have a high area penalty. Their main purpose is for storing data when power is removed. Thus, there is no suitable replacement for SRAM.

A decent number of studies have been published in the past decade to solve the 
ultra-low voltage SRAM problem, but very few focus on minimizing area. Instead, they tend to focus on achieving lower voltage operation, increased performance, and/or increased stability. These are all important issues that need to be solved for certain ultra low-voltage applications, but many other applications would benefit more from a less costly design. Thus, to find a solution to this problem, this study will try to design a sub-threshold SRAM bit cell that minimizes area while having acceptable performance and stability.

\subsection{Thesis Objectives}

Given the above motivation, the objectives of this research are as follows:

1. Propose a sub-threshold SRAM design that minimizes area.

2. Determine the performance and stability of the SRAM design with respect to voltage-scalability, process-variations, and temperature variations.

3. Compare the proposed design to the standard 6T and traditional 8T SRAM cells to determine its suitability as an alternative prospect at sub-threshold voltages.

4. Develop a SRAM block utilizing the proposed design to ensure proper operation.

5. Evaluate the constructed SRAM block, and compare it to a similar SRAM block consisting of the traditional 8T SRAM bit cell, with emphasis on power consumption.

\subsection{Thesis Organization}

Following this introductory chapter, Chapter 2 introduces the background knowledge needed to understand this study. The basic SRAM cell operation, cell stability, lowpower SRAM, and SRAM design challenges are all tackled in this chapter.

Chapter 3 presents a review of previous studies that directly inspired this research. How each design functions, their advantages, and their problems are all summarized.

Chapter 4 introduces a new SRAM cell design. The reasoning for each aspect of the design is outlined and the operation of the design is discussed in detail. 
Chapter 5 shows the simulation results of testing the stability of the proposed SRAM cell. The effects of process, temperature, and voltage variations are all explored to provide good insight on how stable the cell is.

In Chapter 6, an entire SRAM block is simulated, utilizing the proposed cell. Its performance, power, and energy consumption are compared to the traditional $8 \mathrm{~T}$ SRAM block that is built using a very similar architecture. The metrics are then measured against various supply voltages.

Chapter 7 concludes this thesis and presents ideas for future work. 


\section{Chapter 2}

\section{SRAM Design \& Operation}

\section{$2.1 \quad 6 \mathrm{~T}$ SRAM Cell}

The basic requirements of a SRAM cell is to be able to read and write data, and to hold the data for as long as there is power. To meet these requirements, all memory cells consist of a storage cell and transfer (or access) gates. The storage cell holds the data. The transfer gates allow data to be read from and written to the storage cell. A basic flip-flop meets these demands but does so at a large size.

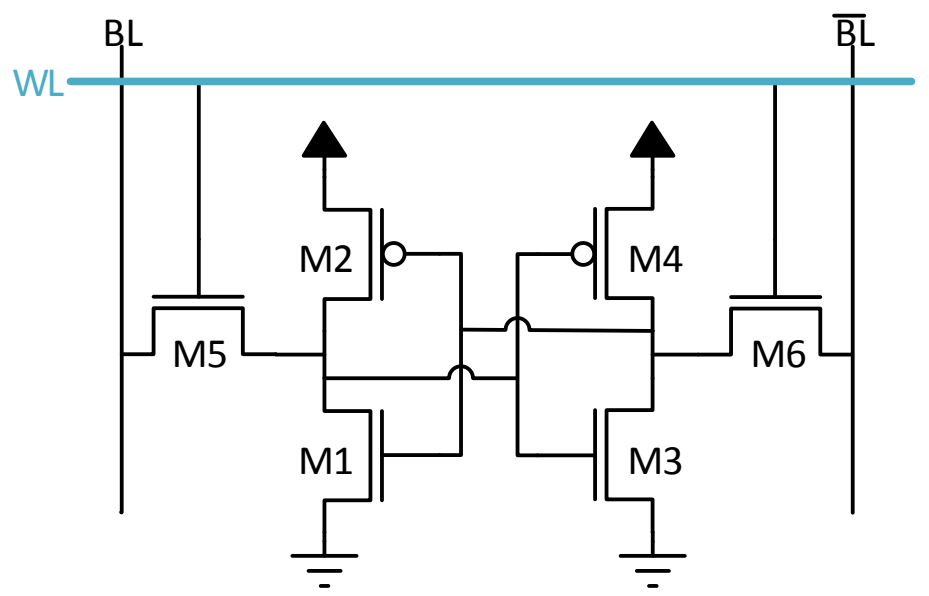

Figure 2.1: Standard 6T SRAM cell.

The standard 6T SRAM cell, illustrated in Figure 2.1, is considerably smaller than a flip-flop. This area reduction results in a more complex operation when reading and writing. However, given that SRAM dominates the area in modern microprocessors, it is a much welcomed trade-off. 
In the standard 6T SRAM, the storage cell is comprised of a pair of weak inverters (M1 and M2, M3 and M4) placed back-to-back. The positive feedback created by cross-coupling the inverters corrects disturbances caused by noise and leakage. The NMOS transistors (M1 and M3) are known as the drive or pull-down transistors, and the PMOS transistors (M2 and M4) are known as the load or pull-up transistors. The transfer gates consist of transistors M5 and M6-the access transistors [18].

When the access transistors are off (i.e. WL is low), the SRAM cell is in the hold state. The back-to-back inverters keep the state of the cell through positive feedback.

\subsubsection{Read Operation}

Figure 2.2 shows an SRAM cell being read. Before the read operation occurs, the bit-lines (BL and $\overline{\mathrm{BL}}$ ) are precharged high. When the read operation starts, the bit-lines are left floating high and the Word Line is raised high, enabling the access transistors. As a result, one access transistor will have no potential difference across it, while the other will have a potential difference of $V_{D D}$ causing current to flow through it. In the case of Figure 2.2, this causes $\overline{\mathrm{BL}}$ to be pulled down through the driver and access transistors. While $\overline{\mathrm{BL}}$ is being discharged, it will be read as a logic ' 0 ' by the peripheral circuitry. It is important to note that due to the large number of cells on each bit-line, there is a relatively large capacitance that must be discharged through the access and drive transistor. Thus, the bit-line is rarely fully discharged to a logic level '0' [18].

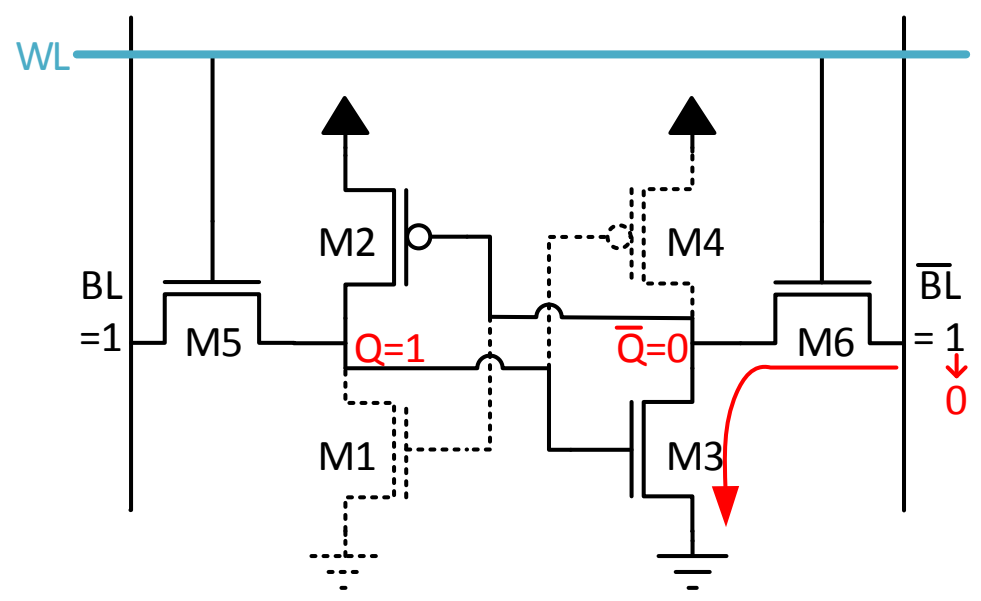

Figure 2.2: Standard 6T SRAM cell read operation. 
While $\overline{\mathrm{BL}}$ is being pulled down, node $\overline{\mathrm{Q}}$ tends to rise due to the current flowing in from M6, but is still held low by M3 (refer to Figure 2.3). Therefore, it is important to ensure the drive and access transistors are sized so that node $\overline{\mathrm{Q}}$ remains below the switching threshold of the M1/M2 inverter. In many processes, similarly sized transistors are sufficient to ensure this. However, using equal sized transistors reduces the read margin [2].

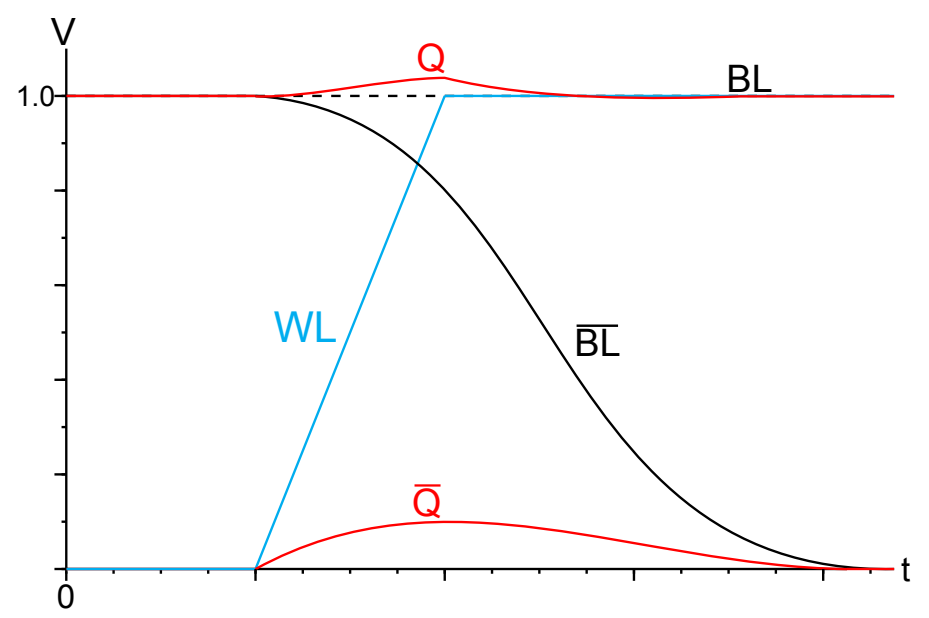

Figure 2.3: Standard 6T SRAM cell read operation timing diagram.

\subsubsection{Write Operation}

Figure 2.4 shows an SRAM cell being written to. In the standard 6T SRAM cell, data is written into the cell by writing a ' 0 ' into one node of the storage cell. The internal feedback of the cell sets the value of the other node to ' 1 '. Due to the constraint of sizing the pull-down and access transistors so that $\mathrm{Q}$ ( or $\overline{\mathrm{Q}}$ ) remains below the switching threshold during reads, it is not possible to force a node to ' 1 ' without feedback.

Once again, the bit-lines (BL and $\overline{\mathrm{BL}}$ ) are precharged high before the operation begins. When the write operation starts, the bit-line connected to the node where ' 0 ' is desired to be written is discharged. In Figure 2.4 it is desired to set $\mathrm{Q}$ to ' 0 ', and hence $\overline{\mathrm{Q}}$ to ' 1 '. Thus, BL is discharged, causing $\mathrm{Q}$ to be discharged through the access transistor M5 when the Word Line (WL) is enabled (Refer to Figure 2.5). The PMOS transistor M2 opposes this operation by sending current into Q. Therefore, the load transistors (M2 and M4) must be weaker than the access transistors (M5 
and M6) so that nodes Q and $\overline{\mathrm{Q}}$ can be pulled low enough to change the state of the cell [18].

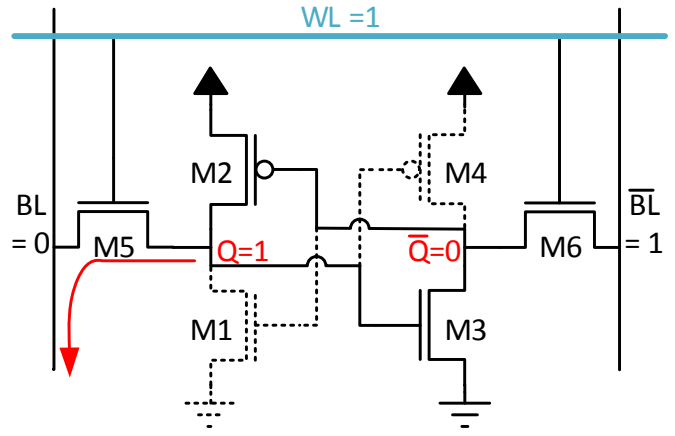

(a) Before Write

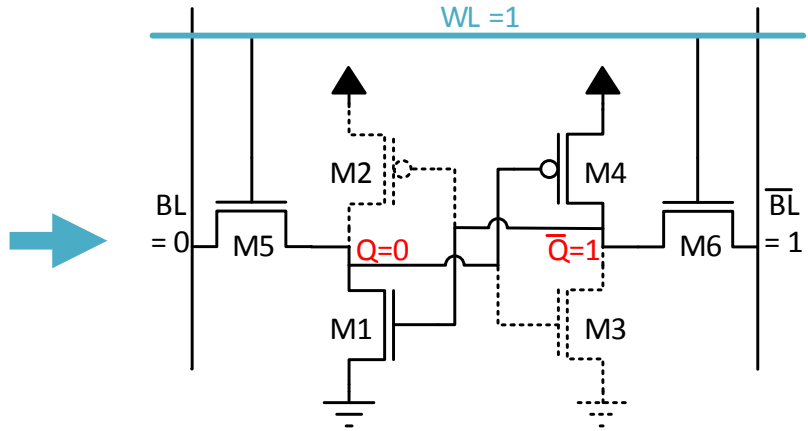

(b) After Write

Figure 2.4: Standard 6T SRAM cell write operation.

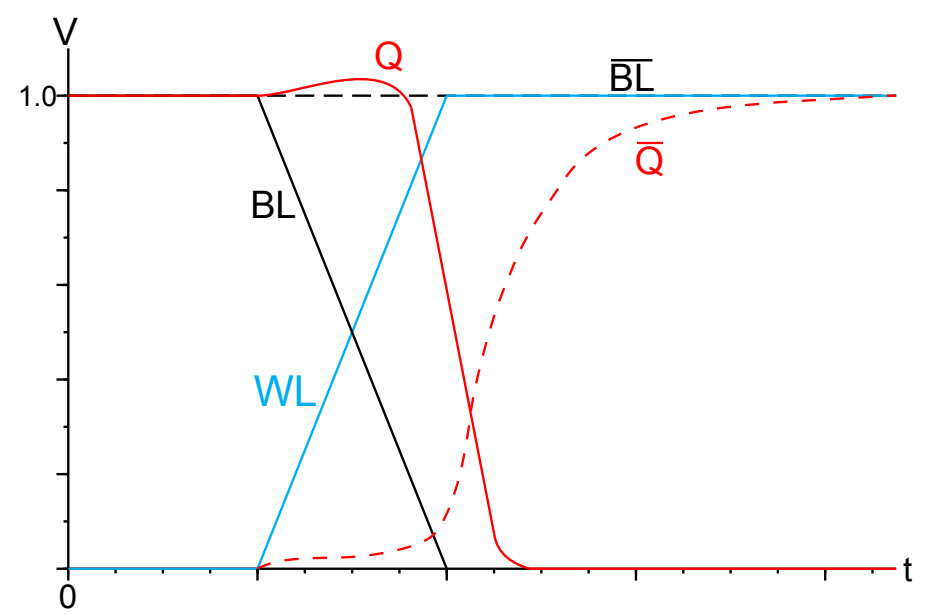

Figure 2.5: Standard 6T SRAM cell write operation timing diagram.

\subsection{Cell stability}

The stability and writiability of an SRAM cell is determined by the hold, read, and write margins. These margins are determined by the static noise margins of the cell in each mode of operation. The hold and read static noise margin (SNM) is a quantification of how much noise can be applied to the inputs of the storage cell 
before a stable state is changed. The write static noise margin is a quantification of how much noise can be tolerated at the inputs of a storage cell before a second stable state is created.

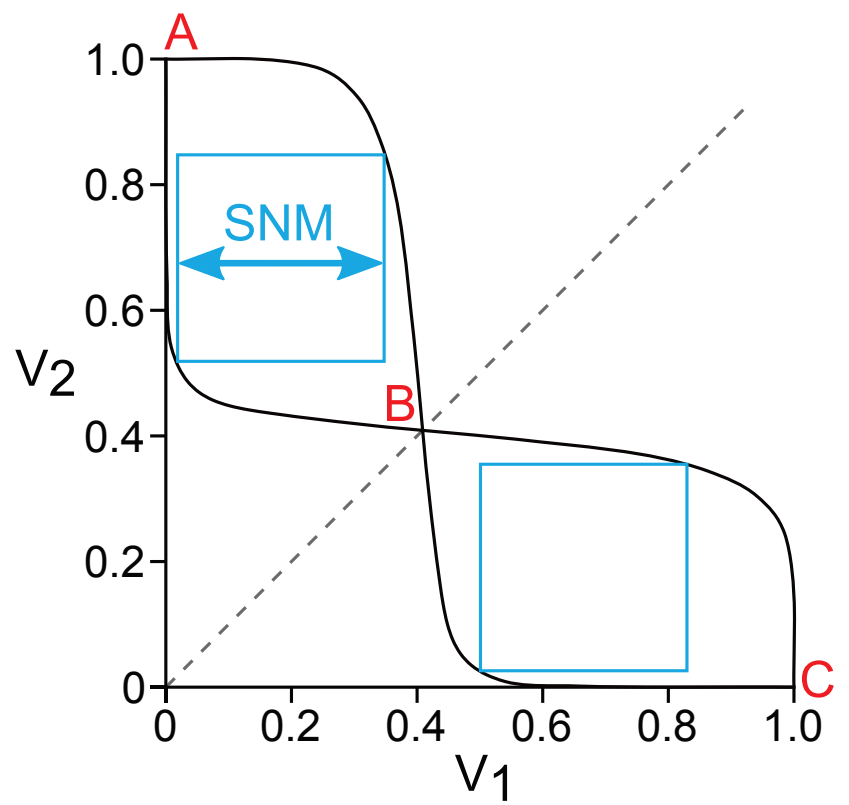

Figure 2.6: Butterfly diagram for the hold static noise margin (HSNM) of a standard $6 \mathrm{~T}$ SRAM cell.

Figure 2.6 is a butterfly diagram for a standard 6T SRAM cell during the hold state. This plot is a two-dimensional state space for a bistable circuit, such as a memory cell. Each point along the curve is a possible combination for the voltages on the bistable nodes $(\mathrm{Q}$ and $\overline{\mathrm{Q}})$. Point $\mathrm{A}$ at the top left of the plot $(0,1)$, where the two curves intersect, marks a stable solution for the system. There is a second stable solution at the bottom right $(1,0)$, marked by point $\mathrm{C}$, where the two curves intersect. In the middle $(0.4,0.4)$, point B marks a metastable solution where the two curves intersect. Any amount of disturbance in the cell at this metastable state will quickly swing the cell to one of the stable states [19].

Figure 2.7(a) shows the best-case, or typical, SNM while in the hold state. The SNM is determined by the width of the largest square that can be placed between the curves. In cases where the butterfly diagram is not symmetric, and the high and low static noise margins are not equal, the SNM is the lesser of the two cases. The largest square shows the maximum amount of noise that can be applied before 


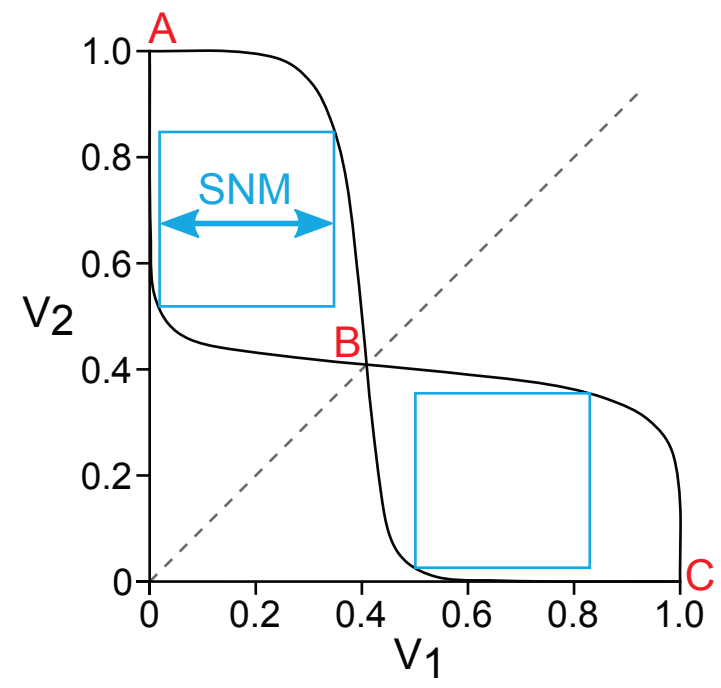

(a) Butterfly diagram for the best-case SNM for a standard 6T SRAM cell.

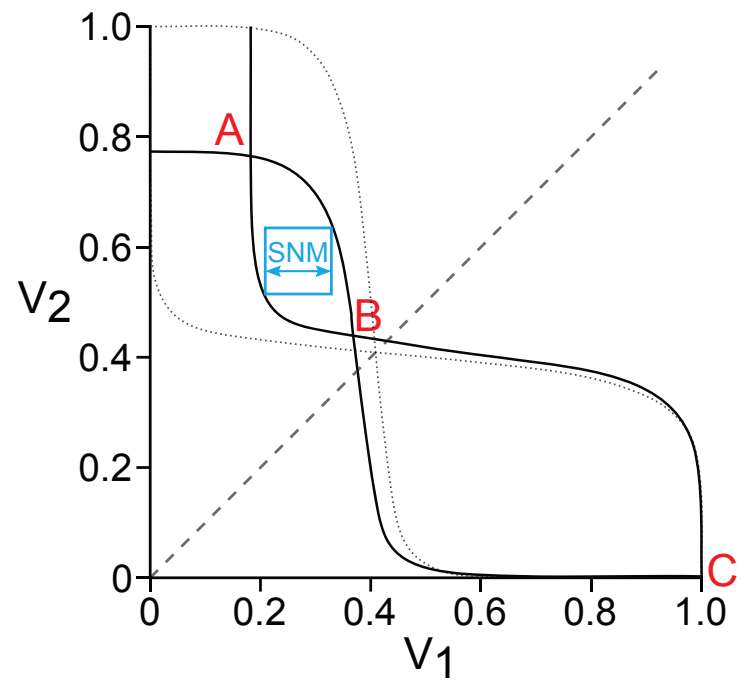

(b) Butterfly diagram for the case where noise is applied into the storage cell for a standard $6 \mathrm{~T}$ SRAM cell. The butterfly diagram for the bestcase SNM is included in the diagram as a dotted line.

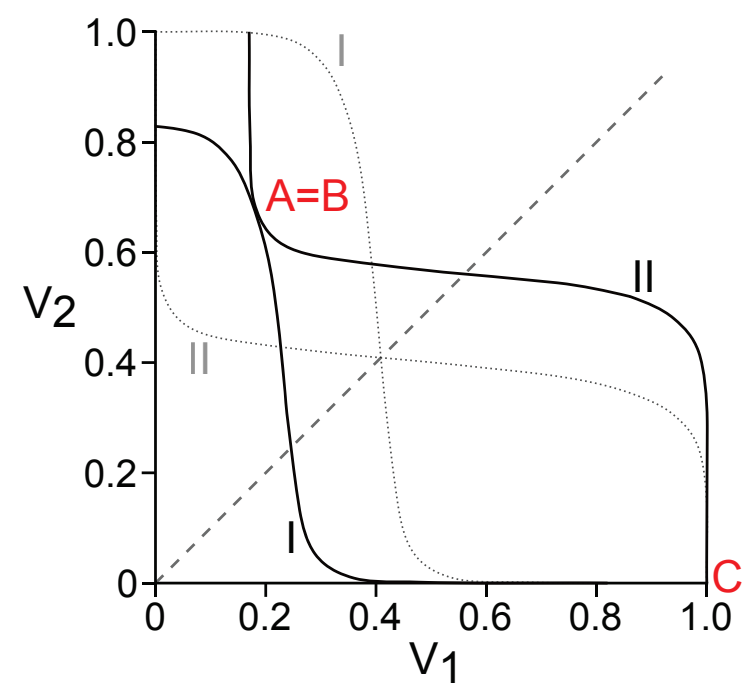

(c) Butterfly diagram for the worst-case noise applied into the storage cell for standard 6T SRAM cell. The butterfly diagram for the best-case SNM is included in the diagram as a dotted line.

Figure 2.7: Butterfly diagrams for 6T SRAM. 
a stable state is lost, assuming equal but opposite noise sources are applied to both sides of the storage cell (refer to Figure 2.8). In the case of Figure 2.7, the worst-case noise is the maximum noise that can be applied until a stable point (point A) and the metastable point (point B) coincide. Any more noise in the system will yield only one intersection point (point $\mathrm{C}$ in this example). Figure 2.7(b) shows noise being applied to the storage cell; Points A and B are closer together, but there are still two stable points and one metastable point. Figure 2.7(c) shows the transfer characteristics when the maximum tolerated noise is applied. Note that the two voltage curves move in opposite directions of equal magnitude. Curve I has had -SNM Volts of noise applied at node V2 and Curve II has had +SNM Volts of noise applied at node V1 [3].

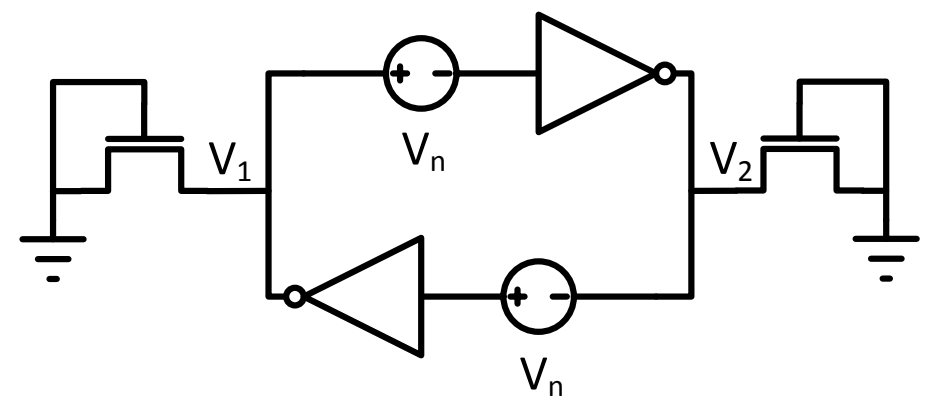

Figure 2.8: Test circuit for measuring the hold static noise margin (HSNM).

The butterfly curve while in the read state is very similar to the hold state. The read SNM determines how much noise can be applied to the storage cell, when reading, before the cell switches its state.

The butterfly curve while in the write state differs from the other two cases, as can be seen in Figure 2.9(a). The write SNM is determined by the width of the smallest square that can be placed between the two curves. When writing, only one stable state is desired to ensure writing the correct value (i.e. Point $\mathrm{A}$ in the figure). The static noise margin in this case determines how much noise can be applied to the storage cell during the write operation before a second state is created (Point B in Figure 2.9(b)). When a second state is created, it is not guaranteed which value will be written to the cell. 


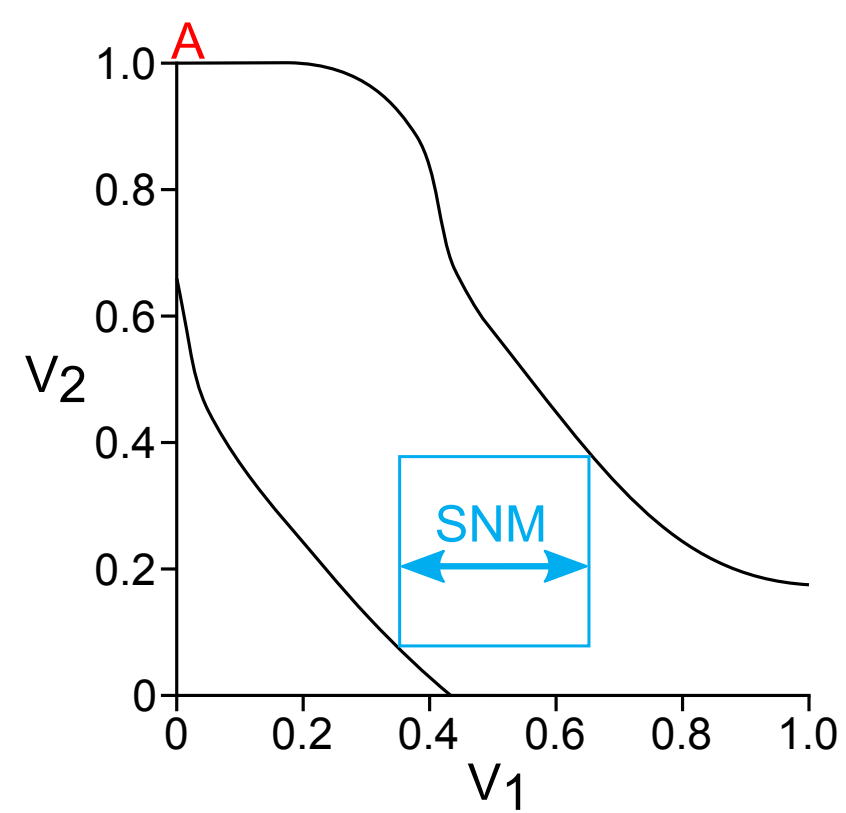

(a) Butterfly diagram for the best-case write SNM for a standard 6T SRAM cell.

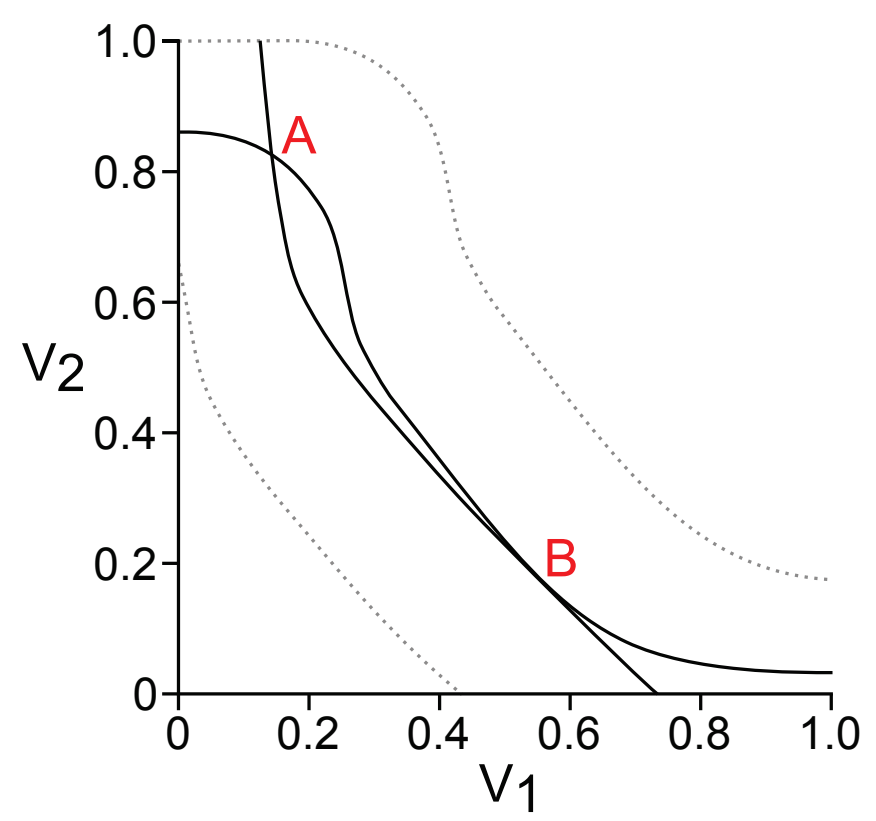

(b) Butterfly diagram for the write mode where noise is applied to the storage cell in a standard 6T SRAM cell. The SNM square from the best-case SNM is included in the diagram for comparison. Curve I has had $+\mathrm{SNM} \mathrm{V}$ of noise applied at node V2 and Curve II has had -SNM V of noise applied at node V1.

Figure 2.9: WSNM butterfly diagrams for 6T SRAM. 


\subsubsection{Measuring SNM}

Figure 2.8 shows the test circuit for measuring the hold static noise margin (HSNM). Noise is applied to the storage cell when the SRAM cell is in the holding state (i.e. the access transistors are all off). In Figure 2.8, $V_{n}$ is the amount of noise voltage applied. The butterfly diagram that determines the SNM is the voltage transfer characteristics of the cross-coupled inverters. That is, the butterfly curve is generated by plotting the input voltage vs the output voltage of each inverter. The width of the largest square that can be placed between the two curves is the HSNM (see Figure 2.6).

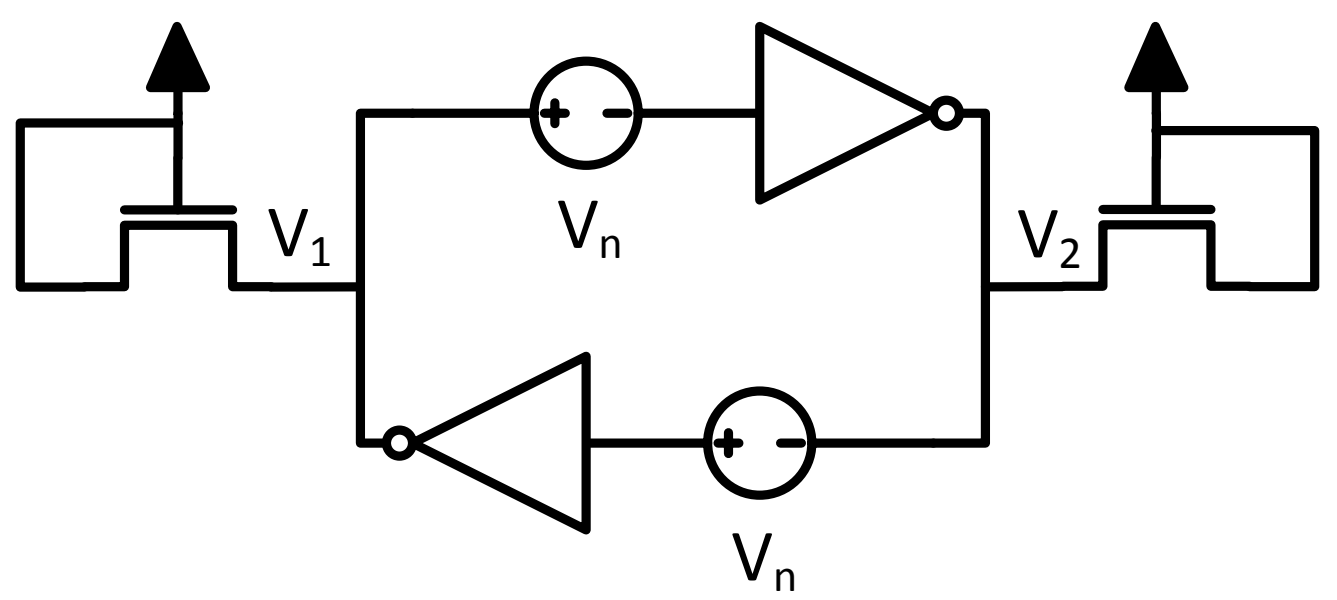

Figure 2.10: Test circuit for measuring the read static noise margin (RSNM).

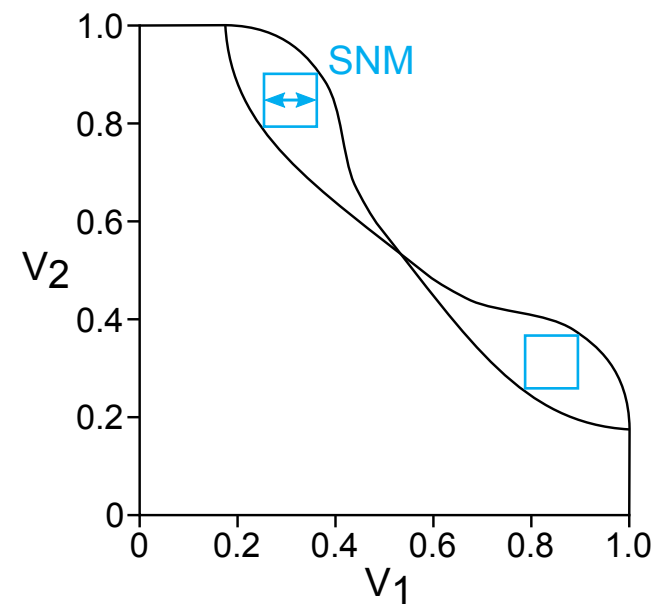

Figure 2.11: Butterfly diagram for the read static noise margin (RSNM) for a standard 6T SRAM cell [2]. 
The test circuit for determining the read static noise margin (RSNM) is depicted in Figure 2.10. Measuring the RSNM of the cell is done the same way as in the hold state, but with the SRAM cell in the read state. That is, by applying noise to the storage cell when the access transistors are on and the bit-lines are high. The access transistors end up pulling the node voltages up, distorting the voltage transfer characteristics and resulting in a lower SNM than in the hold state, as shown in Figure 2.11. The RSNM can be improved by decreasing the strength of the access transistors, and increasing the strength of the drive transistors. Access transistors can be weakened by making them smaller relative to the drive transistors, and/or by reducing the Word Line voltage relative to $V_{D D}$. The drive transistor can be made stronger by making them larger relative to the access transistors, increasing $V_{D D}$, and/or increasing $V_{t n}$.

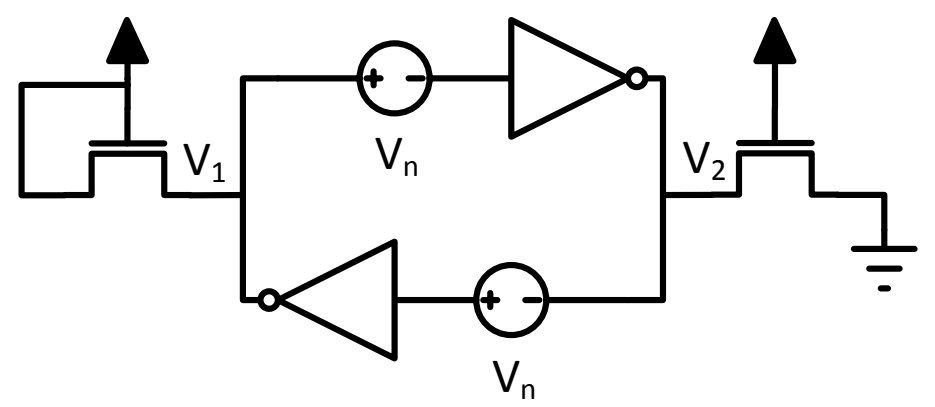

Figure 2.12: Test circuit for measuring the write static noise margin (WSNM).

Obtaining the write static noise margin (WSNM) is done similarly to the previous two cases. The test circuit can be seen in Figure 2.12. To write to the SRAM cell, both access transistors are enabled while one bit-line is pre-charged high and the other is discharged to ' 0 '. The smallest square that can be placed between the curves determines the WSNM. Conversely to improving the RSNM, the WSNM improves by increasing the strength of the access transistors, and by decreasing the strength of the drive transistors. The access transistors are strengthened by making them larger relative to the drive transistors, and/or by increasing the Word Line voltage. The drive transistors can be made weaker by making them smaller relative to the access transistors.

It's important to note that there is a vast distribution of read, write, and hold static noise margins on each chip due to threshold voltage mismatches caused by random dopant fluctuations in the many cells. This is especially a problem in nanometre 
processes [19]. Therefore, to obtain a good estimation of static noise margins, many simulations must be run in the presence of random process variations.

\subsubsection{Measuring SNM by Circuit Simulation}

Obtaining the SNM by drawing the largest square is a good graphical estimation, but a method to calculate the SNM is needed for running exhaustive simulations (i.e. Monte Carlo Simulations). Seevinck et al. created a method to do this [3].

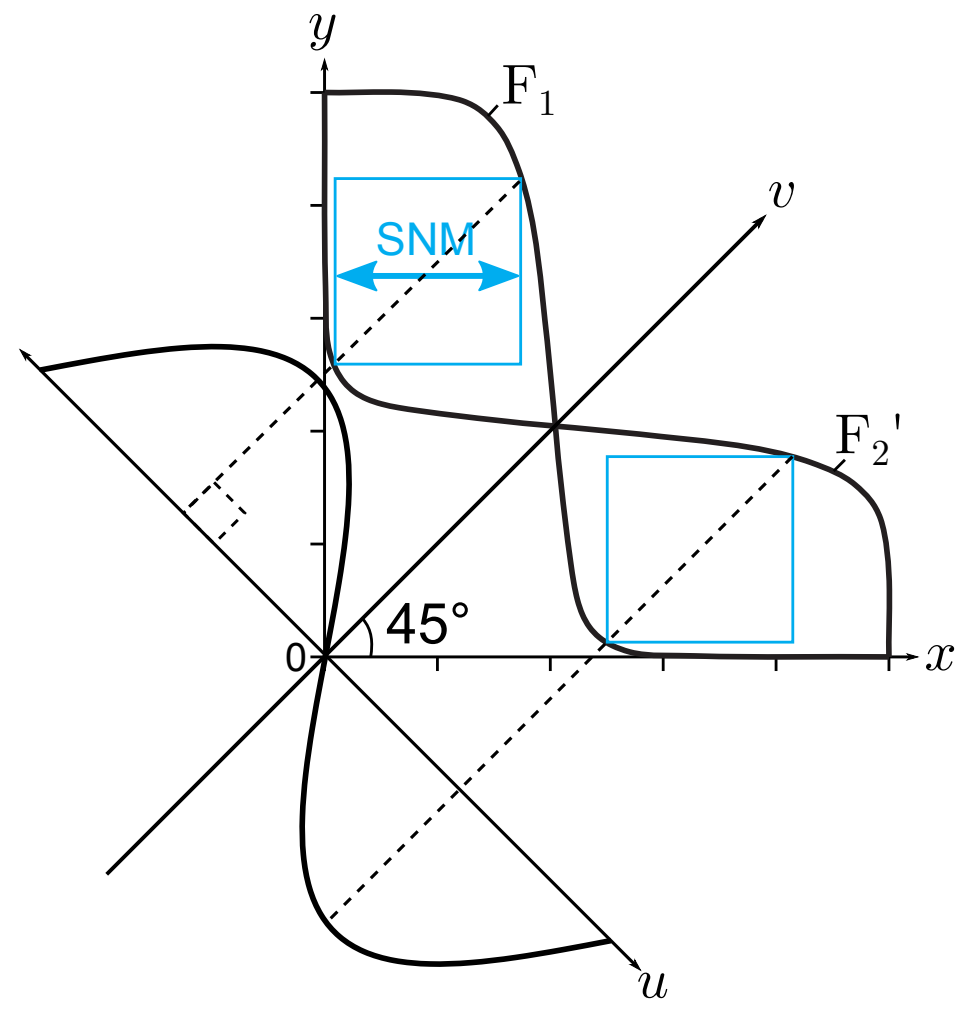

Figure 2.13: Estimation of SNM in a $45^{\circ}$ rotated coordinate system [3].

The method involves rotating the axis $45^{\circ}$ and plotting the difference between the two curves (see Figure 2.13). This difference is the distance between the two curves. Thus, the maximum absolute difference (multiplied by $\cos 45^{\circ}$ ) is the SNM.

By assuming that the voltage transfer characteristic of one inverter is $\mathrm{y}=\mathrm{F}_{1}(\mathrm{x})$, and the other is $\mathrm{y}=\mathrm{F}_{2}^{\prime}(\mathrm{x})$, which is the mirrored version of $\mathrm{y}=\mathrm{F}_{2}(\mathrm{x})$, the curves can be 
transferred to the $45^{\circ}$ rotated coordinate system $(\mathrm{u}, \mathrm{v})$ by using the following equations

$$
\begin{aligned}
& x=\frac{1}{\sqrt{2}} u+\frac{1}{\sqrt{2}} v, \\
& y=-\frac{1}{\sqrt{2}} u+\frac{1}{\sqrt{2}} v .
\end{aligned}
$$

Substituting $\mathrm{y}=\mathrm{F}_{1}(\mathrm{x})$ into (2.1) gives

$$
v=u+\sqrt{2} F_{1} \frac{1}{\sqrt{2}} u+\frac{1}{\sqrt{2}} v
$$

Since $\mathrm{F}_{2}^{\prime}(\mathrm{x})$ is the mirrored version of $\mathrm{F}_{2}(\mathrm{x})$, to obtain it's transformation, $\mathrm{F}_{2}(\mathrm{x})$ is first mirrored with respect to the v-axis and then transformed to the $(\mathrm{u}, \mathrm{v})$ coordinate system. This transformation results in the equations

$$
\begin{aligned}
& x=-\frac{1}{\sqrt{2}} u+\frac{1}{\sqrt{2}} v, \\
& y=\frac{1}{\sqrt{2}} u+\frac{1}{\sqrt{2}} v .
\end{aligned}
$$

Substituting $\mathrm{y}=\mathrm{F}_{2}(\mathrm{x})$ into (2.3) gives

$$
v=-u+\sqrt{2} F_{1}-\frac{1}{\sqrt{2}} u+\frac{1}{\sqrt{2}} v
$$

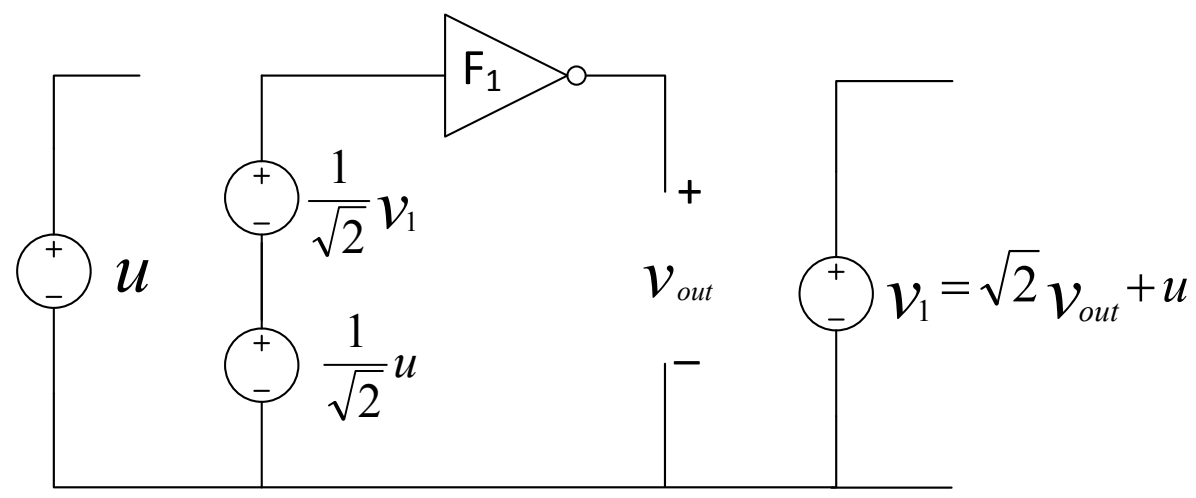

Figure 2.14: Circuit implementation of (2.2).

Equations (2.2) and (2.4) are mathematical representations of the inverters in an SRAM cell. Translating these equations into circuits results in Figure 2.14 and 


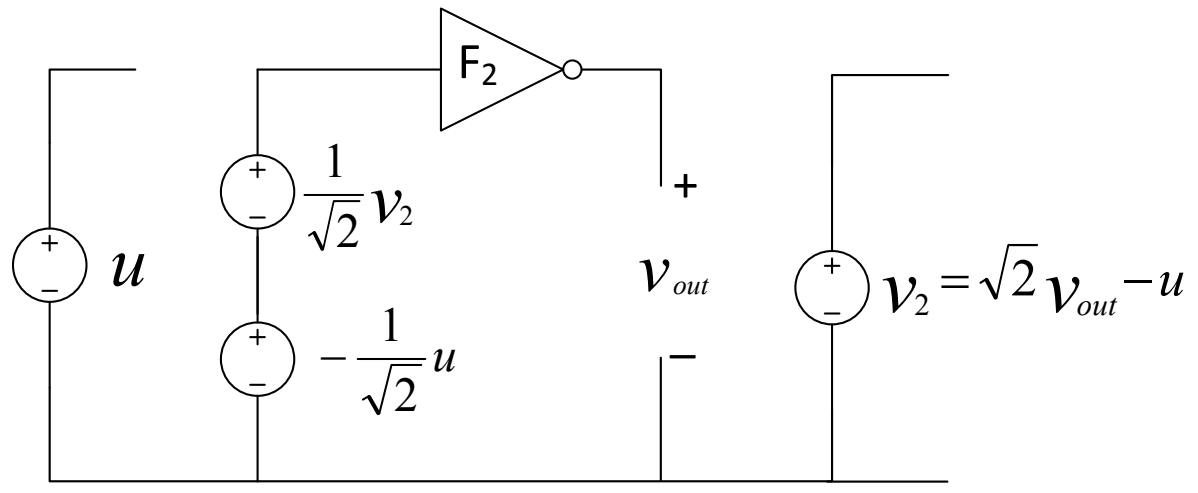

Figure 2.15: Circuit implementation of (2.4).

Figure 2.15, respectively. By setting the Word-Lines and Bit-Lines to their respective values and sweeping $u$ from $-\mathrm{V}_{D D} / \sqrt{2}$ to $\mathrm{V}_{D D} / \sqrt{2}$ the SNM of each operation can be obtained.

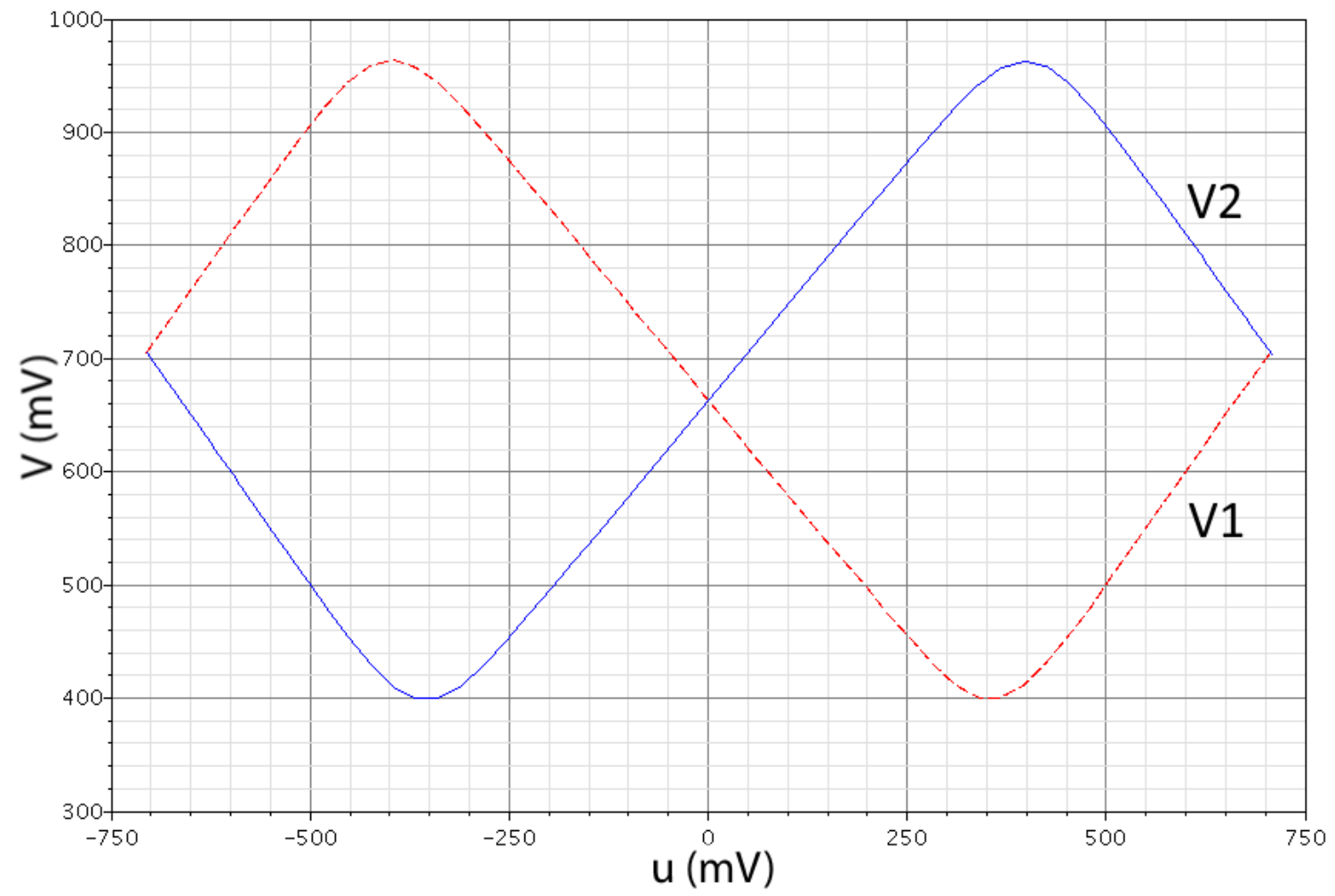

Figure 2.16: Butterfly (SNM) curves for the hold operation transposed onto the $45^{\circ}$ rotated coordinate system $(\mathrm{u}, \mathrm{v})$. The dashed curve is $\mathrm{v}_{1}$ and the solid curve is $\mathrm{v}_{2} . \mathrm{V}_{D D}$ is $1.0 \mathrm{~V}$ in this case, so $u$ was swept from $-707 \mathrm{mV}$ to $707 \mathrm{mV}$. 


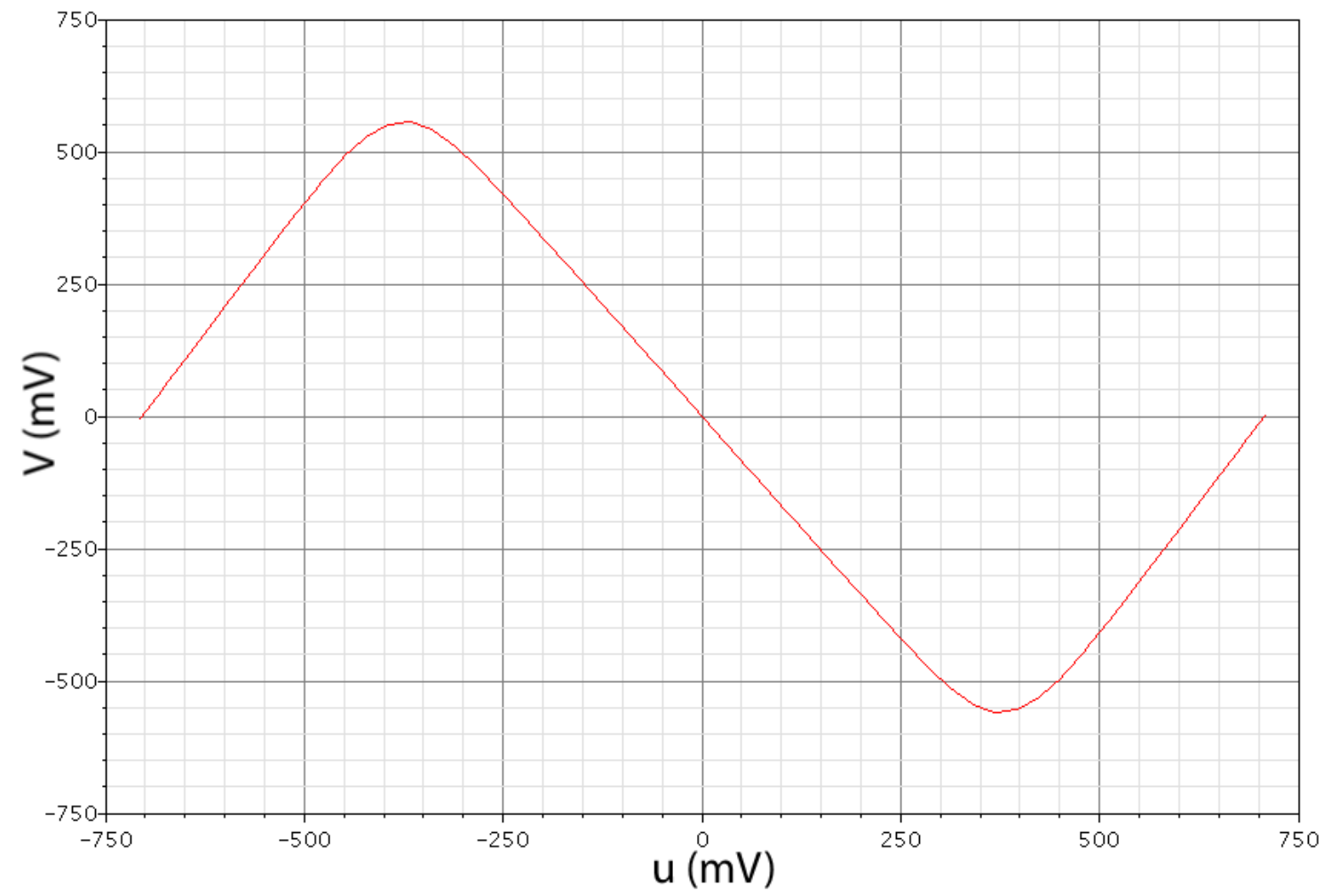

Figure 2.17: The difference between the two butterfly (SNM) curves $\left(\mathrm{v}_{1}-\mathrm{v}_{2}\right)$ in Figure 2.16.

Figure 2.16 shows the result of simulating the circuits in Figures 2.14 and 2.15 in the hold operation. The difference between the two solutions $\left(\mathrm{v}_{1}-\mathrm{v}_{2}\right)$ can be seen in Figure 2.17. The absolute values of the maximum and minimum are the diagonal lengths of the largest squares. Therefore, dividing the smaller value of the two by $\sqrt{2}$ gives the SNM of the cell [3].

\subsection{Low-power SRAM}

All SRAM cells have a minimum voltage at which they can reliably operate, since the SNM depends on $V_{D D}$. Figure 2.18 shows how the read SNM diminishes for a standard 6T SRAM cell as $V_{D D}$ is decreased. Moreover, as the operating voltage decreases, process, voltage, and temperature variations become more apparent; This results in a greater variation in the SNM. When 6T SRAM cells are employed the minimum $V_{D D}$ is typically between $0.7-1.0 \mathrm{~V}$ mainly due to the diminishing RSNM. 
The minimum voltage of operation is around $0.7 \mathrm{~V}$ in a $90 \mathrm{~nm}$ process. As such, $6 \mathrm{~T}$ SRAM cells are not suitable for sub-threshold operation [20].

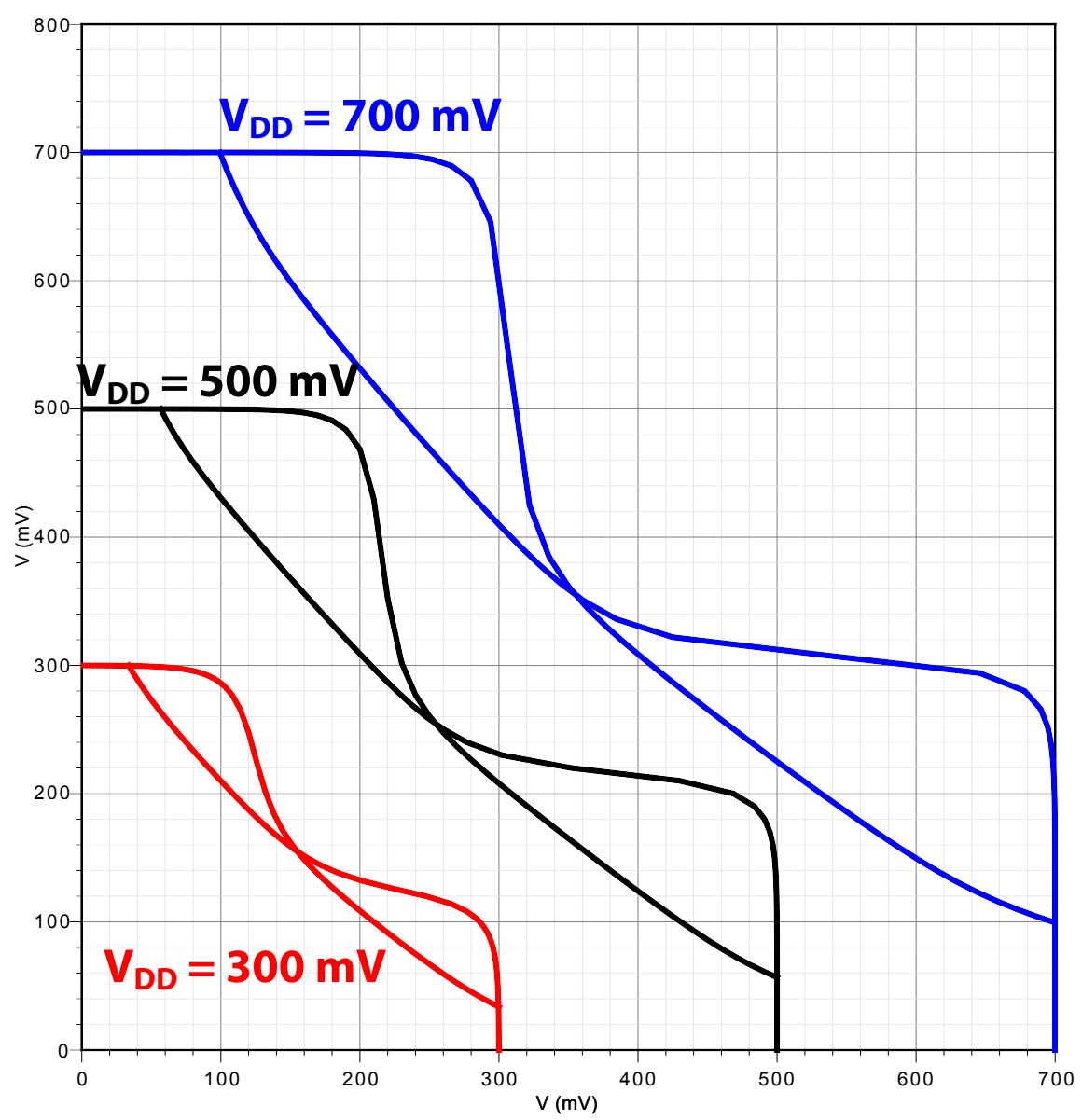

Figure 2.18: Read SNM comparision for $6 \mathrm{~T}$ SRAM cell at different $V_{D D}$.

A few techniques have been proposed to increase the read SNM of the standard 6T SRAM cell to allow for lower voltage operation. Some examples include lowering the word-line voltage in respect to $\mathrm{V}_{D D}$ [21], dynamically increasing cell $\mathrm{V}_{D D}$ during reads [22], utilizing a negative GND [23], and pulsing the word-line or bit-line (that takes advantage of the larger dynamic noise margin) [24]. However, these methods require additional circuitry and, generally, consume more energy. Lowering the wordline voltage increases the read SNM, but decreases the read speed.

Various techniques have also been proposed to increase the write SNM of SRAM cells, but, as mentioned, the RSNM is predominantly the bottleneck when lowering $V_{D D}$. Dynamically lowering the cell $\mathrm{V}_{D D}$ during writes [22], floating the cell ground 
during writes [25], increasing the word-line voltage [12], and keeping a negative voltage on the bit-line [13] all improve the reliability when writing to the cell. These techniques all require additional circuitry, increase power consumption, and, in some cases, decrease the hold SNM of neighbouring cells.

While these assistance methods can help the standard 6T SRAM operate at a lower voltage, it still does not make it suitable for sub-threshold operation. An arguably better solution is to improve the SRAM cell.

The second most common SRAM cell, and the standard choice when operating at voltages below those supported by the standard 6T SRAM cell, is the traditional $8 \mathrm{~T}$ SRAM cell in Figure 2.19. The 8T SRAM cell addresses the read SNM problem by isolating the read bit-line from the storage cell at the cost of around $30 \%$ increase in area [26].

The write operation of the $8 \mathrm{~T}$ SRAM cell is the same as in the conventional $6 \mathrm{~T}$ cell, but the bit-lines and word-lines used during a write are only used for the write operation. Exactly like in the 6T SRAM cell, the write bit-lines (WBL and $\overline{\mathrm{WBL}}$ ) are precharged high before the operation begins. When the write operation starts, the write bit-line connected to the node where ' 0 ' is desired to be written is discharged. When the write word line (WWL) is enabled, one side of the storage cell is selectively discharged through the discharged bit-line [26].

The read operation for the 8T SRAM cell has it's own read word-line, a separate read bit-line, and, as a result, each cell has an additional access transistor (M8 in Figure 2.19) exclusively for reads. Before the read operation occurs, the read bit-line (RBL) is precharged high. To begin the read operation, the read word line (RWL) is raised high, enabling the read access transistor (M8). Depending on the value stored in the cell, the read decoupling transistor (M7) is selectively enabled. If the read decoupling transistor is enabled, the read bit-line is discharged through the read access and decoupling transistors. Thus, it will be read as a logic ' 0 ' by the peripheral circuitry. If the read decoupling transistor is disabled, the read bit-line remains high and will be read as a logic ' 1 ' by the peripheral circuitry [26].

Another key issue for sub-threshold SRAM is the relatively poor ratio of $\mathrm{I}_{O N}$ to $\mathrm{I}_{O F F}$ of the transistors. This poor ratio puts a limit on the number of cells that can be connected to a bit-line. With many cells connected to a bit-line, the total leakage current in a bit-line can exceed the $\mathrm{I}_{O N}$ current of a SRAM cell during reads. To overcome this problem without adding more transistors to limit leakage, most 


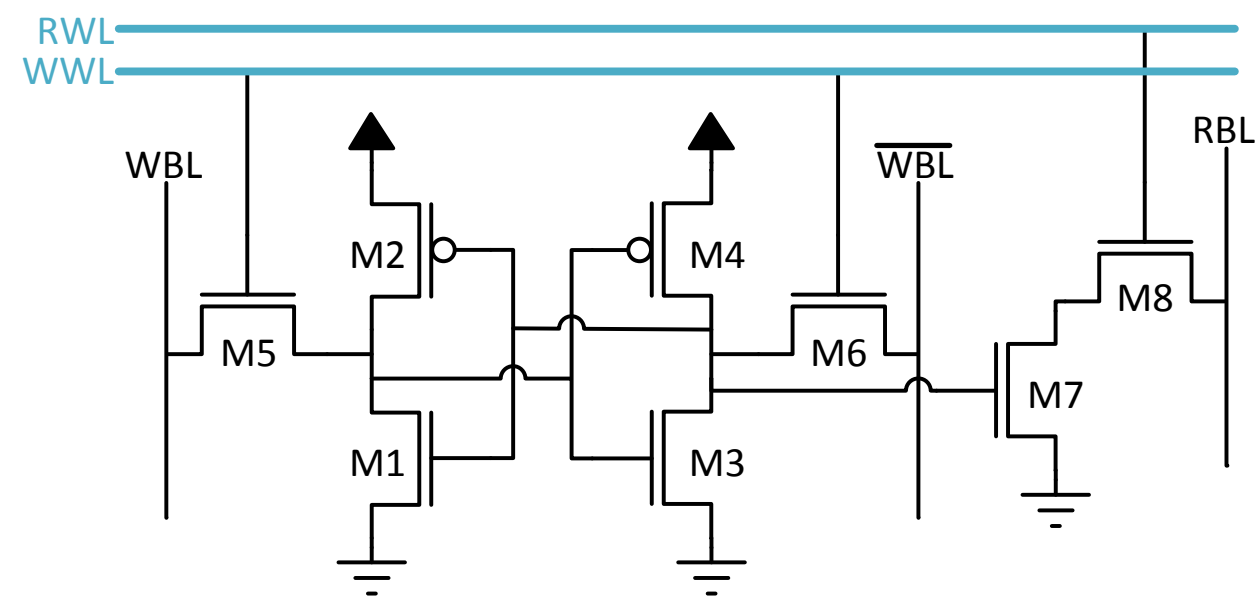

Figure 2.19: The traditional 8T SRAM cell.

architectures implement hierarchical bit-lines.

\subsection{Hierarchical bit-lines}

The classical SRAM architecture is illustrated in Figure 2.20. The classical structure consists of an array of memory cells with $2^{m}$ rows and $2^{n}$ columns. Each SRAM cell in the same column share the same bit-lines.

When utilizing conventional 6T SRAM cells, the bit-line capacitance mostly consists of the diffusion junction capacitance of the access transistor connected to the bit-line and metal capacitance of the bit-line. Thus, the bit-line capacitance can be expressed as [27]

$$
C_{B L}=C_{J u n c t i o n A c c e s s T r a n s i s t o r} \times N_{\text {CellsConnectedToBit-line }}+C_{\text {Metal }} .
$$

It is easy to see that reducing the number of cells on the bit-line can have a significant effect on the bit-line capacitance. The bit-line capacitance has a major effect on the $\mathrm{RC}$ delay and grows proportionally with each cell added to the bit-line. The bit-line capacitance also affects the power dissipation $(\mathrm{P})$ linearly since

$$
P=C V_{D D} V_{s w} f
$$

where $f$ is the switching frequency, and $V_{s w}$ is the voltage swing of the bit-line. 


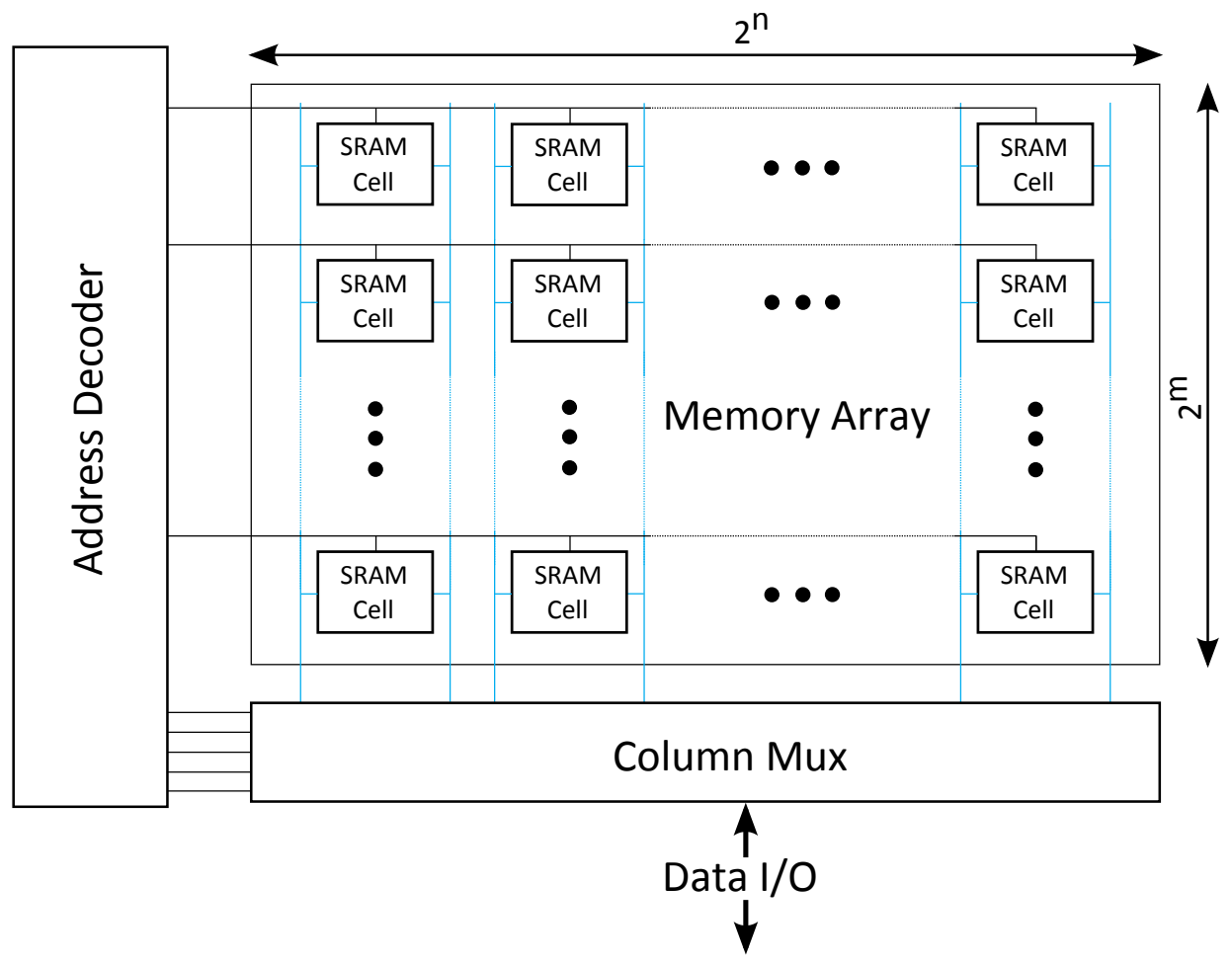

Figure 2.20: Classical SRAM array architecture [4].

A modified SRAM architecture is known as hierarchical or divided bit-lines. Hierarchical bit-lines subdivide the single large array into several smaller sub-arrays, reducing the number of SRAM cells attached to a bit-line. Each smaller sub-array is connected to a global bit-line and presents only a small load relative to all the cells in a single row of the classic architecture. This decreases the RC delay and power dissipation. Additionally, it is possible to only enable the required bit-lines in a subarray, which can further reduce the overall power dissipation. Figure 2.21 shows an example of an hierarchical or divided bit-line architecture. In this architecture, global bit-lines run along the SRAM array columns and several sub-bit-lines connect a small group (4 SRAM cells in this case) of SRAM cells vertically. These sub-bit-lines are connected to the global bit-lines through pass transistors, reducing the number of transistors directly connected to the global bit-line.

Hierarchical bit-lines also help alleviate the problem of bit-line leakage by limiting the number of SRAM cells on each bit-line, without limiting the total number of cells in an SRAM block. There is a small area penalty that is incurred by utilizing 


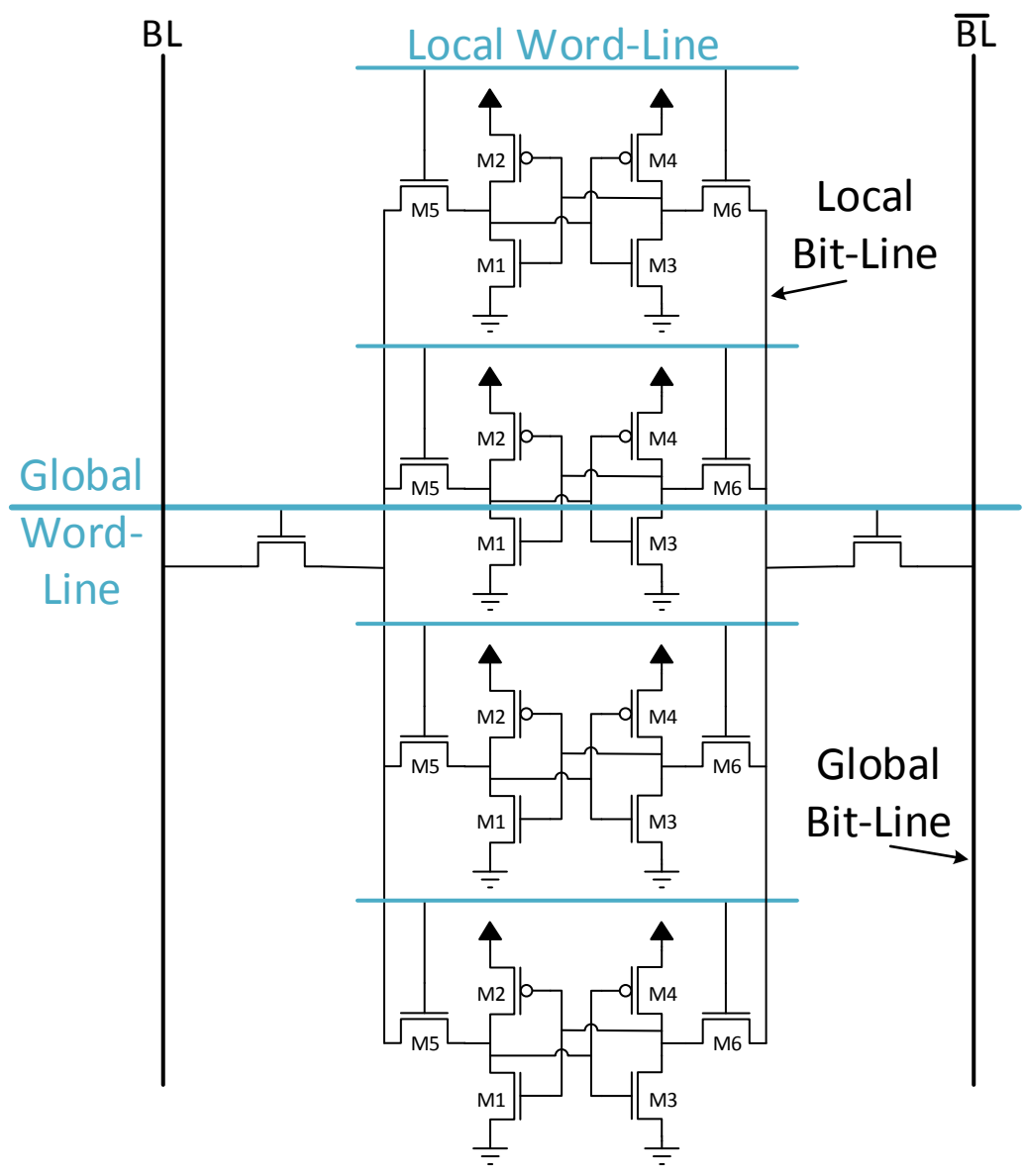

Figure 2.21: Hierarchical or divided bit-line architecture [4].

hierarchical bit-lines, but it can help to allow for sub-threshold operation (by reducing disturbances and the capacitance on a bit-line) and reduces power dissipation [4].

\subsection{Single-ended SRAM}

Using single-ended SRAM cells is a fairly common approach for reducing leakage current and power consumption. Traditional SRAM architectures contain multiple bit-lines with large capacitances in every column. Due to these large capacitances, charging and discharging the bit-lines results in a relatively significant power consumption. A single-ended SRAM architecture reduces the number of bit-lines to one per column, decreasing the active power consumption by at least half. Moreover, since there is only one bit-line from which leakage current can flow into a cell, there is also a significant reduction in the static power consumption. Figure 2.22 displays the 
simplest single-ended SRAM cell architecture. It is basically the standard 6T SRAM cell with one access transistor and bit-line removed.

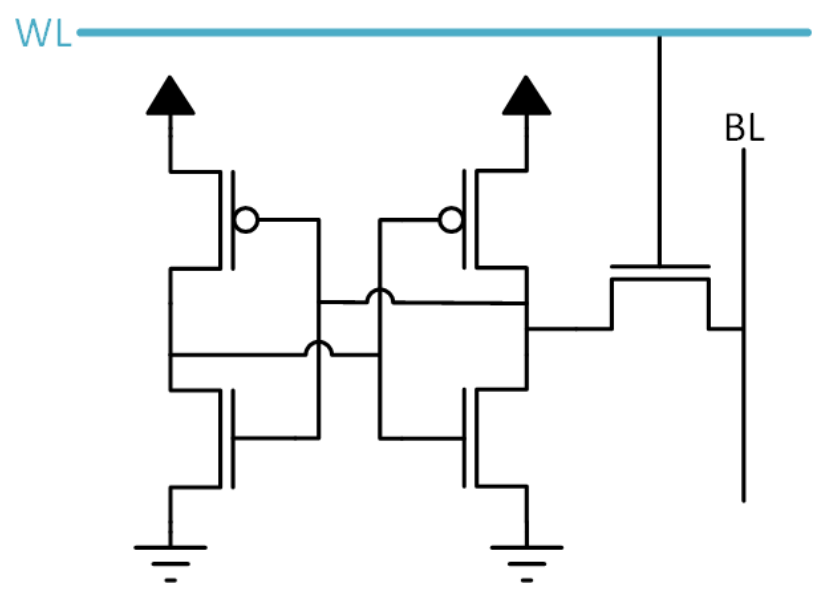

Figure 2.22: Simplest single-ended SRAM cell architecture [5].

Single-ended SRAM has it's follies however. Typically, a single ended approach utilizes the same bit-line and a single access transistor to write a ' 0 ' and a ' 1 ' into the same node of the storage cell. As discussed previously, writing a ' 0 ' into one side of the cross-coupled inverters is not an issue because of the strong NMOS drive (or pull-down) and access transistors. However, writing a ' 1 ' requires the ability to overcome the strong NMOS drive transistors. A strong ' 1 ' writability requires the use of write assist circuitry, such as weakening one side of the cross-coupled inverters by floating its ground, that comes at the cost of increased area and power consumption. Moreover, write assist circuitry generally has the unintended consequence of lowering the hold static noise margin of half-selected cells [21].

A single-ended cell architecture similar to that in Figure 2.22, where there is a single access transistor connected to a large capacitance bit-line, has a read SNM similar to that in the standard 6T case. As such, it is unsuitable for low-power operation. Many single-ended SRAM bit-cell architectures, however, provide separate read and write paths while utilizing a single bit-line. This allows for optimized paths for each operation to increase the static noise margins for each individual operation. Figure 2.23 shows an example of a single-ended SRAM bit-cell with different read and write paths. 


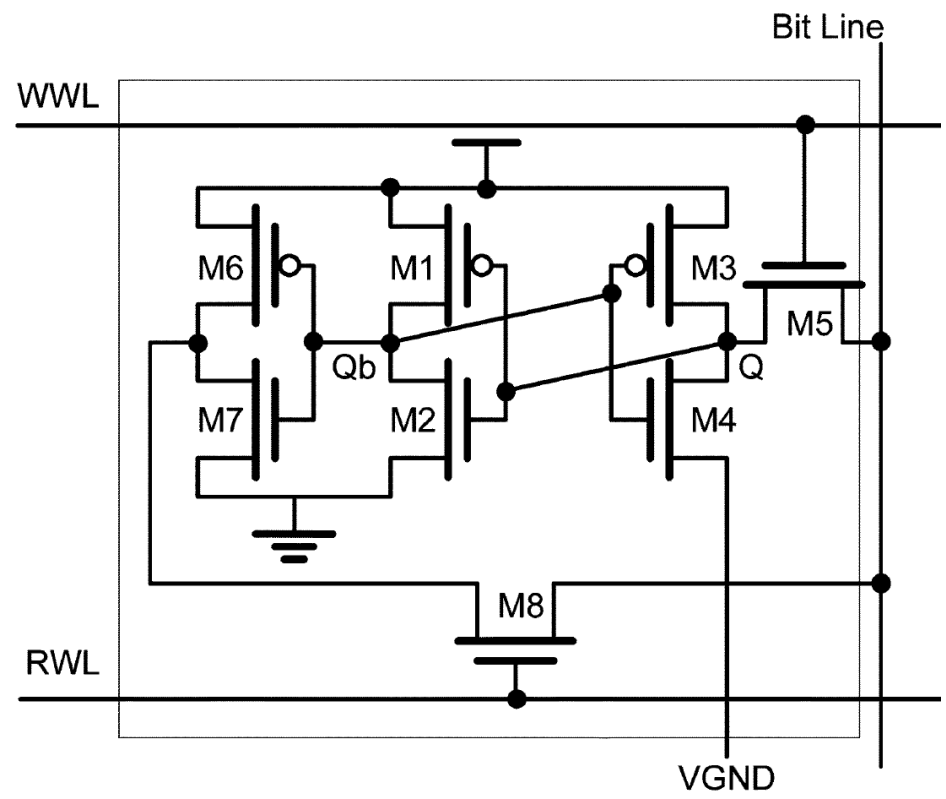

Figure 2.23: Single-ended SRAM bit-cell with different read and write paths [6]. (C)2010 IEEE

\subsection{Half-Select disturb}

An issue that becomes more apparent in low-voltage and sub-threshold SRAM architectures is half-select disturb. Half-select disturb occurs in a cell when the word-line for the row is selected, but the column is unselected, leaving the bit-line floating high. This is perhaps better illustrated by Figure 2.24. The disturbed cells in the disturbed columns have their access transistors enabled for the write operation and their bitlines are left floating high. Therefore, it is possible for noise in the bit-lines to flip the stored data in the disturbed cells. This presents a compromise between write ability and the disturb margin. The current flowing through the access transistors needs to be large enough to flip the stored data in the storage cell, but not too large that the stored data flips when the cell is disturbed [7].

\subsection{Bit-interleaving}

As SRAM cells enter sub-threshold operation, there is an increase in soft-error rates. Soft errors are incorrect signals or data that result from the radiation of energetic particles, thermal neutrons, and random noise. Soft errors are often undetectable. 


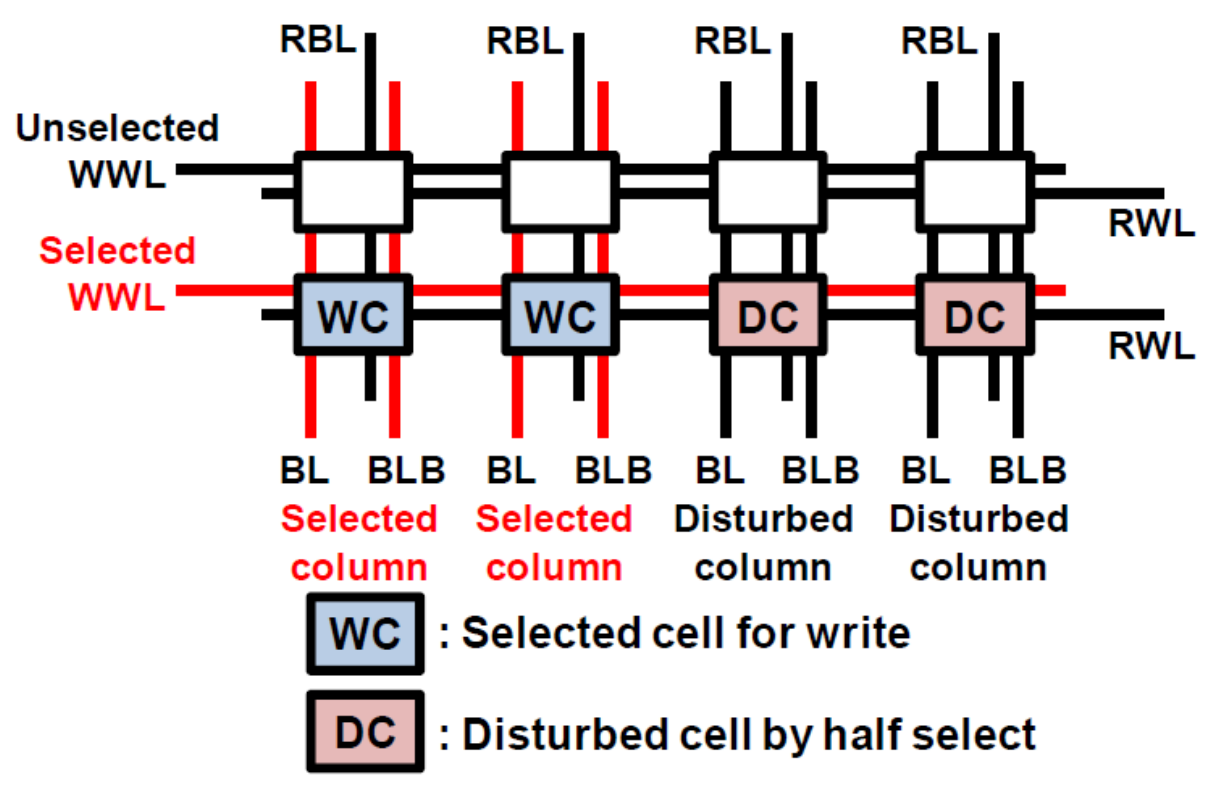

Figure 2.24: Half select disturb problem in a standard 8T SRAM cell array [7]. (C) 2010 IEEE

Generally, when soft errors occur, adjacent bits are also affected [28]. When only one bit in a word is affected, Error-correcting codes (ECC) can recover the affected bit. However, it is difficult to implement on-chip ECC that can recover multiple-bits.

Typically, all bits in a word are stored adjacent to each other in one contiguous row. This makes multiple bits in a word targets for unrecoverable soft errors. However, bitinterleaving can be implemented to separate the bits in a word, so when soft errors do occur, only one bit in a word is affected. As its name implies, this technique interleaves the bits of different words. Figure 2.25 shows an example of bit-interleaving.

In the figure, each bit in the word is far away from each other. Thus, if for example, the first 4 bits in the row are affected by a soft error, only the first bits of the first 4 words are affected, and can be recovered by ECC [8].

\subsection{Physical Design}

As discussed in the introduction, SRAM cells often dominate the area of modern chips. Hence, it is desirable to minimize the area of each cell to improve integration density.

The ideal layout of an SRAM cell is a square to balance the capacitive load on 


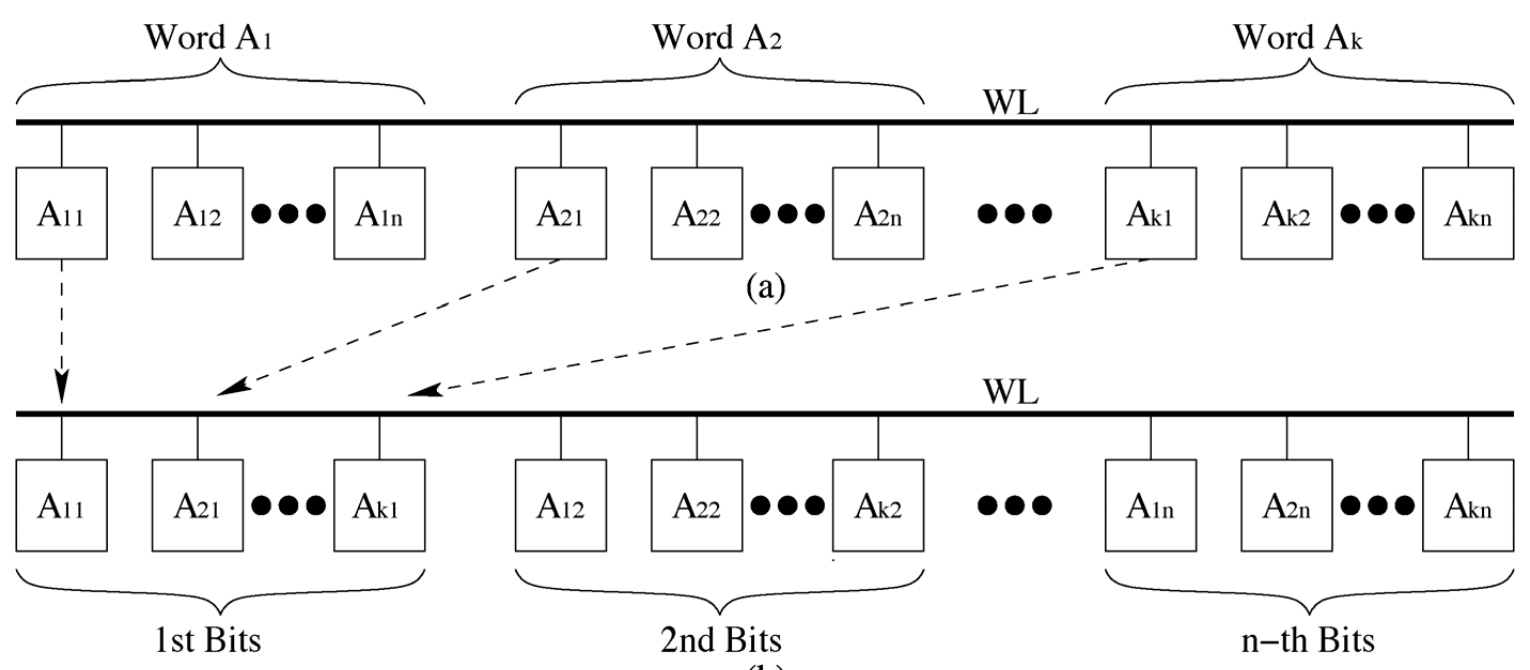

(b)

Figure 2.25: SRAM word organization: the top row shows the typical case where all bits in a word are stored adjacently, unlike the bottom row that shows an implementation of bit-interleaving [8]. (C)2011 IEEE

row and column lines, and balance the area overhead for the decoder and sense/drive circuits. Up until the $90 \mathrm{~nm}$ process generation, a layout similar to that seen in Figure 2.26 was used. This layout is one of the smallest possible layouts for the $6 \mathrm{~T}$ cell. To minimize the area, cells are designed to be mirrored and overlapped to share $V_{D D}$, GND, word-lines, and bit-lines with adjacent cells, as shown in Figure 2.27.

Once the feature size gets below $90 \mathrm{~nm}$, the bends in polysilicon and diffusions in the layout are challenging to fabricate. There is also an increase in process variations due to mask misalignments. To get around these issues, processes under $90 \mathrm{~nm}$ use the thin cell $6 \mathrm{~T}$ cell layout shown in Figure 2.28. In this layout, all transistors are aligned in the same direction; all diffusions run in the vertical direction and all polysilicon in the horizontal direction. Since the cell is long and skinny, the bit-line capacitances, which run vertically, are reduced. The word-lines are longer as a result, but their capacitances are less critical.

An overly long or thin SRAM blcok results in excessive routing area, signal delay, and capacitance. Ideally, the entire SRAM circuit should have a shape close to a square. However, the aspect ratio of the cells, and number of rows and columns can make this difficult. Often, to address the non-square shape of the cells, SRAM 


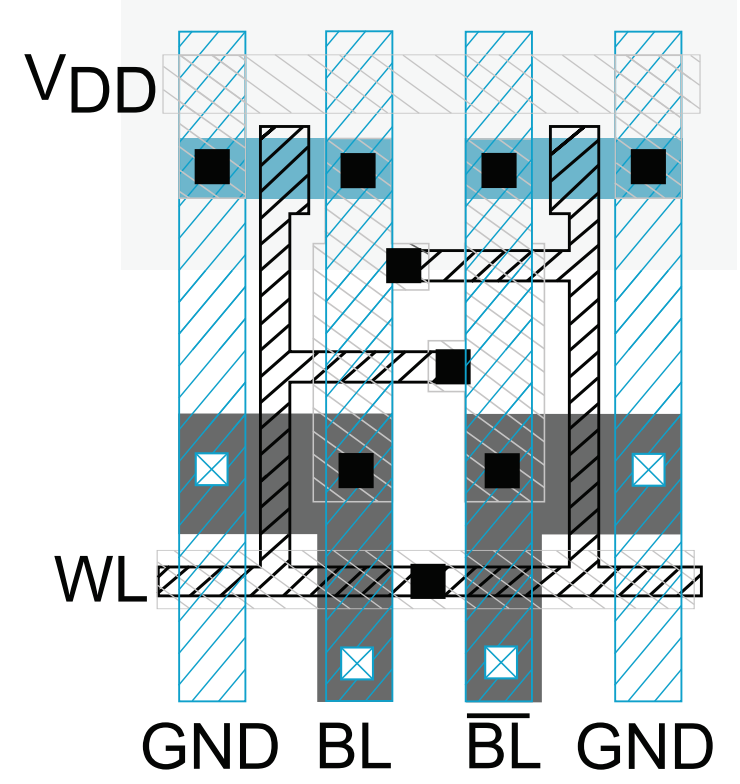

Figure 2.26: Historical layout of 6T SRAM cell [2].

blocks at these feature sizes will consist of more rows than columns to obtain a more square-like shape. This balances the capacitive loads and obtains a more efficient area layout [29].

The width of the drive (or pull-down) transistors shown in Figure 2.28 are larger than that of the load (or pull-up) transistors and access transistors. While this increases the read static noise margin, it also results in a diffusion notch between the access and drive transistors that tends to round out due to lithography limitations. This is illustrated in Figure 2.29. The rounding of the notch may change the effective width of the access transistor and increase variations in the static noise margin. Diffusion-notch-free (or rectangular-diffusion) cells have been proposed in order to mitigate this manufacturing defect. The layout of such cells is illustrated in Figure 2.30. In this layout, all six transistors in the cell have the same minimum widths and lengths. This reduces the read static noise margin along with the process variations in the cell [9]. 


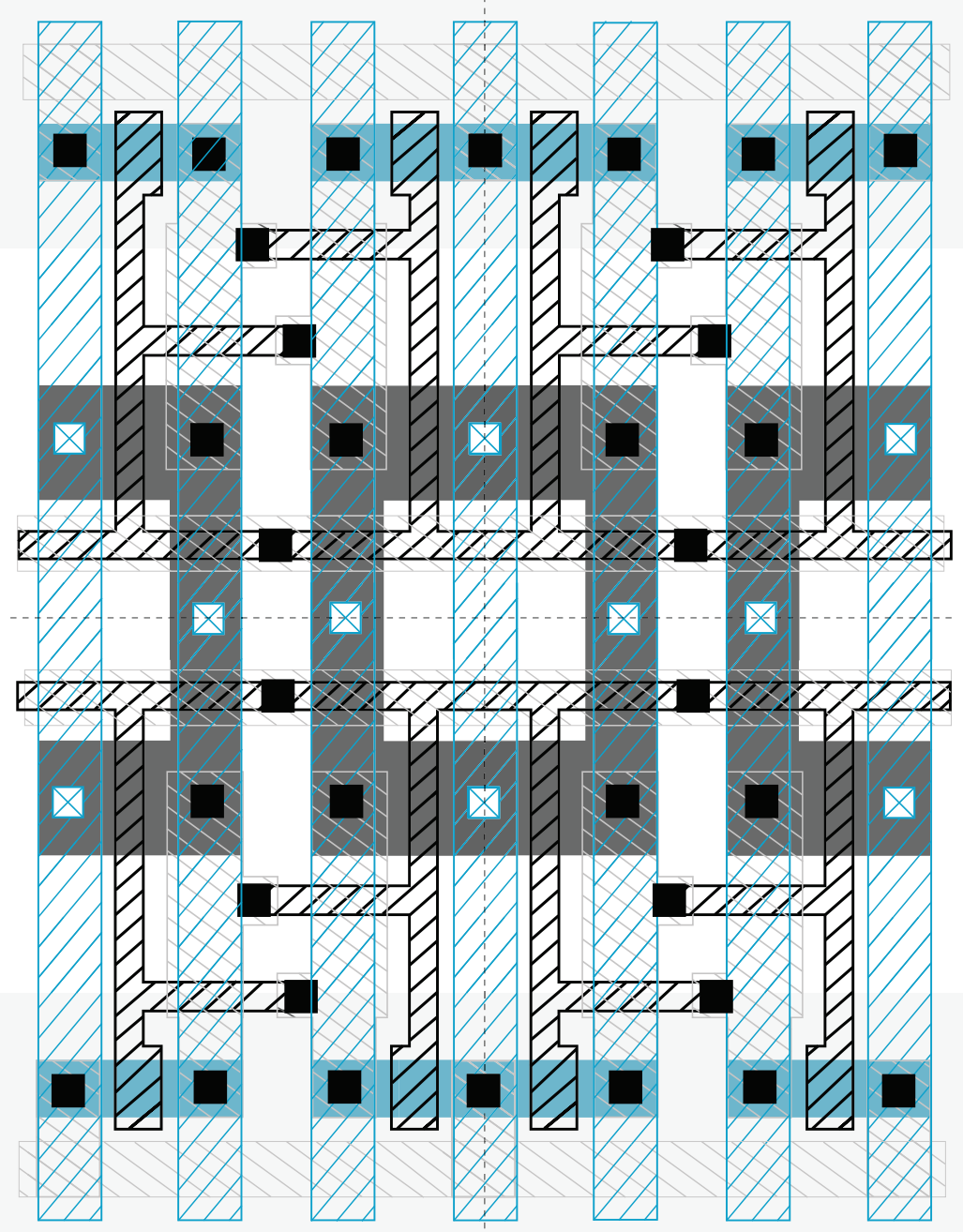

Figure 2.27: Arrayed historical layout of 6T SRAM cell [2].

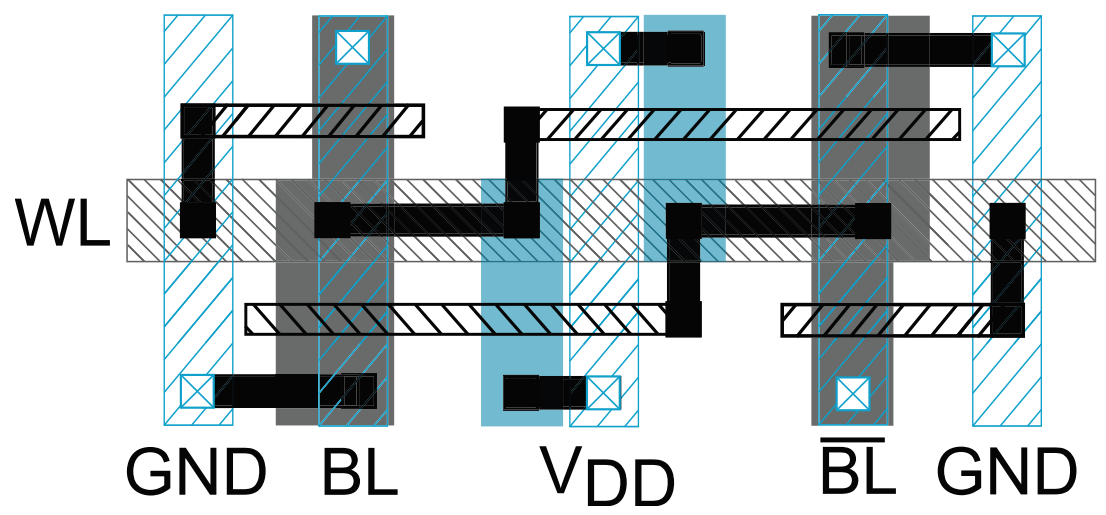

Figure 2.28: Lithographically friendly thin cell $6 \mathrm{~T}$ cell layout [2]. 


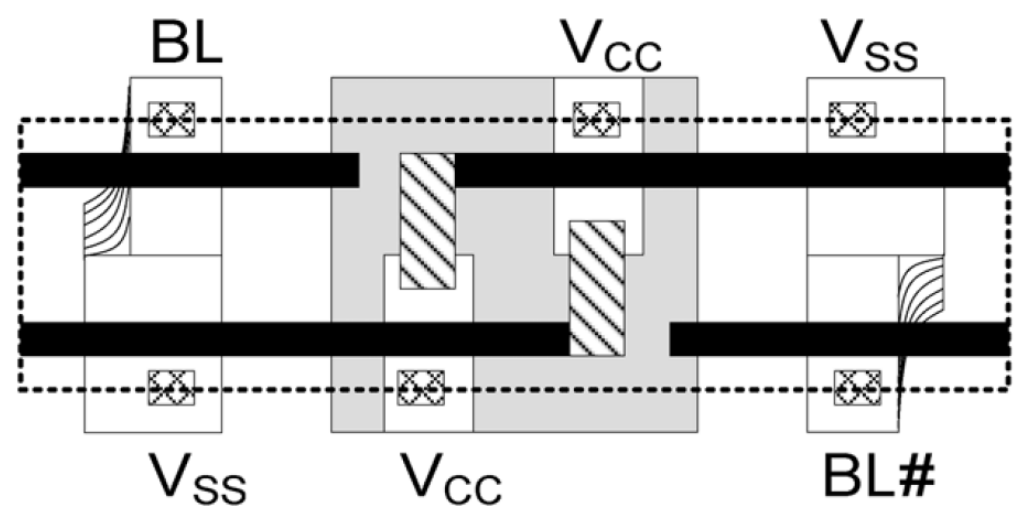

Figure 2.29: Thin cell $6 \mathrm{~T}$ cell layout showing rounding of the notch [9]. (C)2009 IEEE

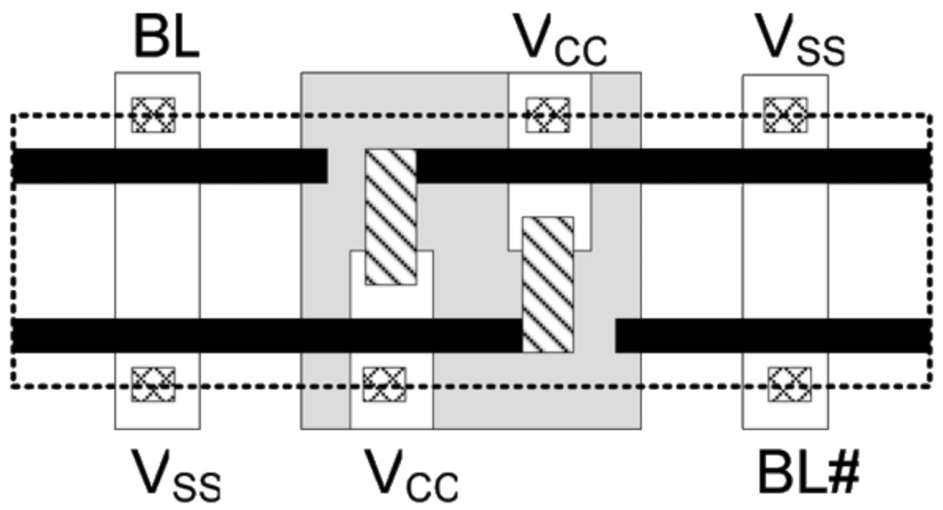

Figure 2.30: Diffusion-notch-free, or rectangular-diffusion, layout of 6T SRAM cell. Note how all transistors are sized equally [9]. (C)2009 IEEE 


\section{Chapter 3}

\section{Literature Review}

Many alternative architectures have been proposed to overcome the problems of the 6T SRAM cell at sub-threshold operation. Many studies have addressed the read stability issue by creating separate read and write paths, similar to the 8T SRAM cell. This allows for the optimization of each operation [6], [30], [31]. However, it practically always comes at the cost of additional transistors in the cell.

Other studies have focused on improving the traditional $8 \mathrm{~T}$ bit cell by utilizing the reverse short-channel effect [32], or by implementing additional circuitry [25] to further increase stability and allow lower voltage operation. Some have even focused on entirely different cell structures by using dynamic threshold voltage MOSFETs (DTMOS) [33], [34], or Schmidt triggers [35].

Many other papers focus on improving the write ability of the SRAM cell. Most of these investigations focus on making the storage cell weaker or access devices stronger. Reducing/floating the supply, and increasing/floating the ground voltage are common ways to make the cell weaker [25], [22], [36]. Breaking the feedback loop of the decoupled inverters has also been explored [37], [38]. However, both these methods affect the stability of half-selected cells. Making access devices stronger is a more promising approach. Upsizing access transistors ( [30], [39]), and boosting the word-line voltage ( [25] [12]) are good ways to achieve this, but the effect of upsizing transistors is lessened in sub-threshold operation, and boosting voltage requires additional circuitry.

Papers have also investigated ways to maintain the stability of half-selected cells. Some have prevented word-line sharing among cells in the same row by utilizing sub-rows [25]. Another research group has implemented a cross-point selection so there are no half-selected cells, but this requires two additional transistors for cell 
selection [13], [40]. One method designed to overcome this problem is a write-back operation in which the unaccessed cell's data is written back into it at the expense of speed and power [31], [41].

Extensive research has also been done to reduce bit-line leakage. Some studies have utilized replica bit-lines [32]. Others have added additional transistors to the bit cell [20], [31]. Many studies look to virtual grounds to reduce leakage [25], [22]. A few others use negative word-line [38] and bit-line [13] voltages.

The common theme among all these advancements is that they all increase the transistor count and/or power consumption. This results in a substantial increase in cost. However, there have been a small number of studies that attempt to reduce the total transistor count. In the following, some of the important previous work relevant to the topic of this thesis are presented.

\subsection{A Variation-Tolerant Sub-200 mV 6-T Sub- threshold SRAM}

A few designs have adapted the standard $6 \mathrm{~T}$ cell for use at sub-threshold voltages. One of the first of these designs was a single-ended $6 \mathrm{~T}$ bit-cell proposed in [10]. This design, presented in Figure 3.1, employs a full transmission gate in the bit-cell to drive the bit-line from rail to tail. Hence, the need for a sense amplifier is eliminatedreducing the chance of variability problems common in differential designs. Since the architecture is single-ended, noise is isolated to one bit-line, making it more robust to read upsets compared to differential designs. To overcome the reduced write margins associated with single-ended architectures, $V_{D D}$ and GND connected to the feedback inverter in the SRAM cell are drooped during a write. The combination of these design changes effectively decouples the read and write operations.

In order to overcome random dopant fluctuations causing $V_{t h}$ mismatch, the authors had to oversize the transistors. This increased the area to two times that of a standard $6 \mathrm{~T}$ cell. They did note however that device sizes could be reduced if a less stringent supply voltage floor is required.

It was determined by Monte Carlo simulations that at 16 bit-cells per bit-line the read current of a single bit-cell is always greater than the cumulative leakage current from the unaccessed cells. As seen in Figure 3.2, adjustable strength header 

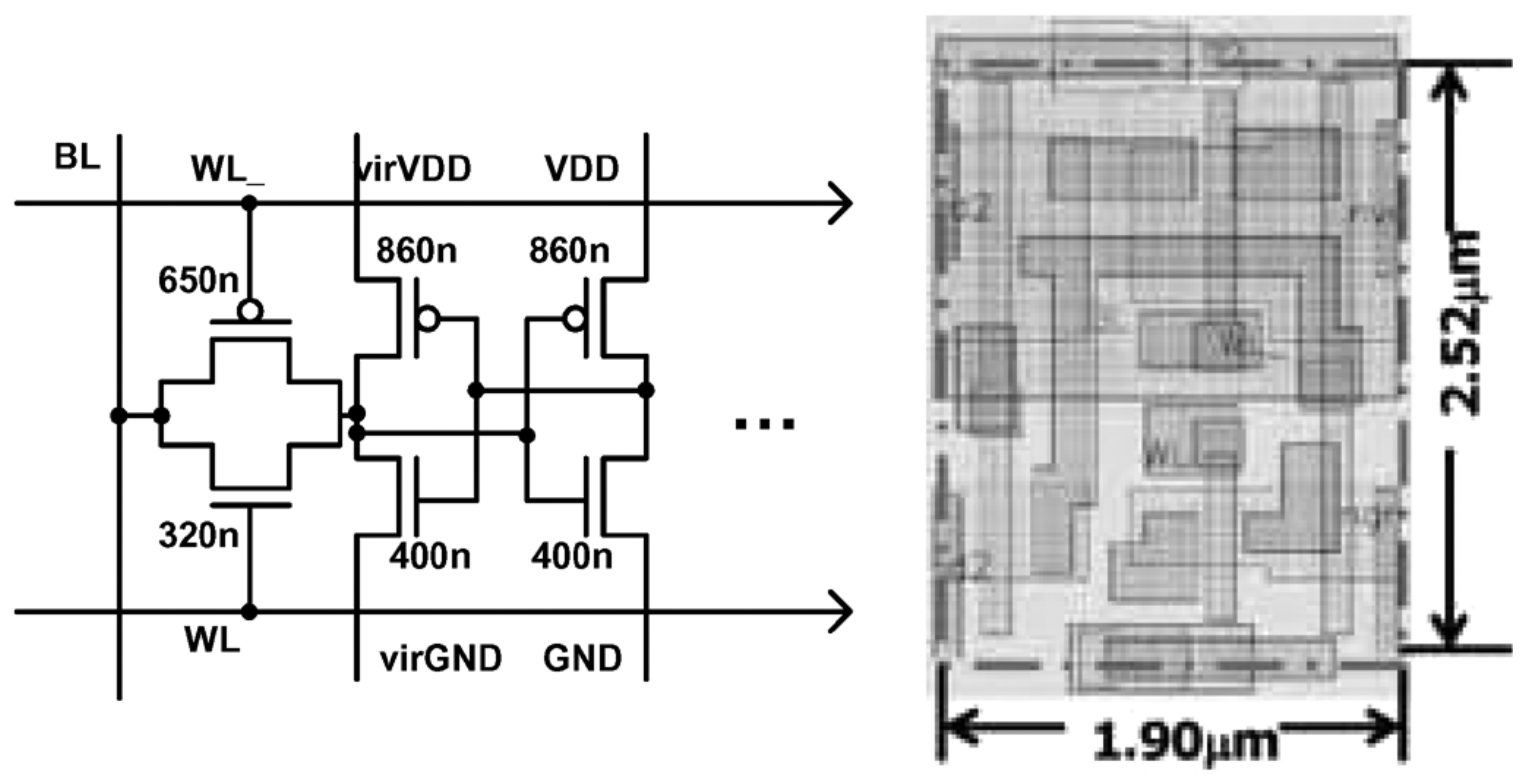

Figure 3.1: SRAM Cell design and area [10]. (C)2008 IEEE

and footer devices are used. When the wr_en signal is asserted, the strong PMOS transistor at the top and the strong NMOS transistor at the bottom are turned off. However, the weak headers and footers are still enabled, resulting in a voltage droop in VirVDD and VirGND that effectively disables the feedback inverter in the cell. This results in an increase in write SNM, but reduces the hold SNM of unaccessed cells that share the same VirVDD and VirGND. Rather than sharing the virtual GND and/or $V_{D D}$ with the entire row, which is typically done, the virtual GND and $V_{D D}$ are only shared by cells on the same local bit-line. While this increases the area, the hold SNM of unaccessed cells is only affected in cells on the same local bit-line rather than the entire row [10].

\subsection{Differential 6T Sub-threshold SRAM with Low Energy and Variability Resilient Local Assist Circuit}

The design in [10] was arguably improved upon in [11] by employing a differential bitline. Driving the bit-line from rail to rail was a promising approach to eliminate the read disturb issue, but required large transistors. They determined that a differential 


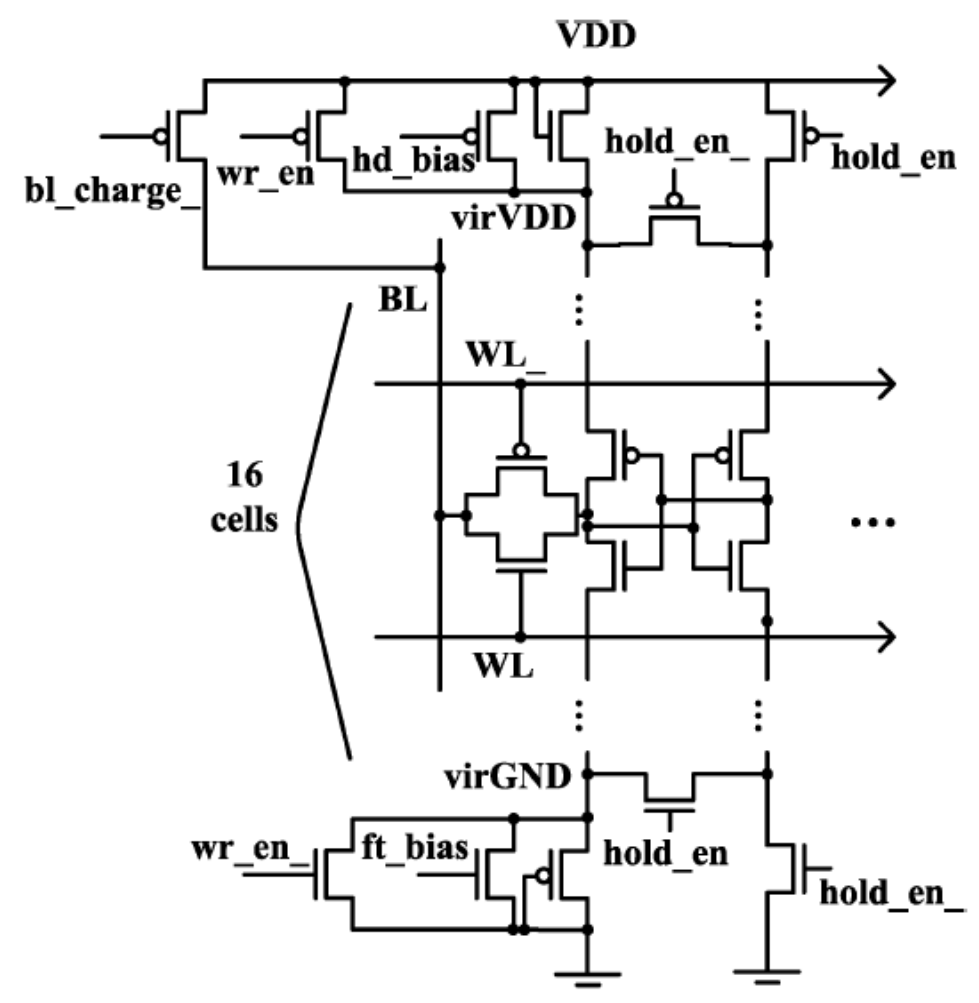

Figure 3.2: Cell array structure [10]. (C)2008 IEEE

bit-line would allow them to keep the transistor sizes of the bit-cells as small as possible.

Figure 3.3 shows the proposed SRAM architecture presented in [11]. Minimum sized high threshold voltage (HVT) transistors for each standard 6T bit-cell were utilized to reduce the leakage current. Each local bit-line is comprised of 16 bit-cells and a local sense amplifier. To reduce the bit-line energy consumption, a chargesharing based pre-charge scheme is used. When a cell in the column is not being accessed, the charges on bit-line (LBL) is shared with it's compliment (LBLB). This results in the bit-lines holding a charge somewhere between $V_{D D}$ and GND. This also has the added advantage of reducing the read disturb voltage, and, thus, improving the read stability.

Typically, a differential bit-line cannot guarantee functionality at ultra-low voltages due to process variations [42]. To combat process variations, a transmission gate is used instead of the typical pass transistor to link between the local bit-line and 


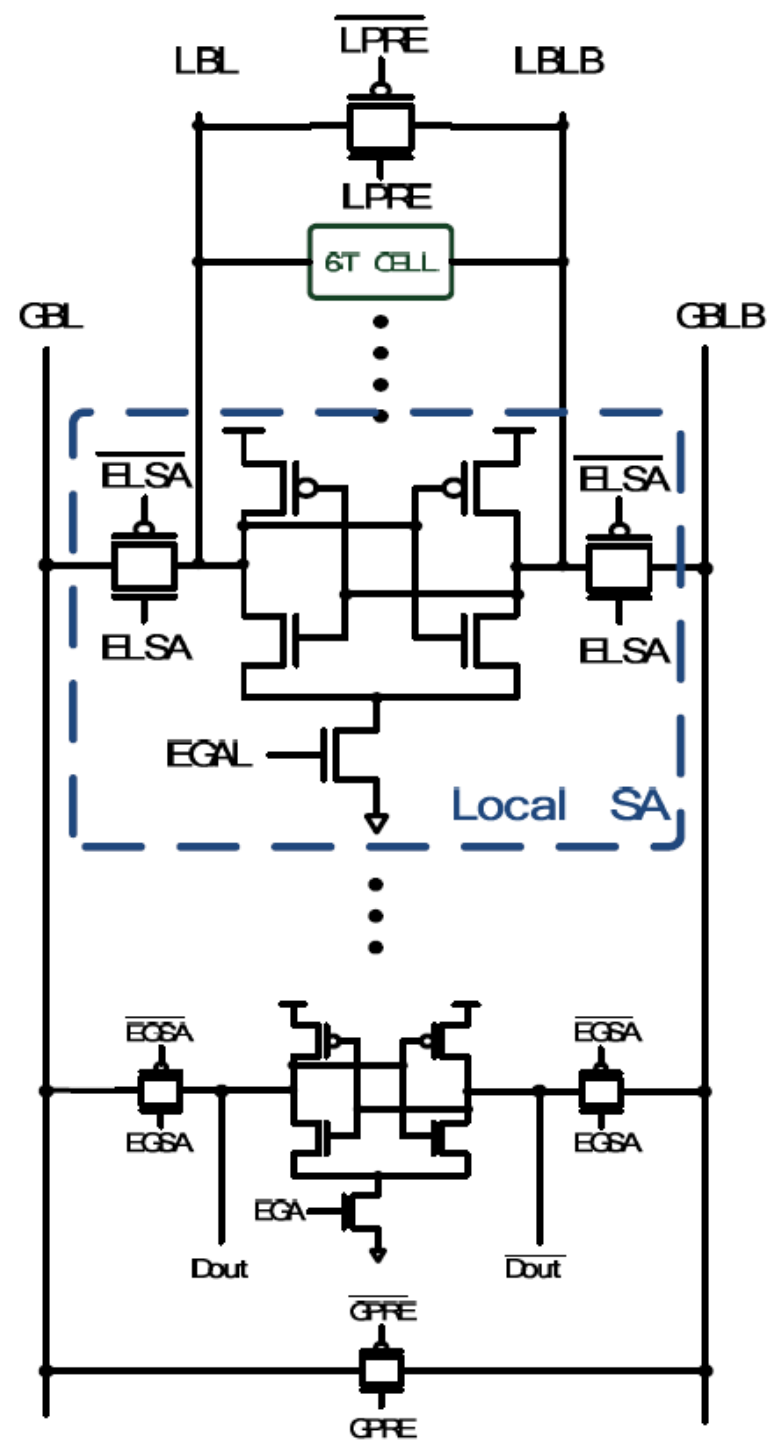

Figure 3.3: Chiou et al.'s proposed SRAM architecture [11]. (C2013 IEEE

global bit-line. The extra driving current suppresses the intra-die variations. However, this comes at the cost of additional area. With a sense amplifier required for every 16 bit-cells, the required area is vastly increased compared to a standard $6 \mathrm{~T}$ SRAM architecture. 


\subsection{Average-8T Differential-Sensing Subthreshold SRAM With Bit Interleaving and 1k Bits Per Bitline}

While the previous two studies succeeded in adapting the 6T SRAM cell for subthreshold operation, they dramatically increased area consumption. The design proposed in [12] presents a sub-threshold differential SRAM architecture with a robust read scheme and minimum area. The SRAM architecture is illustrated in Figure 3.4.

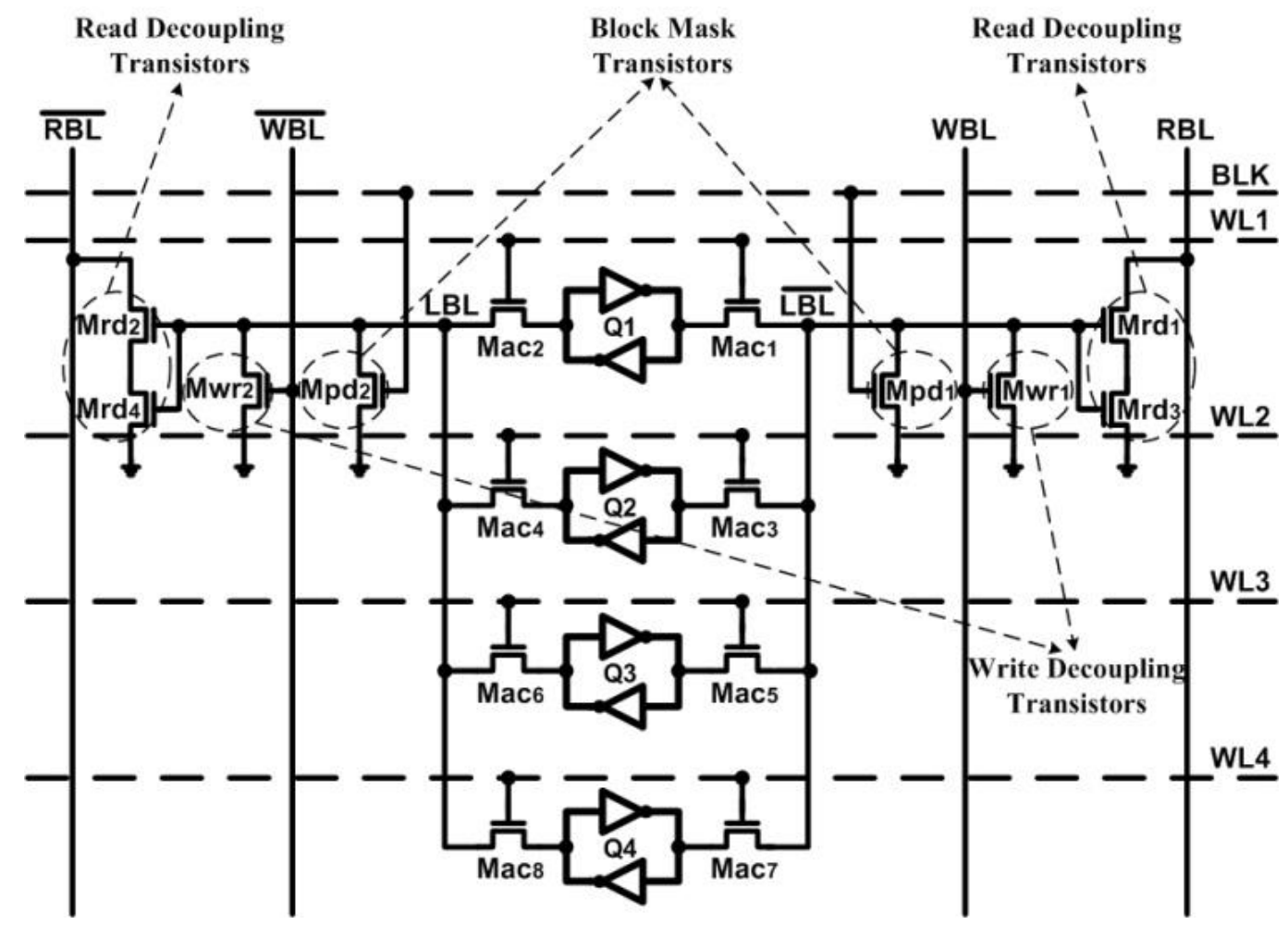

Figure 3.4: Khayatzadeh and Lian's proposed SRAM cell [12]. (C2013 IEEE

The design proposes having cell blocks that vary in transistor count depending on how many bits are stored in each block (up to 16 bits). The fewer the number of bits, the more tolerable it is of lower voltages. This varies the average transistor count from 14 ( 1 bit per block) to 6.5 (16 bits per block). The study focuses on the average 8 transistor case since that is the closest to the conventional $8 \mathrm{~T}$ architecture.

As shown in Figure 3.4 that the design utilizes the standard 6T architecture with localized bit-lines. These local bit-lines are decoupled from the global read and write 
bit-lines by $M r d_{1-4}$ and $M w r_{1-2}$ respectively. In the idle/unselected state (wordlines are disabled for the block), the local bit-lines are left floating. This may turn on $\mathrm{Mrd}_{1-4}$ and possibly disturb the read operation of another block. To resolve this issue, block mask transistors $\left(M p d_{1-2}\right)$ are utilized to keep the local bit-lines low and minimize leakage. Figure 3.5 illustrates this unselected/idle state.

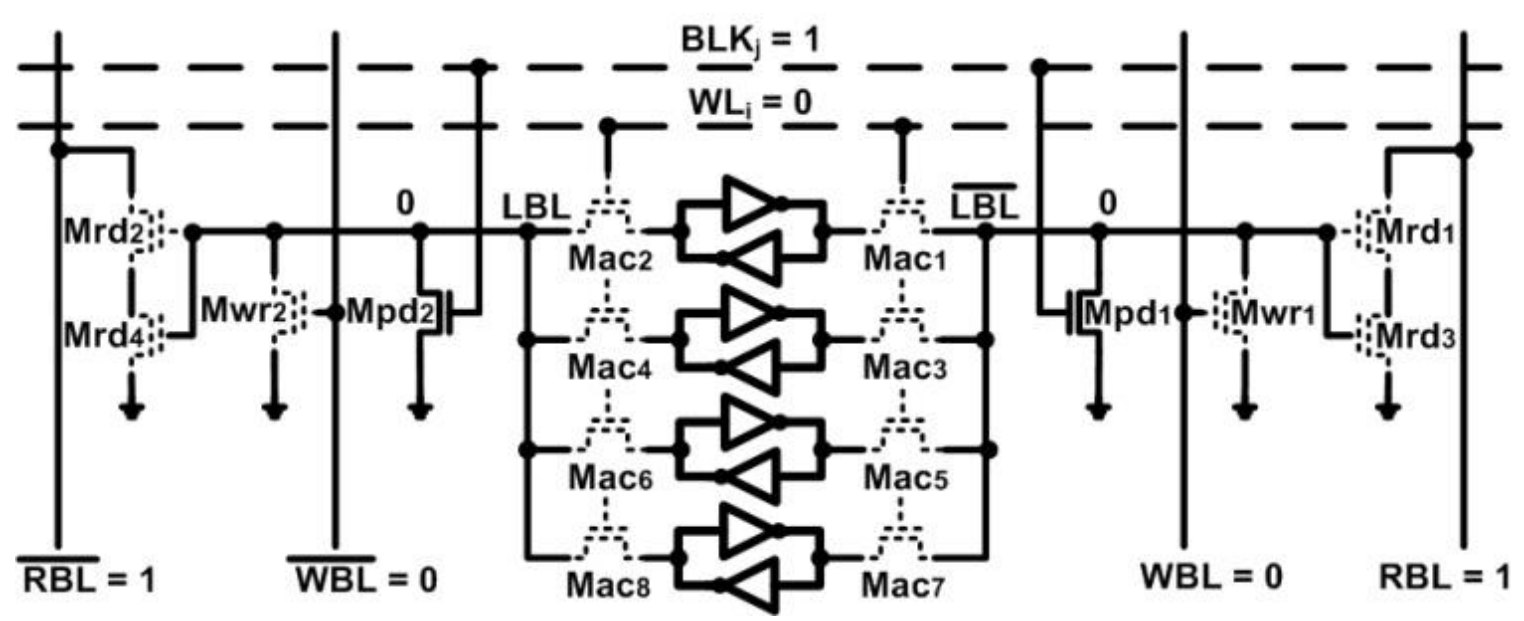

Figure 3.5: Khayatzadeh and Lian's proposed SRAM cell in the hold state [12]. (C) 2013 IEEE

When in the idle/standby state, leakage and power can be further reduced by turning off the block mask transistors. This results in the local bit-lines being left floating. Additionally, the global read bit-lines are set to a high impedance mode by disabling the pull-up networks for the global read bit-lines.

While this design is similar to the standard 6T SRAM design with hierarchical bit-lines, it separates itself by having data-independent leakage, separate differential read and write bit-lines, and decoupled write operation. Data-independent leakage is a result of the block mask transistors keeping the local bit-lines low when unselected. This reduces the leakage during reads. Leakage is further reduced by the stacked read decoupling transistors.

While the storage cell is a standard 6T SRAM cell, the read operation is slightly different, as illustrated in Figure 3.6. Similar to the standard case, the read operation is done by raising the word-line connected to the access transistors of the desired cell. However, since the local bit-lines are not pre-charged high like in the standard case, 


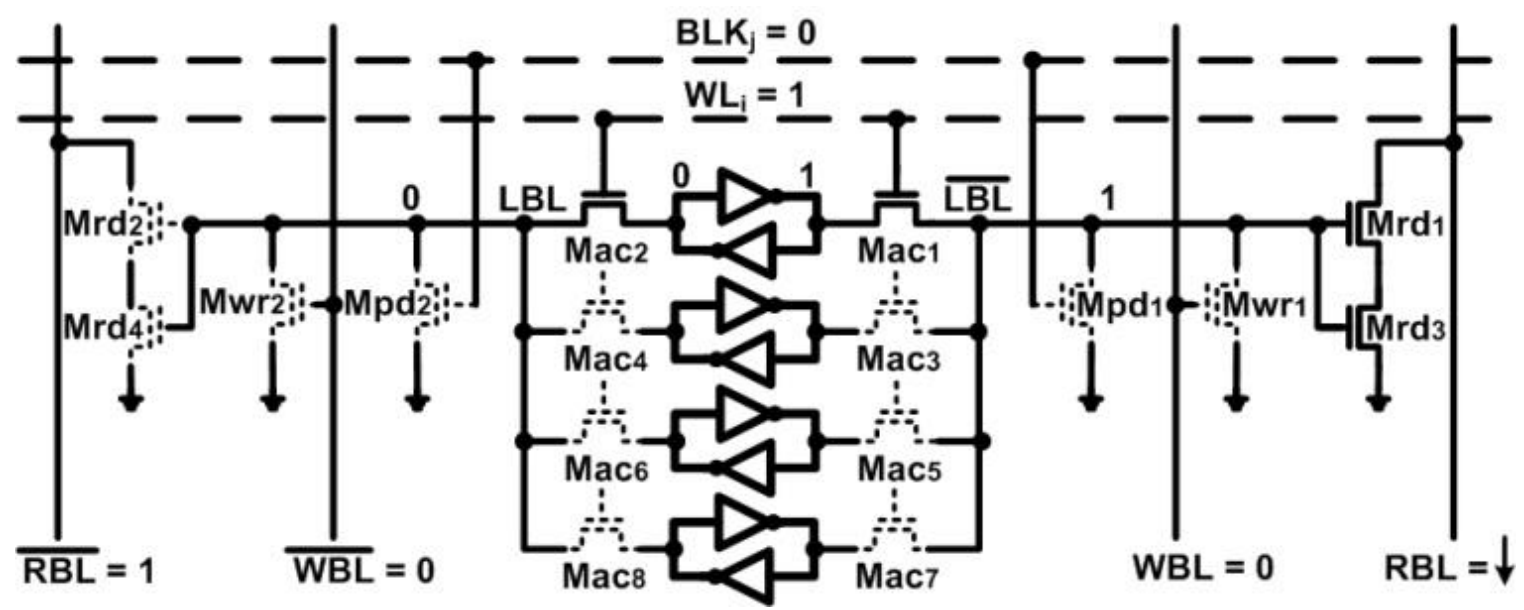

Figure 3.6: Khayatzadeh and Lian's proposed SRAM cell in the read operation [12]. (C) 2013 IEEE

the stored data sets the values of the local bit-lines. The high local bit-line enables the read decoupling transistors, discharging one of the pre-charged high global read bit-lines. It's important to note that the value of the high local bit-line will not fully be $V_{D D}$ since the access transistors are NMOS transistors. This can be mitigated by boosting the word-line voltage during reads, but at the cost of a more complex power supply and increased area consumption.

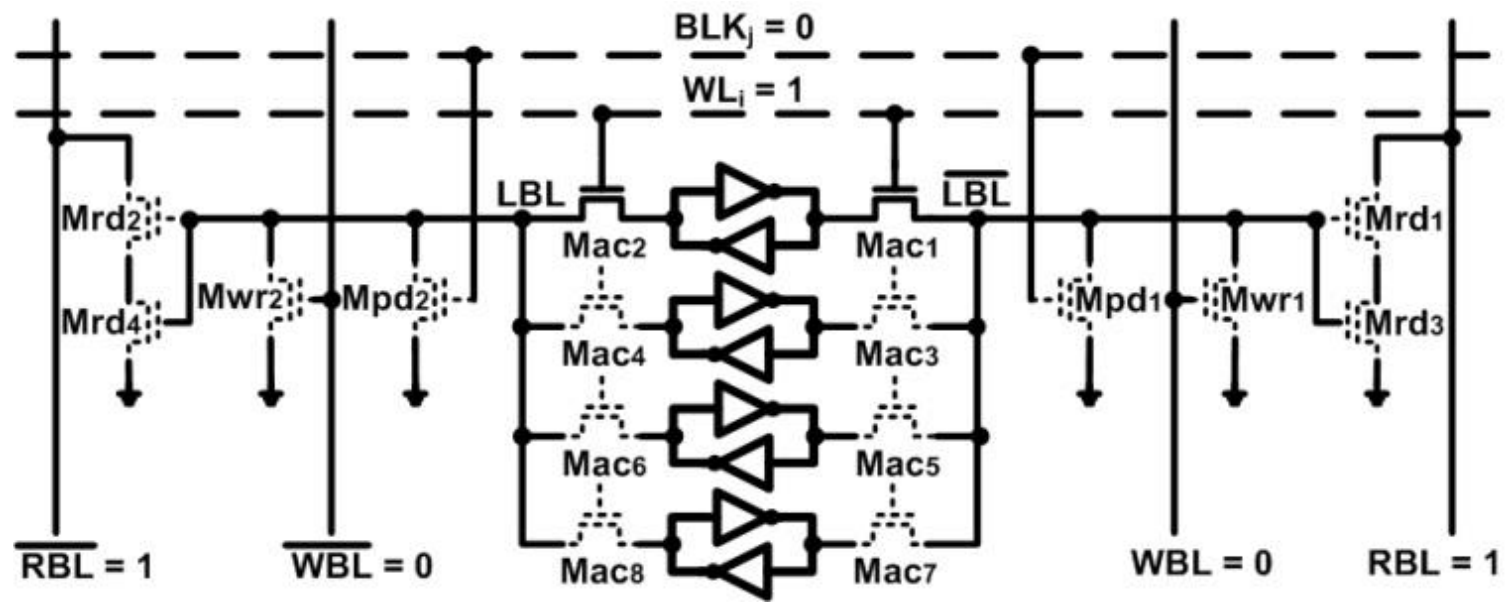

Figure 3.7: Khayatzadeh and Lian's proposed SRAM cell when half-selected [12]. (C) 2013 IEEE 
Figure 3.7 depicts the half-selected cells in the same row (access transistors are on). These half-selected cells are relatively stable since all pull-down transistors are off, and the local bit-line is very short. Thus, the disturbance caused by the predischarged local bit-line capacitance is relatively small.

The downside of this relatively complex read scheme is the that the timings of the BLK and word-line signals must be carefully designed to not overlap. If the access transistors are enabled before the block mask transistors are turned off, it is possible for the data in the storage cell to be overwritten.

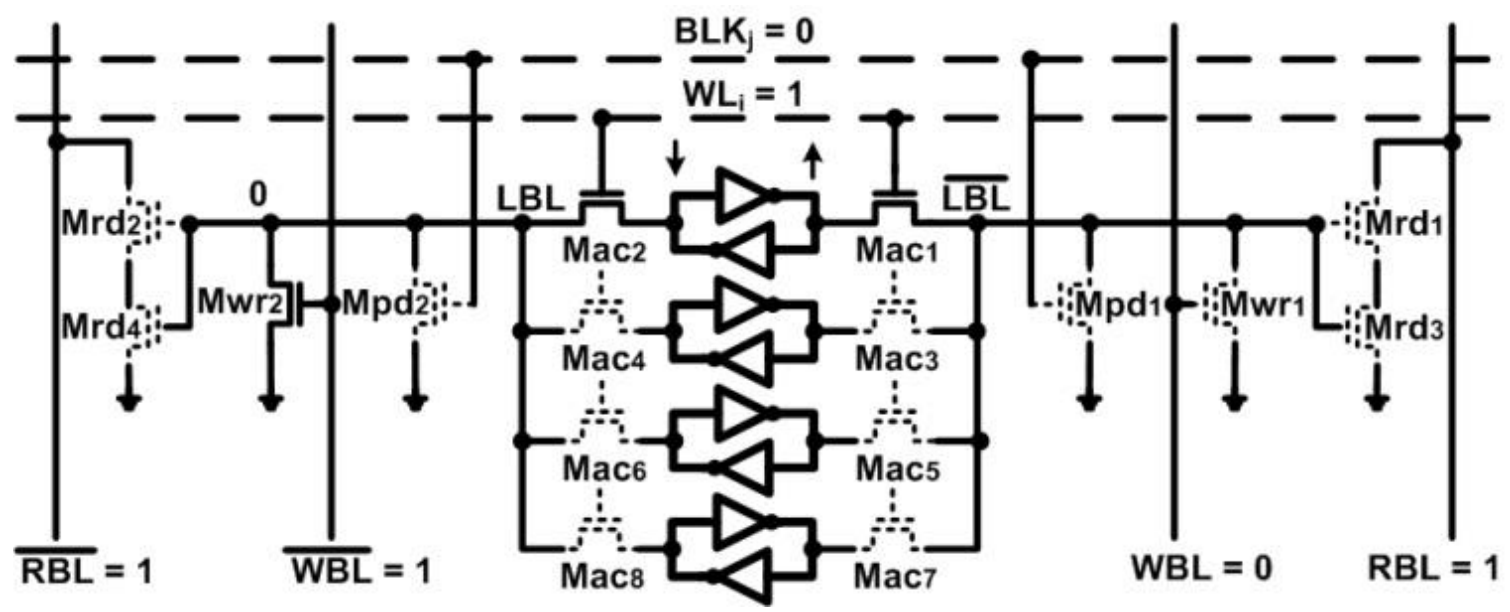

Figure 3.8: Khayatzadeh and Lian's proposed SRAM cell in the write operation [12]. (C) 2013 IEEE

The write operation is shown in Figure 3.8. The basic mechanism of the write operation is the same as in the standard 6T SRAM case. The word-line for the selected row is raised high, and, depending on the data to be written, one of the local bit-lines is discharged. However, since the write operation is single ended, the WSNM suffers compared to the standard $6 \mathrm{~T}$ case. The half-selected case is the same as in the read operation; there is relatively little disturbance from the local bit-line capacitance. 


\subsection{A Single-Ended Disturb-Free 9T Subthreshold SRAM With Cross-Point Data-Aware Write Word-Line Structure, Negative Bit-Line, and Adaptive Read Operation Timing Tracing}

A very interesting SRAM architecture is proposed in [13], as depicted in Figure 3.9. Rather than decreasing area, they aimed to improve read stability with a read buffer and eliminate half-select disturb during, writes while utilizing a single bit-line to improve density and reduce BL power dissipation. Previous studies (e.g. [43], [28], [44], [40], [8]) have shown that write half-select disturb can be eliminated by utilizing a cross-point write structure. In a cross point structure, both row and column based word-lines are used in conjunction to select a cell. The word-line (WL) and virtual VSS (VVSS) are row-based, and the write word-lines (WWLA and WWLB) and bitline (BL) are column-based. The timing diagram for each operation can be seen in Figure 3.10.

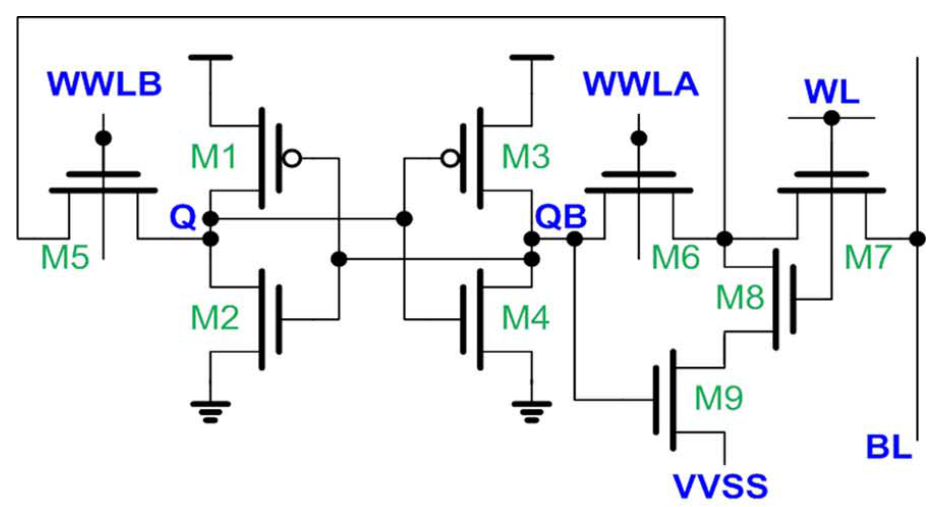

Figure 3.9: SRAM cell proposed in [13]. (C)2012 IEEE

Reading is performed somewhat similar to the standard 8T SRAM cell case. The read word-line (WL) is set high and the write word-lines remain disabled, in addition to virtual VSS (VVSS) being forced to ground. Transistors M7-M9 buffer the data during reads to conditionally discharge the BL. Since Q and QB are isolated from the bit-line during reads, the read SNM is very close to the hold SNM. Additionally, unselected cells sharing the same bit-line have greatly reduced leakage. This is due 


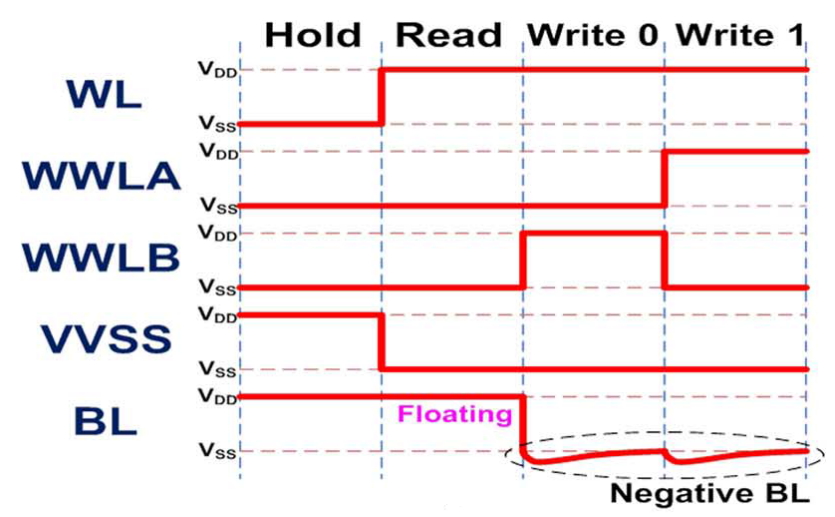

Figure 3.10: Timing diagram of the 9T SRAM cell designed in [13]. (C)2012 IEEE

to the unselected cells in the same column having their VVSS held at $V_{D D}$ and the stacking of the transistors in the read buffer.

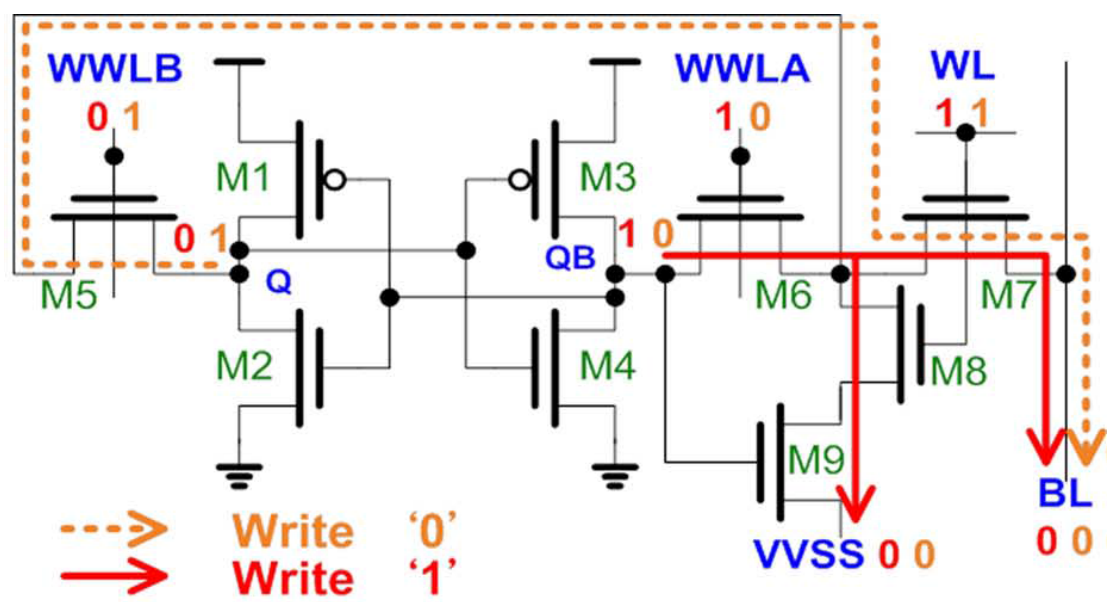

Figure 3.11: Write operation of the 9T SRAM cell presented in [13]. (C)2012 IEEE

One of the most innovative features of this design is its write operation. Figure 3.11 illustrates how the write operation works. When writing a ' 1 ' into the cell, WL and WWLA are brought high while VVSS and BL are forced to ground, and WWLB remains low. This causes QB to discharge by the bit-line (through M6 and M7) and by VVSS (through M8 and M9), setting QB to ' 0 ' and Q to ' 1 '. When writing a '0', WL and WWLB are set high while VVSS and BL are forced to ground, and WWLA remains low. This causes $\mathrm{Q}$ to discharge by the bit-line (through M5 and M7).

It is important to note that the write ability is degraded by the series-connected 
NMOS transistors (M5/M6 and M7). The authors alleviated this by utilizing a negative bit-line during writes. However, this requires additional area and a more complex power supply.

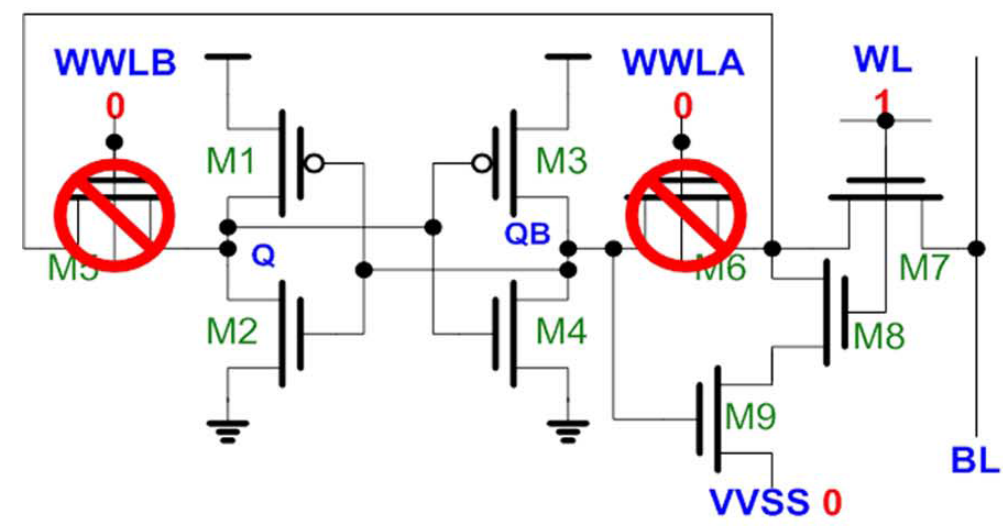

Write Half-selected cells in the active row

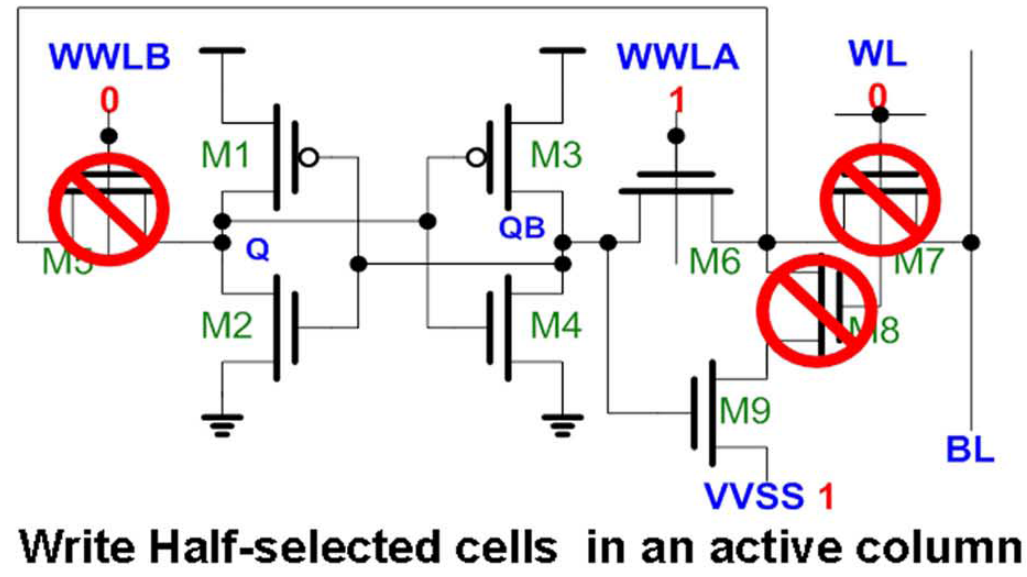

Figure 3.12: Half-selected rows during the write operation of the 9T SRAM cell presented in [13]. (C)2012 IEEE

For a write to occur, WL and one of the write word-lines must be enabled to change the data in the cell. Since WL and VVSS are row-based and the write wordlines (WWLA and WWLB) are column-based, it is not possible change the value of a half-selected cell, as depicted in Figure 3.12. In half-selected cells within the active row, the access transistors are disabled. In half-selected cells within the active column, WL is disabled and VVSS is high (effectively eliminating the leakage current through M9 and M8). As a result, nodes Q and QB in half-selected cells in both the active row and column are isolated. Having half-select disturb-free writes allows for 
a bit-interleaving architecture.

Most architectures that utilize read buffers require separate bit-lines for the read and write operations. However, this architecture is able to utilize the same bit-line for reads and writes. This allows for power savings as the number of bit-line charges and discharges are reduced.

Perhaps the most interesting aspect of this design is the vertical word-lines (WWLA and WWLB) connected to the gates of the access transistors. These vertical word-lines allow each side of the back-to-back inverters ( $Q$ and QB) to be pulled down to 0 using the same bit-line. In the typical case of horizontal word-lines, this is not possible since the bit-lines set the values of the cells and/or both access transistors would be enabled at the same time.

A big downside to this 9T SRAM design is the large area. There is a $97 \%$ area overhead over the 6T SRAM cell.

\subsection{Analysis of Past Research}

Previous studies have shown that a hierarchical bit-line approach utilizing the standard 6T SRAM cell is perhaps the ideal way to minimize area. However, the standard 6T SRAM requires differential bit-lines, which is arguably not ideal for subthreshold SRAM. A single ended approach while keeping the cell transistor count to $6 \mathrm{~T}$ or lower, requires the ability to strongly write a ' 1 ' by overcoming the stronger NMOS pull-down (or load) transistors (such as through the use of large transmission gates in [10]), or a scheme that allows the writing of a ' 0 ' to each side of the storage cell.

The SRAM architecture presented in [13] provides a method to do the latter. By utilizing vertical word-lines and connecting the access transistors to the same bit-line, it is possible to discharge the value stored on each side of the storage cell-writing a ' 1 '. However, their designed scheme requires a separate read path utilizing more transistors. The Designs in [10], [11], and [12] all feature hierarchical bit-lines with the same read and write paths, saving on area. Maybe combining the two schemes would present an ideal SRAM architecture.

However, utilizing vertical word-lines along with traditional vertical bit-lines presents a problem when selecting cells. Enabling a vertical word-line will select every cell in that column. During a write, the stored value in every selected cell is affected. During a read, the stored value in each selected cell affects the read value. 
The design in [13] overcame this problem by having an additional horizontal wordline, so that a cell can only be selected if the horizontal and vertical word-lines are enabled. This requires an additional transistor for each cell, and does not allow for good readability; during the read operation, the data has to pass through two NMOS transistors. Perhaps overcoming this issue may conceivably present a SRAM design that has high integration density. 


\section{Chapter 4}

\section{Proposed SRAM Architecture}

The chapter presents a novel SRAM architecture. Figure 4.1 shows the basic proposed SRAM in a 2-cell configuration. The SRAM cell is similar to the standard 6T SRAM cell, i.e. cross-coupled inverters are utilized for the storage cell along with 2 access transistors to retrieve or alter the data. However, a distinct difference from the standard $6 \mathrm{~T}$ cell is that the gates of the access transistors are not connected together, and the select word-lines are vertical rather than the traditional horizontal. This completely changes the read and write operations.

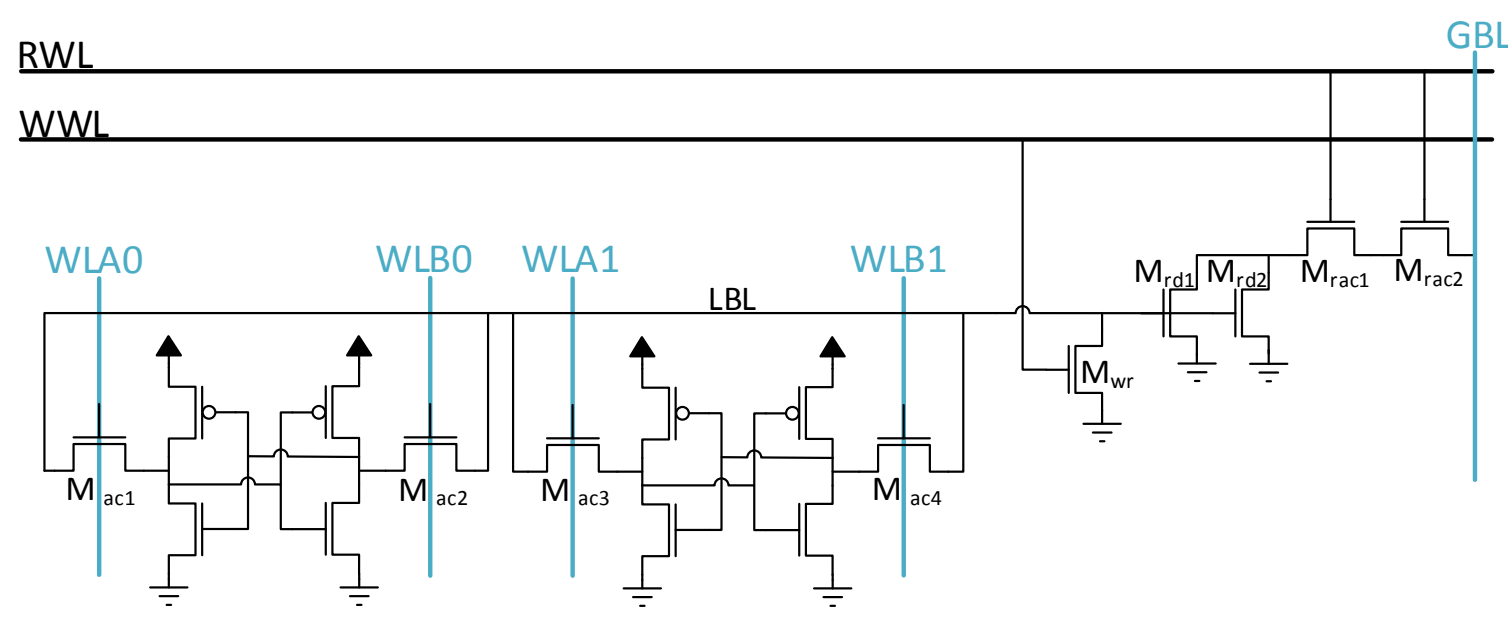

Figure 4.1: Basic architecture of the proposed cell block in a 2-cell configuration.

Both of the access transistors are connected to the same horizontal local bit-line (LBL). The local bit-line is decoupled from the vertical global bit-line by the readdecoupling transistors, $M_{r d 1}$ and $M_{r d 2}$, and the read access transistors, $M_{r a c 1}$ and 
$M_{r a c 2}$. The write operation does not utilize the global bit-line. Instead, the write operation is decoupled by $M_{w r}$. The word-lines for the the read and write operations are horizontal.

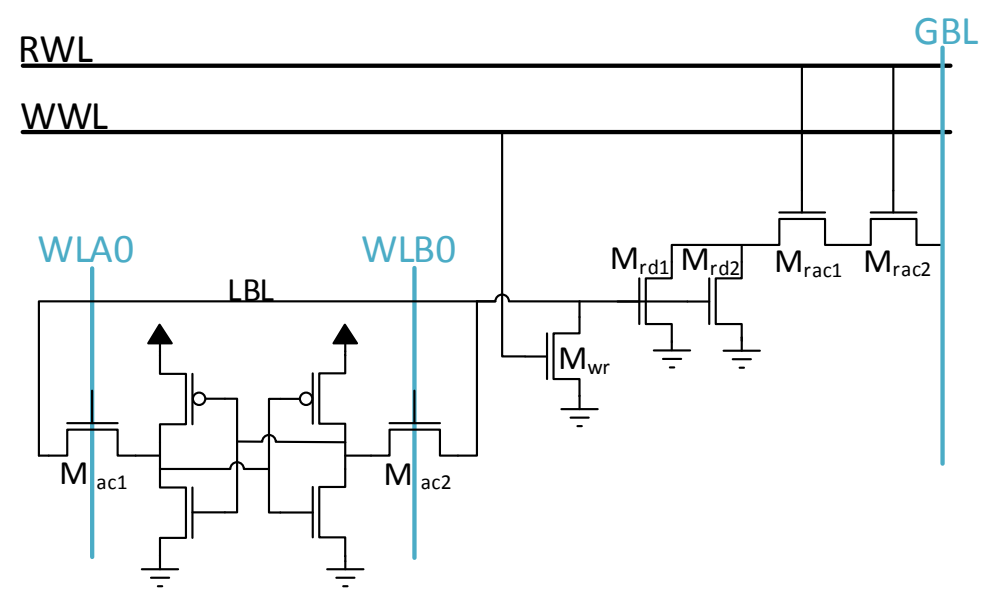

Figure 4.2: Schematic of the proposed cell block in a 1-cell configuration.

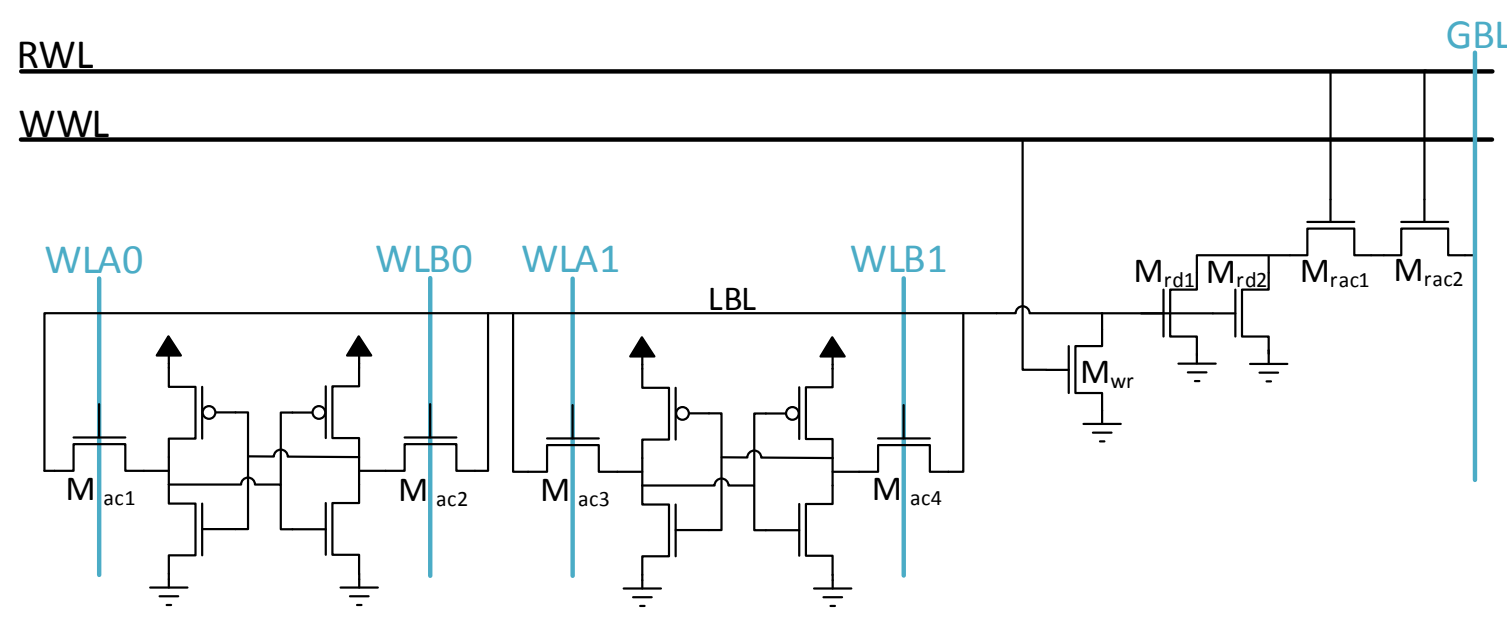

Figure 4.3: Schematic of the proposed cell block in a 2-cell configuration.

The number of transistors in each cell block varies depending on how many cells are in each block. Since the goal is to minimize area, it is desirable to maximize the number of bits in each the block. However, the minimum operational voltage 


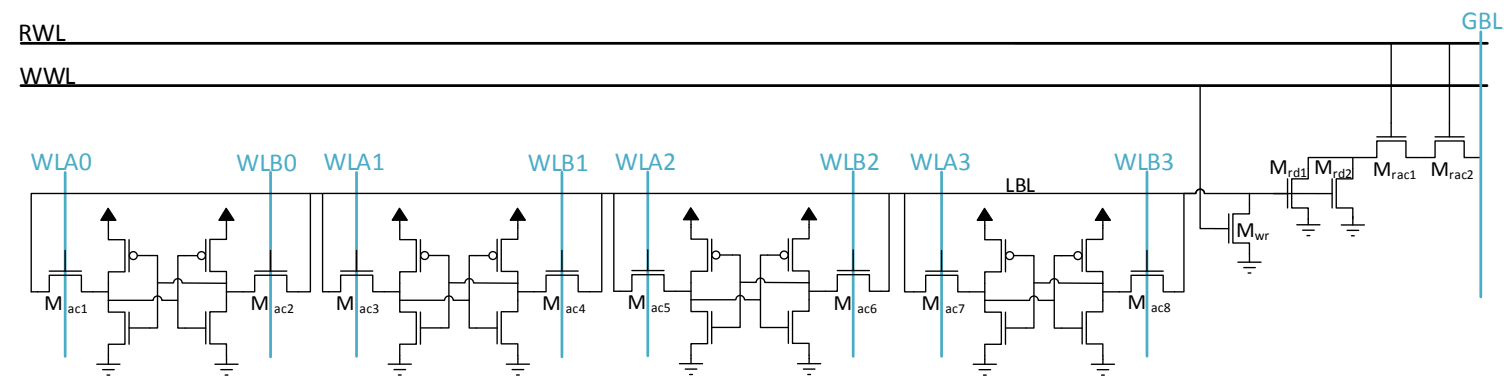

Figure 4.4: Schematic of the proposed cell block in a 4-cell configuration.

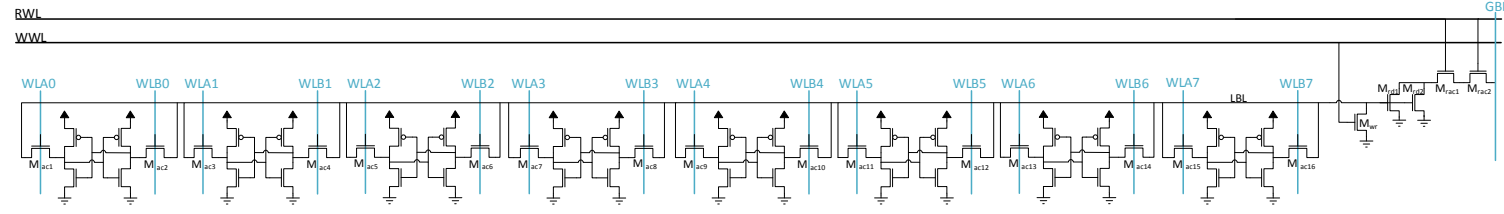

Figure 4.5: Schematic of the proposed cell block in a 8-cell configuration.

limits the number of bits that can be on the same bit-line due to bit-line leakage and relatively poor $I_{O N}$ to $I_{O F F}$ ratio. For clarity, this explanation will focus on a configuration of 2-cells in each cell block. The 1-cell configuration can be seen in Figure 4.2, 2-cell in Figure 4.3, 4-cell in Figure 4.4, and 8-cell in Figure 4.5.

\subsection{Write Operation}

The main rational for employing a horizontal local bit-line is for the write operation. As discussed in Section 2.1.2, data is written into standard SRAM cells by writing a ' 0 ' to a node in the storage cell. In traditional architectures, this means separate access transistors for each node connected to different bit-lines. When used in combination with vertical word-lines, horizontal bit-lines allow both access transistors to utilize the same bit-line in its write path. The allows for a decoupled write operation while keeping the transistor count to a minimum. A decoupled write operation isolates the cell node from the long write bit-lines, decreasing the vulnerability of noise.

Due to the vertical bit-lines, the traditional way of selecting a cell cannot be used. The access transistors of the standard $6 \mathrm{~T}$ cell is still used as a selection switch, but both access transistors cannot be enabled at the same time without contention. The 


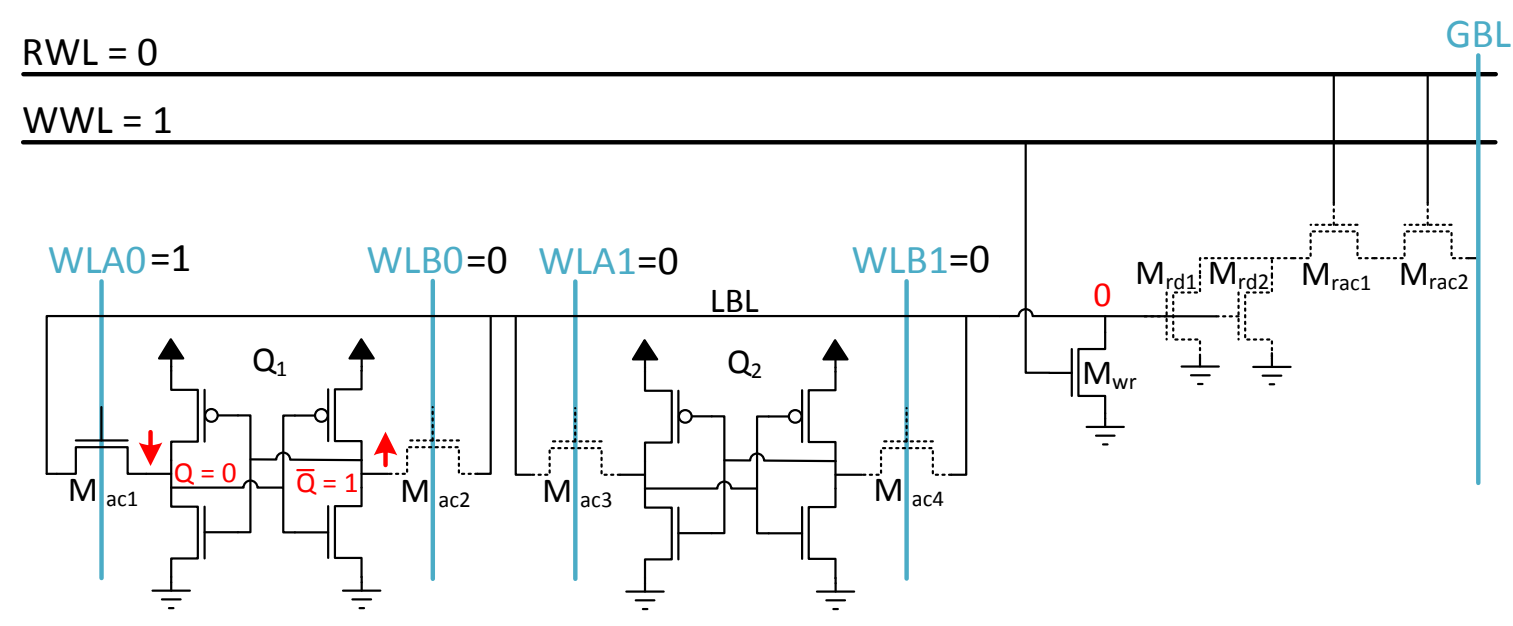

Figure 4.6: Write operation of the proposed SRAM design.

select word-lines connected to the access transistors (WLA and WLB) can analogously be compared to the write bit-lines, and the read and write word-lines (WWL and RWL) are basically identical to the read and write word-lines in the common $8 \mathrm{~T}$ SRAM architecture. Since the write operation is completely decoupled, the only bit-line used in the write operation is the local bit-line (LBL).

Cell selection in the write operation is done by enabling the write word-line (WWL) for the row containing the cell, and the respective select word-line, WLA (word-line A) or WLB (word-line B), is enabled for the cell's column, depending on the value being written. Enabling these word lines turns on $M_{w r}$, and $M_{a c 1}$ or $M_{a c 2}$. The selected node is then discharged through the enabled access transistor and $M_{w r}$.

Determining which select word-line is selected to write a ' 1 ' depends on which select word-line is used for reads. When using WLB for reads, selecting WLA during writes will write a ' 0 ' and WLB will write a ' 1 '. When using WLA for reads, selecting WLA during writes will write a ' 1 ' and WLB will write a ' 0 '. In the read configuration discussed in Section 4.2, WLB is used for reads. Thus, write operation discussed writes a ' 0 ' when WLA is selected, and a ' 1 ' when WLB is selected.

Figure 4.6 depicts writing a ' 0 ' into $Q_{1}$. The access transistor, $M_{a c 1}$, for $Q_{1}$ is enabled by WLA0, and the write decoupling transistor, $M_{w r}$, is enabled by WWL. Node $\mathrm{Q}$ is then discharged through $M_{w r}$. This results in node $\bar{Q}$ being forced to ' 1 ' through the feedback process by the cross-coupled inverters. The timing diagram for the operation of writing a ' 0 ' is illustrated in Figure 4.7. If writing a ' 1 ' is desired, 

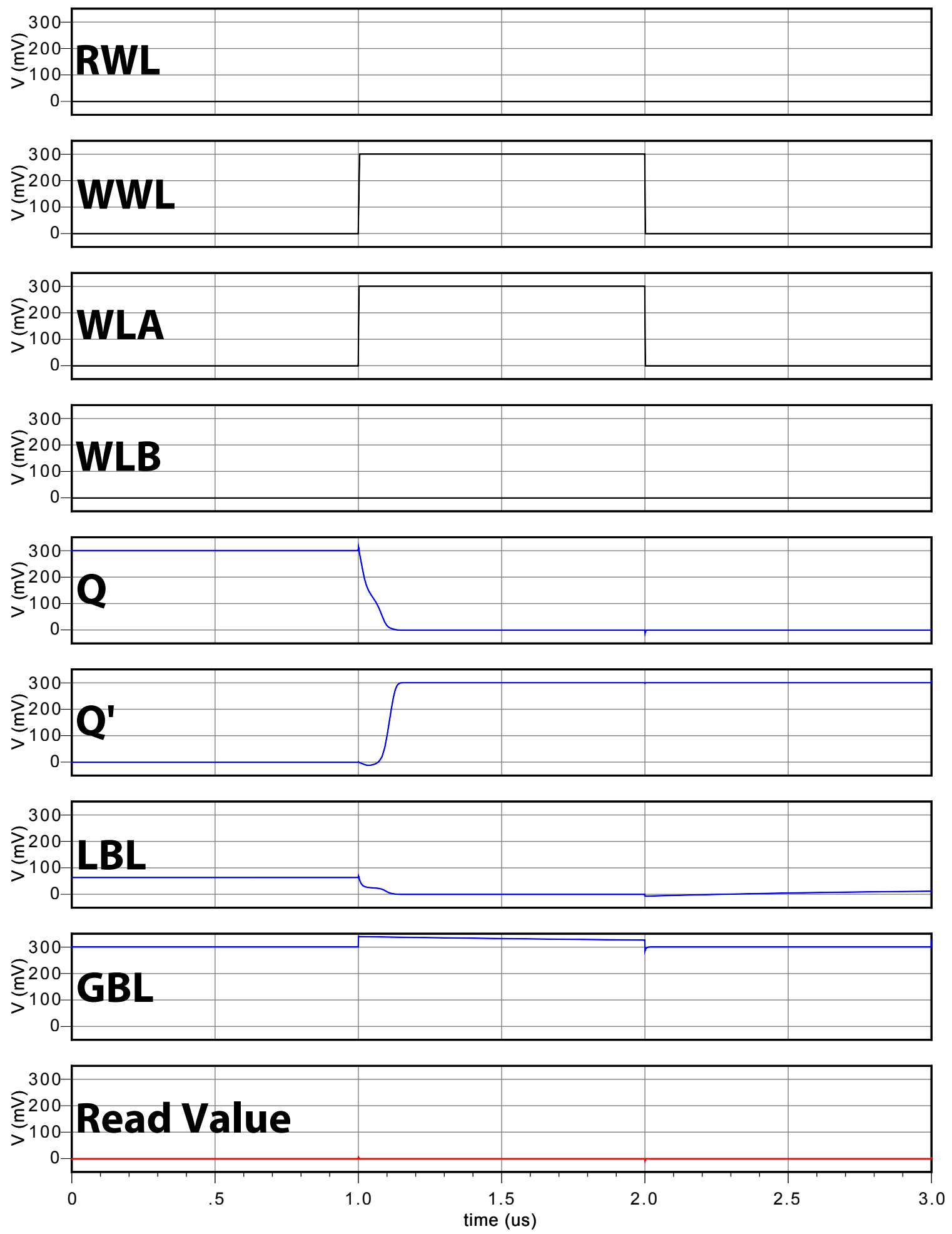

Figure 4.7: Timing diagram of the write operation when writing a ' 0 '. 

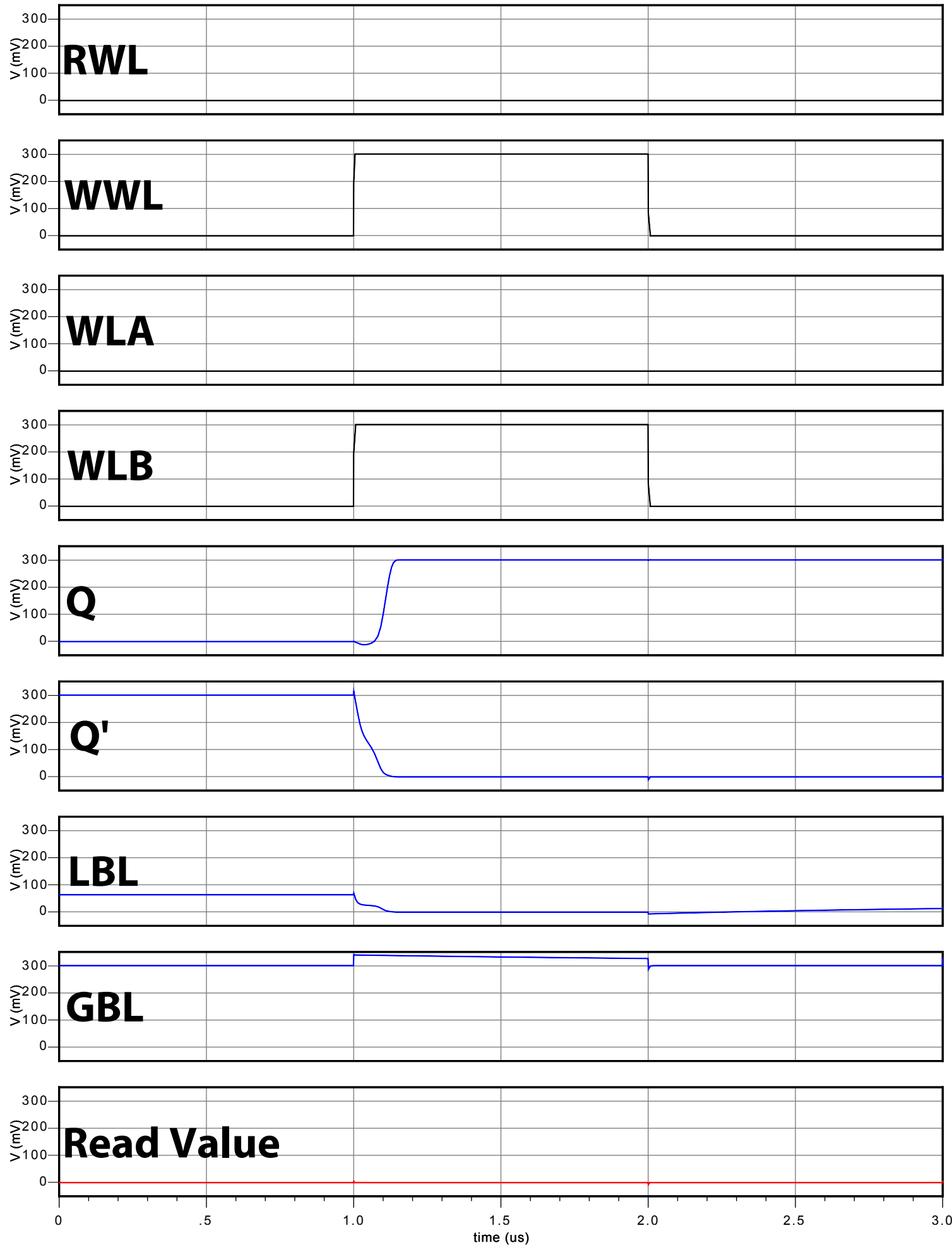

Figure 4.8: Timing diagram of the write operation when writing a ' 1 '. 
WLB0 for the cell should be enabled instead of WLA0. Otherwise, the write process is the exact same with Node $\bar{Q}$ being discharged through $M_{w r}$, and node Q being forced to ' 1 '. The timing diagram for the operation of writing a ' 1 ' is shown in Figure 4.8 .

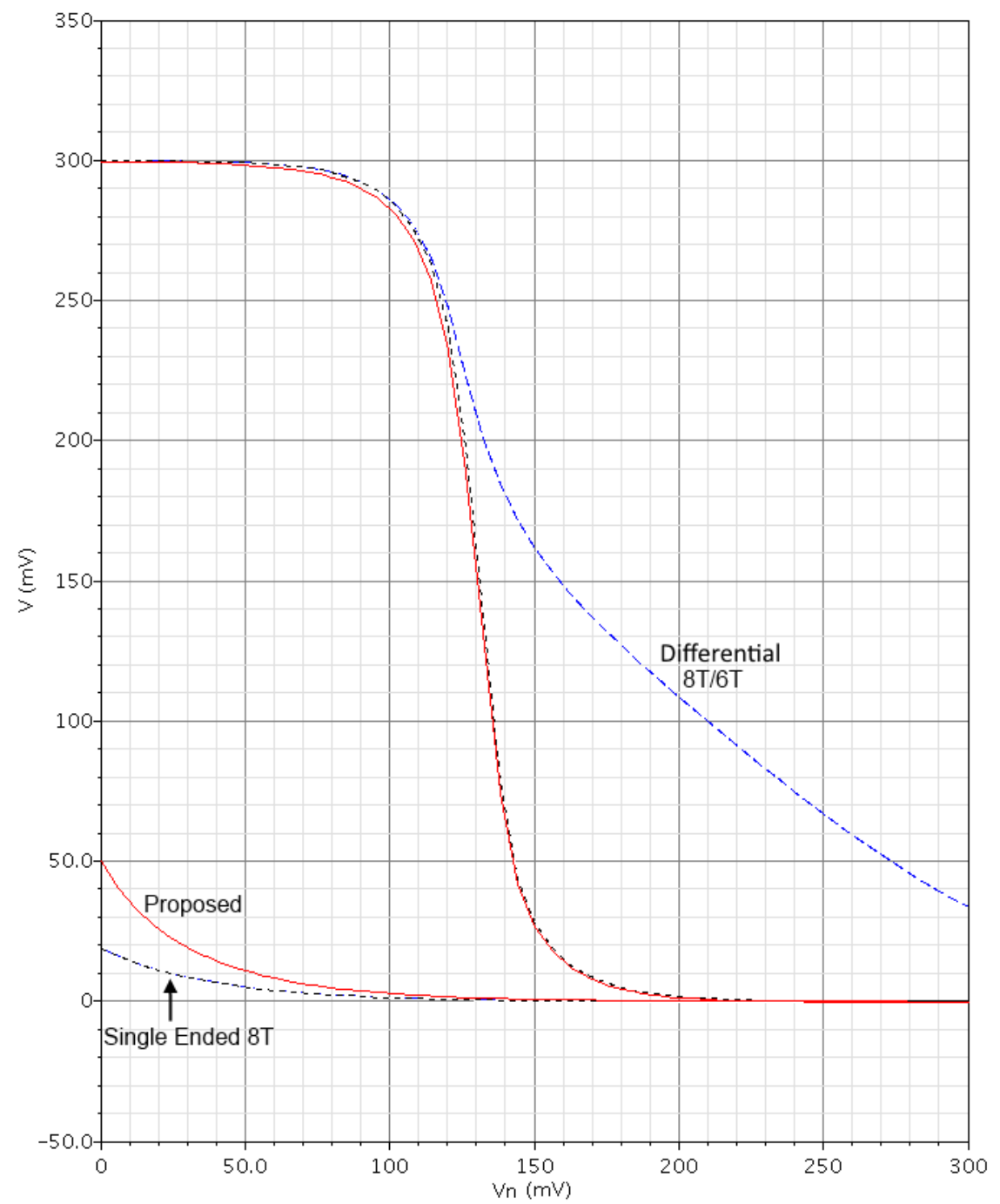

Figure 4.9: Comparison of the write SNM of the proposed design, the differential traditional $8 \mathrm{~T} /$ standard $6 \mathrm{~T}$ (they are same in this case), and single-ended $8 \mathrm{~T}$ write schemes. The $V_{D D}$ used in the simulation is $300 \mathrm{mV}$.

The write static noise margin (WSNM) is comparable to that of a conventional 8T (or 6T) single-ended write scheme. This is illustrated in Figure 4.9. Compared to a traditional differential write scheme, where the write SNM is $147.1 \mathrm{mV}$, the write $\mathrm{SNM}$ is $27.2 \mathrm{mV}$ worse $(119 \mathrm{mV})$ because only the voltage at one node is being pulled 
down. In a differential write scheme, one node is pulled up while the other is pulled down. While pulling a node down is what actually completes the write, pulling the other node up increases the SNM. Rarely is the write SNM the bottleneck of a design however.

The proposed architecture may not seem to support bit-interleaving due to the horizontal bit-lines, but it is actually the traditional word structure, where all bits in a word are adjacent, that is not supported. It is not possible to concurrently write into a cell that is directly adjacent (i.e. any cell that is within the same cell block). While this also makes it impossible to arbitrarily write a bit in any position, it does provide a solution to half-select disturb and, thus, supports a bit-interleaving structure. This bit-interleaving structure provides the ability to concurrently write into any one cell in each block in the same row. It also puts a minimum limit on the number of cell blocks in an SRAM block. There must be at least $x$ cell blocks in a row, where $x$ is the number of bits in a word, assuming the unit of address resolution is a word.

Since the word-lines connected to the access transistors of the cells are vertical, half-select disturb is not an issue for cells in the same row as they remain isolated. Instead, cells in the same column are susceptible to half-select disturb. This is shown in Figure 4.10. In this state, all the pull-down transistors connected to the local bit-line are OFF and a single access transistor is ON. The half-selected bit-cell ( $Q_{1}$ in Figure 4.10) is exposed to the disturbances in the local bit-line (LBL), resulting in a decrease in stability.

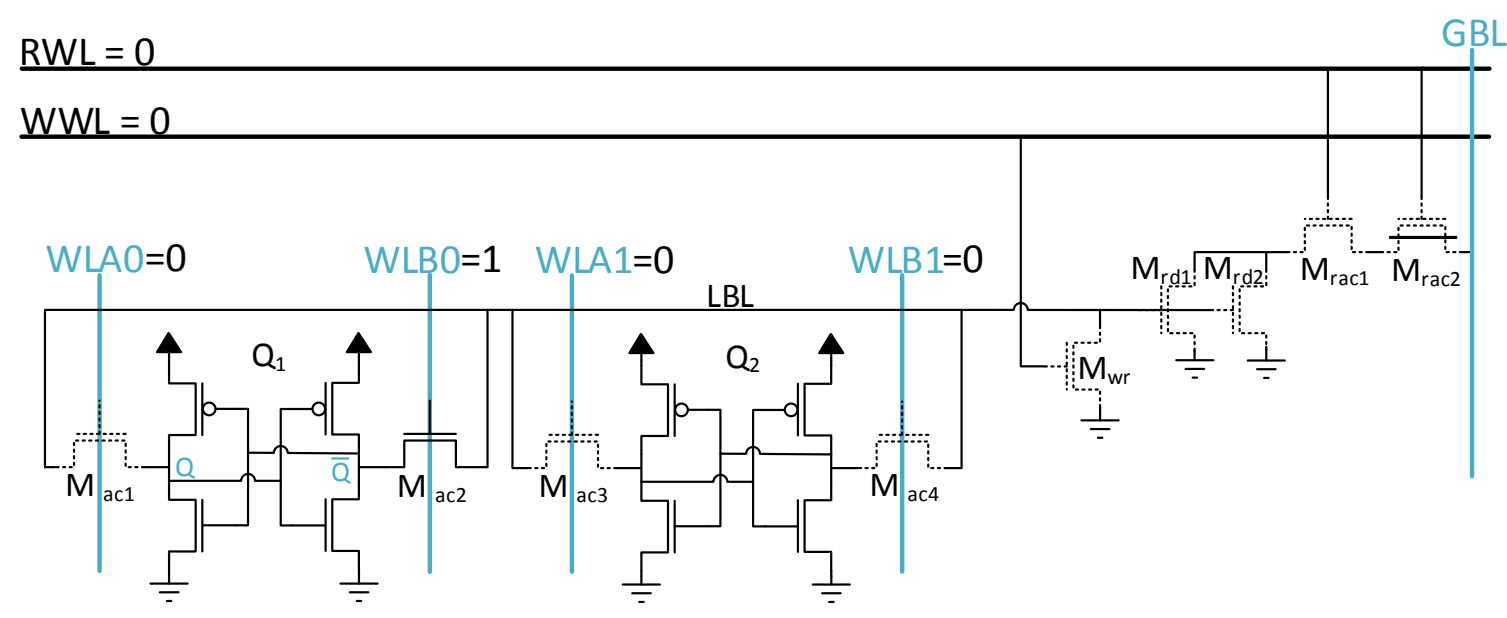

Figure 4.10: Half-select disturb in the proposed design. 
Arguably, column half-select disturb is a greater issue than row half-select disturb because, typically, there are more cells in a column than in a row (since the thincell layout of the standard 6T SRAM cell is longer horizontally than vertically). However, since the local bit-line is relatively short in contrast to conventional bitlines, the disturbance caused by other cells on the bit-line is comparatively very small. Additionally, the stability of the half-selected bit cells are the same as the read cell stability (i.e. read SNM), which is somewhat close to the hold cell stability (i.e. hold SNM).

\subsection{Read Operation}

Cell selection for the read operation is done by enabling the read word-line (RWL) for the row containing the cell and WLB (word-line B) for the column containing the cell. These steps turn on one access transistor for the selected cell and the read access transistors $\left(M_{\text {rac1-2 }}\right)$. The global bit-line is then conditionally discharged depending on the data stored in the selected cell. As discussed in the previous section, it is inconsequential whether WLB or WLA is used for selecting the column as long the same word-line is always selected for the read operation. In this thesis, WLB will be used for reads.

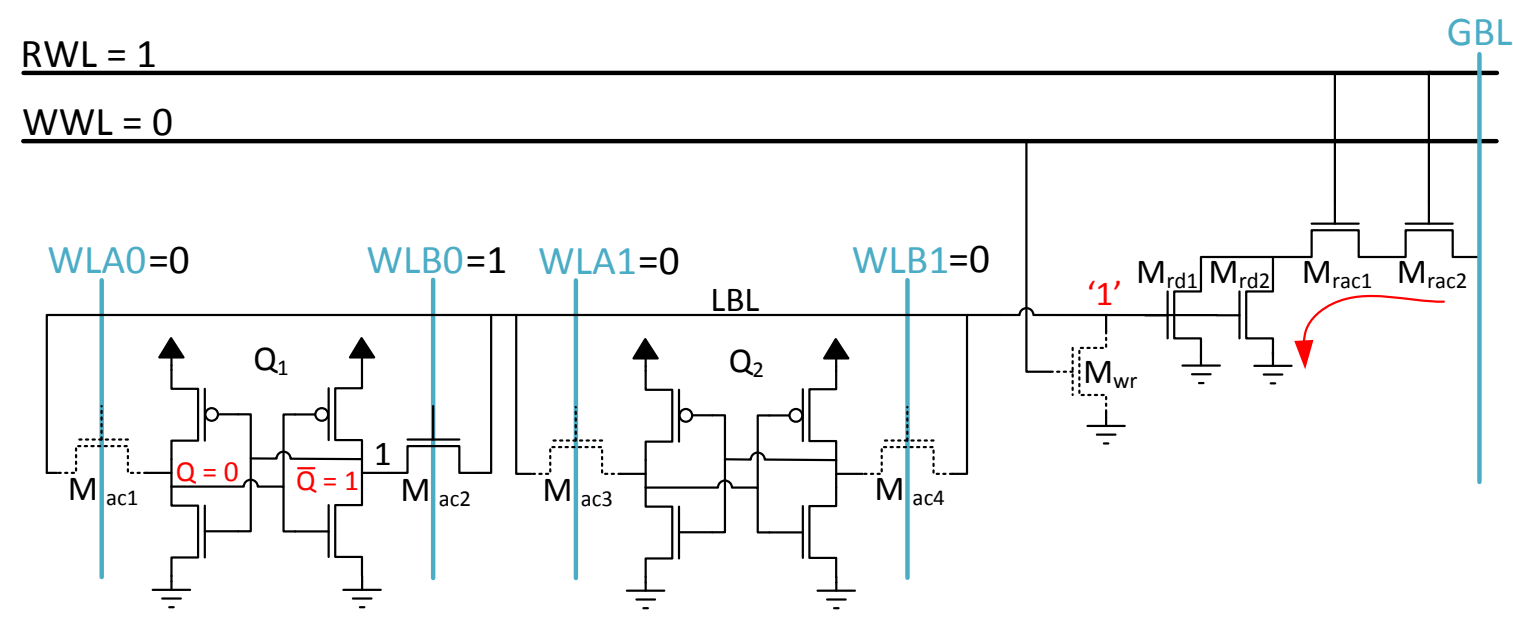

Figure 4.11: Read operation of the proposed design when reading a ' 1 '.

If the data stored in the cell at $\bar{Q}$ is a ' 1 ', the read decoupling transistors $\left(M_{r d 1}\right.$ 
and $M_{r d 2}$ ) are turned on. This results in the pre-charged global bit-line to discharge through the read access and decoupling transistors. A full-swing large signal sense amplifier is then used to read the resultant value. Figure 4.11 shows the read operation when the read data is a ' 1 '. The timing diagram when reading a ' 1 ' is shown in Figure 4.14. If the data stored in the cell at $\bar{Q}$ is a ' 0 ', the read decoupling transistors remain off, and the pre-charged global bit-line remains high. Figure 4.12 shows the read operation when the read data is a ' 0 '. The timing diagram when reading a ' 0 ' is shown in Figure 4.15.

When reading a ' 1 ', the data stored in the cell at $\bar{Q}$ is a ' 1 '. Thus, when the access transistor for the cell is enabled, the local bit-line obtains the value ' 1 '. However, since NMOS transistors are used for the access transistors (which are required for strong writes), it is not possible for the local bit-line to attain a voltage level of $V_{D D}$. The local bit-line charges close to $V_{D D}$, but not fully. As a result, the read decoupling transistors $\left(M_{r d 1}\right.$ and $\left.M_{r d 2}\right)$ are not fully $\mathrm{ON}$.

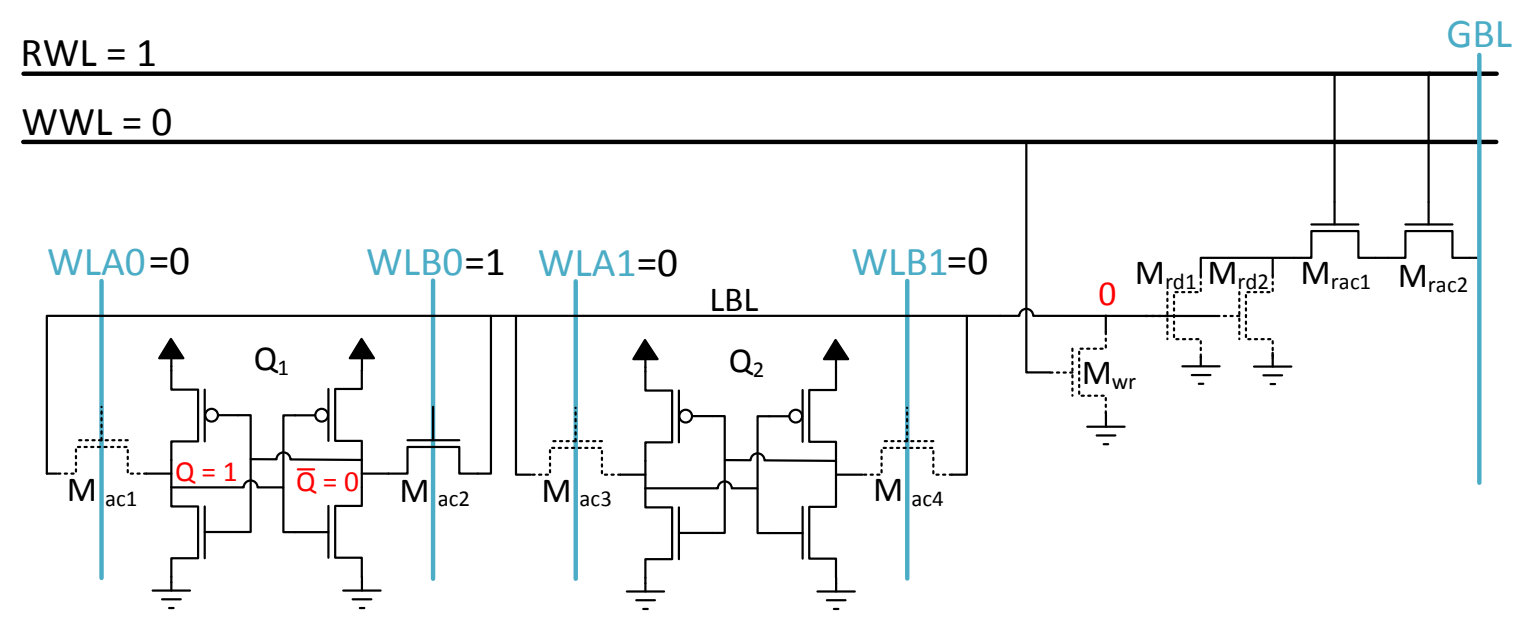

Figure 4.12: Read operation of the proposed design when reading a '0'.

A single read-decoupling transistor would slow down the read operation compared to the standard 8T cell. However, utilizing 2 minimum sized transistors in parallel further increases the current while keeping all transistors the same size to reduce process variations (as discussed in Section 2.8). Figure 4.13 shows the bit-line drainage current when a single minimum sized transistor, a single oversized transistor (2 times the minimum width), and a pair of minimum-size parallel stacked transistors (PTS 


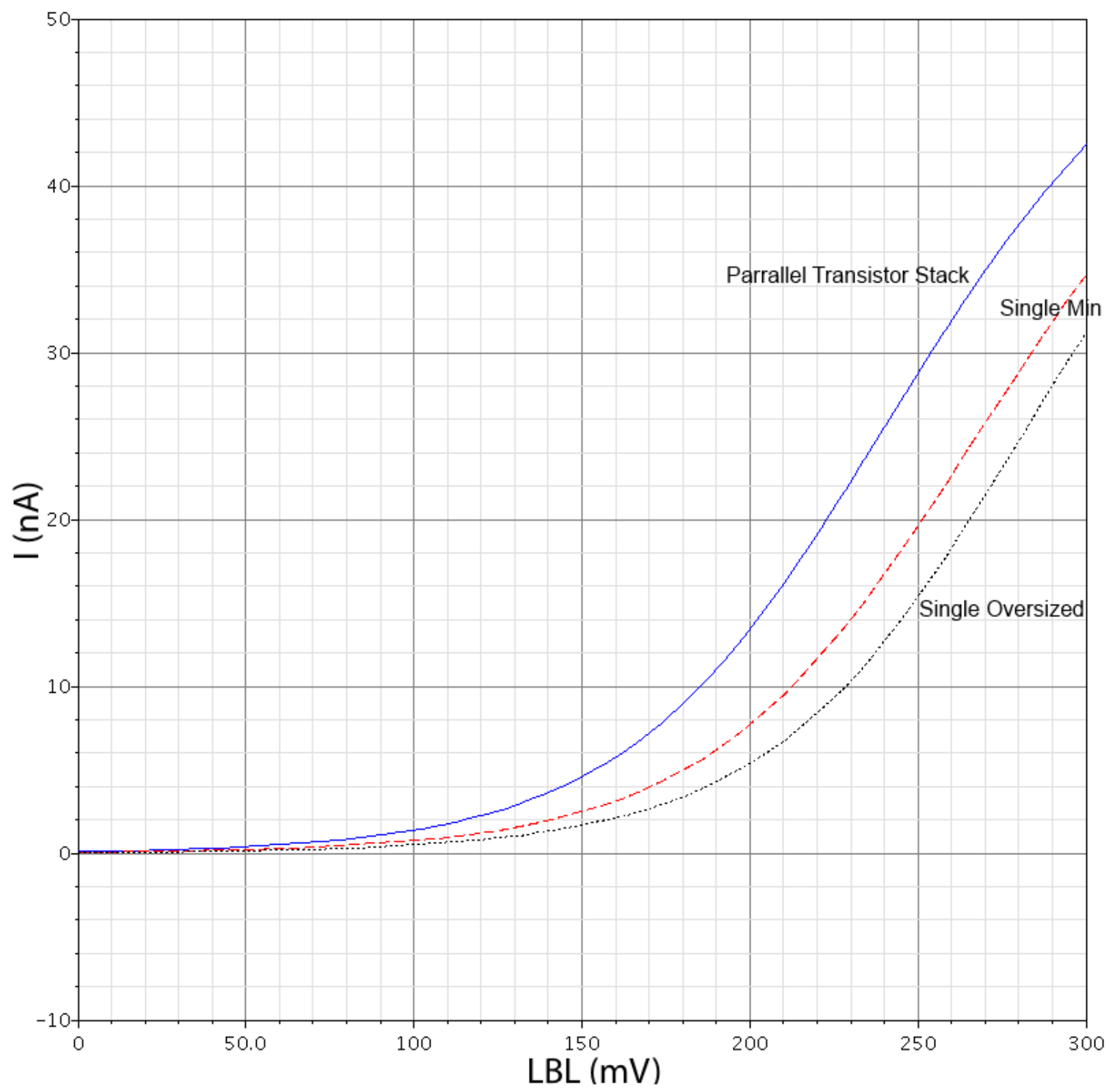

Figure 4.13: Bit-line drainage current when a single minimum-size transistor, a single oversized transistor (2 times the minimum width), and a pair of minimumsize parallel stacked transistors (PTS [14]) are used for read-decoupling. Simulation is performed for the FS corner. 

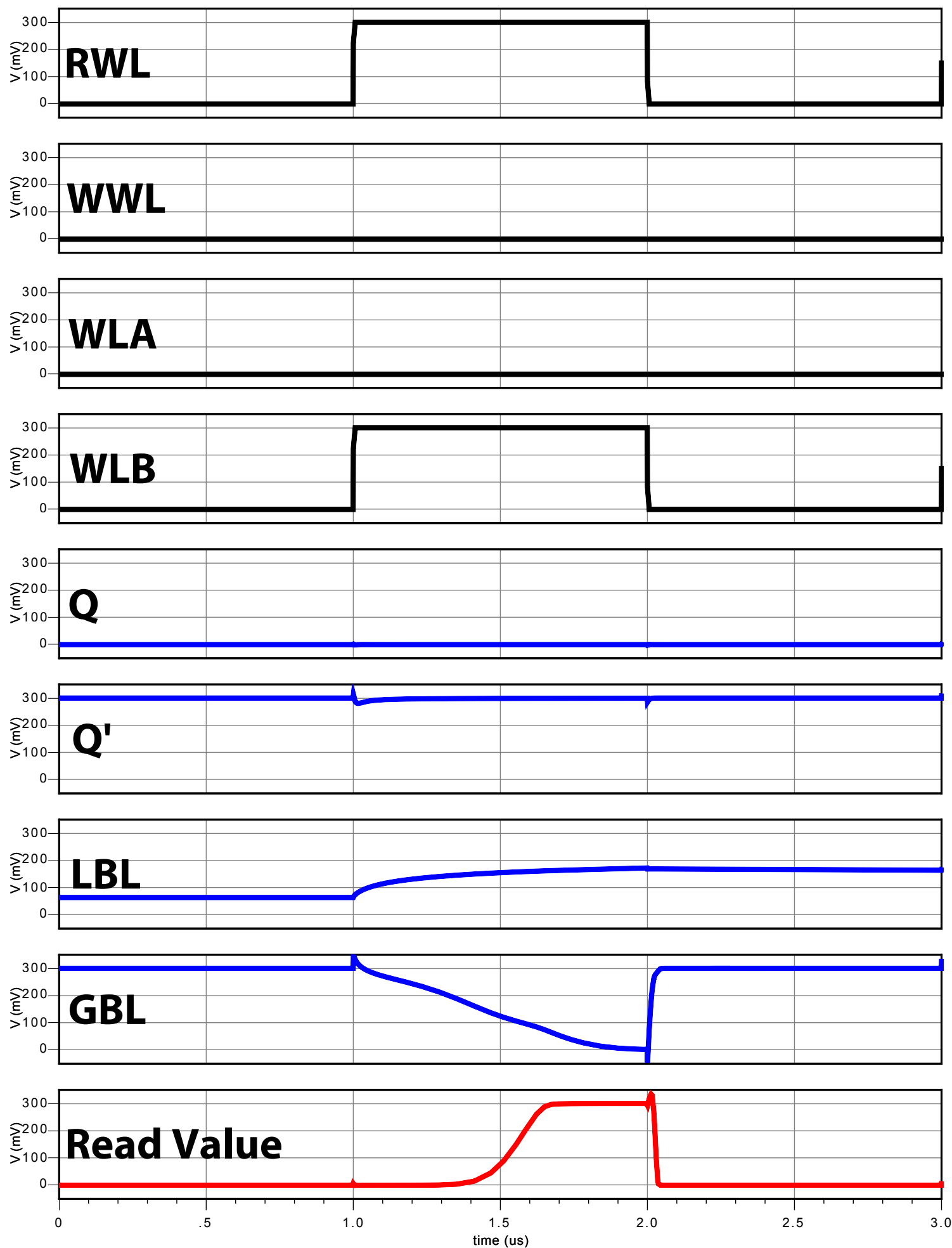

Figure 4.14: Timing diagram of the read operation when reading a ' 1 '. 

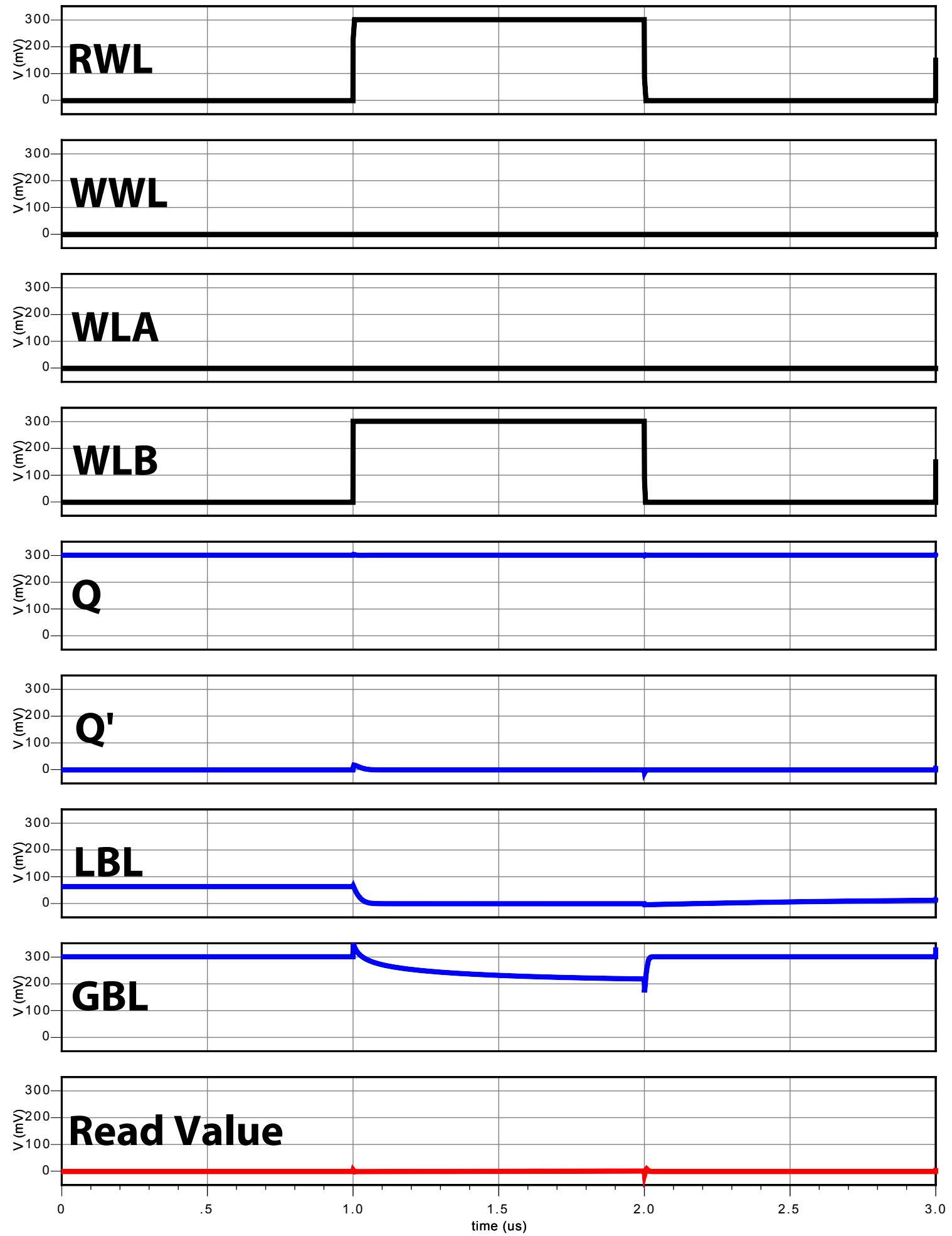

Figure 4.15: Timing diagram of the read operation when reading a ' 0 '. 
[14]) are used for read-decoupling. Using a larger width for the read decoupling transistor actually results in a lower current. This phenomenon is explained by the inverse narrow width effect.

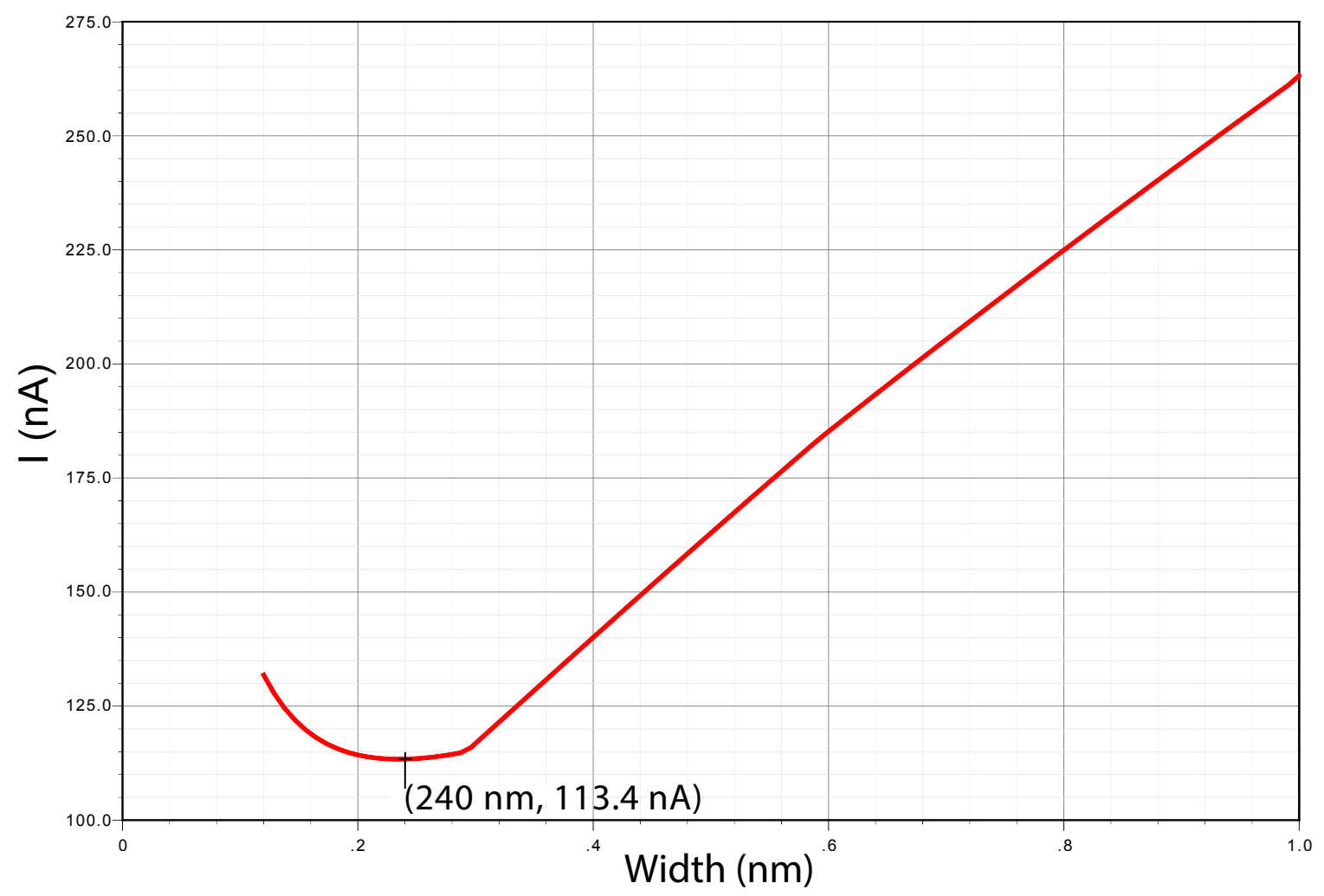

Figure 4.16: Drain current vs. width for an NMOS transistor. The inverse narrow width effect can be observed by comparing the drain current of a minimum width transistor with one twice the size (marked on the figure).

The inverse narrow width effect causes the threshold voltage, and consequently the drain current, to vary as the transistor width changes. This effect is caused by the fringing fields at the sharp corner in the shallow-trench isolation process that is used in the TSMC $65 \mathrm{~nm}$ process. The fringing field at this corner leads to the formation of inversion charges, making it easier to form a conduction channel at a lower voltage. As the transistor width decreases, the fringing capacitance contributes more to the overall electric field, causing the threshold voltage to lower [45], [46]. This effect can 


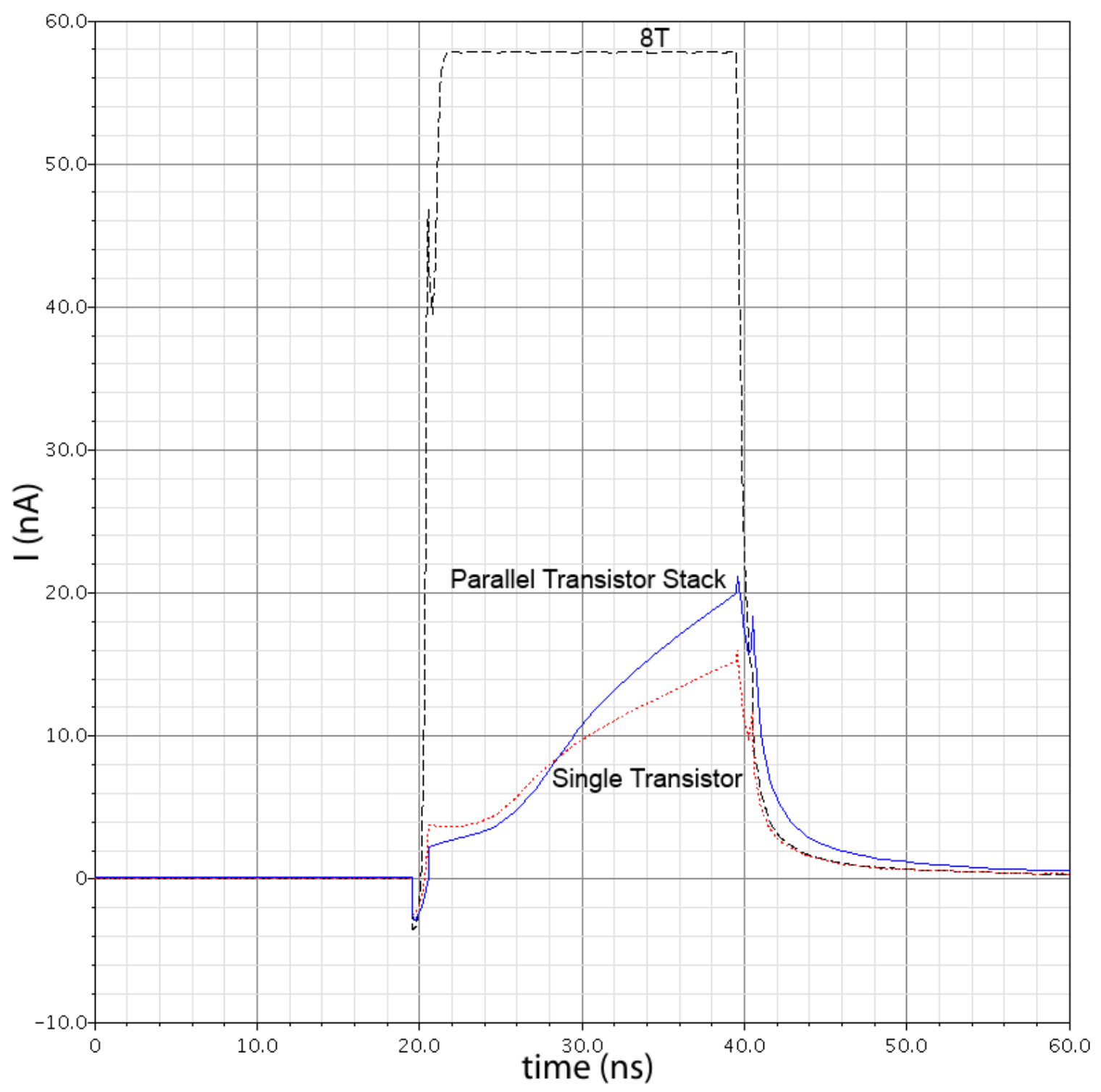

Figure 4.17: Comparison of the read current when a single minimum-size transistor, and a pair of minimum-size parallel stacked transistors (PTS) are used as read decoupling transistors when reading a ' 1 '. The read current of the traditional $8 \mathrm{~T}$ cell is included for comparison purposes. Simulation was performed for the FS corner.

be observed in Figure 4.16.

While parallel stacked read-decoupling transistors do increase the read current, it is important to remember that the read decoupling transistor is never fully ON. Consequently, the read current is never as strong as in the standard $8 \mathrm{~T}$ cell, in fact 


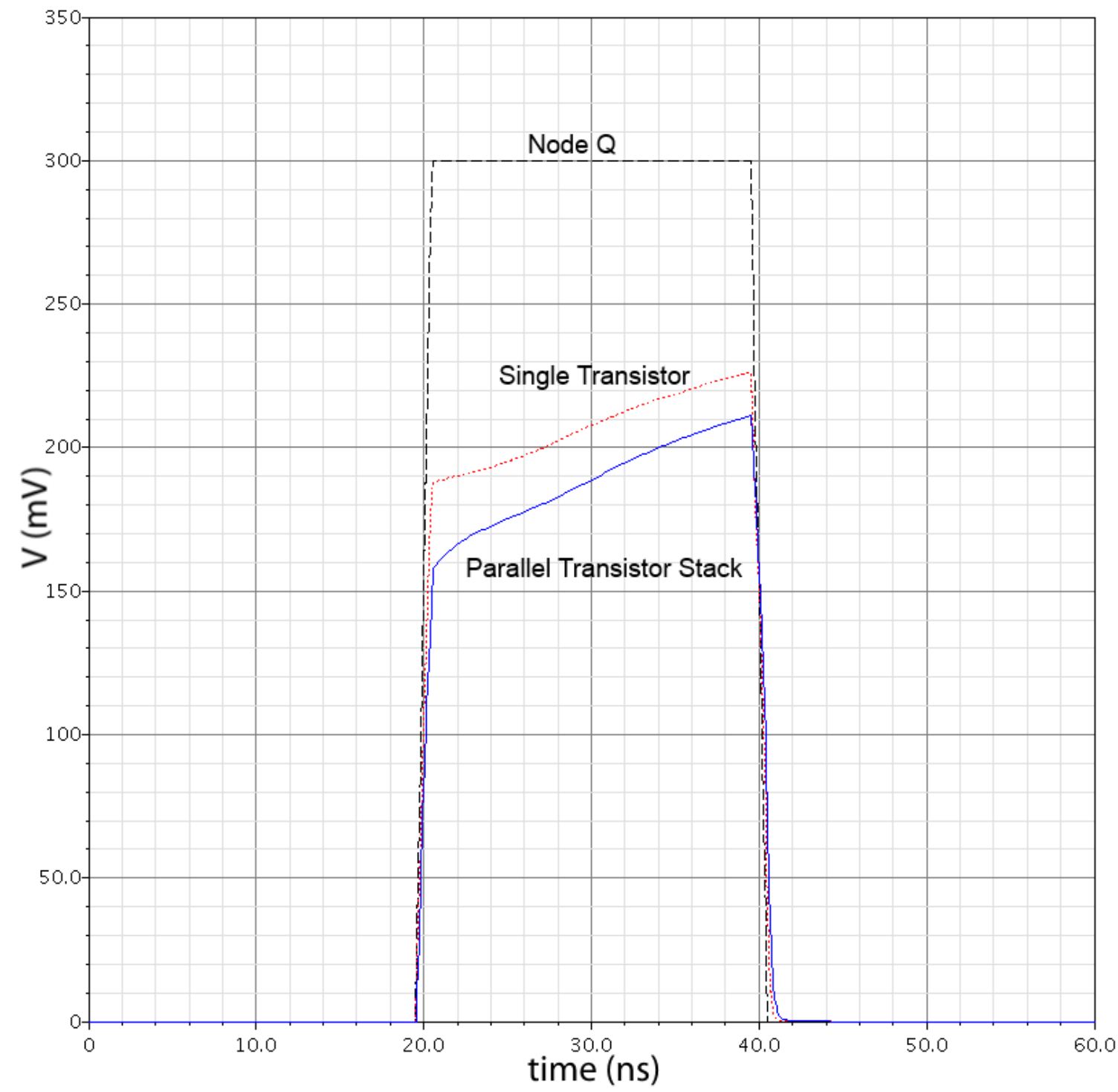

Figure 4.18: Comparison of the local bit-line voltage when a single minimum-size transistor, and a pair of minimum-size parallel stacked transistors (PTS) are used as read decoupling transistors when reading a ' 1 '. The voltage level of the data being read (Node Q) is included for comparison purposes. Simulation was performed for the FS corner.

it is many times worse. As a result, the time it takes to discharge the global bit-line during reads is decently longer. Figure 4.17 compares the read current when a single minimum-size transistor, and a pair of minimum-size parallel stacked transistors are used as read decoupling transistors when reading a ' 1 '. Figure 4.18 shows the local 
bit-line (LBL) voltage for both cases, as well as the node voltage of the data being read in the SRAM cell.

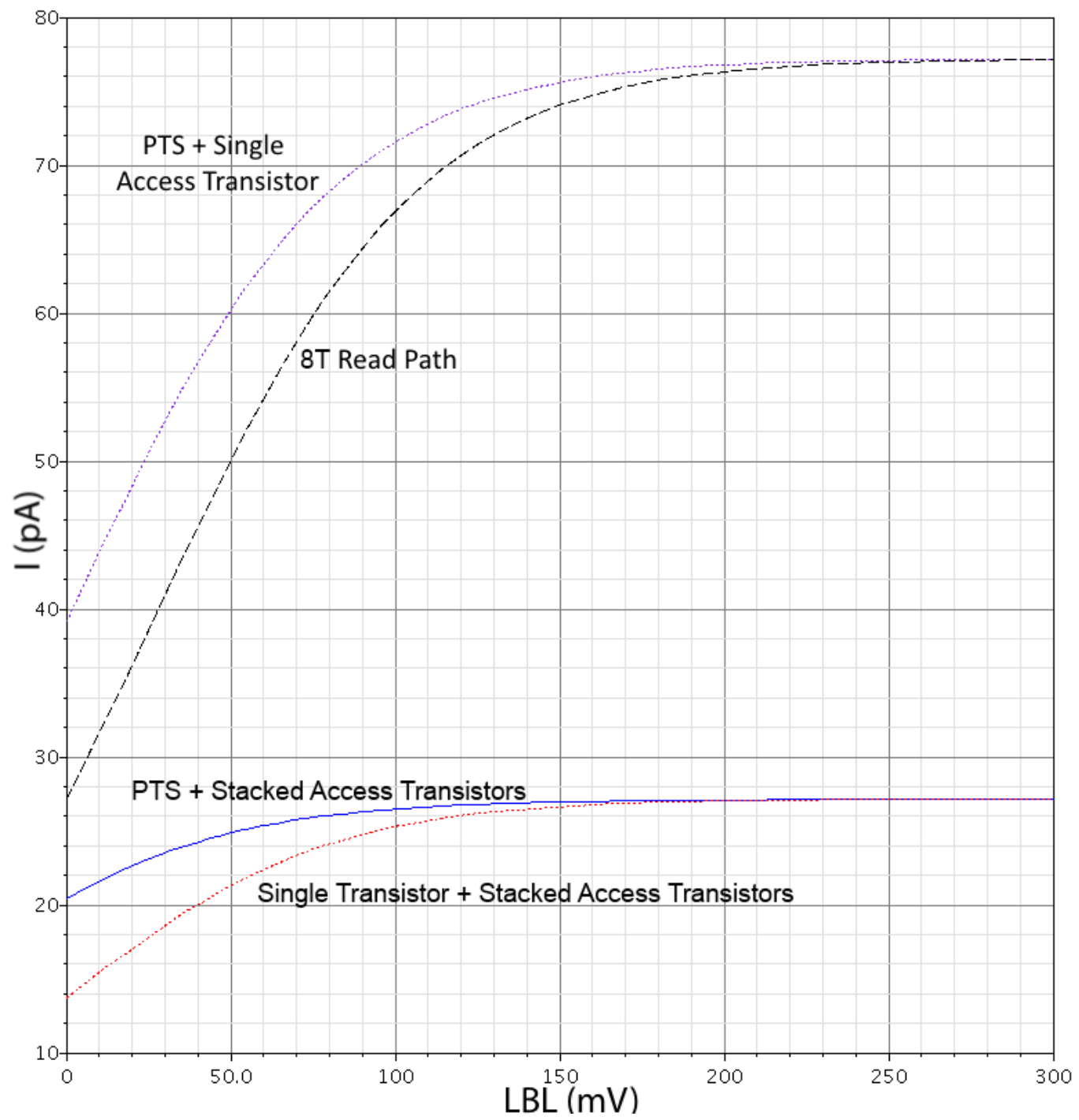

Figure 4.19: Comparison of the bit-line leakage for a combination of parallel read-decoupling transistors and stacked read-access transistors, a single readdecoupling transistor and stacked read-access transistors, and parallel readdecoupling transistors and a single read-access transistor. The bit-line leakage for the read path in the 8T SRAM cell is included for comparison. The simulation is performed for the FS corner.

It is important to note that the read operation is also susceptible to half-select disturb. The state of the cell block is the same as in Figure 4.10; all the pull-down transistors connected to the local bit-line are OFF and a single access transistor is 
ON. Again, the exposed bit-cell ( $Q_{1}$ in Figure 4.10) has decreased stability and is subject to the disturbances in the local bit-line. However, this has an additional impact on the read operation. Since the value of LBL (the local bit-line) is set by $\bar{Q}$ in $Q_{1}$, this exposure affects the amount of global bit-line leakage. In the worst case, LBL will be set to a logic ' 1 ', enabling the read decoupling transistors-greatly increasing the leakage.

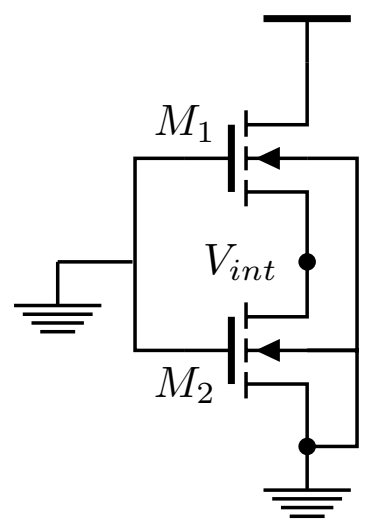

Figure 4.20: NMOS transistors stacked in series.

To reduce the global bit-line leakage, the read access transistors are stacked in series. The leakage current through a stack of series-connected transistors is greatly reduced when more than one transistor in the stack if OFF as a result of the stacking effect and is smaller than the leakage in a single transistor. An NMOS transistor stack is illustrated in Figure 4.20. The sub-threshold (and leakage) current can be found by the following equations:

$$
\begin{aligned}
I_{\text {sub }} & =I_{0} e^{\frac{V_{G S}-V_{T H 0}-\eta V_{D S}+\gamma V_{B S}}{n V_{T}}}\left(1-e^{\frac{-V_{D S}}{V_{T}}}\right) \\
I_{0} & =\mu C_{O X} \frac{W}{L}(n-1) V_{T}^{2} \\
V_{T} & =\frac{k T}{q}
\end{aligned}
$$

where $V_{G S}, V_{D S}$, and $V_{B S}$ are the gate-to-source, drain-to-source, and bulk-tosource voltages, respectively, $V_{T H 0}$ is the zero biased threshold voltage, $\eta$ is the drain induced barrier lowering coefficient, $n$ is the sub-threshold swing coefficient, $V_{T}$ is the thermal voltage, $\gamma$ is the body effect coefficient, $\mu$ is the carrier mobility, $C_{O X}$ is the 
gate oxide capacitance per unit area, $W$ and $L$ denotes the channel width and length, $k$ is the Boltzmann constant, $T$ is the absolute temperature, and $q$ is the electrical charge of an electrons [47].

Equation 4.1 shows that doubling the channel length reduces the off-current by a factor of 2. However, due to the reverse short channel effect in modern deep submicron devices, the threshold voltage tends to decrease for longer channels, making leakage reduction less effective. Transistor stacks are a more promising approach for reducing leakage in these modern devices.

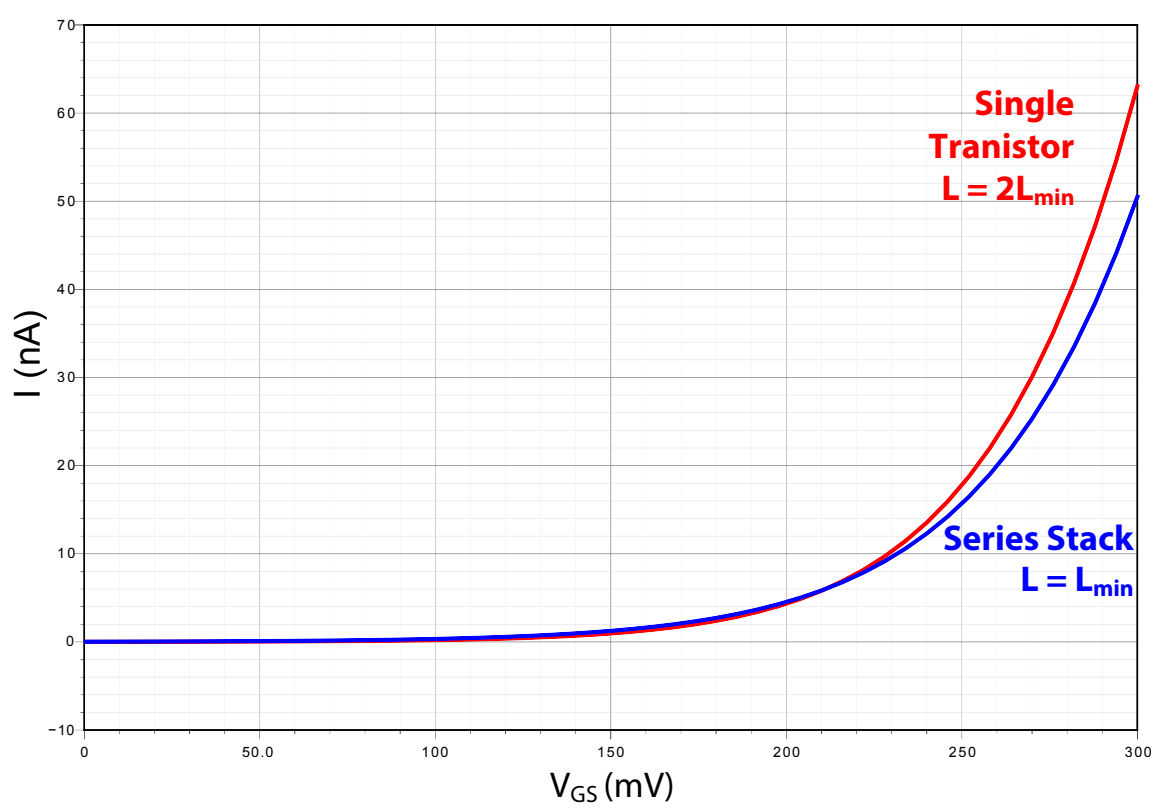

Figure 4.21: Comparison of the sub-threshold current between two minimum-length series stacked NMOS transistors and one NMOS transistor with a length of two times the minimum-length. $V_{D D}$ is $300 \mathrm{mV}$.

The intermediate voltage $\left(V_{i n t}\right)$ between two OFF stacked transistors is below $V_{D D}$, but remains positive due to a small drain current. Since the gates of the OFF NMOS transistors are connected to GND and $V_{i n t}$ is positive, the gate-to-source voltage of the transistor at the top the stack $\left(M_{1}\right.$ in Figure 4.20) will have a negative voltage of $-V_{\text {int }}$, reducing drain current. Similarly, the body-to-source voltage of $M_{1}$ is also $-V_{\text {int }}$, resulting in a larger body effect and, thus, an increase in threshold voltage compared to a single transistor. Furthermore, the drain-to-source voltage of $M_{1}$ is lower than the drain-to-source voltage of a single transistor [48]. Substituting the relevant voltages into Equation 4.1 for the stacked transistors and a single transistor 
results in

$$
\begin{aligned}
& I_{\text {sub }}=I_{0} e^{\frac{-V_{i n t}-V_{T H 0}+\eta\left(V_{D D}-V_{i n t}\right)-\gamma V_{i n t}}{n V_{T}}}\left(1-e^{\frac{-\left(V_{D D}-V_{i n t}\right)}{V_{T}}}\right), \text { and } \\
& I_{\text {sub }}=I_{0} e^{\frac{-V_{T H 0}+\eta V_{D D}}{n V_{T}}\left(1-e^{\frac{-V_{D D}}{V_{T}}}\right),}
\end{aligned}
$$

respectively. Since $V_{\text {int }}>>\left(V_{D D}\right)$, these equations show that the series stacked transistor will have lower sub-threshold current [47]. This can be further observed in Figure 4.21.

Using series stacked transistors for the access transistors allows for the number of cell blocks connected to the global bit-line to be comparable to the number of bit-cells connected to the read bit-line in the standard $8 \mathrm{~T}$ architecture. In the worst case corner, i.e. FS (Fast NMOS, slow PMOS), the preeminent bit-line leakage is less than the paramount bit-line leakage in the standard 8T architecture, as shown in Figure 4.19. The FS corner is the worst case corner because the read access and decoupling transistors are NMOS and the pull-up transistors are PMOS. Stacked read-access transistors reduces the leakage current by close to 3 times. Utilizing parallel stacked read-decoupling transistors in conjunction with the stacked readaccess transistors does increase the leakage slightly when they are OFF, but the increase in ON current is, arguably, worth the tradeoff depending on the design goal.

The horizontal local bit-lines do not only help with the write operation, they also support the read operation. Compared to the long global bit-lines, the short local bitlines have the advantage of having a lower cumulative bit-line leakage due to a drastic reduction in the number of cells connected to it. This is especially advantageous in sub-threshold operation where the current of hundreds of unselected cells can greatly surpass the on current of a selected cell. This has a positive effect on the read static noise margin.

The read static noise margin (RSNM) of the proposed cell is relatively comparable to that of the traditional 8T SRAM cell, and much better than the standard 6T SRAM cell, in ideal conditions, as shown in Figure 4.22. Introducing PVT variations into the scenario results in a slightly worse read SNM when compared to the standard 8T SRAM cell. Due to the read operation not being isolated from the local bit-line, unlike the 8T SRAM cell, the cell is still subjected to bit-line charge sharing with the other cells connected to the same bit-line. As a result, the stability of the cell suffers 


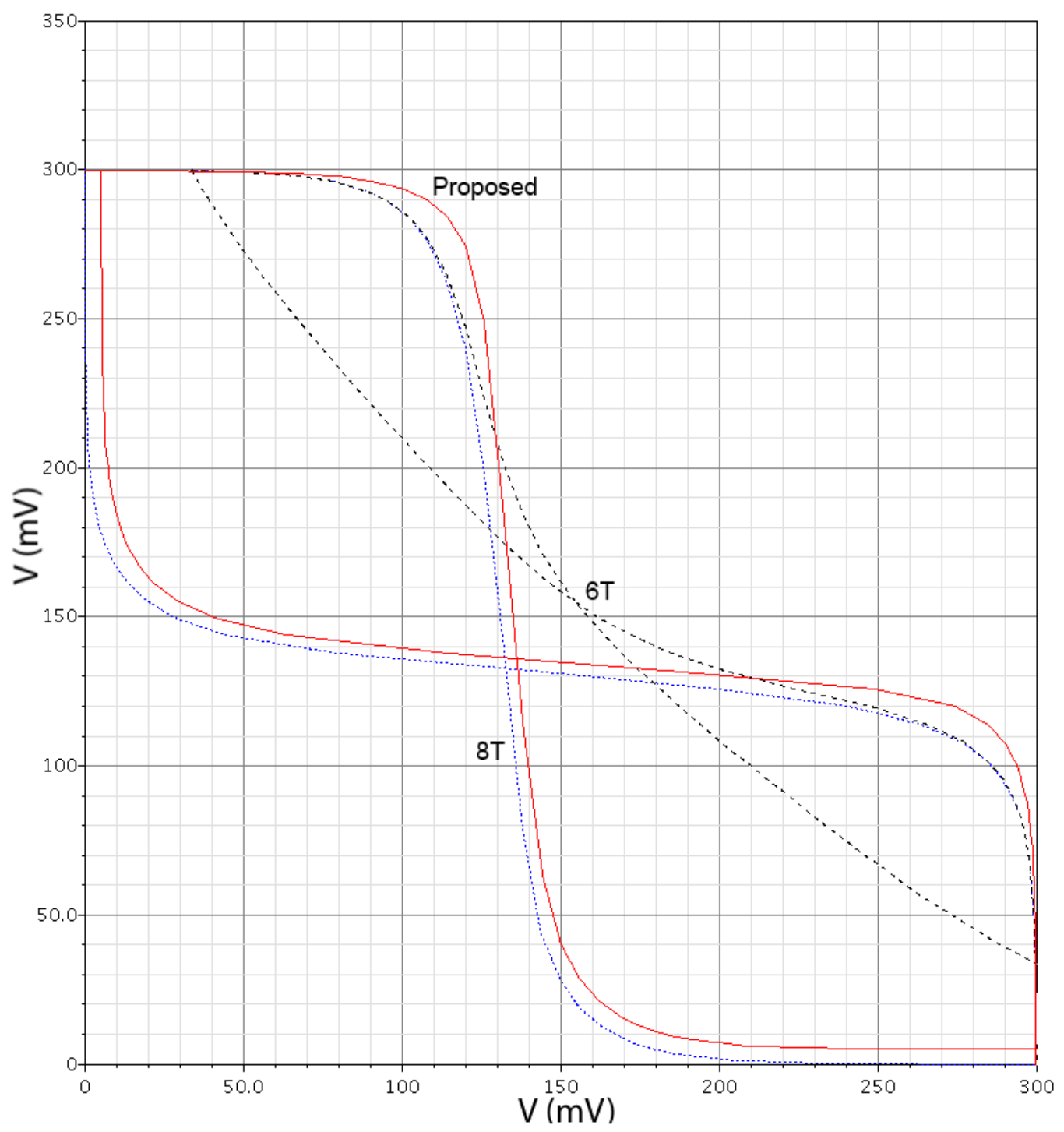

Figure 4.22: Comparison of the read SNM of the proposed design, the traditional $8 \mathrm{~T}$, and standard $6 \mathrm{~T}$ SRAM cells. The $V_{D D}$ used in the simulation is $300 \mathrm{mV}$.

slightly. Nevertheless, the comparatively short local bit-lines prevent the read SNM from being as abysmally small as in the standard 6T SRAM cell. In terms of cell stability, the read SNM is the weakest part of this design.

It is interesting to note that the proposed architecture is single-ended. However, horizontal bit-lines allows it to go a step further by only having one large capacitance global bit-line per cell block. That is, there is only one global bit-line per $x$ bits, where $x$ is the number of bits in each cell block. This decreases the dynamic power consumption by greatly reducing the number of bit-lines to pre-charge and discharge. 
Bit-line leakage is also significantly reduced since there are a limited amount of bitlines.

\subsection{Hold Operation}

The hold, or idle, operation is achieved by holding all word-lines low, which disables the access transistors. This leaves the local bit-line (LBL) floating. While global bit-lines may be disturbed, they are decoupled from the storage cells. Thus, the data remains unaffected. Figure 4.24 shows the hold static noise margin (HSNM). Since the storage cell is exactly the same as in the standard 6T and traditional 8T SRAM cells, the hold SNM is roughly the same in all cases.

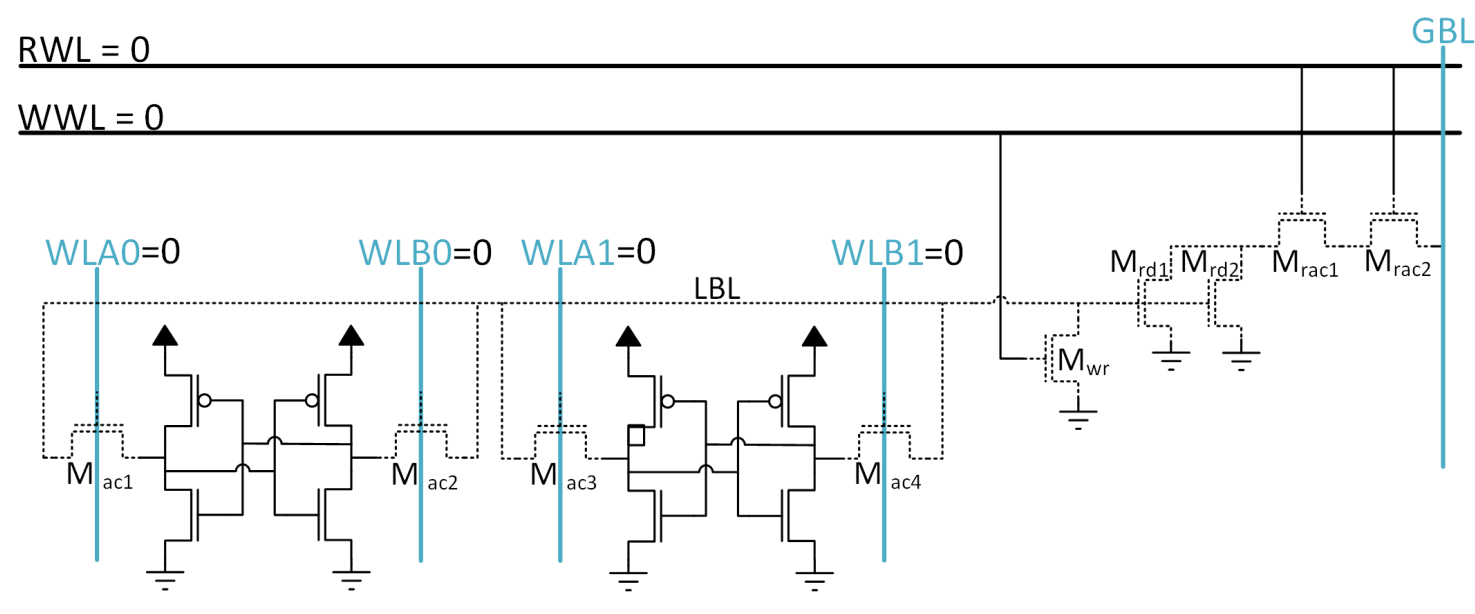

Figure 4.23: Idle operation of the proposed design.

\subsection{Cell Layout}

Since the proposed SRAM cell is a modification of the standard 6T SRAM cell, its layout is based on the $6 \mathrm{~T}$ thin-cell layout. The layout for the SRAM cell is illustrated in Figure 4.25. All transistors in the layout are minimum sized, creating a diffusionnotch-free layout. The thin-cell layout keeps the long global bit-lines, WLA, and WLB as short as possible to reduce their capacitances. This comes at the cost of a longer 


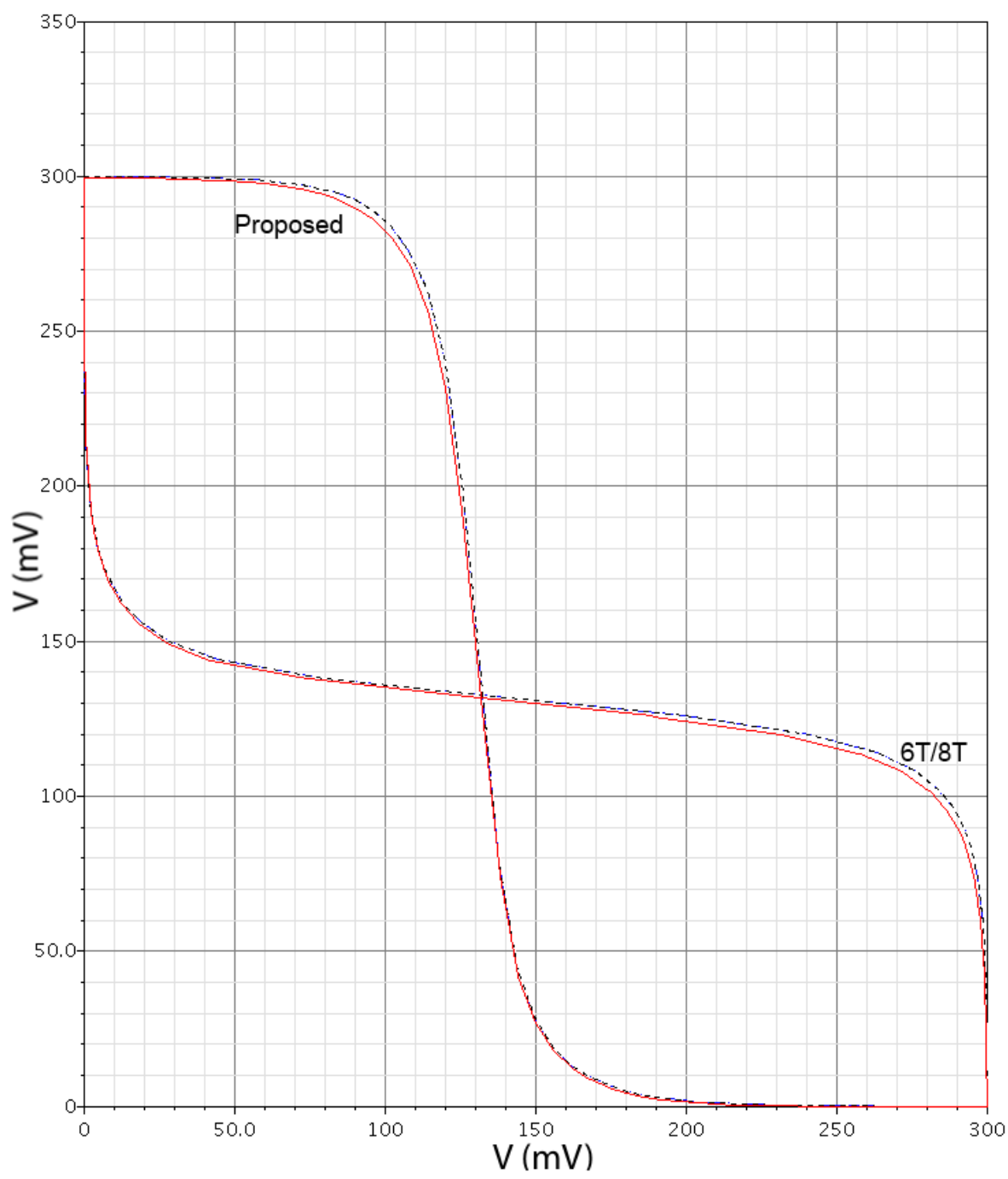

Figure 4.24: Comparison of the hold SNM of the proposed design, the traditional $8 \mathrm{~T}$, and standard $6 \mathrm{~T}$ SRAM cells for a $V_{D D}$ of $300 \mathrm{mV}$.

local bit-line, write word-line, and read word-line, but they are relatively small by comparison. It would be ideal to keep the local bit-line as short as possible to improve the read and half-select static noise margins, but this would result in increased process variations and/or increased delay due to the higher capacitance global bit-lines. The area of the SRAM cell is roughly the same as the thin-cell layout at $2.91 \mathrm{\mu m}^{2}$.

The layout for the overhead circuitry required for the cell block is depicted in Figure 4.26. The transistors are all aligned in the same direction to reduce process variations. The total area of this overhead section is around $2.14 \mathrm{\mu m}^{2}$. 


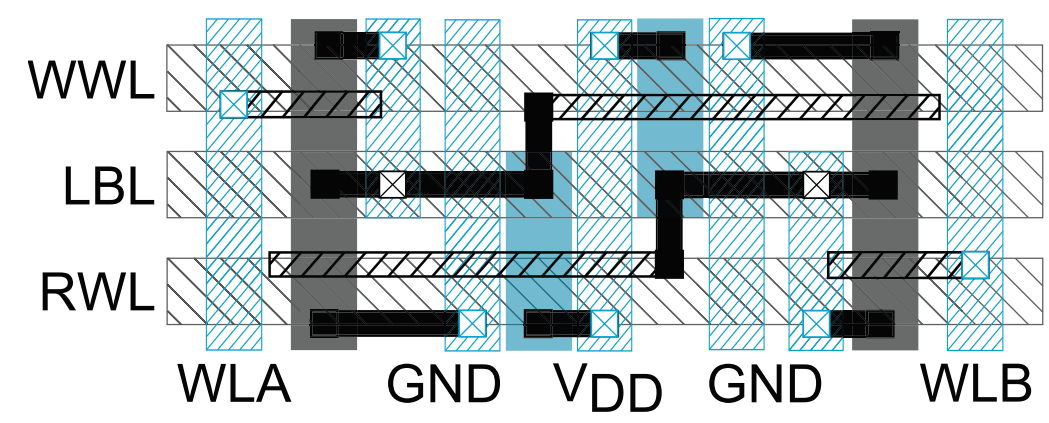

Figure 4.25: Layout of the proposed bit cell. The layout is based on the layout of the $6 \mathrm{~T}$ thin-cell.

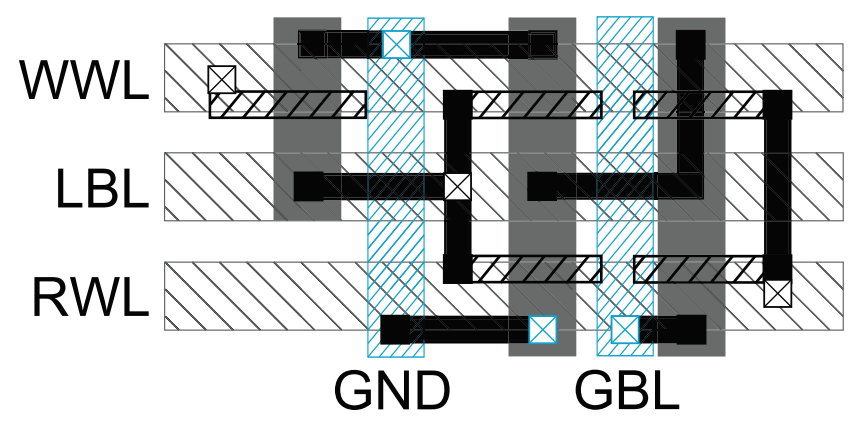

Figure 4.26: Layout for the overhead circuitry required for the proposed cell block, including read and write decoupling transistors, and read access transistors.

The combined layout can be seen in Figure 4.27. The overhead block circuitry is placed in the middle of the cell block with the SRAM cells placed to either side. The local bit-line (LBL) stops at the end of the cell block, unlike the write word-lines (WWL), and read word-line (RWL) that run for the entire row. All vertical wordlines and bit-lines are routed in metal 2, and all horizontal word-lines and bit-lines are routed in metal 3. The total area of the 8-cell configuration is $25.42 \mu^{2}$. This results in an average area of $3.18 \mathrm{\mu m}^{2}$ per bit cell or around 1.1 times the area of the $6 \mathrm{~T}$ cell. Thus, it can be said that this design has an average area of $6.6 \mathrm{~T}$. This is very close to the area of the standard $6 \mathrm{~T}$ cell, but with much better read SNM. 


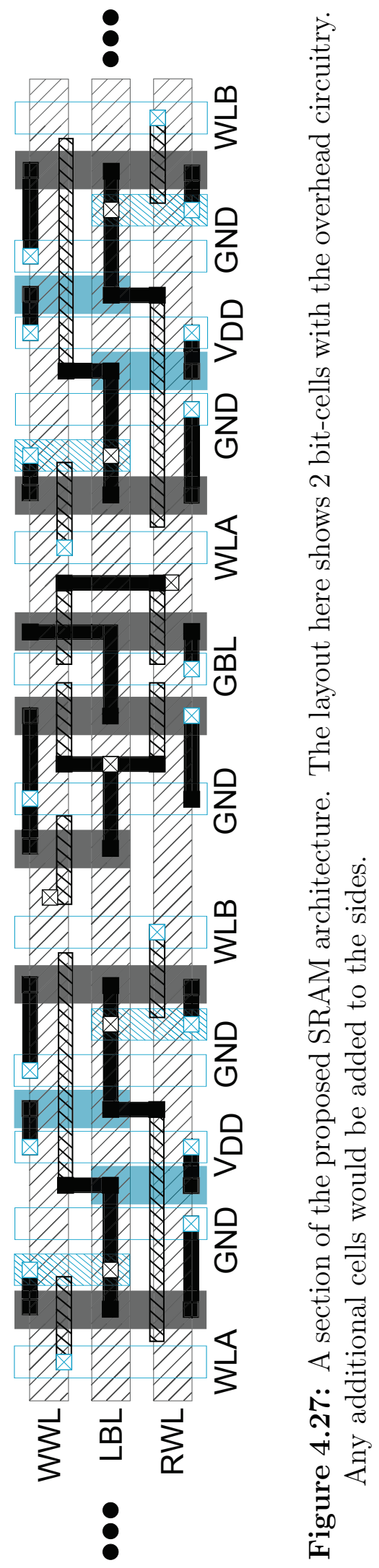




\section{Chapter 5}

\section{Static Noise Margin Evaluations}

To properly asses the proposed SRAM cell, the stability of the cell must be evaluated under all conditions, including process and temperature variations. Schematic simulations were done using Cadence Spectre (a SPICE class circuit simulator) using the TSMC $65 \mathrm{~nm}$ Mixed Signal RF salicide Low-K IMD process configured for 1.2 V supply voltage, which utilizes a BSIM4 (V4.5) model. The minimum length for the process is $60 \mathrm{~nm}$, the minimum width is $120 \mathrm{~nm}$, and the threshold voltage for a minimum sized NMOS transistor is $547.6 \mathrm{mV}$.

Monte Carlo simulations for the TSMC $65 \mathrm{~nm}$ Low Power process show that, at 11 bits in each cell block and a $V_{D D}$ of $300 \mathrm{mV}$, the read current of a single bit cell is always greater than the cumulative bit-line leakage caused by the unselected devices in the same block. To keep decoding simple, it is ideal for a block to contain a number of bits that is a power of 2. Thus, for the TSMC $65 \mathrm{~nm}$ LP process, 8 bits per block is the quintessential choice to minimize area. However, to explore how the number of bits in a cell block affects stability, cell blocks with configurations of 1 bit, 2 bits, 4 bits, and 8 bits will all be tested. The schematics of these configurations are shown in Figure 4.2, Figure 4.3, Figure 4.4, and Figure 4.5.

\subsection{Process Variations}

The compared points are plotted with a tolerance level of $\mu-5 \sigma$ to study the worst case scenarios, where $\mu$ is the arithmetic mean and $\sigma$ is the standard deviation. For normally distributed data, five standard deviations from the mean $(\mu \pm 5 \sigma)$ accounts for $99.9999426697 \%$ of all values. That is, only 1 out of 1744278 manufactured dies will be outside the range of $\mu \pm 5 \sigma$ [49]. Only the $\mu-5 \sigma$ values are reported 
because the smallest SNM is a concern, not the largest. The histograms the estimated probably density functions are related to, and the values of the simulation results can be found in Appendix A.

\subsubsection{Hold Operation}

As Figure 5.1 shows, there is very little change in the hold static noise margin as the number of bits in each cell block increases. There is only a 1 to $2 \mathrm{mV}$ difference in hold SNM between the 8-cells and 1-cell configuration. The hold SNM is relatively independent from the number of bits because there are two transistors (the access transistors) in series between every two bits that minimizes how each storage node affects each other. It is consistent, however, that as the number of bits increase, there is a minor increase in hold SNM. While this difference is within a few millivolts, at a $V_{D D}$ of $200 \mathrm{mV}$ there is a $33 \%$ improvement in the hold SNM when utilizing the 8-cell configuration over the 1-cell. This small correlation can be attributed to the slight increase in the capacitance of the local bit-line as the number of bits increase.

There is very little difference between the hold SNM for the traditional 8T, standard 6T, and proposed design, but as $V_{D D}$ drops, the difference becomes more apparent. At a $V_{D D}$ of $200 \mathrm{mV}$, the $8 \mathrm{~T}$ and $6 \mathrm{~T}$ cells have a $25 \%$ improvement in hold SNM compared to the 8-cells block configuration. The decrease in hold SNM in the proposed design is due to the floating local bit-line. In the $8 \mathrm{~T}$ and $6 \mathrm{~T}$ cells, the bitlines are pre-charged to logic ' 1 ', so the vast majority of the leakage will be through the access transistor connected to the node holding ' 0 '. In the proposed architecture, the voltage of the local bit-line can vary due to the leakage on the bit-line. Thus, the leakage through the access transistors can be higher than in the $8 \mathrm{~T}$ and $6 \mathrm{~T}$ cases.

The density functions of the traditional $8 \mathrm{~T}$ cell, standard $6 \mathrm{~T}$ cell, and the proposed architecture for the hold operation are all left-skewed normal distributions with very similar proportions. This can be verified by comparing the density functions in Figure 5.2. Note that since the density functions for all block configurations are very similar, only the 8 block configuration density function is included. 


\section{Hold SNM}

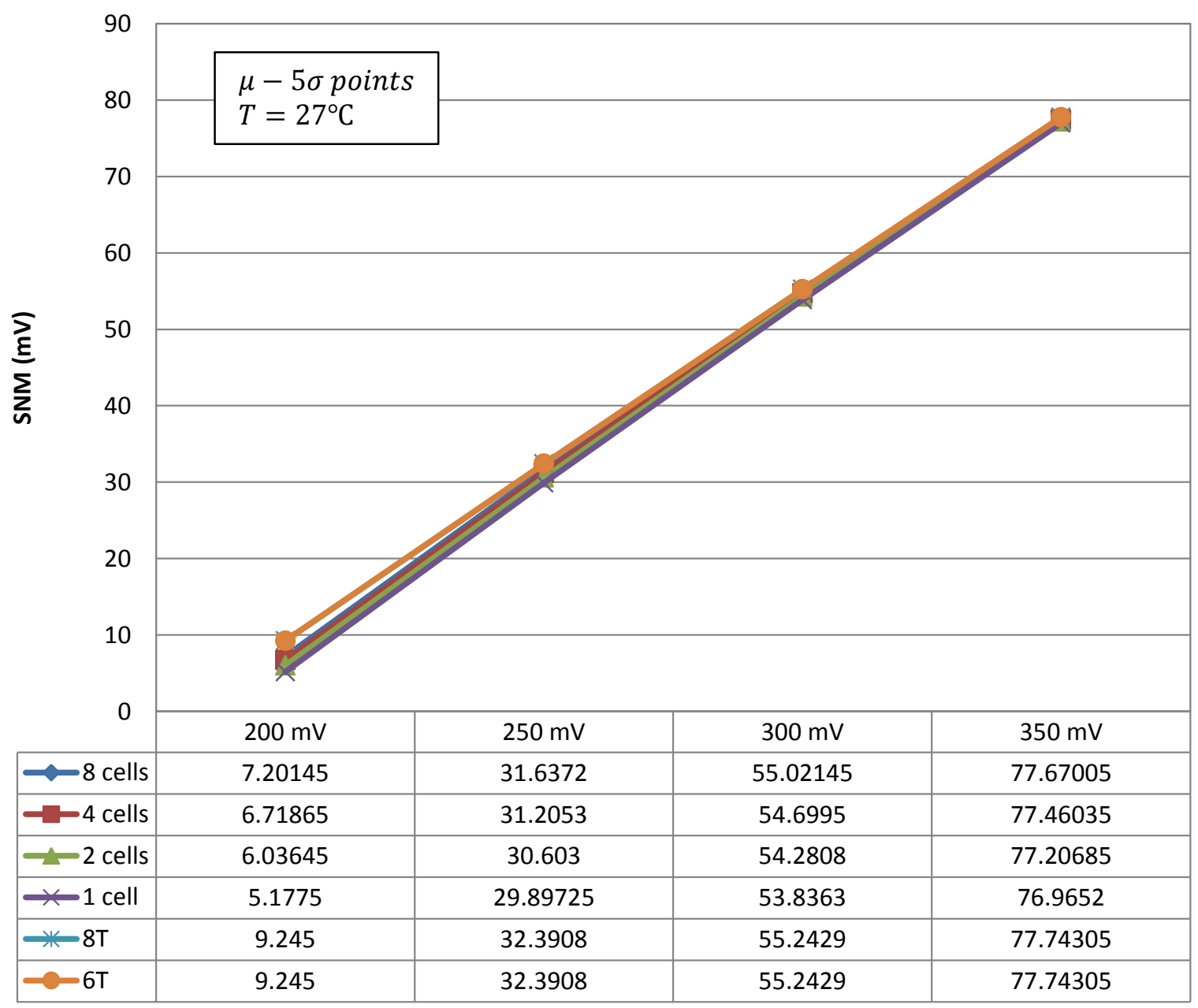

Figure 5.1: Simulation results for Hold SNM vs. $V_{D D}$ for the different block configurations proposed, the traditional $8 \mathrm{~T}$ cell, and the standard $6 \mathrm{~T}$ cell. The plotted points are $\mu-5 \sigma$. The points in the table are in $\mathrm{mV}$.

\subsubsection{Write Operation}

Like the hold operation, there is very little correlation between write static noise margin and the number of bits inside each cell block. There is at most $1.5 \mathrm{mV}$ difference between the 8-cell configuration and 1-cell configuration at a $V_{D D}$ of 200 $\mathrm{mV}$. This relative consistency can be explained by the write decoupling transistor keeping the local bit-line low in all cases.

Nevertheless, there is a slight, but noticeable, decrease in write SNM as more bits 


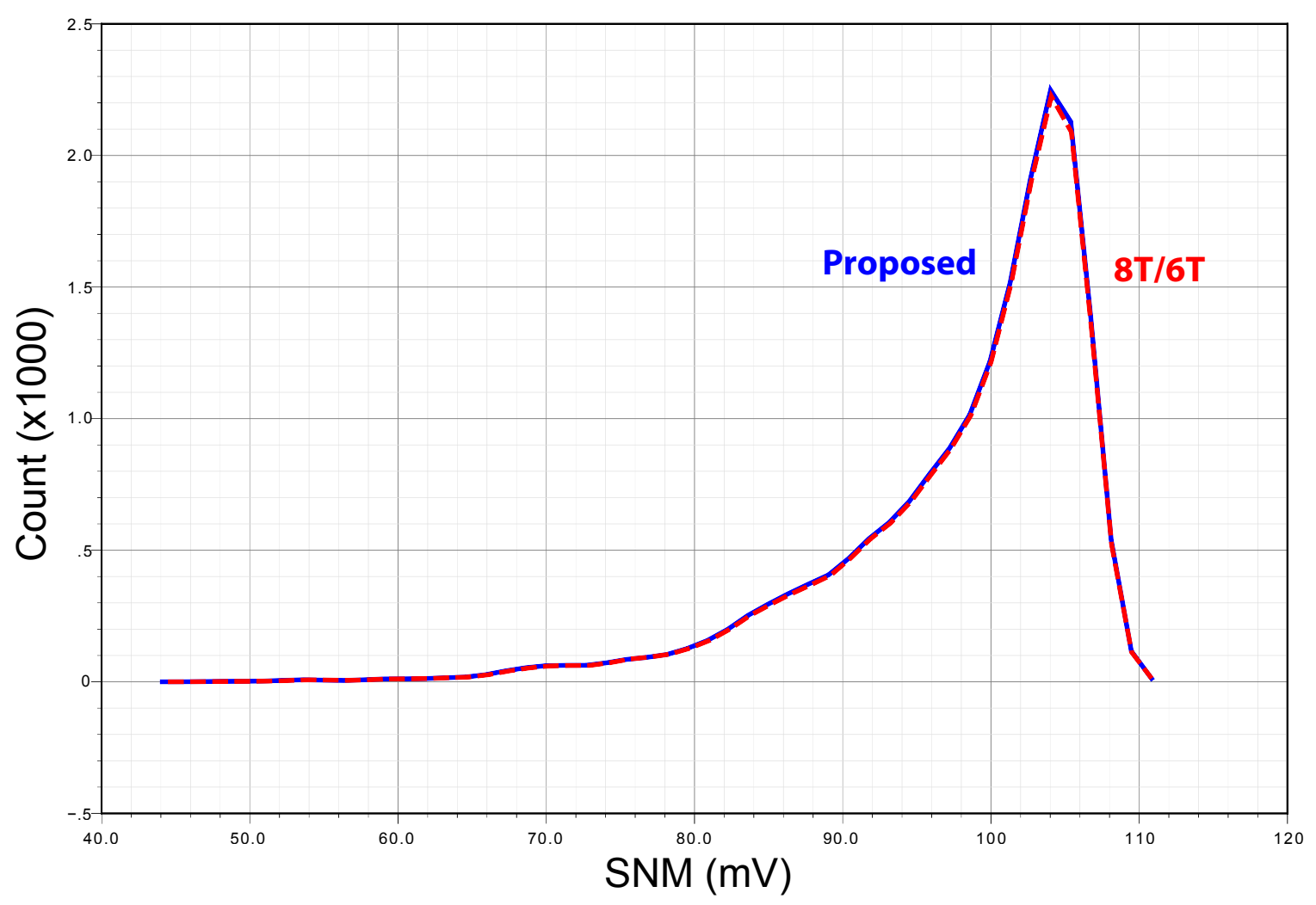

Figure 5.2: Estimated probably density function of the hold operation for the 8-cell block configuration and $8 \mathrm{~T} / 6 \mathrm{~T}$ SRAM cells (they are the exact same). $V_{D D}$ is $300 \mathrm{mV}$ and the temperature is $27^{\circ} \mathrm{C}$. Only the 8-cell configuration is included because all configurations have very similar distributions. This is similarly true for all sub-threshold operating voltages. Refer to Figure A.1, Figure A.2, and Figure A.3 for the histograms of the 8-cell block configuration, 8T SRAM cell, and 6T SRAM cell, respectively.

are added to each cell block. This is the result of enabling one access transistor, which exposes the storage cell to the local bit-line. Leakage from non-selected access transistors connect to the same local bit-line negatively affects the write SNM. Adding more cells to the local bit-line only increases the leakage. This, along with the increasingly poor $I_{O N} / I_{O F F}$ ratio as $V_{D D}$ decreases, also explains why there is a bigger change in write SNM among the different block configurations as $V_{D D}$ is lowered.

The differential traditional $8 \mathrm{~T}$ and standard $6 \mathrm{~T}$ cells have a much better write static noise margin at higher voltages, but the relative disadvantage diminishes as $V_{D D}$ drops. The differential write operation pulls down one node in the storage cell 


\section{Write SNM}

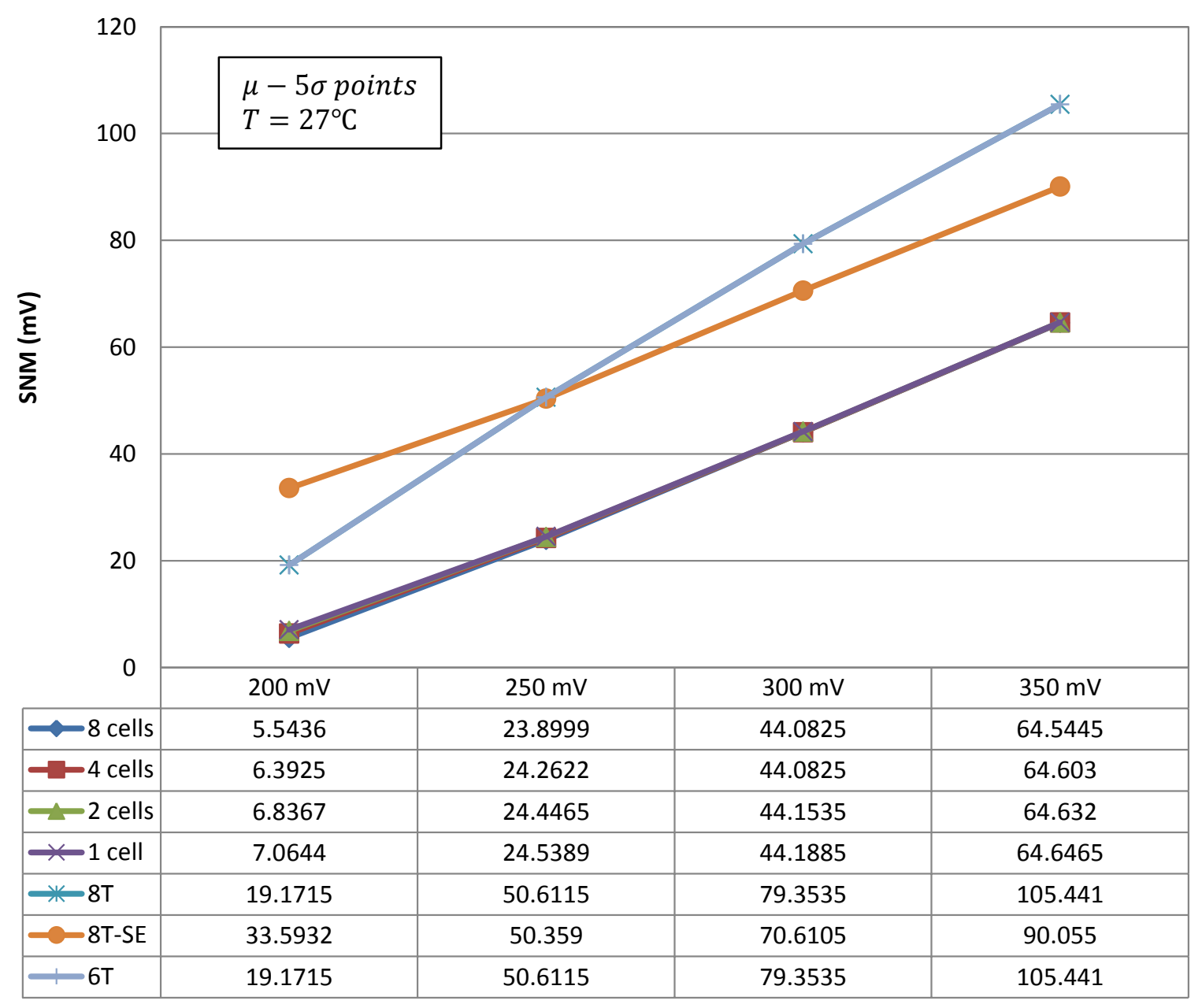

Figure 5.3: Simulation results for Write SNM vs. $V_{D D}$ for the different block configurations proposed, the traditional 8T cell, the traditional 8T cell with single-ended write operation, and the standard $6 \mathrm{~T}$ cell. The plotted points are $\mu-5 \sigma$. The points in the table are in $\mathrm{mV}$.

while pulling up the other. This allows for a higher write SNM compared to a singleended write operation. Unfortunately, as the voltage drops, and the $I_{O N} / I_{O F F}$ ratio decreases, the week pull-up is a disadvantage; to pull up the storage cell, the access transistor is enabled, which allows the leakage from other cells on the same bit-line to affect the node. However, at operational voltages for the proposed cell, the differential scheme always provides a better write SNM than the proposed architecture.

Peculiarly, the single-ended write operation for the traditional 8T cell (8T-SE in 
Figure 5.3) provides a better write static noise margin than the proposed design. This is intriguing because the write mechanisms are nearly identical. The connected bitline is brought low and one access transistor is enabled, pulling one side of the storage node down. The key difference is the use of a local bit-line and minimum-size writedecoupling transistor. Since all transistors inside the cell block are of minimum size, there is a slight contention between the load (or pull-up) transistor in the storage cell and the write decoupling transistor. Due to the use of NMOS access transistors and NMOS transistors being stronger than similarly sized PMOS transistors, the write decoupling transistor always wins, but the write operation is slightly weakened. In the traditional $8 \mathrm{~T}$ cell, the high capacitance global bit-lines connected to the cells require a strong pull-down transistor to discharge the bit-lines and keep the bit-line voltage relatively constant throughout the write operation. Thus, the write operation is stronger than in the proposed architecture.

The density function for the write operation in the proposed design is also dissimilar to the density functions of the $8 \mathrm{~T}$ and $6 \mathrm{~T}$ cells. Figure 5.4 shows a left-skewed normal distribution for the 8-cell configuration (again, only 1 configuration is included since they all have similar distributions). However, Figure 5.5 shows right-skewed normal distributions for the single-ended write operation of the traditional 8T SRAM cell and the differential write operation of the traditional $8 \mathrm{~T} /$ standard $6 \mathrm{~T}$ cell. This means that in extreme process variations the write operation actually improves in the $8 \mathrm{~T}$ and $6 \mathrm{~T}$ cells, unlike the hold and read cases. In the proposed design, the write operation gets worse in the presence of extreme process variations.

Hence, the worst case write noise margin of the $8 \mathrm{~T}$ and $6 \mathrm{~T}$ cells is close to the mean, and the noise margin actually improves in most cases when subjected to process variations. This is a very desirable property, which is not present in the proposed architecture. Conversely to the $8 \mathrm{~T}$ and $6 \mathrm{~T}$ cases, the best case write noise margin is close to the mean, and the noise margin worsens when subjected to process variations. This contrast is the result of connecting low capacitance local bit-lines to the storage cell instead of high-capacitance global bit-lines. Thus, while the local bit-lines help improve the read operation, it negatively impacts the write operation.

Figure 5.4 show that as $V_{D D}$ increases, the distribution becomes less skewed. The stronger on current, helps negate the negative impact of the local bit-lines, but not enough to have a right-skewed normal distribution like in the $8 \mathrm{~T}$ and $6 \mathrm{~T}$ cells. 


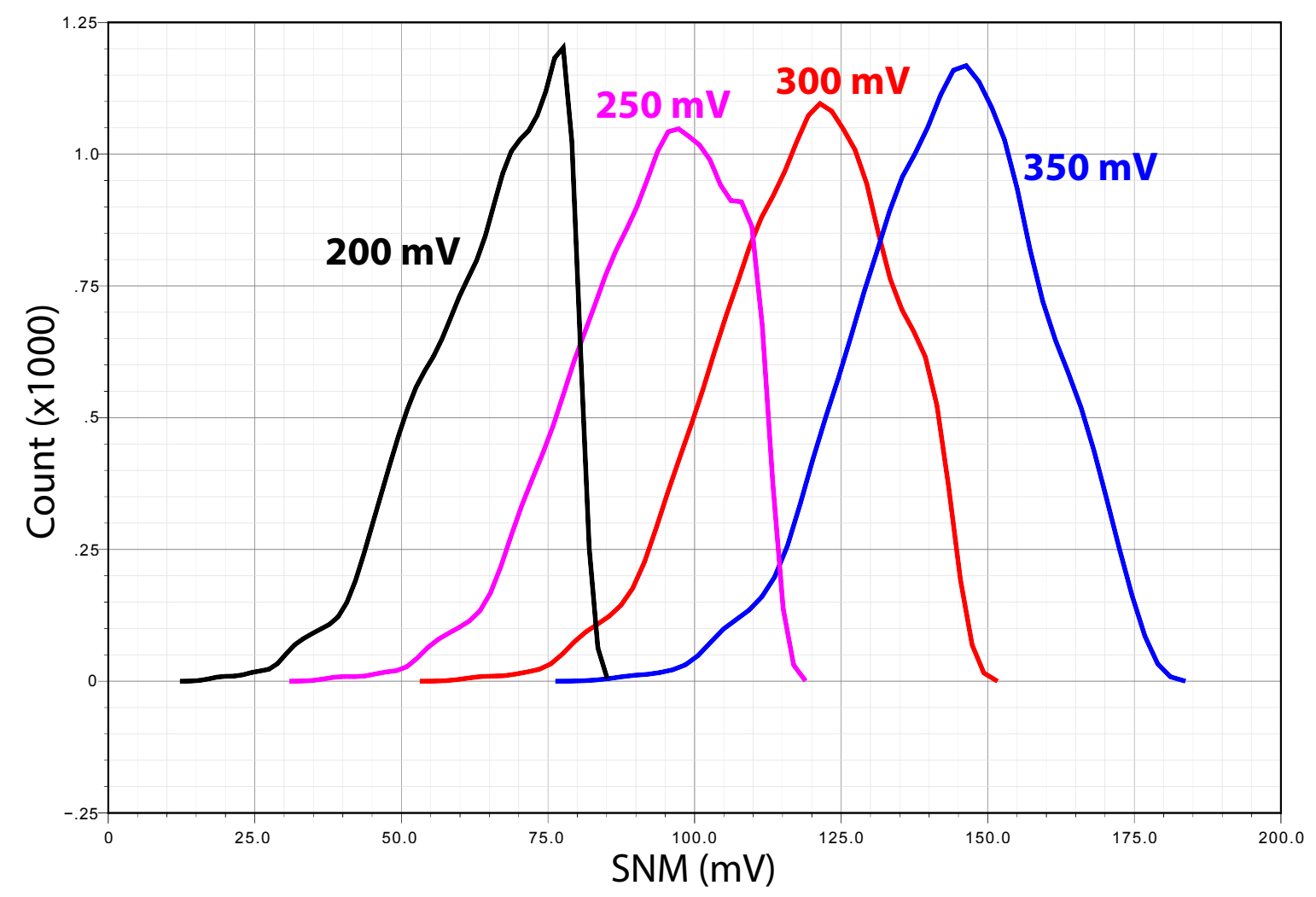

Figure 5.4: Estimated probably density function of the hold operation for the 8-cell block configuration at various supply voltages. The temperature is $27^{\circ} \mathrm{C}$. Only the 8-cell configuration is included because all configurations have very similar distributions. Refer to Figure A.4, Figure A.5, Figure A.6, and Figure A.7 for the histograms of the 8-cell configuration with supply voltages of $200 \mathrm{mV}, 250$ $\mathrm{mV}, 300 \mathrm{mV}$, and $350 \mathrm{mV}$, respectively.

\subsubsection{Read Operation}

The read operation is greatly affected by the number of bits in the cell block. Figure 5.6 displays that there is a large variation in the read static noise margin between each block configuration. At a $V_{D D}$ of $250 \mathrm{mV}$, the configuration with 8-cells has a read SNM of $11.878 \mathrm{mV}$, while the 1-cell configuration has a read SNM of $22.226 \mathrm{mV}$. Thus, as the number of bits in the cell block configuration increases, the read SNM decreases.

This diminishing SNM is the result of subjugating the storage node to the local bit-line. With the access transistor enabled, there is only one transistor separating 


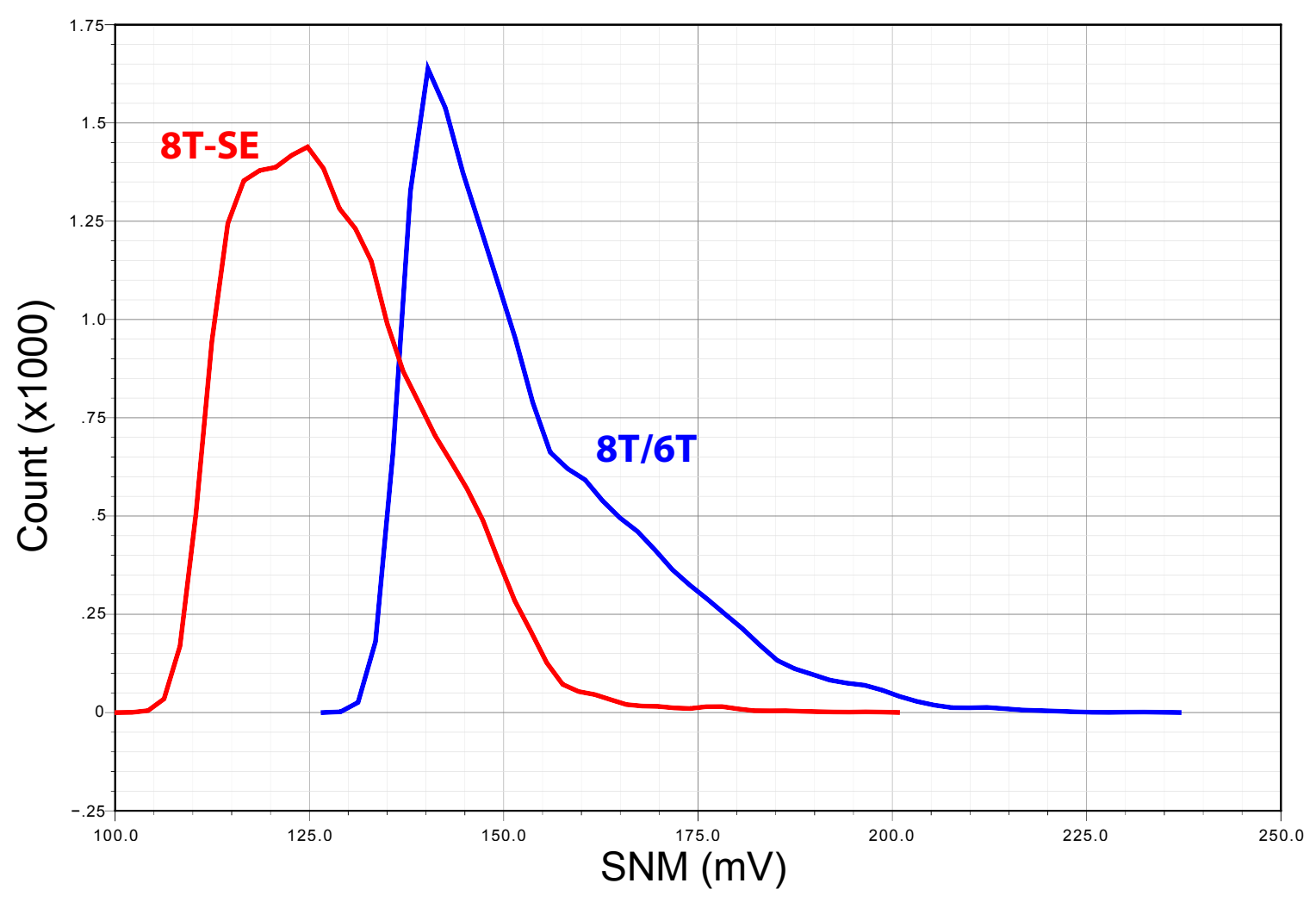

Figure 5.5: Estimated probably density function of the write operation for the $8 \mathrm{~T} / 6 \mathrm{~T}$ SRAM cell (they are the same), and $8 \mathrm{~T}$ single-ended write scheme. $V_{D D}$ is $300 \mathrm{mV}$ and the temperature is $27^{\circ} \mathrm{C}$. Only the the density function when the $V_{D D}$ is $300 \mathrm{mV}$ included because all supply voltages have very similar distributions. Refer to Figure A.8, and Figure A.9 for the histograms of the single-ended $8 \mathrm{~T}$, and $8 \mathrm{~T} / 6 \mathrm{~T}$ SRAM cells, respectively.

the storage node from the other storage nodes connected on the same local bit-line. Thus, leakage through the non-selected access transistors increases considerably. As more cells are added to the local bit-line, the total leakage into the local bit-line is further exacerbated.

This leakage is why the standard 6T SRAM cell has abysmal read static noise margins, as shown in Figure 5.6. In most 6T SRAM architectures, there are very large number of bits connected to the same bit-line. While this gives a high bit density, it greatly reduces the read SNM, causing it to fail at sub-threshold supply voltages. The proposed architecture doesn't have this issue since it limits the number of cells connected on the same bit-line. Furthermore, the proposed architecture only enables one access transistor during reads, which limits the disturbance from other 


\section{Read SNM}

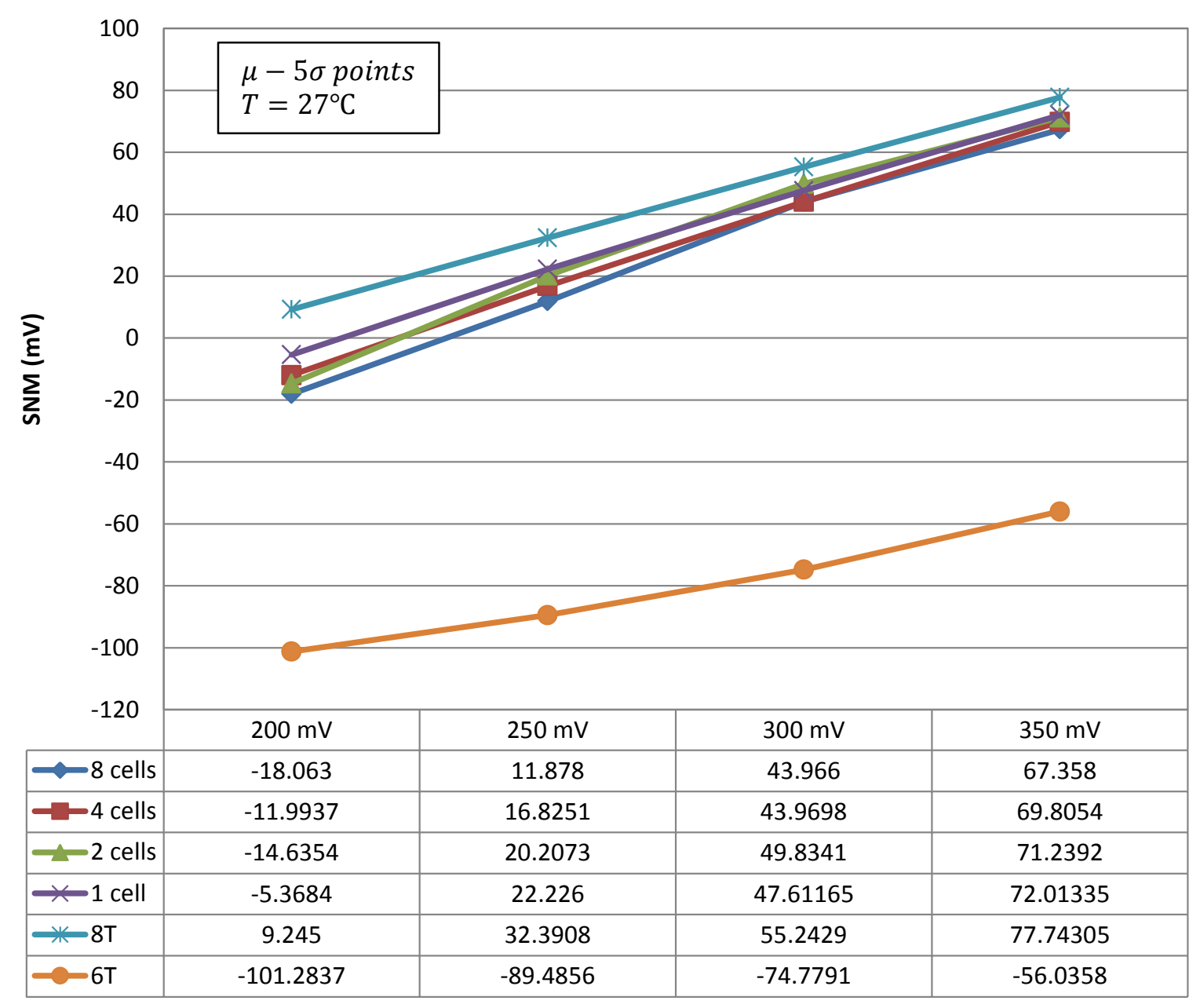

Figure 5.6: Simulation results for Read SNM vs. $V_{D D}$ for the different block configurations proposed, the traditional $8 \mathrm{~T}$ cell, and the standard $6 \mathrm{~T}$ cell. The plotted points are $\mu-5 \sigma$. The points in the table are in $\mathrm{mV}$.

storage cells to a single node.

The conventional 8T SRAM cell has a slightly higher read SNM compared to the proposed architecture due to the storage cells being isolated from the bit-lines during reads. The read SNM is exactly the same as the hold SNM because the access transistors are off during the read operation. This also results in the 8T read operation being less affected by a reduction in $V_{D D}$. Figure 5.6 shows that the proposed architecture has a larger reduction in read SNM than the $8 \mathrm{~T}$ cell as $V_{D D}$ is lowered. There is a further decrease in read SNM as the number of bits in the cell 
block configuration is increased.

Analyzing the density functions in Figure 5.7 shows that the traditional $8 \mathrm{~T}$ cell and the proposed design both have left-skewed normal distributions and are very similar. The standard $6 \mathrm{~T}$ cell has a very slightly right-skewed normal distribution. The read SNM for the $6 \mathrm{~T}$ cell is very poor and can vary wildly due to the lack of stability.

Based on the simulation results, it is obvious that the read static noise margin is the limiting factor in the block configuration. Hence, there is a comprise between integration density and read SNM (and by extension minimum operating voltage). Depending on the yield and noise tolerance required, when using the 65nm LP TSMC process, $250 \mathrm{mV}$ would be the minimum operating voltage for the 8-cell configuration and $230 \mathrm{mV}$ for the 1-cell configuration.

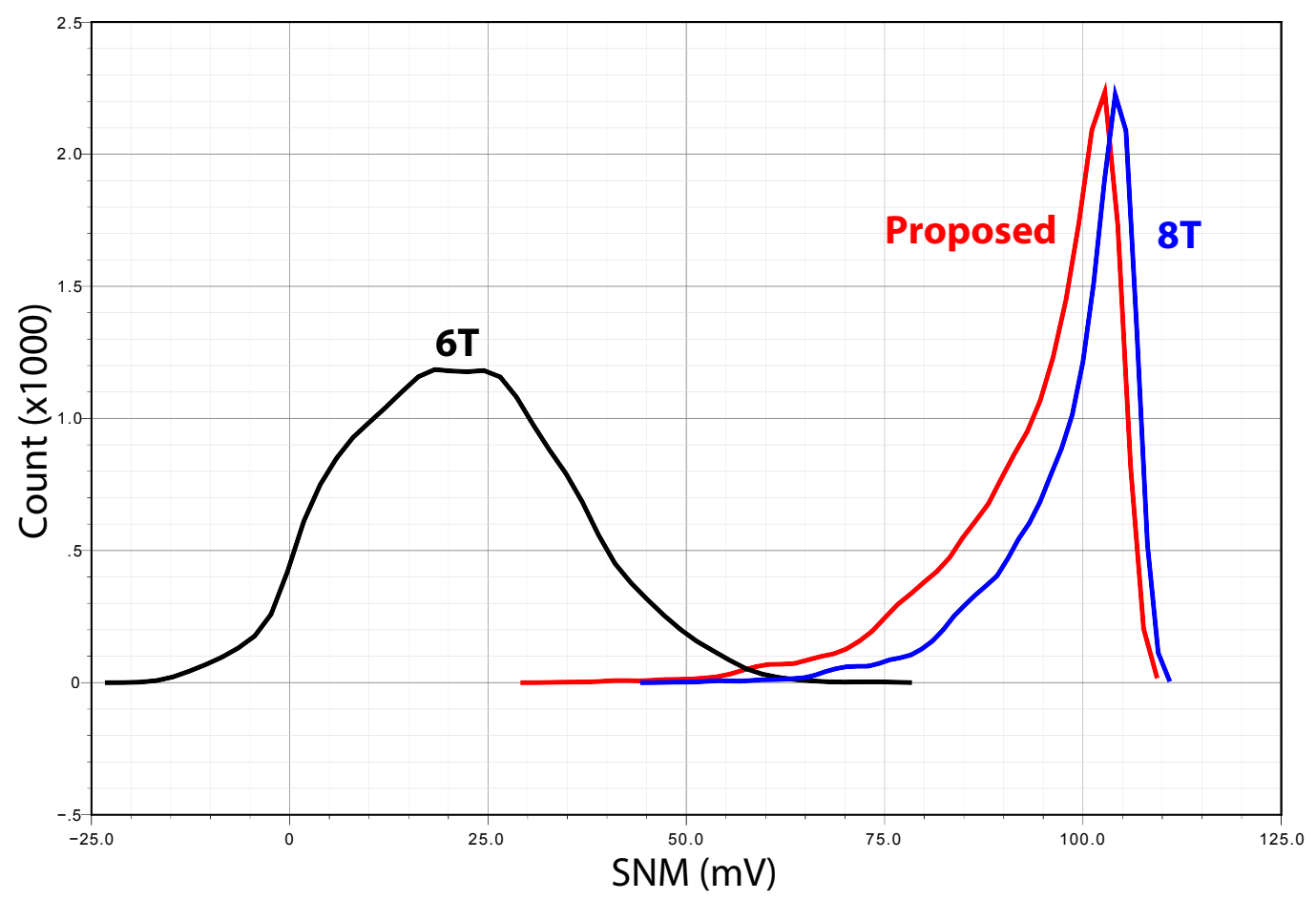

Figure 5.7: Estimated probably density function of the read operation for the 8-cell block configuration, $8 \mathrm{~T}$, and $6 \mathrm{~T}$ SRAM cells. $V_{D D}$ is $300 \mathrm{mV}$ and the temperature is $27^{\circ} \mathrm{C}$. Only the 8-cell configuration is included because all configurations have very similar distributions. This is similarly true for all sub-threshold operating voltages. Refer to Figure A.10, Figure A.11, and Figure A.12 for the histograms of the 8-cell block configuration, 8T SRAM cell, and 6T SRAM cell, respectively. 


\subsection{Temperature Variations}

The equations in 4.1 show that the sub-threshold current depends on several temperature-dependent parameters and, thus, varies with temperature. The temperature dependence on the threshold voltage, $V_{T}$, can also be expressed by

$$
V_{T H}=V_{T H 0}-\kappa T,
$$

where $V_{T H 0}$ is the threshold voltage at $0 \mathrm{~K}, T$ is the absolute temperature, and $\kappa$ is the temperature coefficient of $V_{T H}$. From this equation, it is clear that the threshold voltage decreases as the temperature increases [50]. Furthermore, the mobility, $\mu$, of the MOSFET carriers can be expressed by

$$
\mu(T)=\mu\left(T_{0}\right)\left(T / T_{0}\right)^{-m},
$$

where $\mu\left(T_{0}\right)$ is the carrier mobility at room temperature $T_{0}$, and $m$ is the mobility temperature exponent. This equation shows that the mobility of the carriers decreases as the temperature increases [50].

By utilizing equations 4.1,5.1, and 5.2, the temperature coefficient of the the subthreshold current of a MOSFET with a fixed gate-to-source voltage and $V_{D S}>0.1 \mathrm{~V}$ (making the current relatively independent of $V_{D S}$ ) can be found. The temperature coefficient is as follows:

$$
\begin{aligned}
T . C \cdot \text { Sub } & =\frac{1}{I_{\text {sub }}} \frac{d I_{\text {sub }}}{d T} \\
& =\frac{1}{\mu} \frac{d \mu}{d T}+\frac{1}{V_{T}^{2}} \frac{d V_{T}^{2}}{d T}+\frac{1}{e^{\frac{\left(V_{G S}-V_{T H}\right)}{n V_{T}}} \frac{d}{d T} e^{\frac{V_{G S}-V_{T H}}{n V_{T}}}} \\
& =\frac{2-m}{T}-\frac{1}{n V_{T}} \frac{\left(V_{G S}-V_{T H 0}\right)}{T}
\end{aligned}
$$

Thus, the sub-threshold current has a positive temperature coefficient and increases with temperature [50].

Given this temperature dependence, there can be a lot of variance in the SNM at differing temperatures. As such, it is important to evaluate the stability of the proposed SRAM design at multiple temperatures. The following test simulations show that the TSMC $65 \mathrm{~nm}$ LP process has increased variations at low temperatures. Thus, 
to more accurately show temperature trends, box plots are used to show temperature alterations. The central mark in the box plots is the median, and the edges of the box are the 25th and 75th percentiles. The whiskers are defined as larger than $q 3+1.5(q 3-q 1)$ or smaller than $q 1-1.5(q 3-q 1)$, where $\mathrm{q} 1$ and $\mathrm{q} 3$ are the 25 th and 75 th percentiles. This corresponds to approximately $\pm 2.7 \sigma$ (or $99.3 \%$ ) coverage when the data is normally distributed [51], [49].

\subsubsection{Hold SNM}

As shown in Figure 5.8, Figure 5.9, Figure 5.10, and Figure 5.11, the temperature trends are the same for all block configurations. There isn't much change in the hold SNM as we increase the number of bits. The hold SNM remains the same in all block configurations at the same temperatures. As the temperature increases, there is a notable decrease in SNM due to the increase in leakage current. Unexpectedly, below $0{ }^{\circ} \mathrm{C}$ the $\mathrm{SNM}$ decreases as the temperature drops. The variability of the SNM decreases as the temperature increases.

Examining Figure 5.12 and Figure 5.13 show that the increase in variability in the hold SNM experienced in the proposed SRAM cell as the temperature drops similarly occurs in the traditional $8 \mathrm{~T}$ and standard $6 \mathrm{~T}$ SRAM cells. It can be seen that the standard deviation in the proposed SRAM cell is roughly equal to the 8T SRAM cell at temperatures greater than or equal to $0^{\circ} \mathrm{C}$, but at below $0^{\circ} \mathrm{C}$ the standard deviation is larger. The difference in standard deviation further increases as the temperature drops. This is a result of there being additional transistors on the local bit-line that affect leakage. The affect of temperature on the hold SNM of the SRAM cells is summarized in Figure 5.14. 


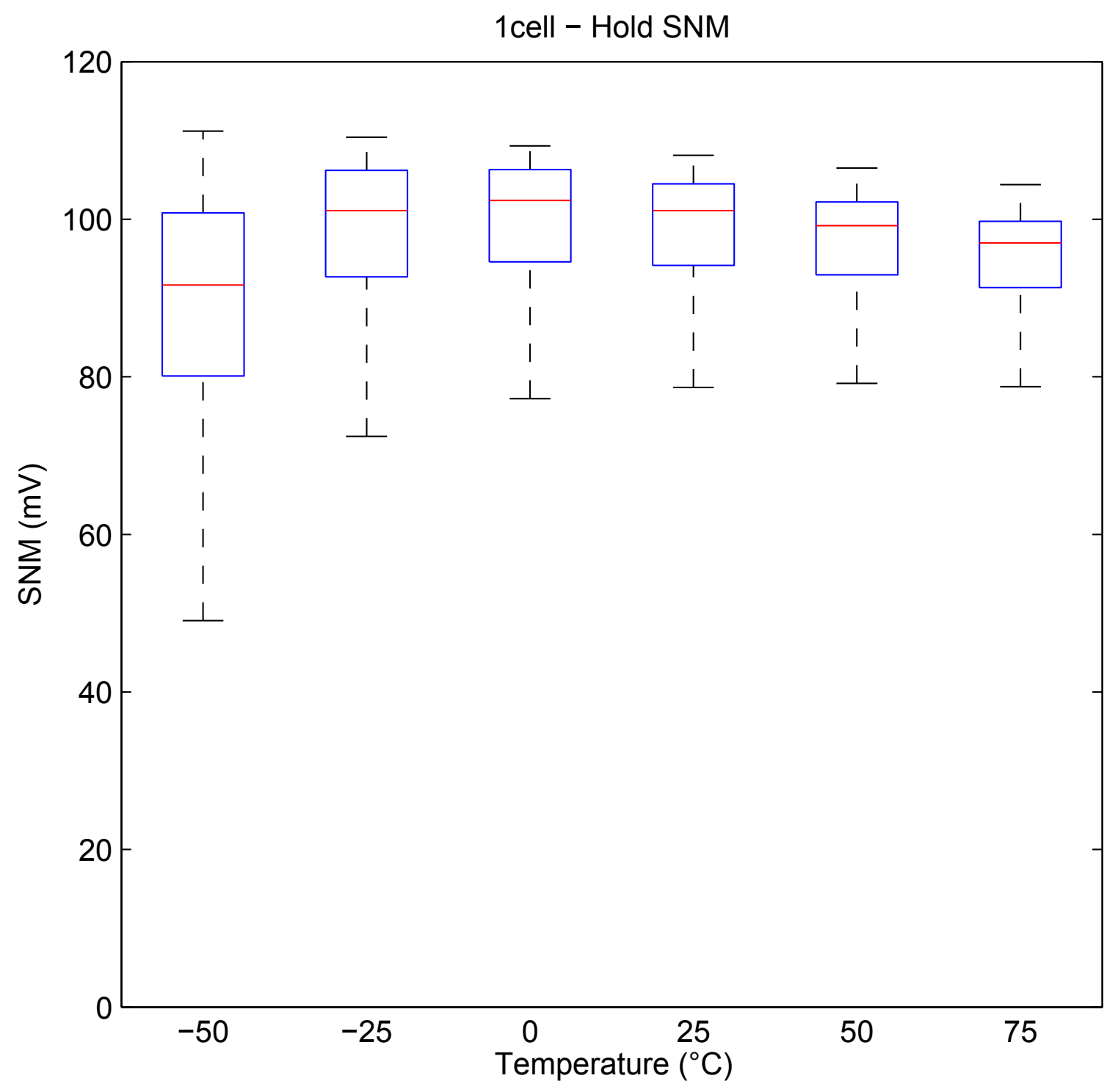

Figure 5.8: Hold SNM vs. Temperature $\left({ }^{\circ} \mathrm{C}\right)$ for the 1-cell block. 


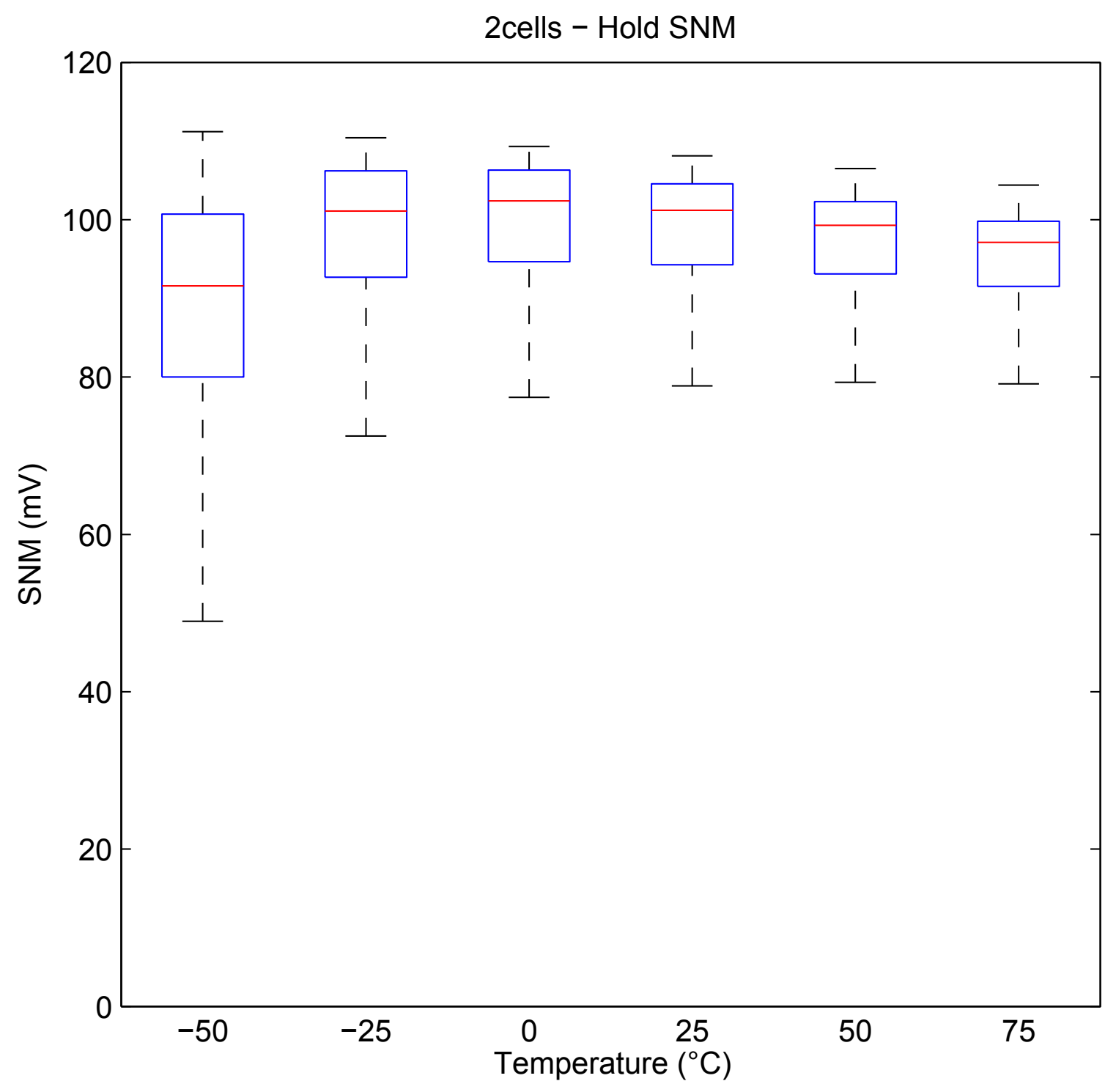

Figure 5.9: Hold SNM vs. Temperature $\left({ }^{\circ} \mathrm{C}\right)$ for the 2-cell block. 


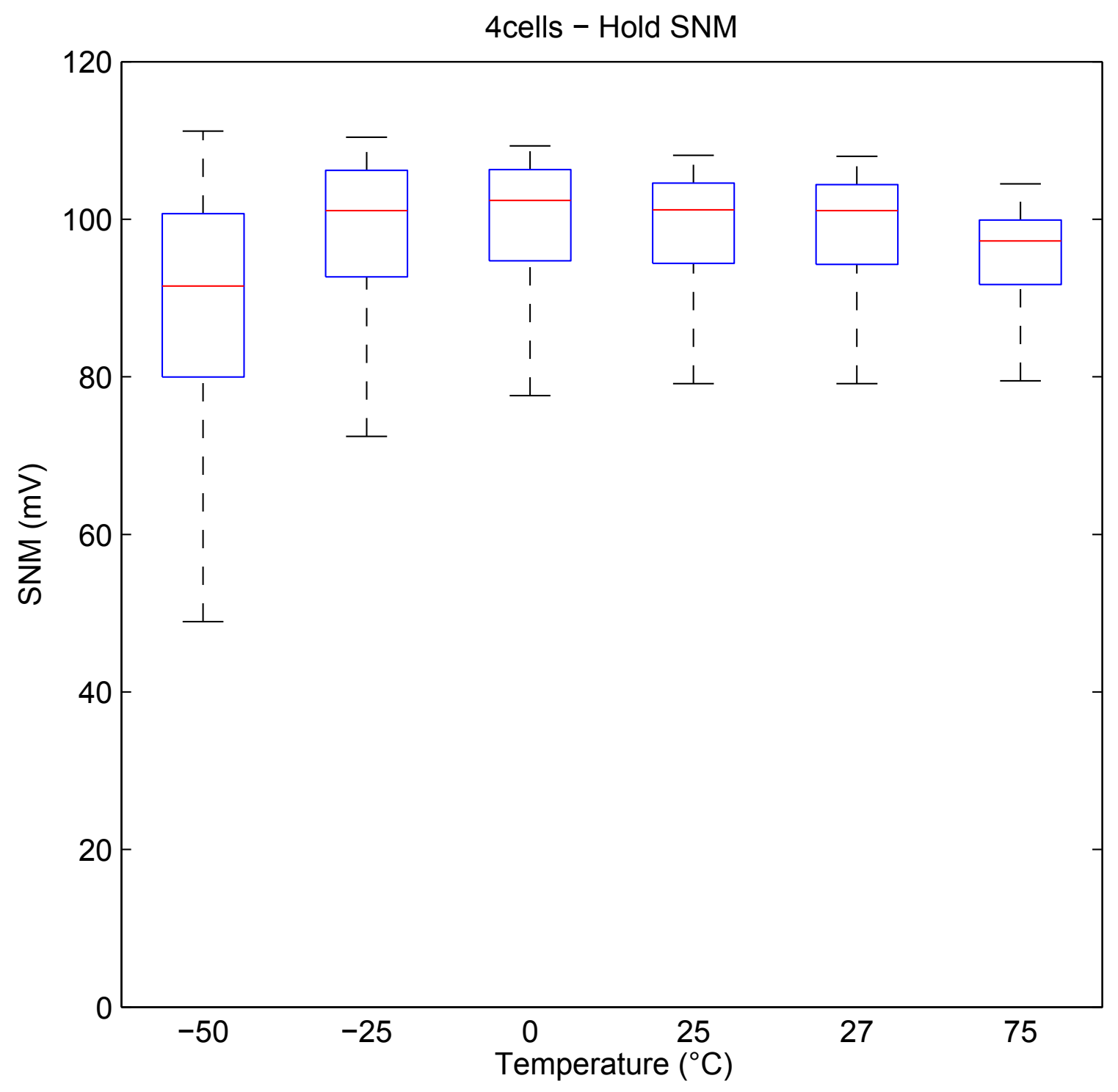

Figure 5.10: Hold SNM vs. Temperature $\left({ }^{\circ} \mathrm{C}\right)$ for the 4-cell block. 


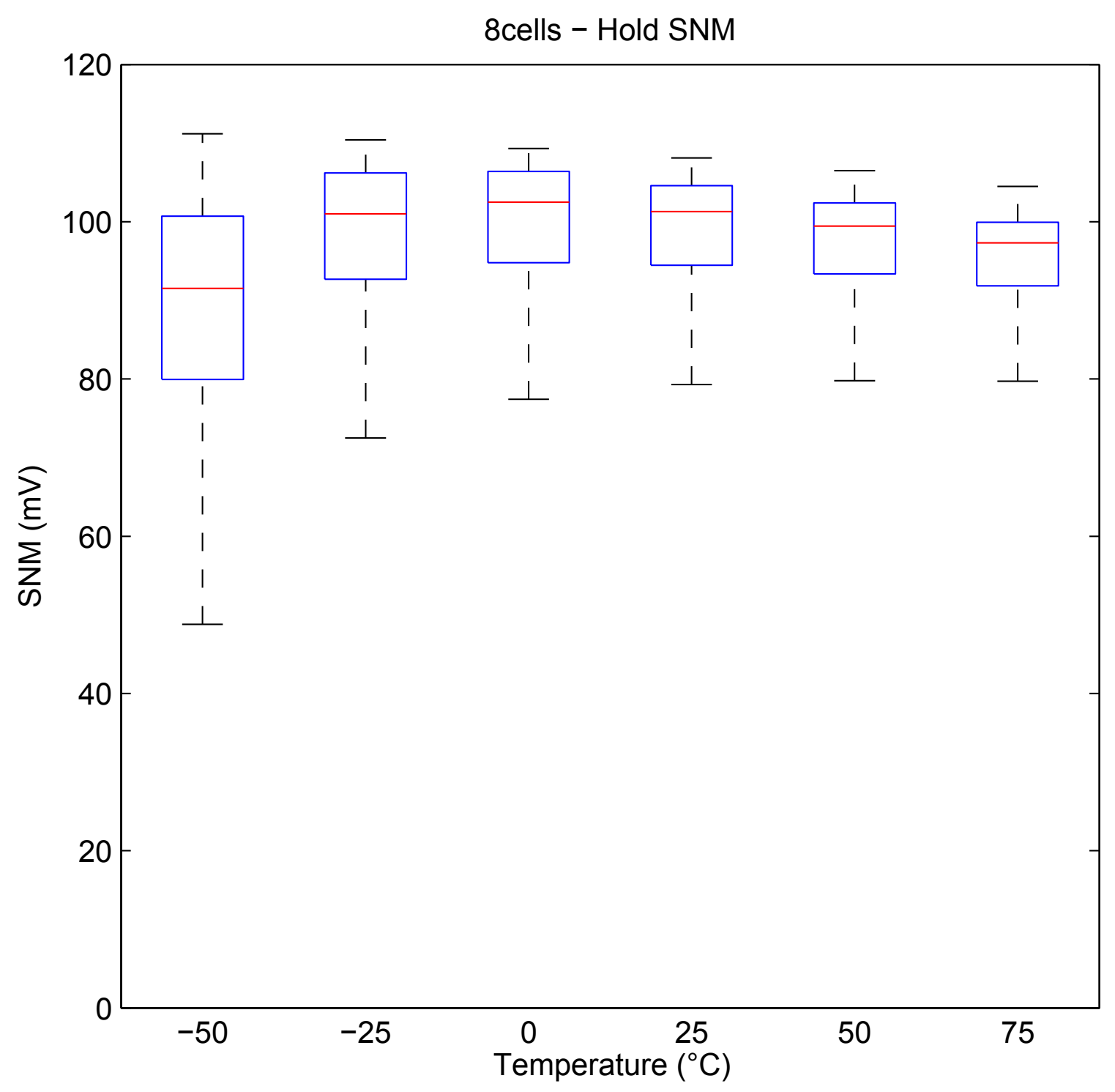

Figure 5.11: Hold SNM vs. Temperature $\left({ }^{\circ} \mathrm{C}\right)$ for the 8-cell block. 


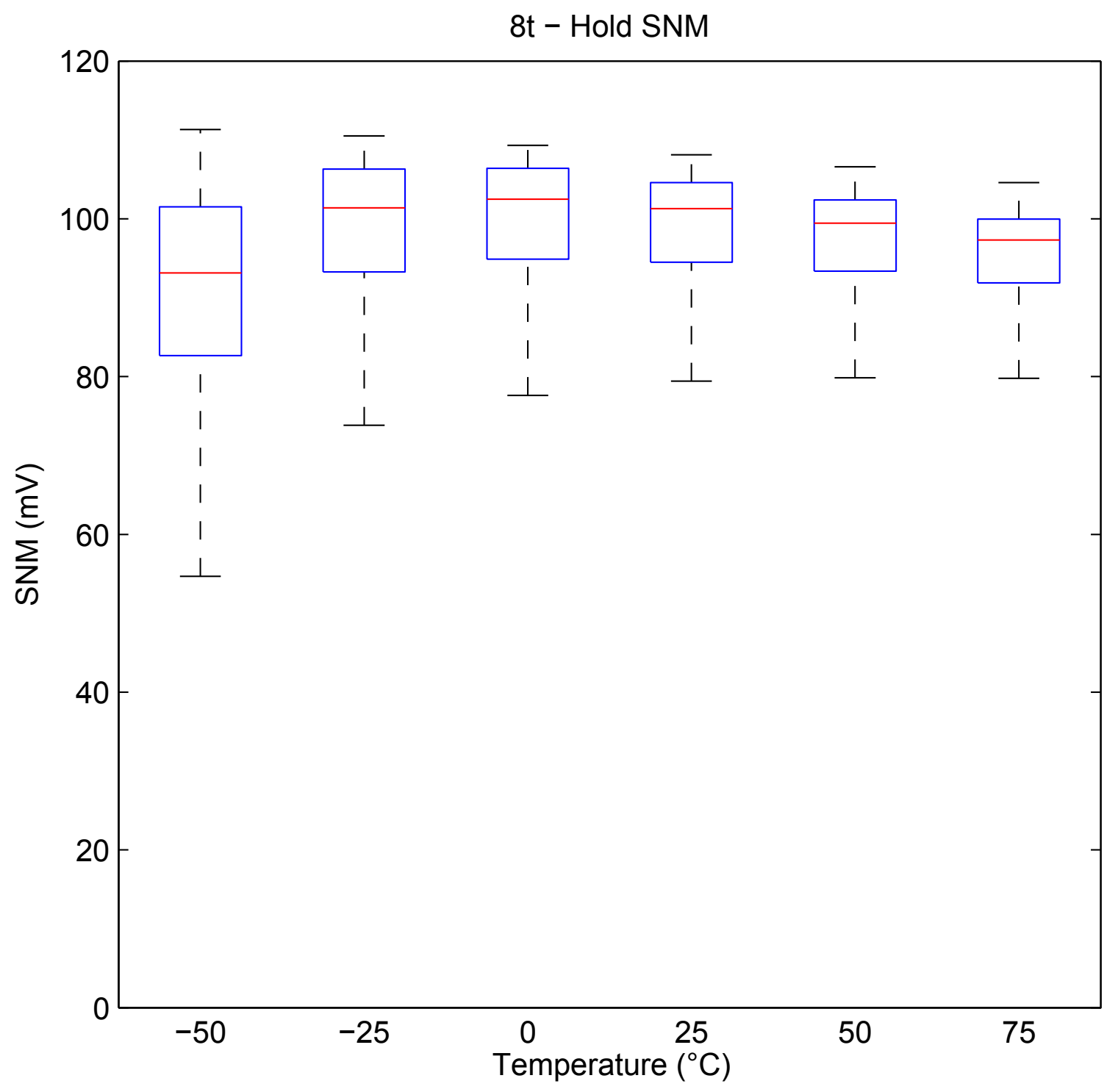

Figure 5.12: Hold SNM vs. Temperature $\left({ }^{\circ} \mathrm{C}\right)$ for the traditional $8 \mathrm{~T}$ SRAM cell. 


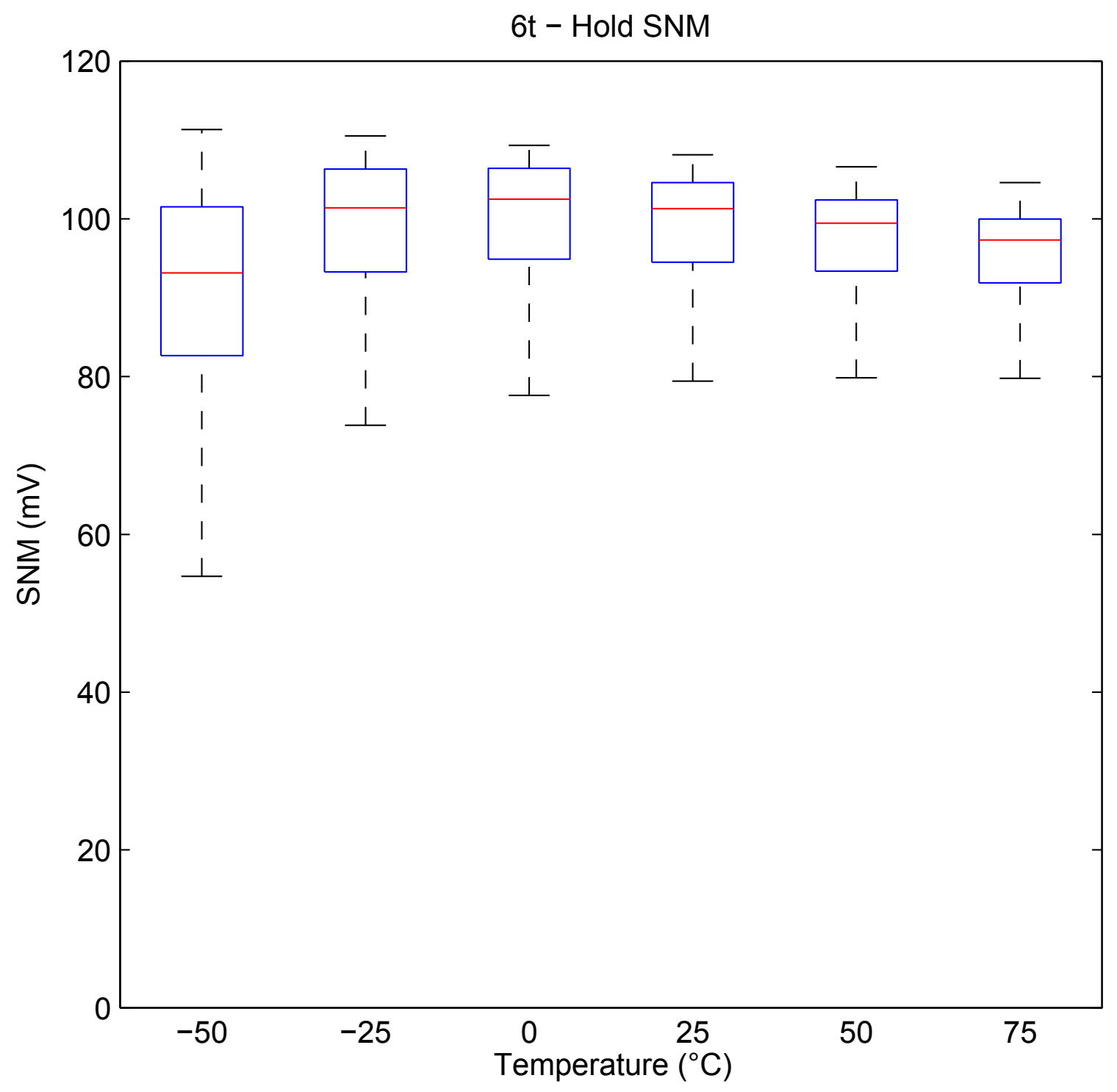

Figure 5.13: Hold SNM vs. Temperature $\left({ }^{\circ} \mathrm{C}\right)$ for the standard $6 \mathrm{~T}$ SRAM cell. 


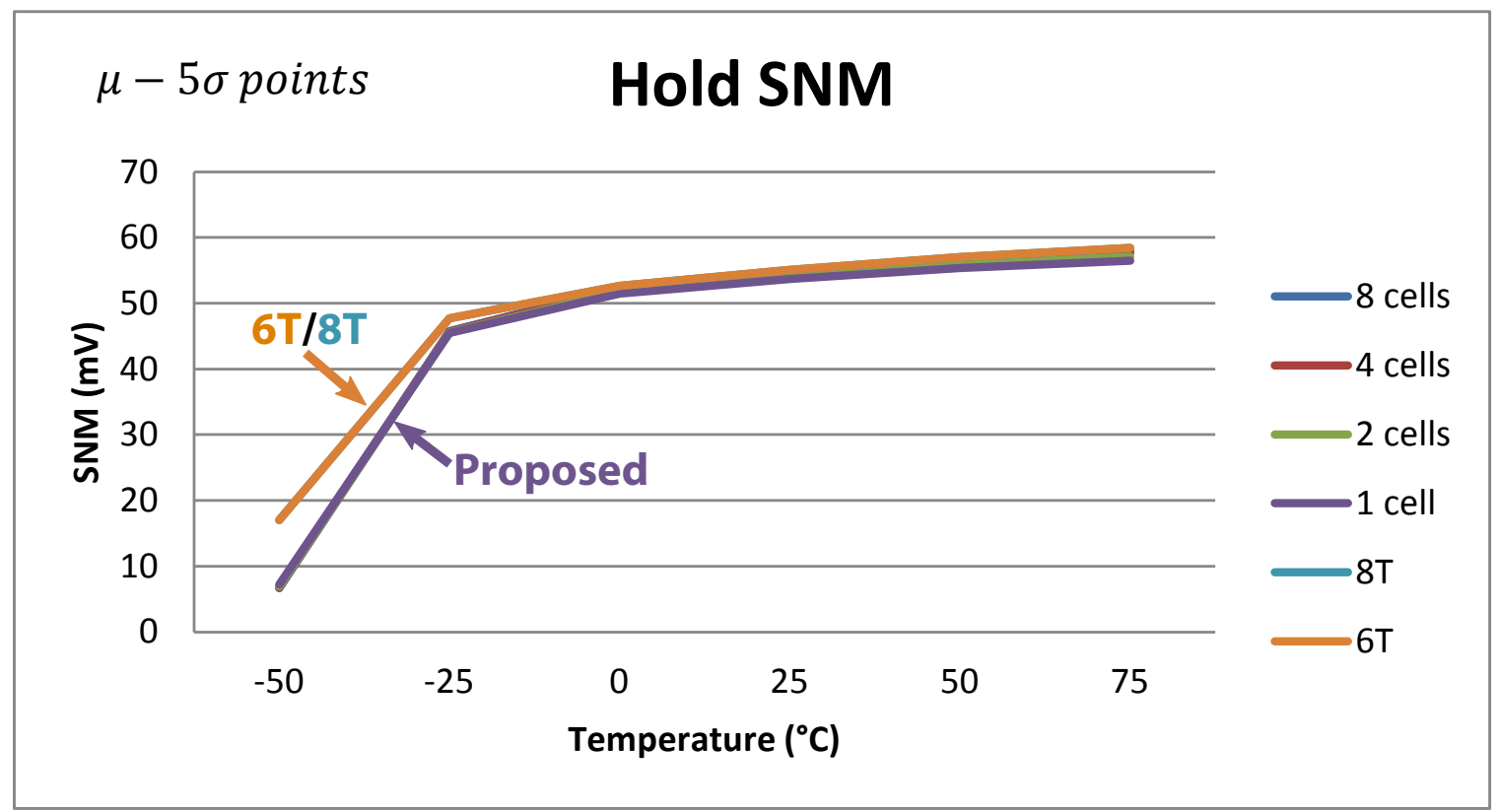

Figure 5.14: Simulation results for Hold SNM vs. Temperature $\left({ }^{\circ} \mathrm{C}\right)$ for the different block configurations proposed, the traditional $8 \mathrm{~T}$ cell, and the standard $6 \mathrm{~T}$ cell. $V_{D D}$ is $300 \mathrm{mV}$. The plotted points are $\mu-5 \sigma$. 


\subsubsection{Read SNM}

The temperature has a greater affect on the read SNM than on the hold SNM. Figure 5.15, Figure 5.16, Figure 5.17, and Figure 5.18 show that the read SNM of the proposed SRAM cell decreases as the temperature drifts above or below $0^{\circ} \mathrm{C}$. Once again, the variability decreases as the temperature increases. The standard deviation drastically increases and the average SNM decreases exponentially as the temperature drops below $0^{\circ} \mathrm{C}$.

The temperature has a greater affect on the read SNM as the number of bits increase inside the block. When the temperature drops below $0^{\circ} \mathrm{C}$, there is a significant decrease in the average SNM and a much greater variability as the number of bits in the block increases. However, when increasing the temperature above $0^{\circ} \mathrm{C}$, there is not a significant change in the average SNM and standard deviation as the number of bits increases. There is a minor drop in the average SNM and a small increase in the standard deviations when additional bits are added to the block. These changes can be attributed to the storage cell being exposed to the local bit-line on reads. Additional cells in the cell block increases the number of transistors that are affected by temperature variations.

Comparing Figure 5.18 and Figure 5.19 shows that temperature has a greater impact on the read SNM of the proposed design than on the traditional 8T SRAM cell. Compared to the 8T SRAM cell, there is increased variability at all temperatures. The increase in standard deviation is especially pronounced below $0^{\circ} \mathrm{C}$. There is a larger decrease in average SNM in the proposed design than in the 8T cell as the temperature deviates from $0^{\circ} \mathrm{C}$. This decrease is more prominent with each additional bit added to the block configuration. Again, this can be attributed to the storage cell being exposed to the local bit-line, whereas, in the 8T SRAM cell, the storage cell is isolated during reads.

Temperature has relatively little affect on the read SNM of the standard 6T SRAM cell at sub-threshold voltages, as shown in Figure 5.20. The change in the average SNM as the temperature changes is decently larger in the proposed SRAM architecture than in the 6T SRAM cell. The proposed SRAM architecture also has lesser 


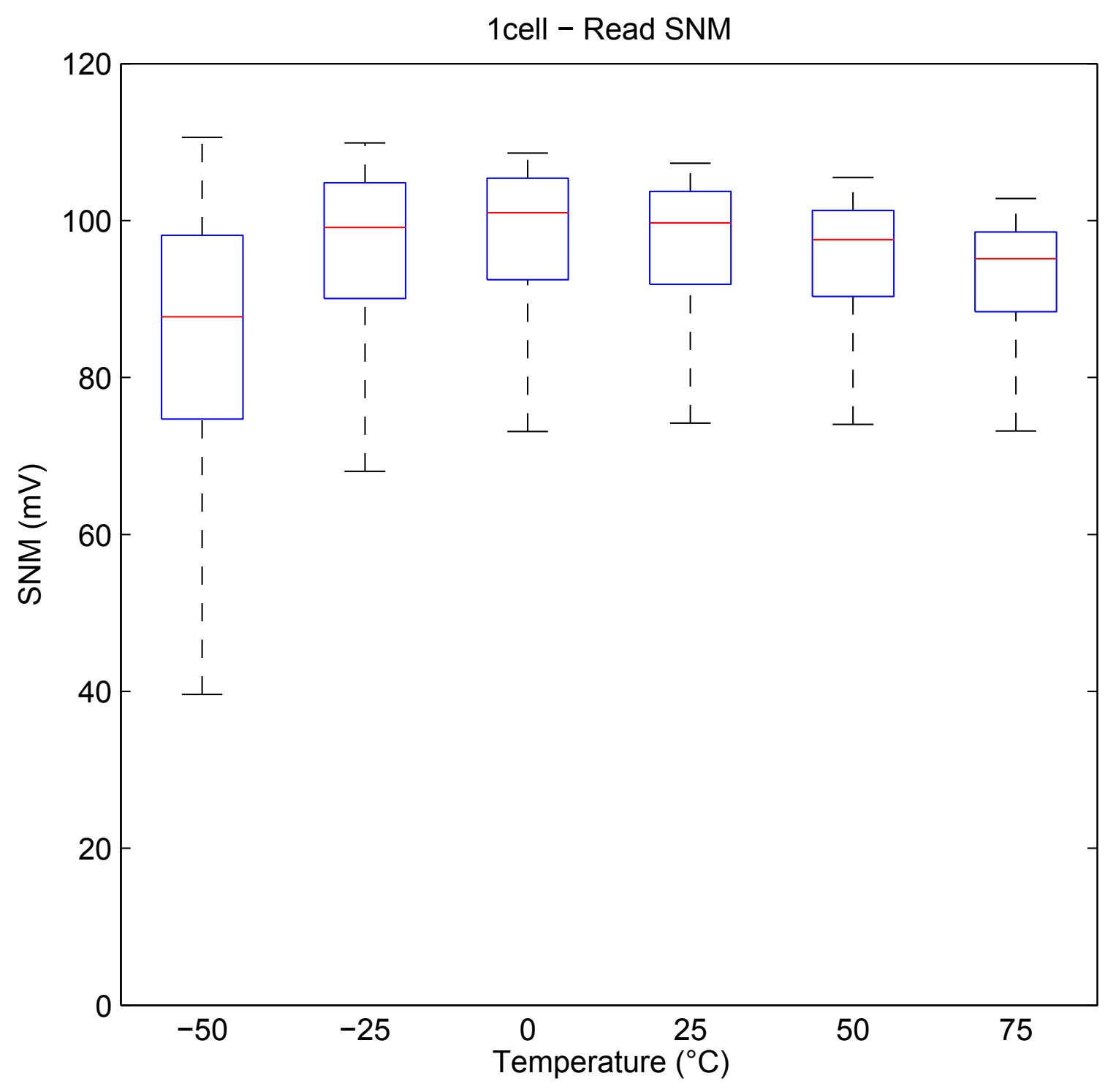

Figure 5.15: Read SNM vs. Temperature $\left({ }^{\circ} \mathrm{C}\right)$ for the 1-cell block. 


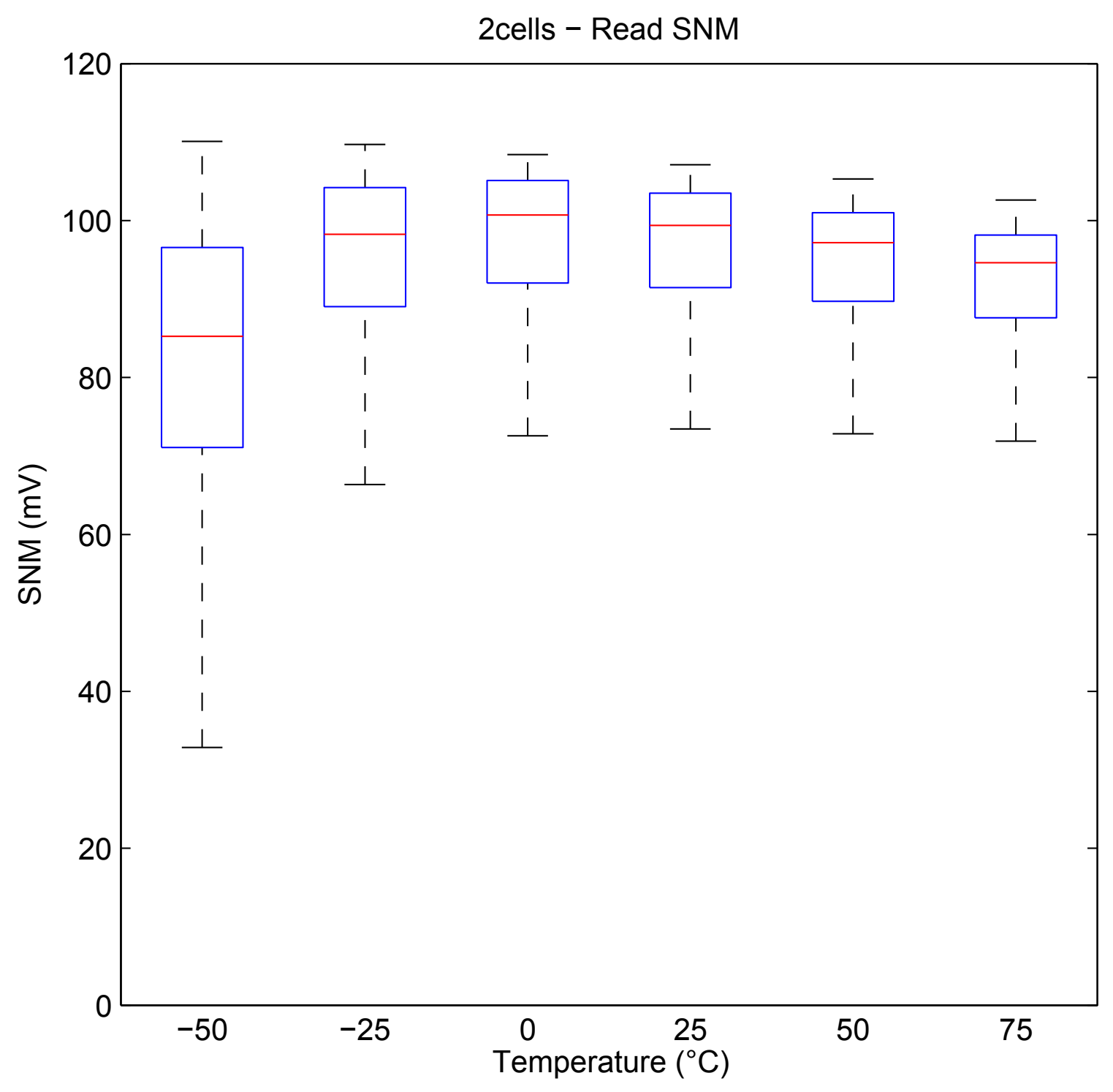

Figure 5.16: Read SNM vs. Temperature $\left({ }^{\circ} \mathrm{C}\right)$ for the 2-cell block. 


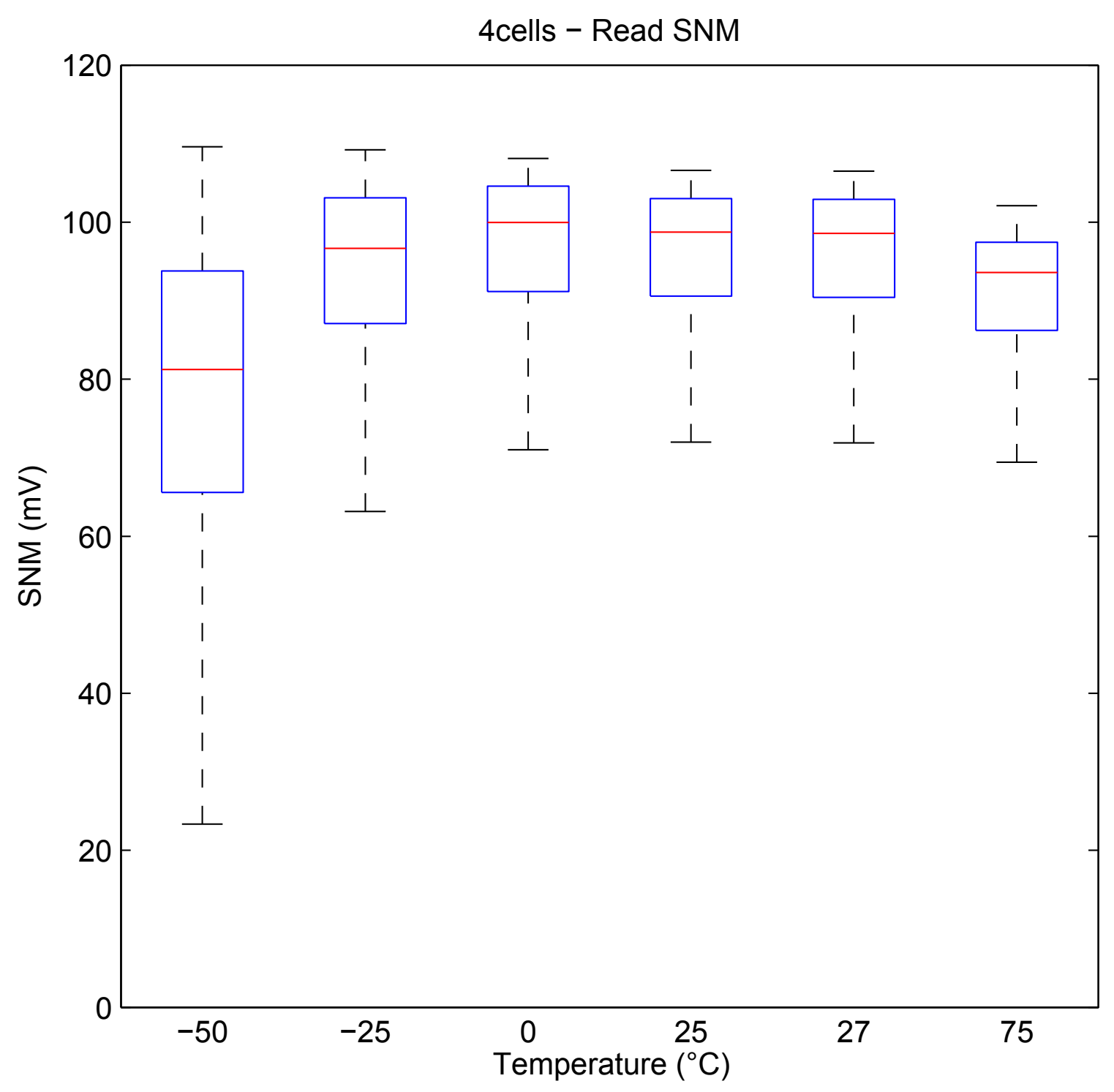

Figure 5.17: Read SNM vs. Temperature $\left({ }^{\circ} \mathrm{C}\right)$ for the 4-cell block. 


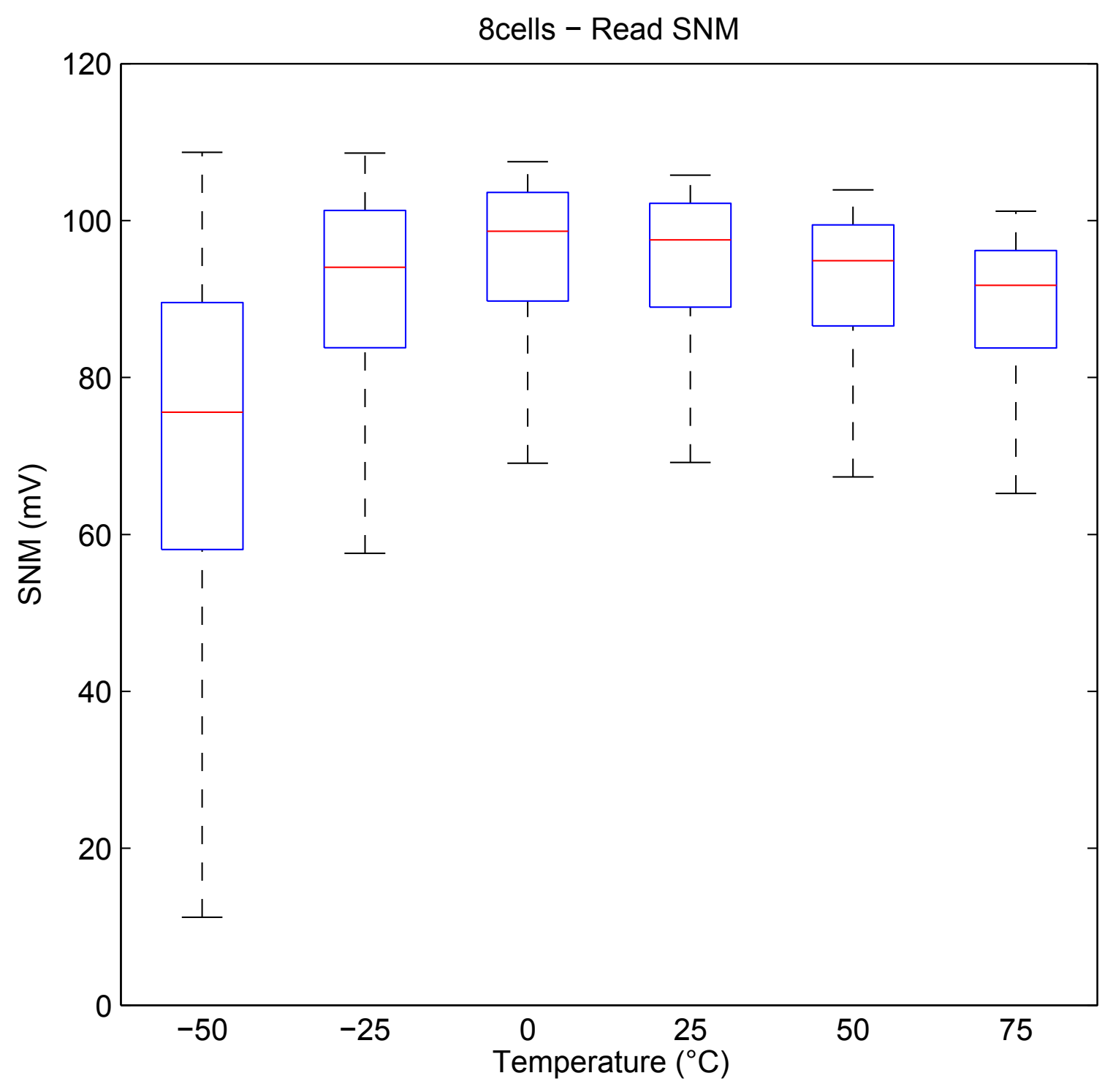

Figure 5.18: Read SNM vs. Temperature $\left({ }^{\circ} \mathrm{C}\right)$ for the 8-cell block. 


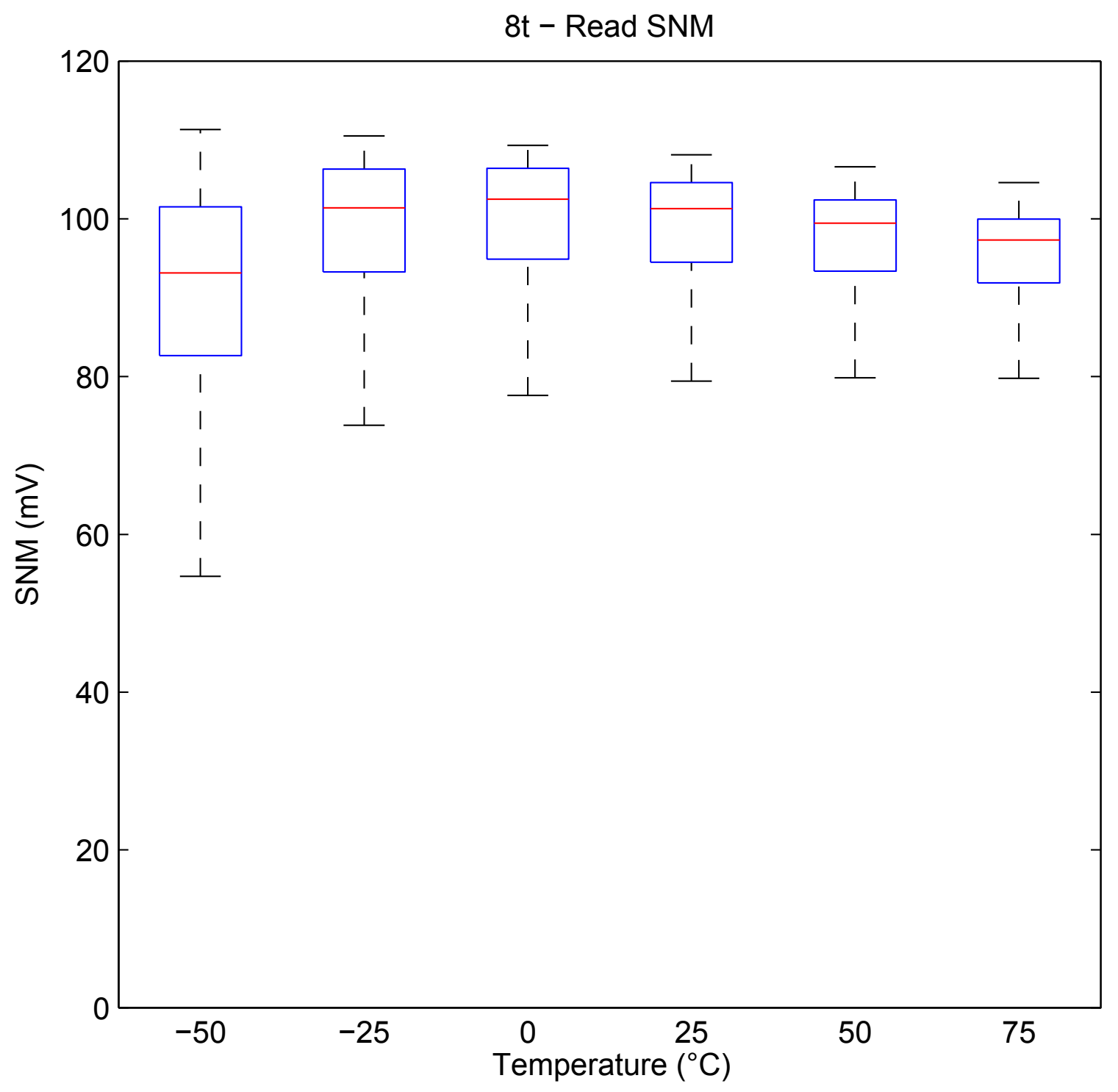

Figure 5.19: Read SNM vs. Temperature $\left({ }^{\circ} \mathrm{C}\right)$ for the traditional $8 \mathrm{~T}$ SRAM cell. 


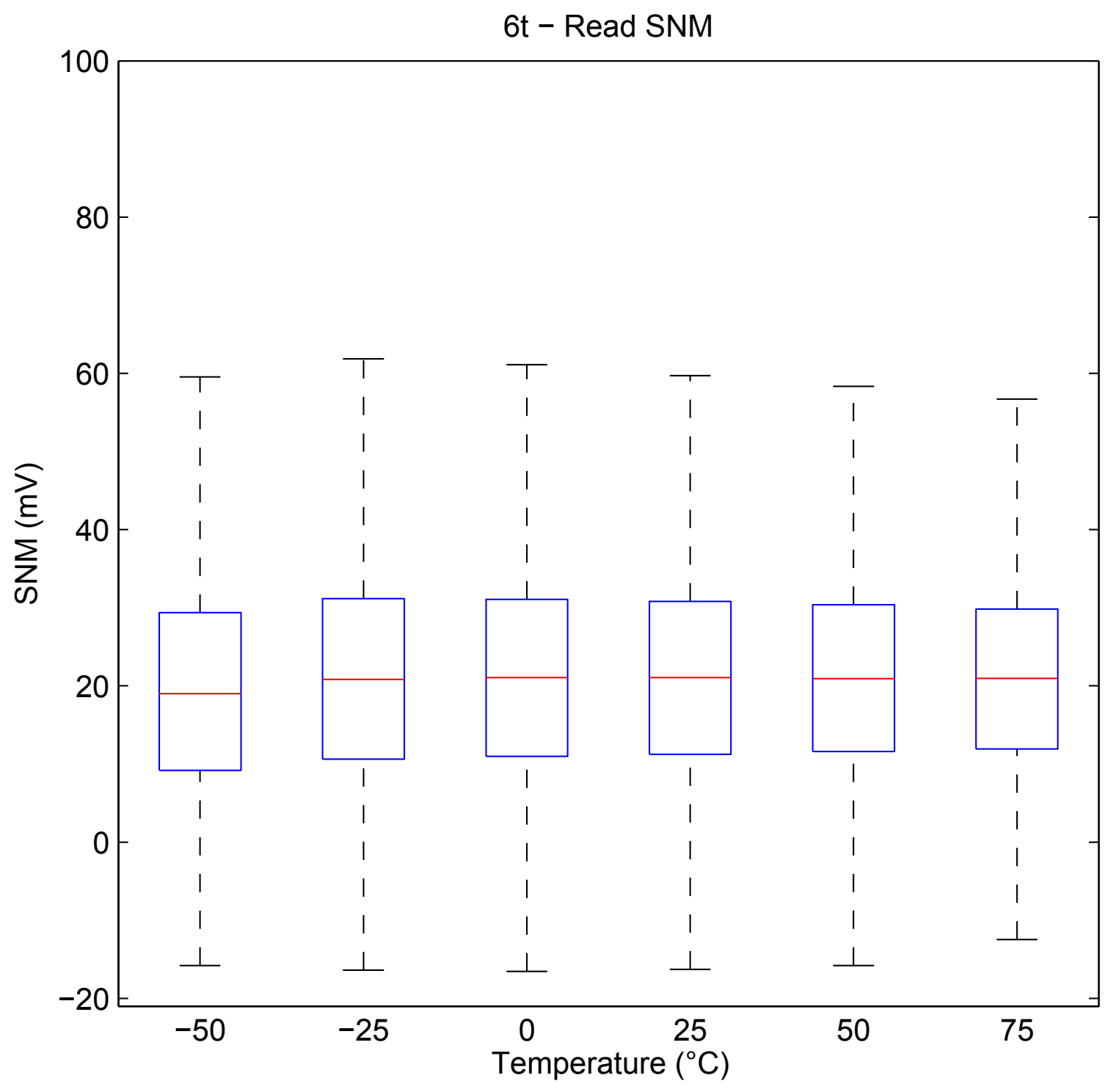

Figure 5.20: Read SNM vs. Temperature $\left({ }^{\circ} \mathrm{C}\right)$ for the standard $6 \mathrm{~T}$ SRAM cell. Note that the y-axis has been translated down $20 \mathrm{mV}$ to show the standard deviation without changing the scale. 
variability at all temperatures, except at $-50^{\circ} \mathrm{C}$, compared to the $6 \mathrm{~T}$ SRAM cell. The variability in the $6 \mathrm{~T}$ SRAM cell remains more or less the same at all temperatures, with a slight decrease as the temperature moves away from $0^{\circ} \mathrm{C}$. This is in stark contrast with the proposed design which has a noticeable decrease in average SNM as the temperature diverges from $0^{\circ} \mathrm{C}$, and a decrease in a standard deviation as the temperature increases. This shows how the 6T SRAM cell is less stable at sub-threshold voltages compared to the proposed design and the 8T SRAM cell. This difference is due to the use of the relatively short local bit-line. The long global bit-lines utilized in the 6T SRAM cell subjects the storage cell to all the PVT variations in all the cells connected to the bit-line.

Curiously, the 6T SRAM cell has an even spread above and below the median, whereas the proposed SRAM cell (and 8T SRAM cell) have an increased spread below the median. This means in the average case, the read SNM is close to the upper limit, and only in extreme process variations does the read SNM decrease. The affect of temperature on the read SNM of the SRAM cells is summarized in Figure 5.21.

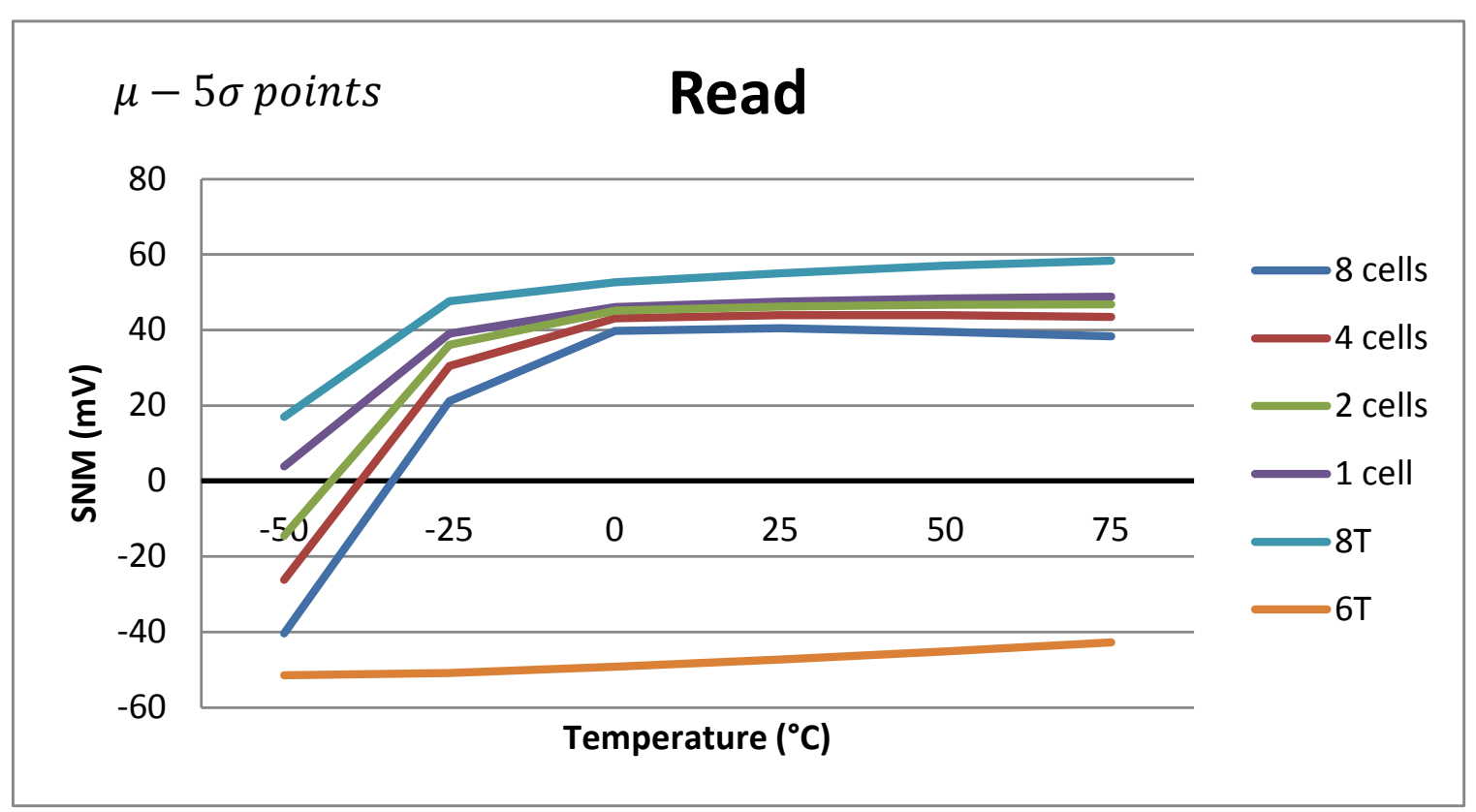

Figure 5.21: Simulation results for Read SNM vs. Temperature $\left({ }^{\circ} \mathrm{C}\right)$ for the different block configurations proposed, the traditional $8 \mathrm{~T}$ cell, and the standard $6 \mathrm{~T}$ cell. $V_{D D}$ is $300 \mathrm{mV}$. The plotted points are $\mu-5 \sigma$. 


\subsubsection{Write SNM}

The write operation is more resilient to temperature variations than the read and hold operations. Figure 5.22, Figure 5.23, Figure 5.24, and Figure 5.25 show that as the temperature changes there is relatively little change in the average write SNM of the proposed Design. Additionally, there is only a minor increase in variability as the temperature drops.

Since the write SNM is not dependent on the number of bits in each block, the temperature has an equivalent affect on all block configurations. As is consistent with the read and write static noise margins, there is a decrease in the average write SNM as the temperature deviates from $0^{\circ} \mathrm{C}$. However, the decrease is not as significant. Interestingly, the rate of decrease of the average SNM below $0^{\circ} \mathrm{C}$ is not as substantial as in the read and write cases. This is true for the increase of variability as well. This is evident by comparing Figure 5.11, Figure 5.18, and Figure 5.25.

Temperature variations have a smaller affect on the write operation of the proposed design than they do on the write operation in the traditional 8T and standard 6T SRAM cells. Figure 5.25 shows that the average SNM and variability of the proposed design slightly decreases as the temperature diverges from $0^{\circ} \mathrm{C}$. However, Figure 5.26 and Figure 5.28 show that the median SNM and standard deviation of the $8 \mathrm{~T}$ and $6 \mathrm{~T}$ cells have a greater reduction than the proposed design as the temperature decreases.

Comparing Figure 5.25 and Figure 5.27 shows that the write operation of the proposed SRAM cell is not as tolerant of temperature variations as the single-ended write operation in traditional $8 \mathrm{~T}$ SRAM cells. Compared to the single-ended write scheme, there is a larger decrease in average SNM and increased variability in the proposed design as the temperature drifts from $0^{\circ} \mathrm{C}$. In contrast to $8 \mathrm{~T}$ SRAM cells utilizing a single-ended write scheme, as the temperature drops below $0^{\circ} \mathrm{C}$ there is a decrease in the write SNM of the proposed SRAM cell. Thus, it can be stated that the write operation for the proposed cell follows the same trend as the $8 \mathrm{~T}$ read and hold operations instead of following the single-ended write operation. The single-ended 


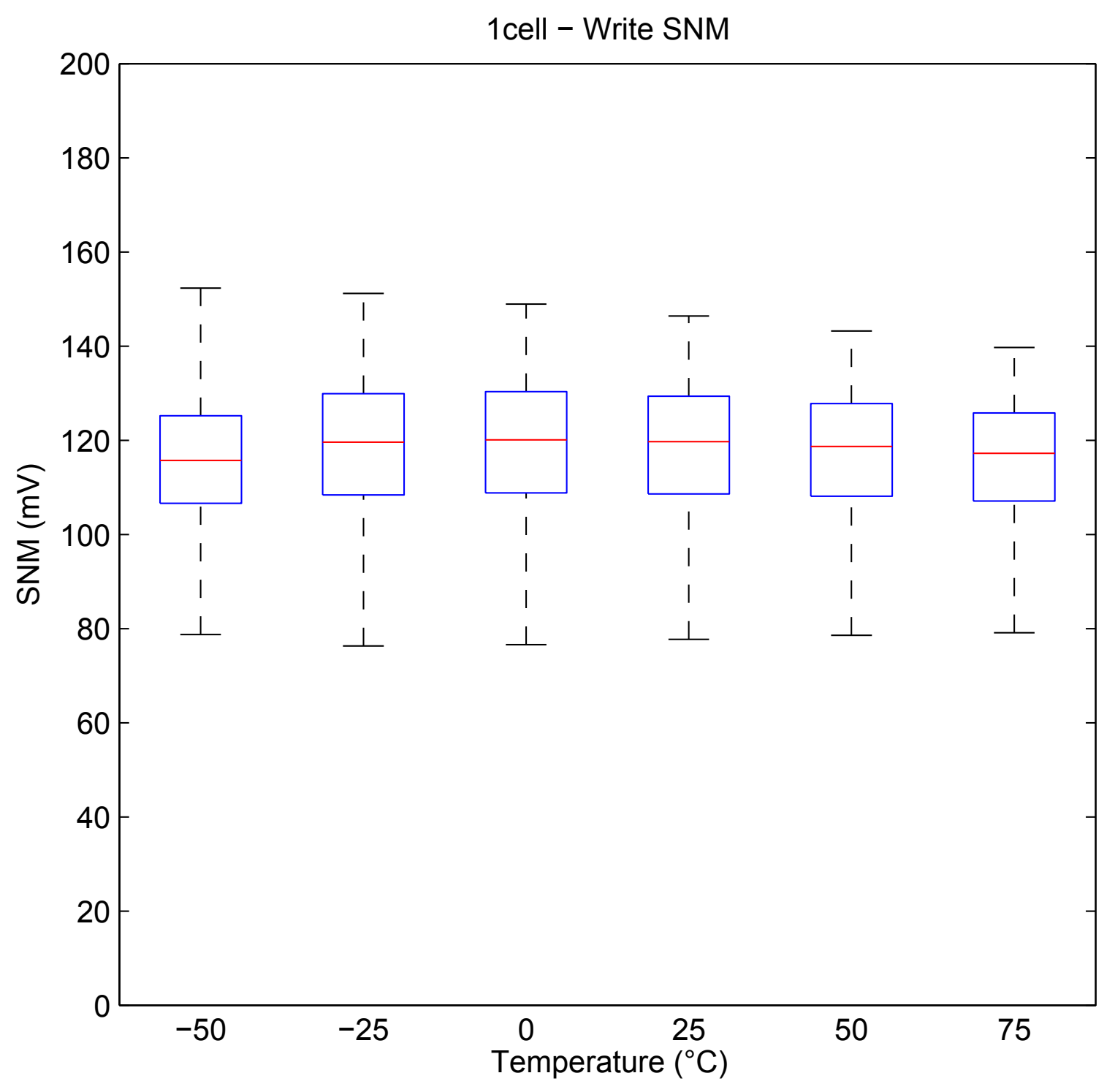

Figure 5.22: Write SNM vs. Temperature $\left({ }^{\circ} \mathrm{C}\right)$ for the 1-cell block. 


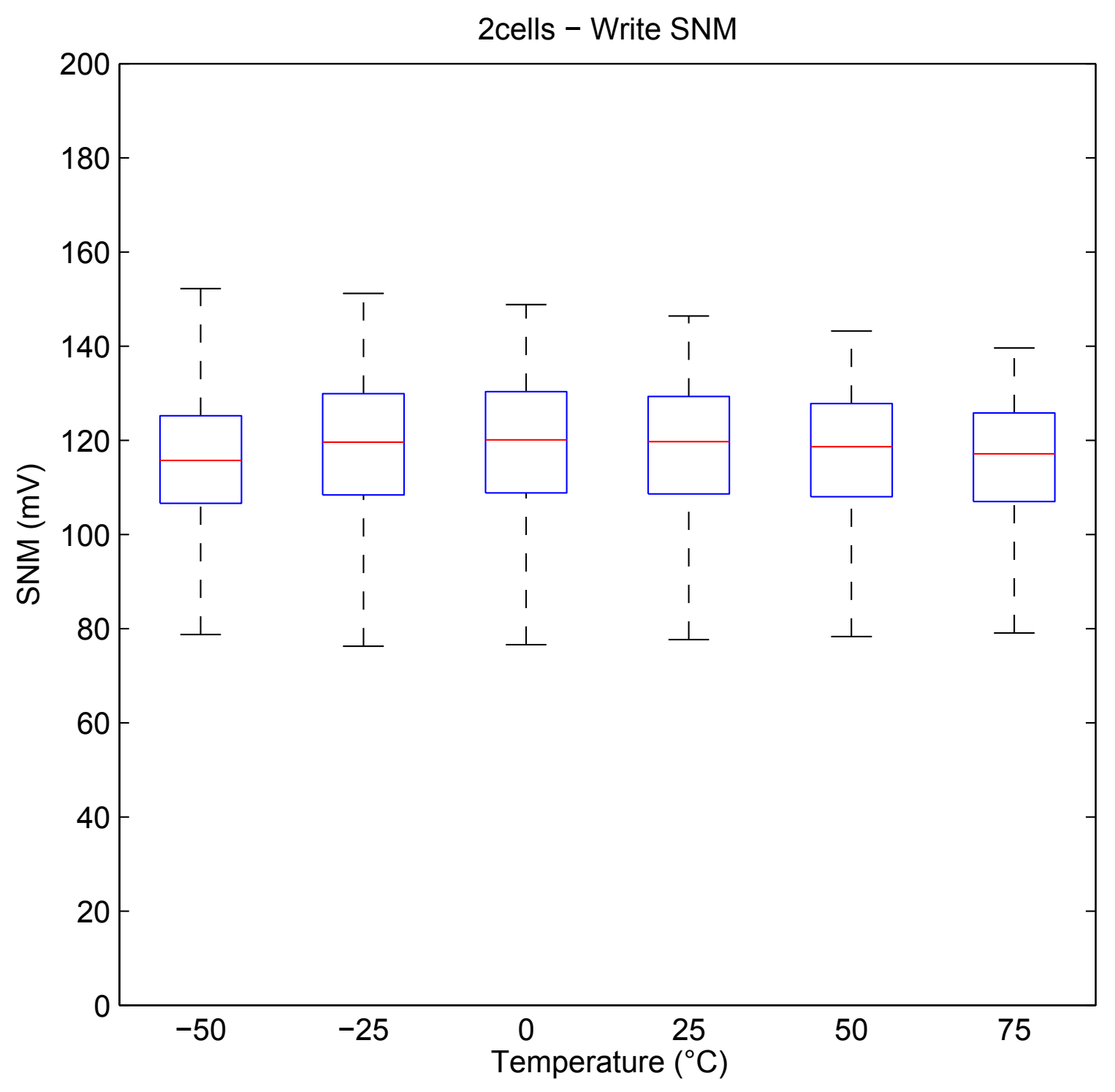

Figure 5.23: Write SNM vs. Temperature $\left({ }^{\circ} \mathrm{C}\right)$ for the 2-cell block. 


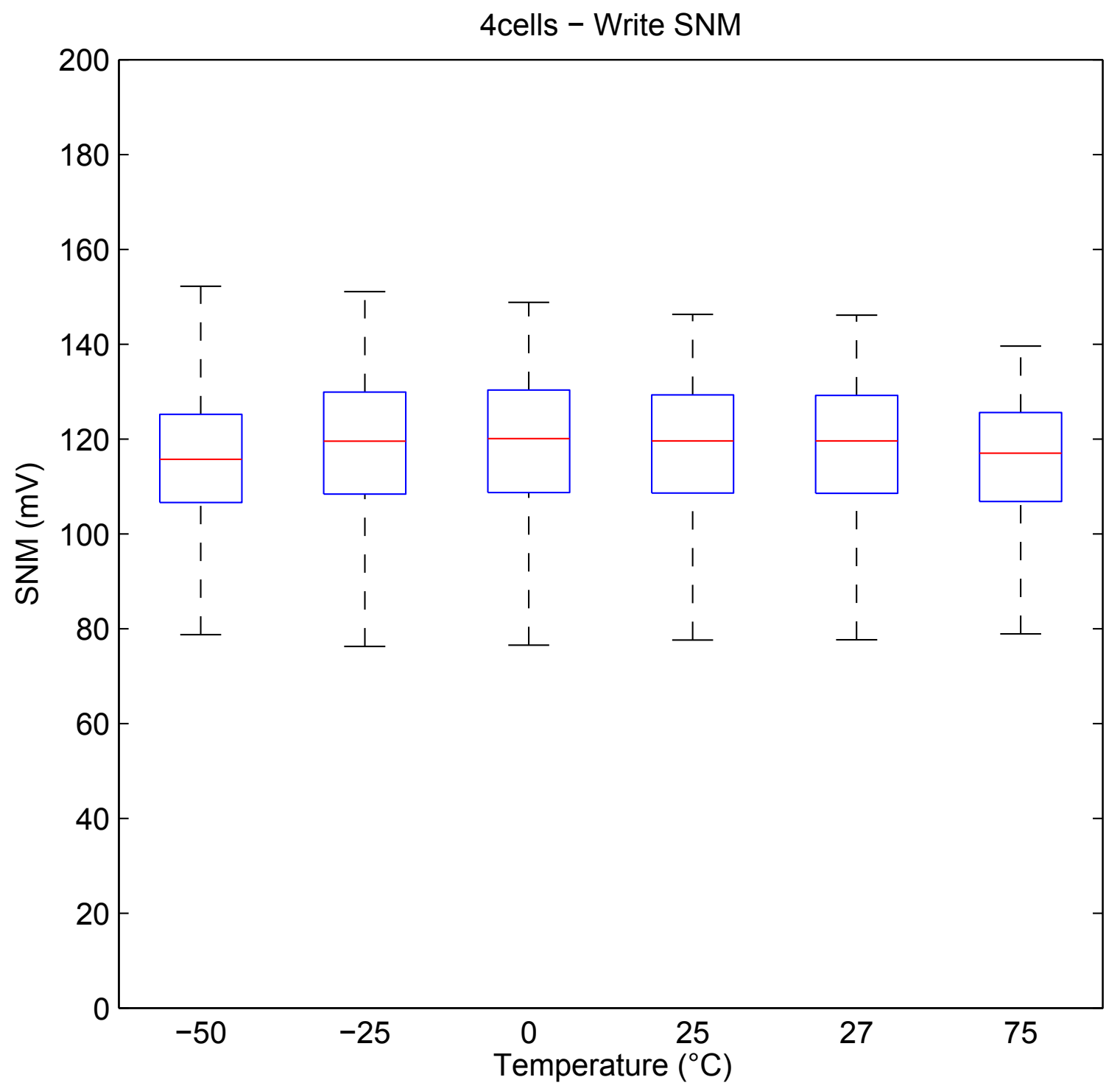

Figure 5.24: Write SNM vs. Temperature $\left({ }^{\circ} \mathrm{C}\right)$ for the 4-cell block. 


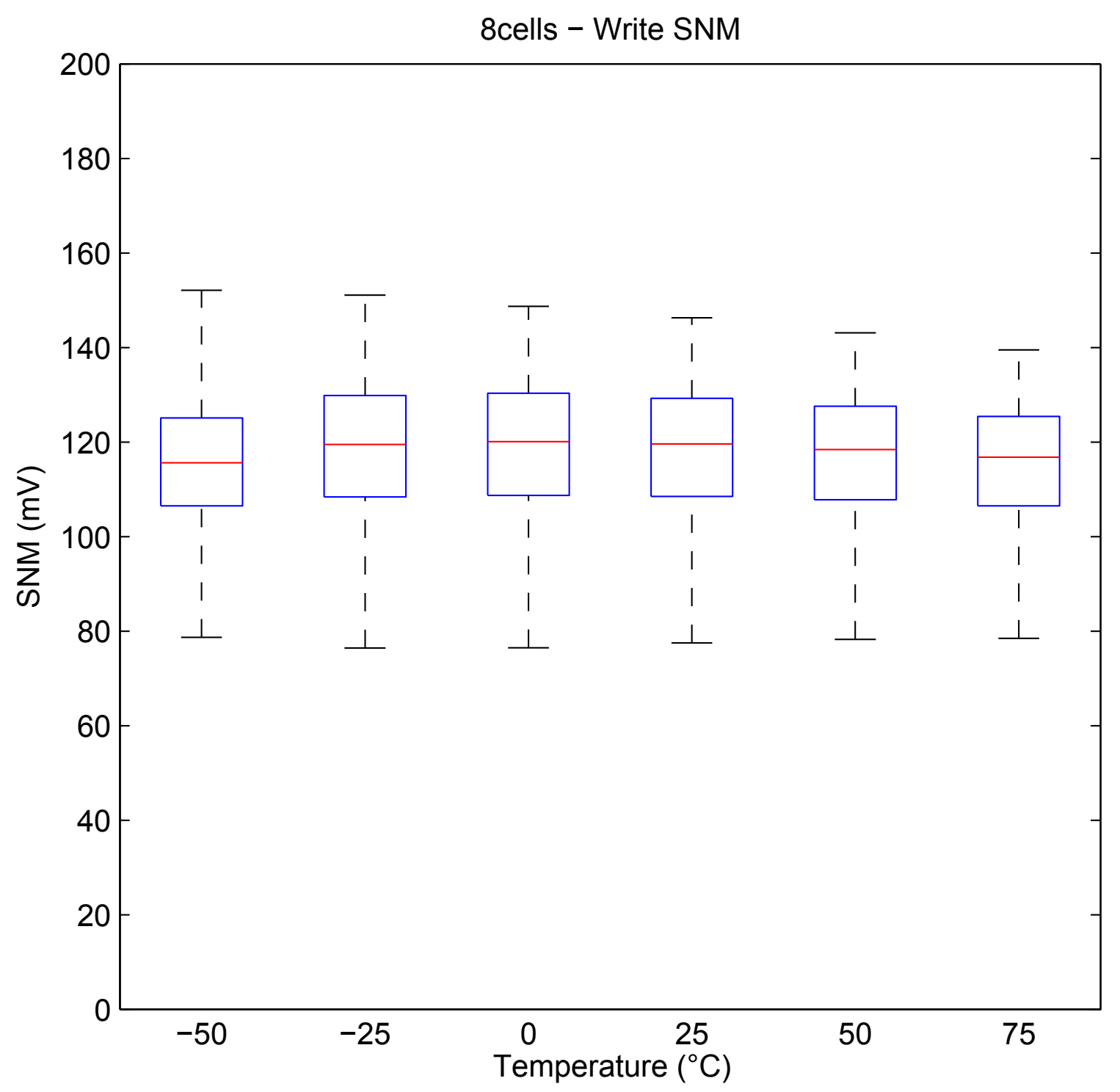

Figure 5.25: Write SNM vs. Temperature $\left({ }^{\circ} \mathrm{C}\right)$ for the 8-cell block. 


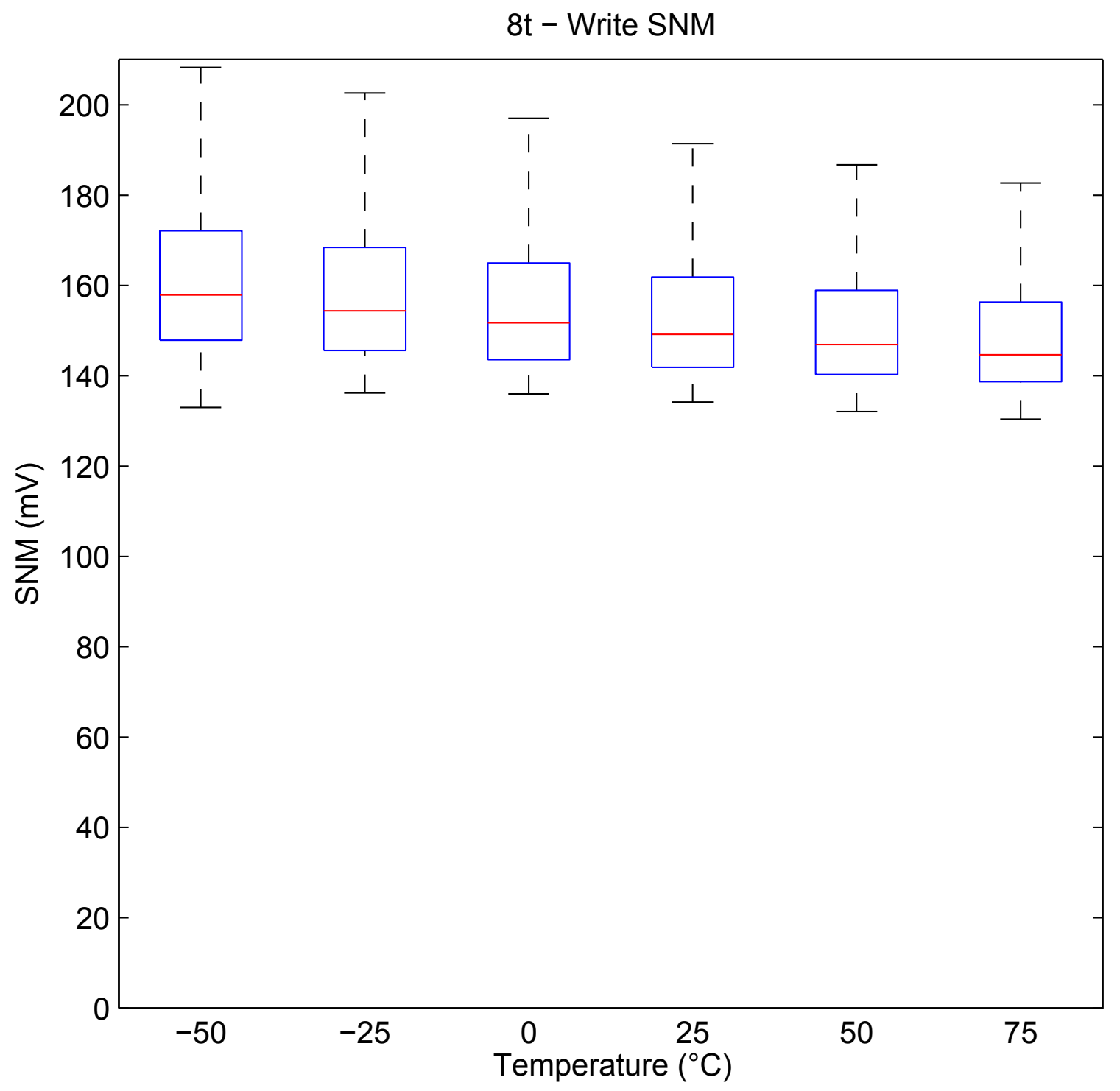

Figure 5.26: Write SNM vs. Temperature $\left({ }^{\circ} \mathrm{C}\right)$ for the traditional $8 \mathrm{~T}$ SRAM cell. 


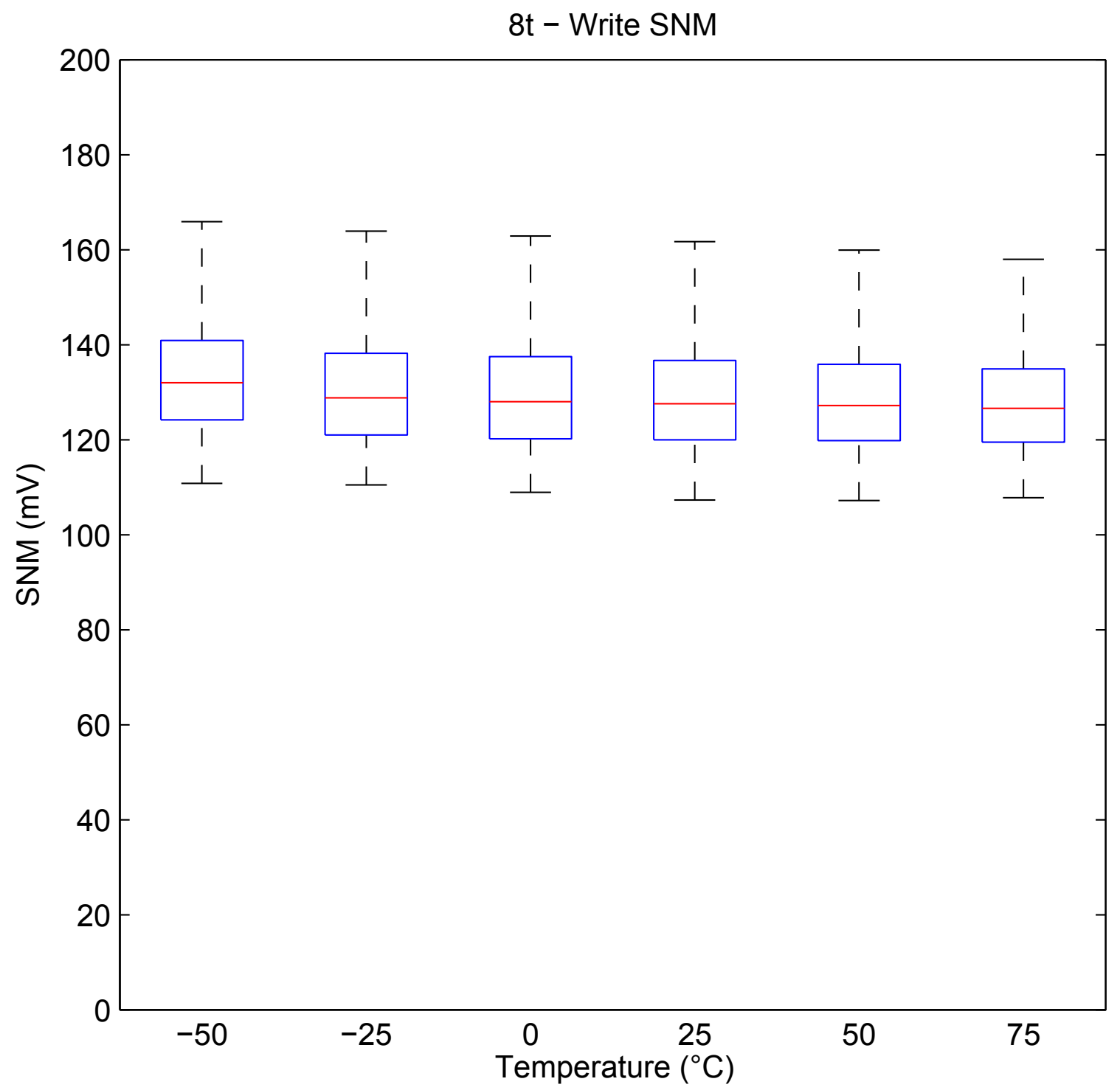

Figure 5.27: Write SNM vs. Temperature $\left({ }^{\circ} \mathrm{C}\right)$ for the single-ended write scheme utilizing the traditional 8T SRAM cell. 


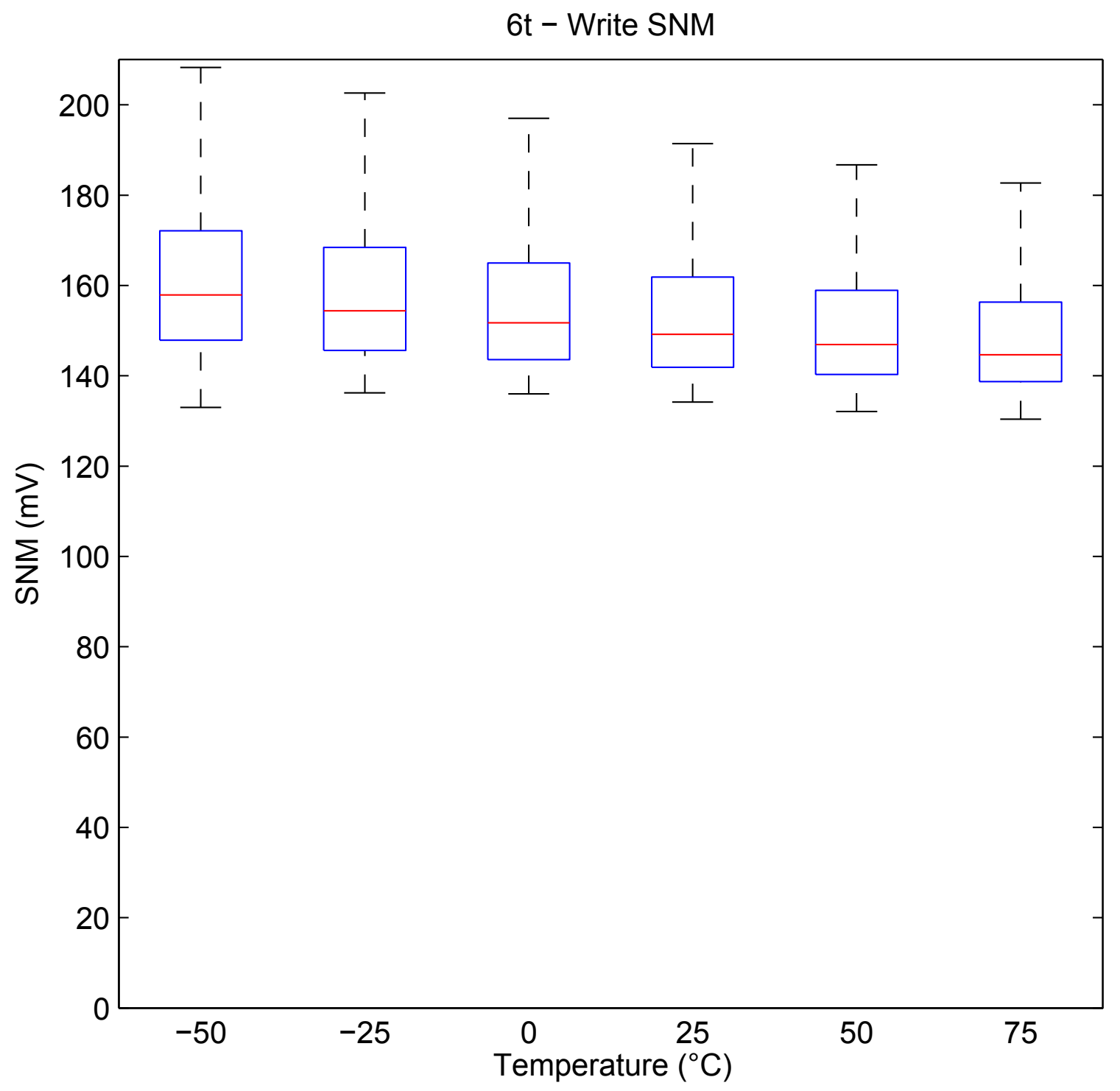

Figure 5.28: Write SNM vs. Temperature $\left({ }^{\circ} \mathrm{C}\right)$ for the traditional $8 \mathrm{~T}$ SRAM cell. 
write operation is the same in both cells, so it seems that the cause for this difference is the use of the write-decoupling transistor inside the cell block.

The proposed design has an almost even spread above and below the median, while the differential and single-ended write operation for the 8T SRAM cells have an increased spread above the median. This shows that the write operation in the SRAM cell blocks are more susceptible to process variations than the traditional $8 \mathrm{~T}$ and standard 6T SRAM cells. The affect of temperature on the SRAM cells is summarized in Figure 5.29.

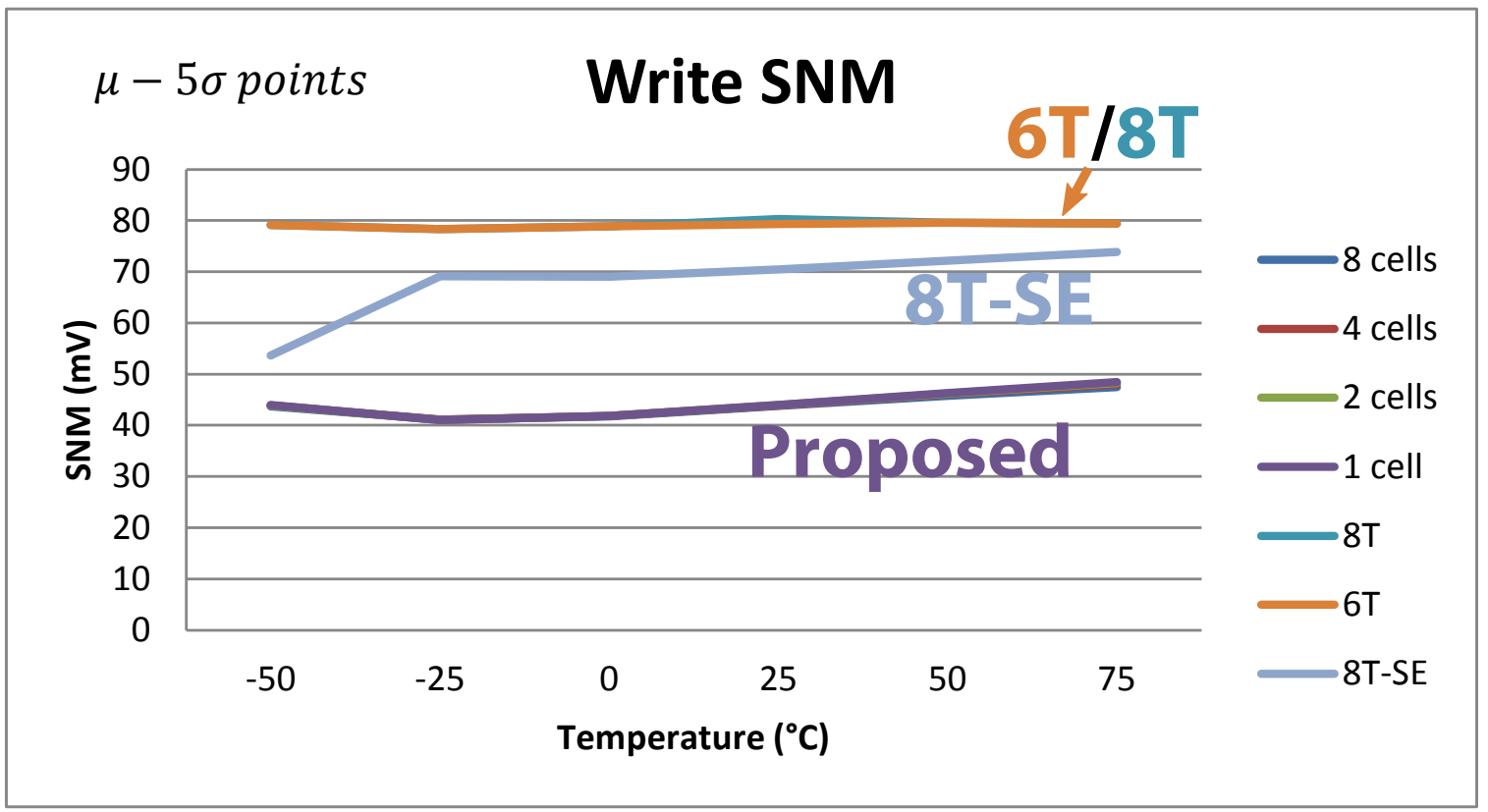

Figure 5.29: Simulation results for Write SNM vs. Temperature $\left({ }^{\circ} \mathrm{C}\right)$ for the different block configurations proposed, the traditional $8 \mathrm{~T}$ cell, the single-ended $8 \mathrm{~T}$ write-scheme, and the standard $6 \mathrm{~T}$ cell. $V_{D D}$ is $300 \mathrm{mV}$. The plotted points are $\mu-5 \sigma$.

Given all these results, the static noise margins of the proposed design is very comparable to the 8T SRAM cell. In many cases it is slightly inferior, but it is definitely an acceptable trade off for the area savings. It is undoubtedly more suitable for sub-threshold operation than the 6T SRAM cell, which has abysmal read SNM. Now that the stability of the cell is proven to be adequate, its performance and power requirements must be examined. 


\section{Chapter 6}

\section{SRAM Block Simulations}

To assess the proposed SRAM architecture more accurately, a complete SRAM array block was implemented, simulated, and compared to a traditional 8T SRAM block. Since the purpose of this study is to design a minimum area SRAM cell, the 8-cell configuration of the proposed design is used. All simulations were done using the worst-case corner, i.e. FS (Fast NMOS, Slow PMOS). The FS corner is the worstcase scenario in terms of SRAM stability, i.e. SNM, because the minimum-size PMOS transistors are inherently weaker than the minimum-size NMOS transistors in the storage cell. It is not necessarily the worst-case for speed or power consumption.

\subsection{SRAM Block}

Figure 6.1 shows the architecture of the implemented 32 kilobit memory block. The array is composed of 16 columns and 256 rows of the 8-cell block configuration, which translates to an array of 128 x 256 bits. To be homologous with modern computers, the block was constructed to be byte-addressable. Thus, there is an address space of $2^{12}$, or 4096, 8-bit words.

The structure of the block was designed to support a bit-interleaving structure; the architecture writes concurrently into one cell in multiple cell blocks contained in the same row. The bits are interleaved between the blocks. The following shows the composition of a row, where $\mathrm{A}$ is the first byte, and I is the second byte:

$$
\left|A_{1}\right| I_{1}\left|A_{2}\right| I_{2}\left|A_{3}\right| I_{3}\left|A_{4}\right| I_{4}\left|A_{5}\right| I_{5}\left|A_{6}\right| I_{6}\left|A_{7}\right| I_{7}\left|A_{8}\right| I_{8} \mid
$$

As such, 8 out of 16 columns are read-from or written-to at the same time. The 
Table 6.1: Byte Layout

\begin{tabular}{|c|c|c|c|c|c|c|c|}
\hline Col 1 & Col 2 & Col 3 & $\ldots$ & Col 16 & Col 17 & $\ldots$ & Col 128 \\
\hdashline$A_{1}$ & $B_{1}$ & $C_{1}$ & $\ldots$ & $A_{2}$ & $B_{2}$ & $\ldots$ & $P_{8}$ \\
\hline
\end{tabular}

composition of the data inside a cell block would be:

$$
\left|A_{x}\right| B_{x}\left|C_{x}\right| D_{x}\left|E_{x}\right| F_{x}\left|G_{x}\right| H_{x} \mid,
$$

where $\mathrm{A}, \mathrm{B}, \mathrm{C}, \mathrm{D}, \mathrm{E}, \mathrm{F}, \mathrm{G}$, and $\mathrm{H}$ are all separate bytes, and $x$ is an arbitrary position in the byte. The value of $x$ is the same in all the bits stored in the block. For example, the block storing $A_{1}$ in 6.1 , would have a composition of:

$$
\text { | } A_{1}\left|B_{1}\right| C_{1}\left|D_{1}\right| E_{1}\left|F_{1}\right| G_{1}\left|H_{1}\right| \text {, }
$$

and the block storing $I_{1}$ would have a composition of:

$$
\text { | } I_{1}|J 1| K_{1}\left|L_{1}\right| M_{1}\left|N_{1}\right| O_{1}\left|P_{1}\right| \text {. }
$$

In this example, $\mathrm{A}$ is the first byte in the row, $\mathrm{B}$ the second, etc, with $\mathrm{P}$ being the sixteenth.

The address decoder used is a simple clock-based decoder with a predecoding stage designed for maximum integration density. It is not optimized for speed or minimum logical effort. The data inputs for the block are connected to the word-line demultiplexers. Each bit input is shared between two demultiplexers (i.e. word-line demultiplexers 1 and 2 are connected to 'Data In [1]'). The read bit-line multiplexers selects between two global read bit-lines. The sense amplifiers utilized are standard high-skewed inverters, which are arguably ideal for sub-threshold large-single sensing single-ended designs.

The conventional 8T SRAM array block is shown in Figure 6.2. The array is composed of 128 columns and 256 rows of the $8 \mathrm{~T}$ cell, which translates to an array of $128 \times 256$ bits. Again, there is an address space of $2^{12}$, or 4096, 8-bit words.

Bit-interleaving was implemented in the same manner as the proposed architecture for the $8 \mathrm{~T}$ array block. One bit in a byte is written every 16 bits in a row. This is 


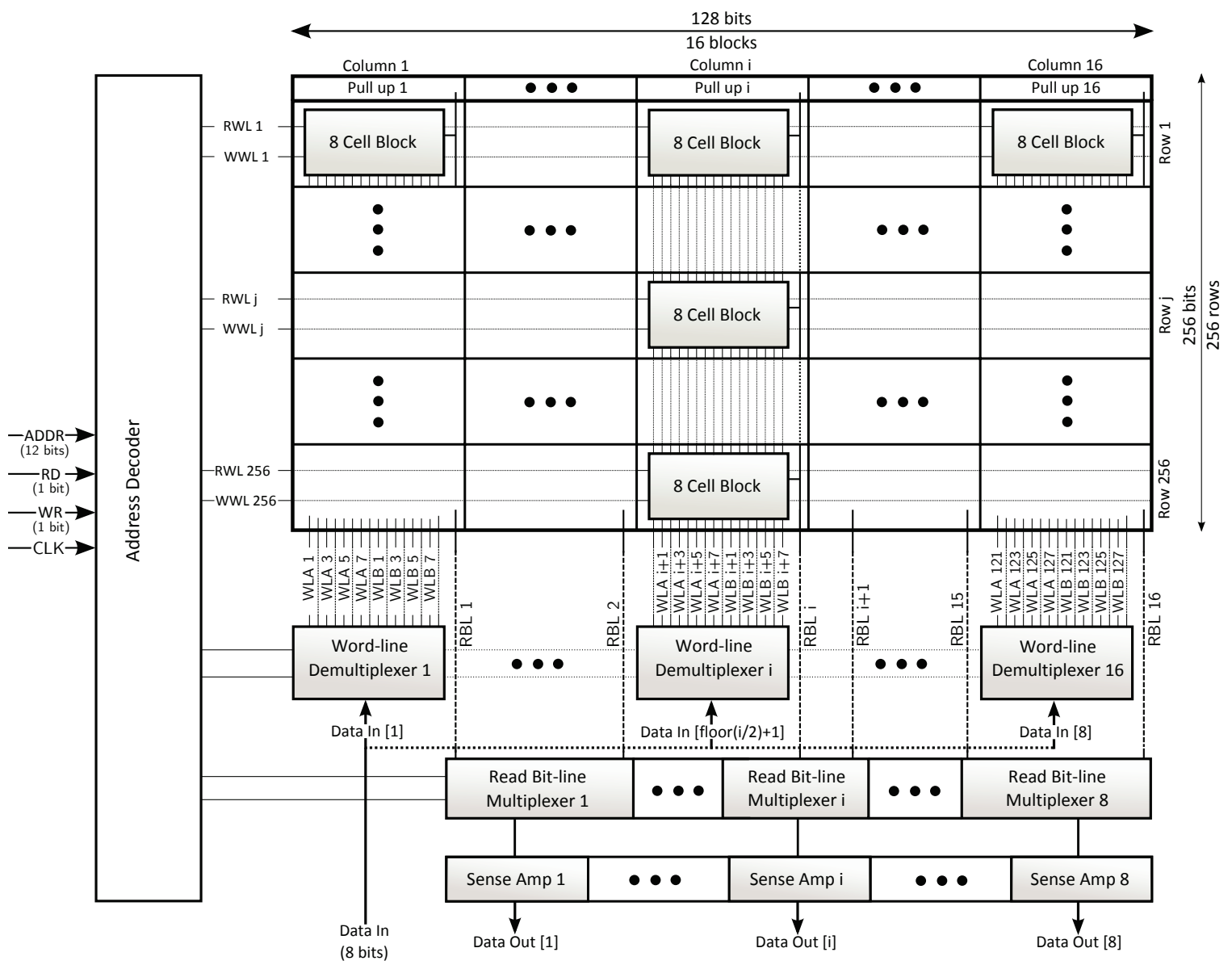

Figure 6.1: Array level block diagram of the $32 \mathrm{~kb}$ memory block for the proposed SRAM architecture.

perhaps best explained by Table 6.1, which shows the layout of bytes in a row. In Table 6.1, A is the first byte in the row, B the second, etc, with $\mathrm{P}$ being the sixteenth.

With the exception of the composition of the array, the architecture is very similar to the proposed design block. The main difference is the use of a write driver for the write bit-lines and pull-ups needed for all bit-lines. In the proposed design, the wordlines WLA and WLB are brought high when selected, but are low when not. In the $8 \mathrm{~T}$ cell, the write bit-lines are pre-charged high, but then are selectively discharged depending on the value being written. Despite these differences in operation, the schematics of the write driver and word-line multiplexer are very similar, with the biggest difference being the additional circuitry in the word-line multiplexer to handle read selection as well. 
Since all bit-lines are required to pre-charged, strong pull-ups are needed for the write bit-lines in addition to the read bit-lines. Due to the differences in architectures, there are 384 bit-lines in the 8T block, while only 16 global bit-lines in the proposed block. Another, albeit relatively minor, difference is that the read bit-line multiplexer in the $8 \mathrm{~T}$ array block is connected to 16 bit-lines instead of 2 like in the proposed architecture.

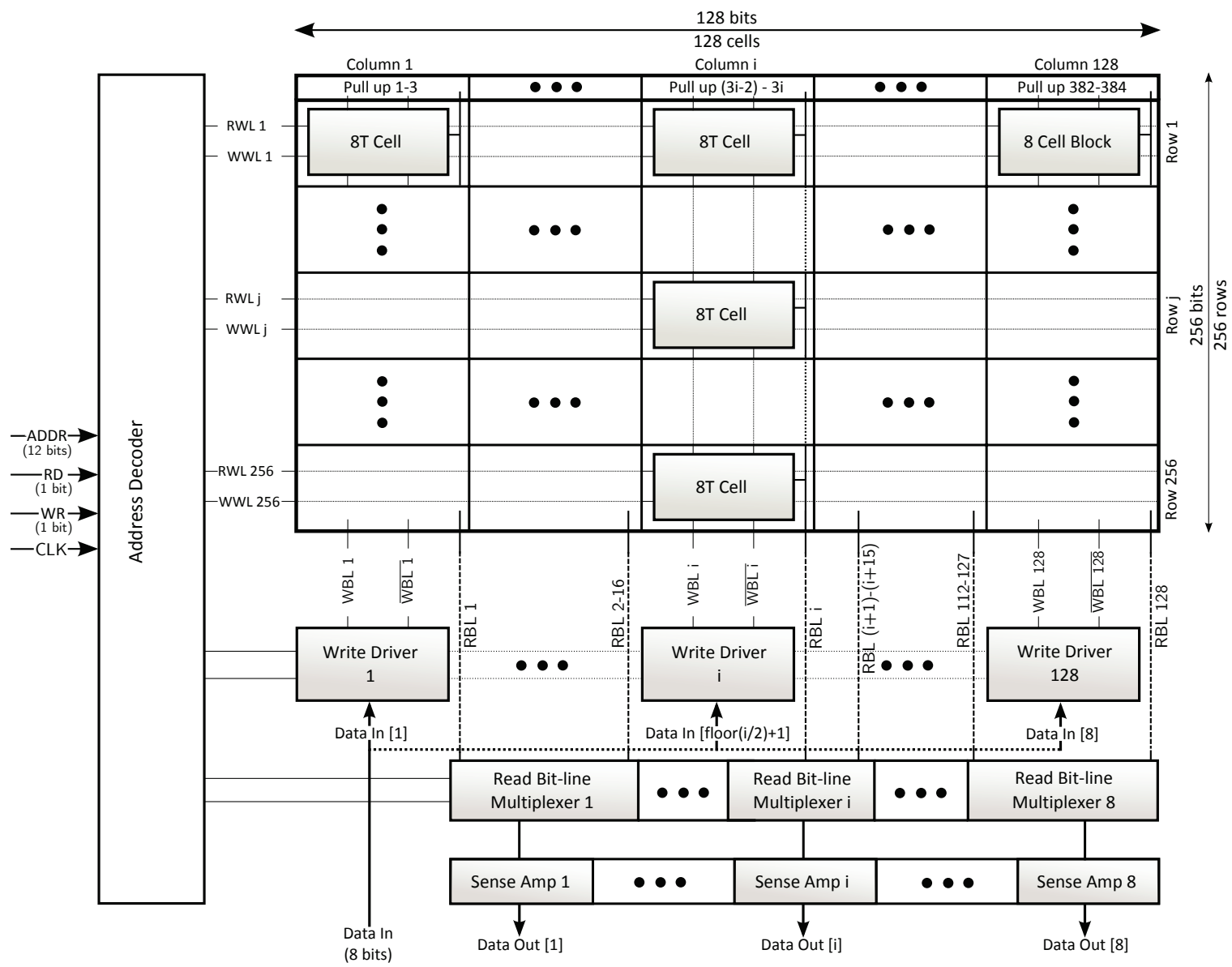

Figure 6.2: Array level block diagram of the $32 \mathrm{~kb}$ memory block for the traditional 8T SRAM cell.

\subsection{SRAM Block Operation}

While the architecture of the SRAM blocks are very similar, the write operations of each block is fairly different. This is verified by comparing the signals in Figure 6.5, 
for the proposed design, and Figure 6.3, for the 8T block. In the 8T SRAM block, the write operation starts when the WR signal is enabled and CLK (the clock) is high. As soon as CLK is high, the pull-ups for the bit-lines are disable. This causes the write bit-lines to steadily drop due to bit-line leakage. However, once the decoder processes the address and write/read signals, the write driver further pulls down the appropriate write bit-line for the desired written data ( $\overline{W B L}$ in Figure 6.3), while leaving the other write bit-line floating.

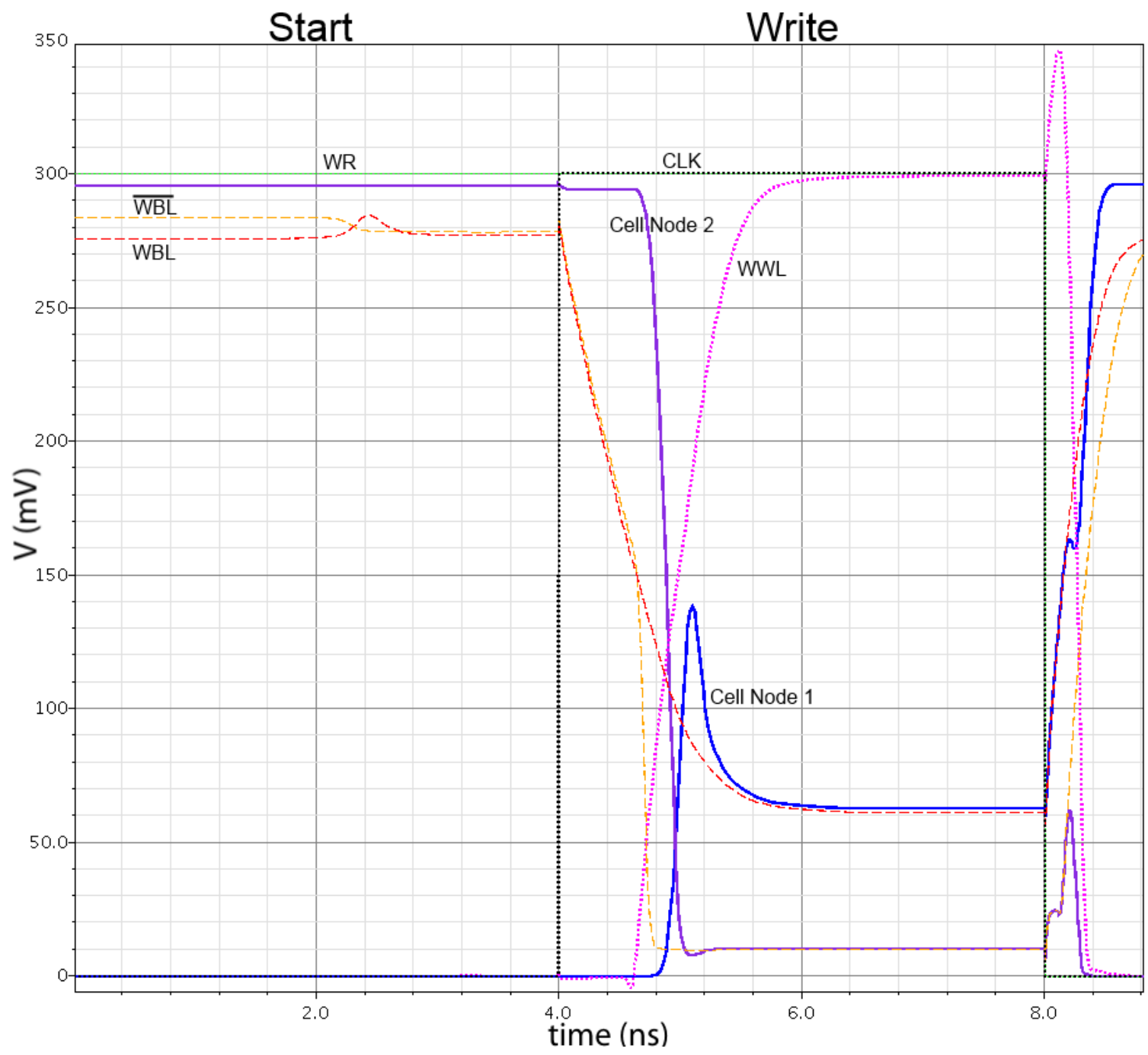

Figure 6.3: Simulated signals involved in the write operation of the $8 \mathrm{~T}$ block for FS corner at $27^{\circ} \mathrm{C}$.

Slightly before the write driver is activated, the decoder enables the WWL (write 
word-line) signal for the selected row, which allows for the modification of the data stored in the selected cell. The write bit-line that has been discharged sets the value of the connected cell node to logic ' 0 '. Feedback through the back-to-back inverters then raises the voltage of the other node. In Figure 6.3, $\overline{W B L}$ discharges Cell Node 2 that, subsequently, raises the voltage of Cell Node 1.

It is evident that the voltage of Cell Node 1 never becomes logic level ' 1 ' due to it being connected to the high capacitance write bit-line. However, once the write operation is completed and the SRAM block is in the idle state, feedback by the cross-coupled inverters brings the nodes to their appropriate level of $V_{D D}$ and GND. Figure 6.3 also shows that it takes some time after the clock switches low for all relevant signals to be disabled, allowing the SRAM block to go into the idle state. This is due to the decoder taking time to process the switching clock signal. This delay is present in all operations.

In the 8T SRAM block architecture used for comparison in this study, the worstcase leakage in the write bit-lines can severely affect the write ability of a cell. The worst-case leakage in the write bit-line occurs when all cells in a column are storing the same value. The write bit-line connected to the node storing '0' will suffer from a relatively substantial amount of leakage. If it is desired to change the value of one cell in this column, the write bit-line connected to the node storing a ' 1 ' must be discharged to change the node's value. This presents a race condition on which can discharge the bit-line faster, the write driver or the cumulative leakage.

Figure 6.4 presents this problem when running a simulation in the worst-case FS corner. It can be seen that there is competition between WBL and $\overline{W B L}$. Once the pull-ups are disabled, $\overline{W B L}$ steadily decreases due to leakage. When the write driver is enabled, it quickly pulls down WBL and is able to discharge the desired bit-line slightly faster than the leakage on its counterpart, $\overline{W B L}$. However, this results in a marginal difference between the two nodes and bit-lines. Since a cell is written to by discharging one node, in Figure 6.4 the difference is enough to recover the desired data values after the end of the write operation. This issue can be mitigated by implementing a faster decoder, a stronger write driver, or by having the write driver keep the other node high. Although, all of these would result in a greater area consumption and/or increase in power consumption.

The write operation of the proposed SRAM block was described in Chapter 4. The decoder processes the input, setting WWL high and enabling the proper select 


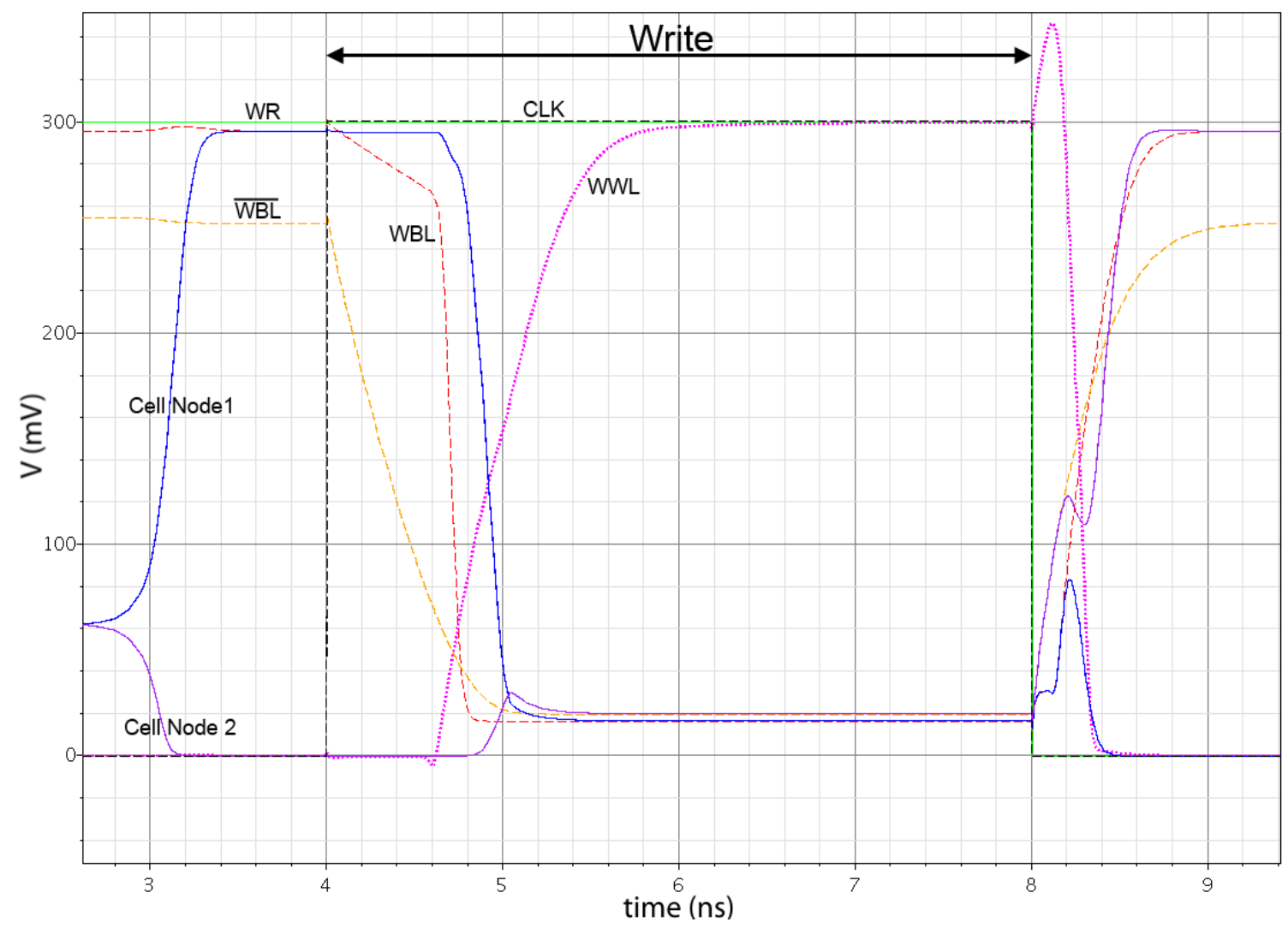

Figure 6.4: The problem with the write operation in the $8 \mathrm{~T}$ block. Simulation performed for $\mathrm{FS}$ corner at $27^{\circ} \mathrm{C}$.

world-line (WLA or WLB). Setting WWL high brings the local bit-line (LBL) in the selected block to GND. Once the proper word-line is selected, a node in the selected cell is exposed to the local bit-line, setting its value to ' 0 '. Feedback by the crosscoupled inverters then sets the opposite node to ' 1 '. This is illustrated in Figure 6.5. In this example, WLA is selected, forcing Cell Node 2 to ' 0 ' that, in turn, sets Cell Node 1 to ' 1 '.

Figure 6.5 shows that the node being set to ' 1 ' is actually set to a logic ' 1 ' during the write operation, unlike in the $8 \mathrm{~T}$ SRAM block. This is the result of the node remaining isolated during the operation. However, the value of the node doesn't reach $V_{D D}$ until after then write operation is over. This is due to the leakage through the access transistor as a result of the local bit-line is connected to GND during the write operation. After the write operation, this node does initially decrease in voltage as a result of RWL going low before WLA, but, after WLA is low and the access transistors 
are $\mathrm{OFF}$, feedback forces the node to $V_{D D}$. The initial decrease can be avoided by ensuring WLA goes low before WWL. After the write operation, the local bit-line is left floating, reducing the access transistor leakage. There isn't a race condition in the write bit-lines like in the $8 \mathrm{~T}$ SRAM block since there aren't any write bit-lines. Nor are any bit-lines left floating during the operation, so they cannot be affected by leakage. There is still delay caused by the decoder once the write operation is over, however.

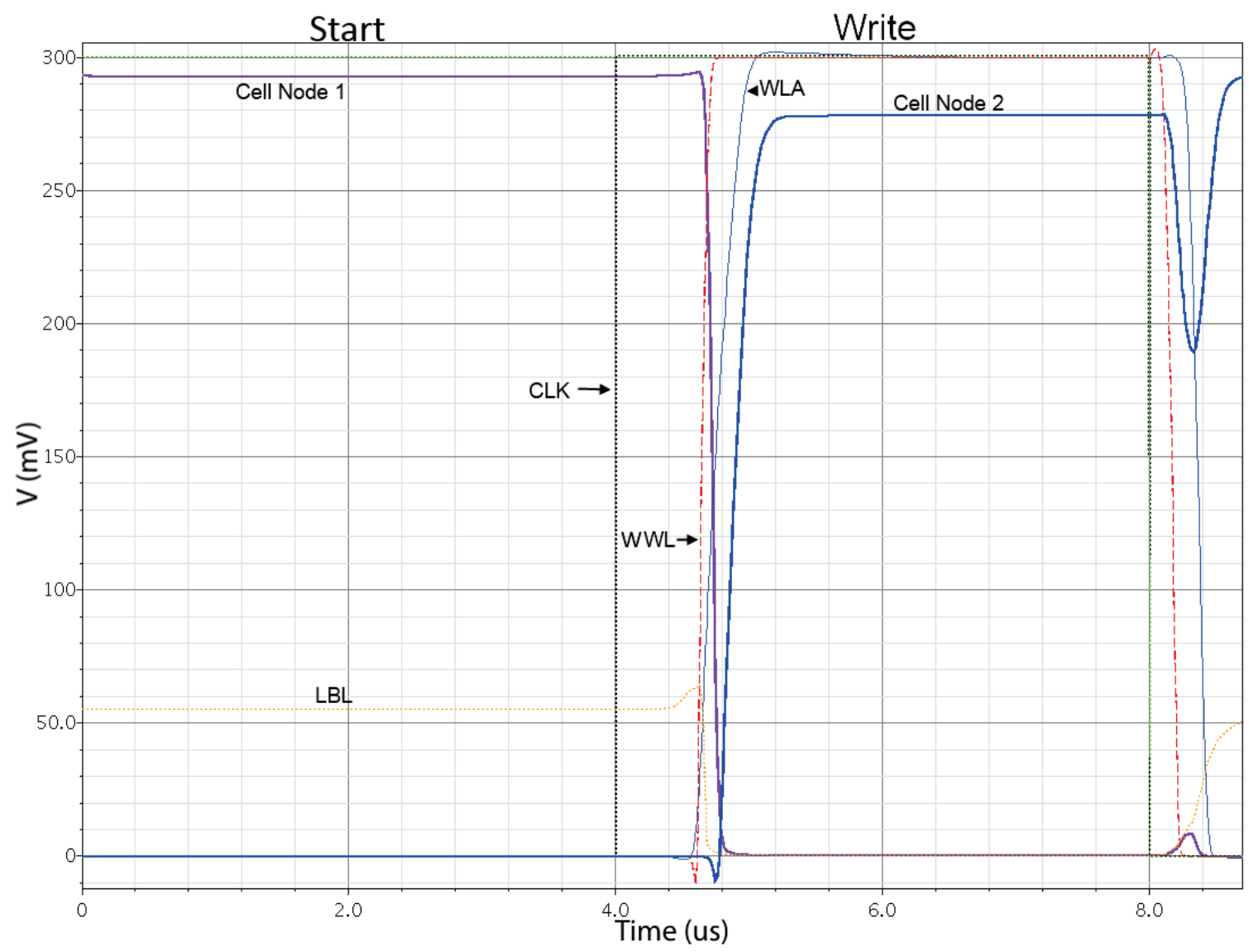

Figure 6.5: The signals involved the write operation in the proposed block. Simulation performed for FS corner at $27^{\circ} \mathrm{C}$.

Conversely to the write operation, the read operation is fairly similar in both SRAM blocks. In the $8 \mathrm{~T}$ SRAM block, the read operation occurs when the decoder enables the RWL (read word-line) signal for the selected row. This enables the read access transistor in all 8T cells in the selected row. When the stored value in a cell 
is ' 1 ', the read decoupling transistor in the cell is also enabled. This results in the drainage of the read bit-line (RBL). The multiplexer then selects the desired bit for read, sending it to the sense amplifier, which reads the value. This operation is shown in Figure 6.6. Note that the reason for the rise of the RWL signal at the beginning of the read operation is due to the slowness of the decoder.

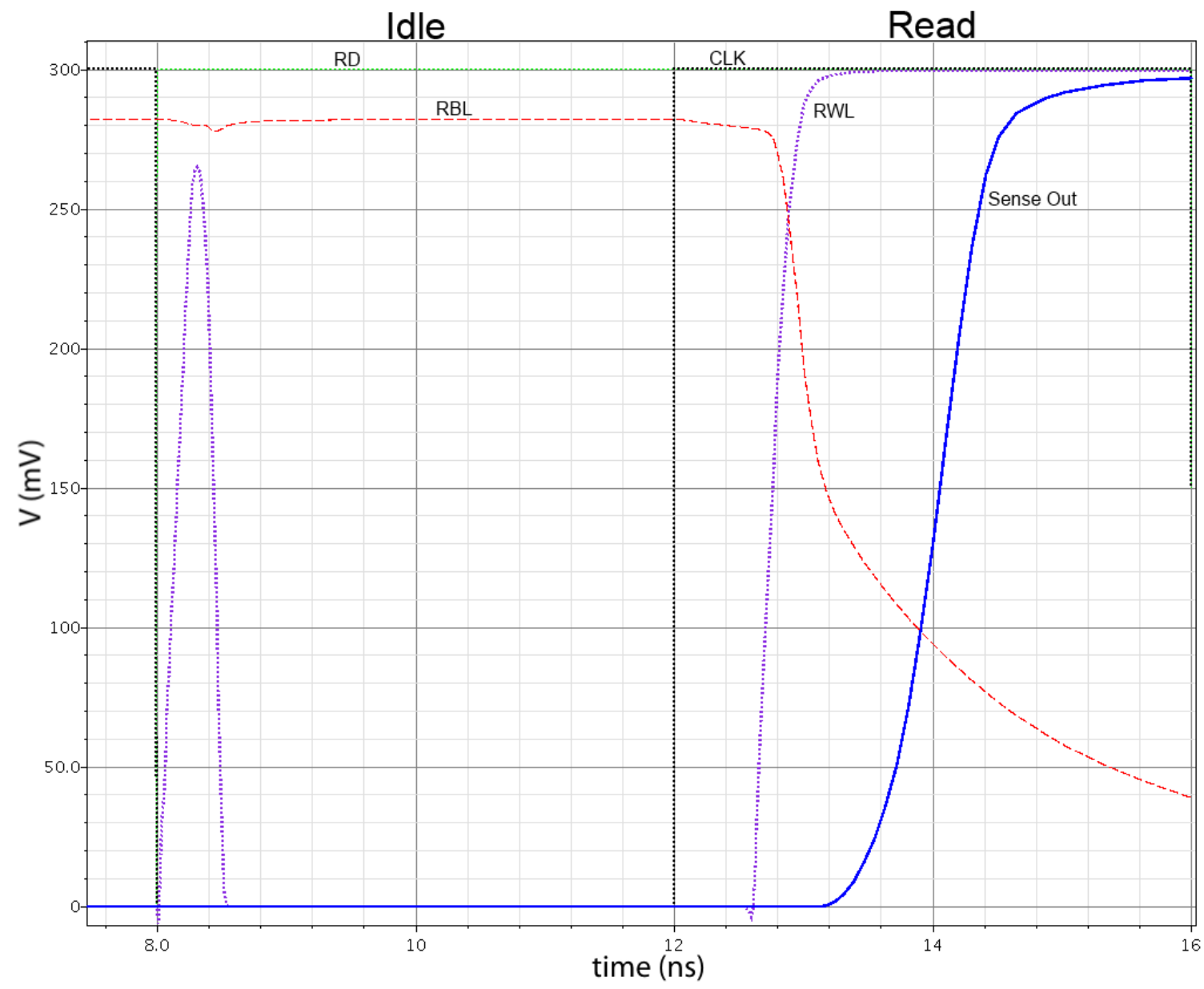

Figure 6.6: The signals involved the read operation in the $8 \mathrm{~T}$ block. Simulation performed for FS corner at $27^{\circ} \mathrm{C}$. Note that the reason for the spike of the RWL signal in the beginning of the read operation is due to the slowness of the decoder.

The read operation of the proposed block similarly occurs when the decoder enabled the RWL signal for the selected row, which enables the read access transistors in all cell blocks in that row. At the same time, the word-line demultiplexer selects 
the desired column by enabling the WLB signal. The WLB signal enables one access transistor in every cell in the selected row. When reading a ' 1 ', the enabled access transistor connects the selected storage cell to LBL, setting LBL to ' 1 ' and enabling the read decoupling transistors for that block, draining RBL. The read bit-line multiplexer then sends the selected column to the sense amplifier, which reads the value. This operation is illustrated in Figure 6.7. Again, the signals begin to go high at the beginning of the idle state as a result of changing the signals at the same time as the clock switches low. It takes time for the decoder to process the low clock state.

If the read bit-lines between both blocks are compared, it is evident that RBL in the $8 \mathrm{~T}$ block drains faster once RWL is asserted due to the read decoupling transistor in the selected cell being fully $\mathrm{ON}$ with a value of $V_{D D}$ being applied to the gate. As RBL drains more and more, the drain current slowly decreases due to $V_{D S}$ decreasing in the read access transistor. However, by this point, the sense amplifier has already started to go high, making the decreasing drain current trivial. RBL in the proposed block drains much slower due to the read decoupling transistors not being fully ON. The drain current also slowly decreases as RBL is drained, but the change is not as drastic as the drain current is already impaired. RBL does go to GND faster in the proposed design, but this is inconsequential, as the high-skewed sense amplifier has already read the value by then.

The Idle state in both blocks is achieved by disabling all control signals and precharging all bit-lines. In the $8 \mathrm{~T}$ SRAM block this means that all write bit-lines (WBL and $\overline{W B L}$ ) and read bit-lines (RBL) are pre-charged and kept high in the idle state. Only RBL is pre-charged and kept high in the proposed block. It is necessary to put the blocks in the idle state between operation so the bit-lines can pre-charge. Since all control signals are disabled, the local bit-lines in all the blocks are left floating in the idle state. This causes the local bit-line to raise slightly in voltage as it reaches an equilibrium in regards to leakage, as seen in Figure 6.7.

\subsection{Comparison to 8T SRAM Block}

In order to compare the proposed SRAM block to the 8T SRAM block, data was written to the same byte in both blocks and then read out. The basic test done was to write 01010101 (i.e. 85 in decimal) to register 00010000 (16), and then see if the data was read back successfully. Both blocks completed the test successfully with 


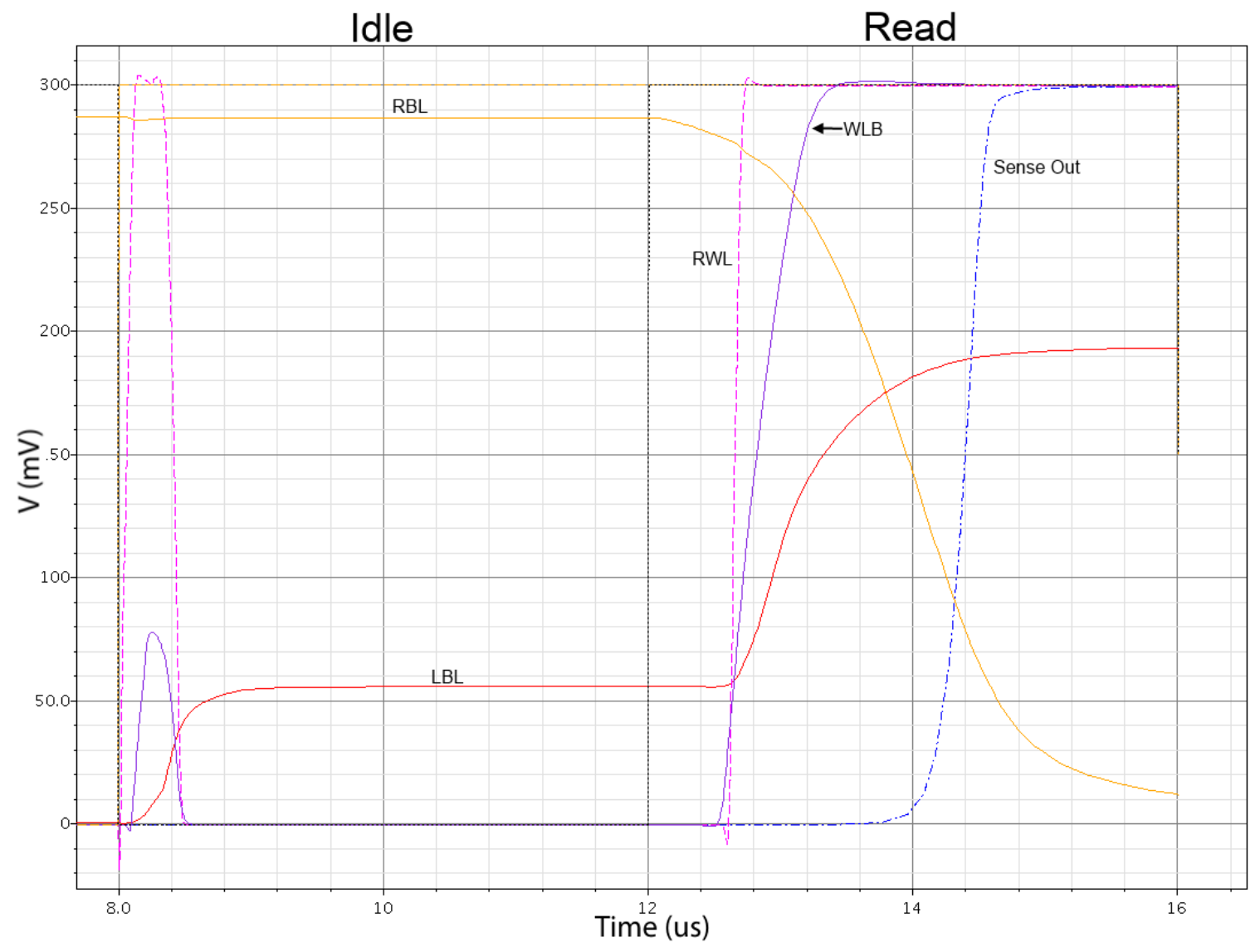

Figure 6.7: The signals involved the read operation in the proposed block. Simulation performed for FS corner at $27^{\circ} \mathrm{C}$. Note that the spikes in the signals in the beginning of the idle state is due to changing the signals at the same time as the clock switches low. It takes time for the decoder to process the low clock state.

a $V_{D D}$ of $300 \mathrm{mV}$, with a time period of $4 \mu \mathrm{s}$ for the clock (to account for the slow read operation). The results of this simulation are shown in Figure 6.3, Figure 6.5, Figure 6.6, and Figure 6.7. The first period of operation in the write diagrams is when the block has just started up, so the values stored in the cells are of random arbitrary value.

Since this test encompasses all operation cases, it was also used to measure the power and energy consumption. Figure 6.8 shows the power consumption of both blocks throughout all states. The power consumption in the start cycle is inconsequential since none of the bits are initialized and many nodes in the storage cells are 


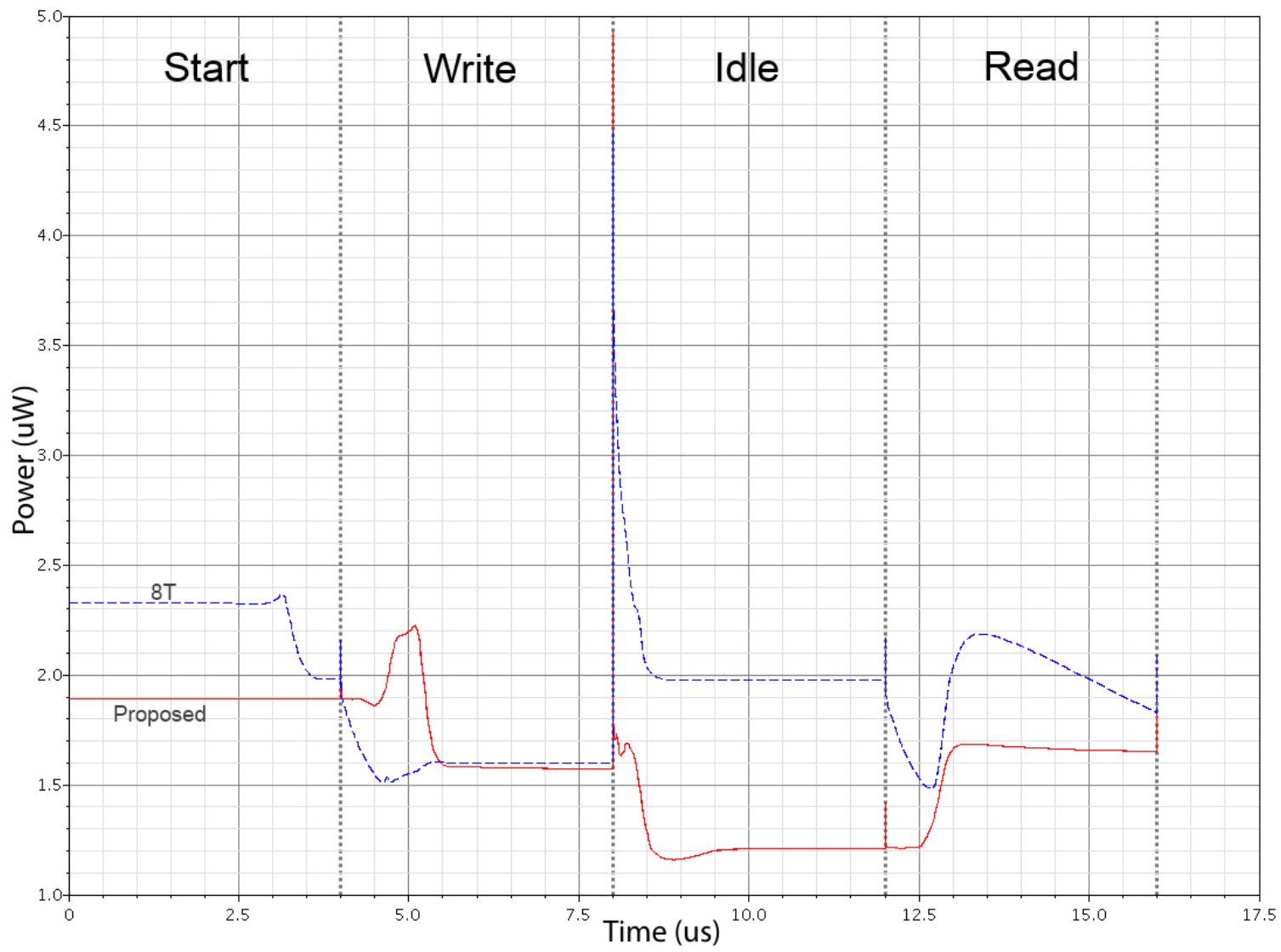

Figure 6.8: Comparison of the power consumption during the start-up, write, idle, and read operations.

in an unstable state (resulting in increased leakage). Note that there is a sudden drop in the power consumption in the $8 \mathrm{~T}$ block near the end of the start cycle. At this point, before any signals have been enabled or changed, the unstable states inside the storage cells have reached a state of stability. The storage cells in the proposed block do not reach a point of stability before the end of the start cycle. This is because both sides of the SRAM cells are connected to the same local bit-line, whereas each side of the 8T SRAM cell is connected to a different bit-line. The unequal amount of leakage into each node in the $8 \mathrm{~T}$ cell forces the nodes to a state of stability through feedback. 


\subsubsection{Write Operation}

By analyzing the power consumption of the SRAM blocks in Figure 6.9, the power consumption of writing one byte into each SRAM block can be found. Looking at the $8 \mathrm{~T}$ block, it is apparent that the power consumption drops as the pull-ups for the bit-lines are turned off and slowly discharge. Then, as WWL is enabled, which turns on the access transistors in the selected row, there is a spike in the power consumption followed by a steady increase as the values in the storage cell are written to. Once the values are written to the cells, the power consumption reaches a steady state. The average power consumption over the $4.0 \mu$ s period is $1.601 \mu \mathrm{W}$. Figure 6.10 shows the total energy consumed during the write operation, which is $6.39 \mathrm{pJ}$.

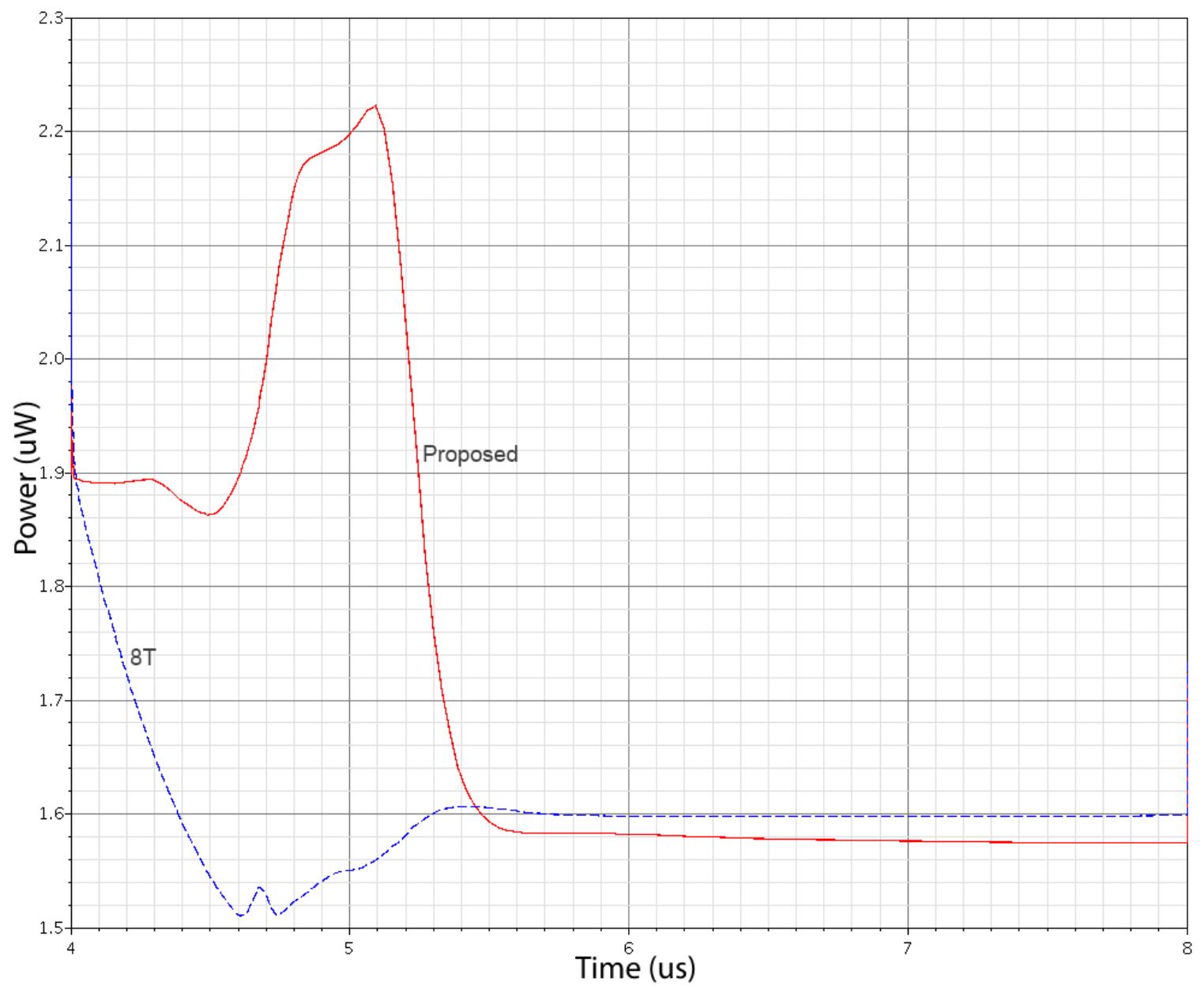

Figure 6.9: Comparison of the power consumption during a write. Close up view of Figure 6.8. 


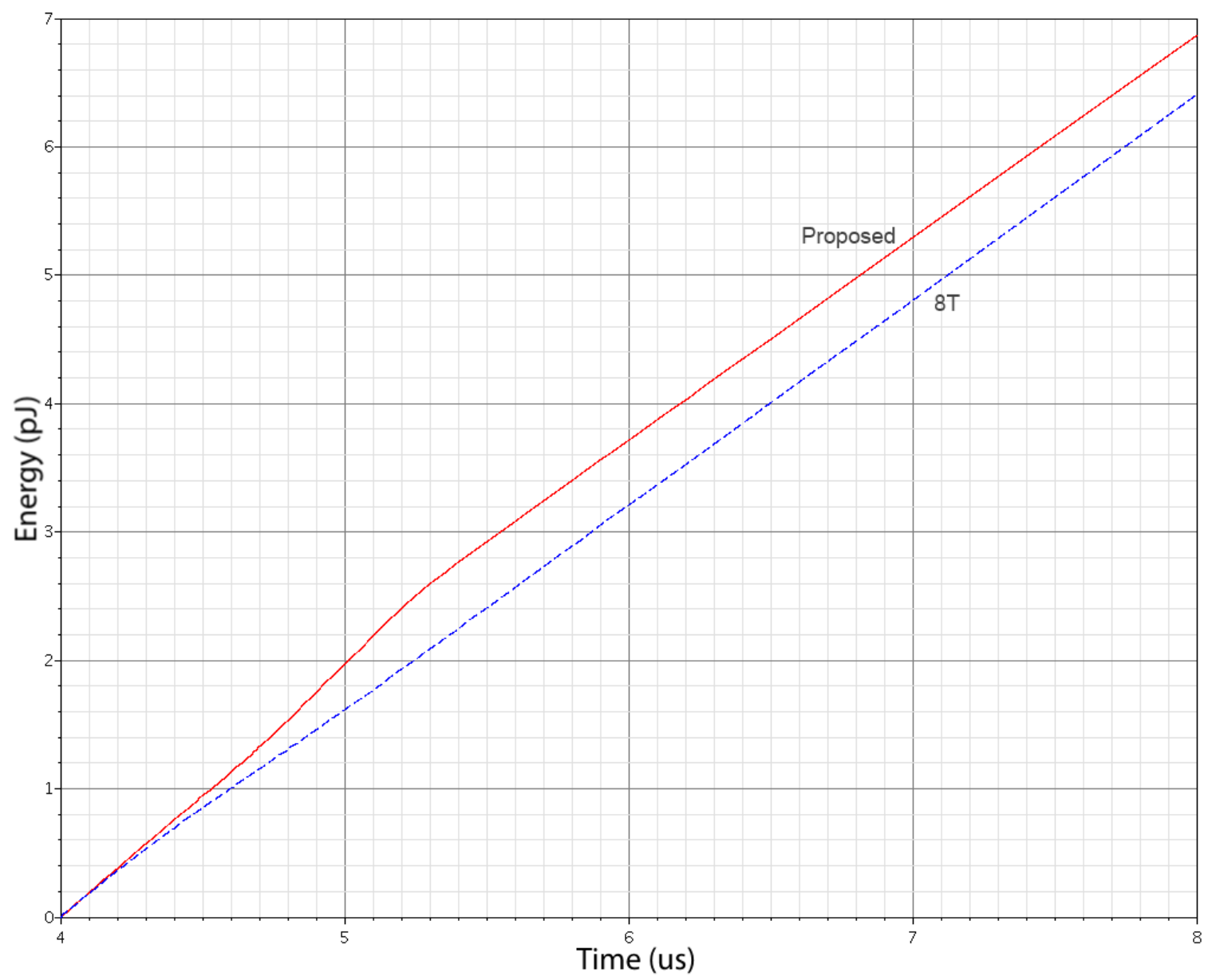

Figure 6.10: Comparison of the energy consumed during a write.

The power consumption of the proposed design remains relatively constant at the start. Once the decoder has processed the high clock signal, there is an increase in power consumption as a result of enabling WWL and WLA/WLB, which turn on the write decoupling transistors in the selected row and the access transistors in the selected column, respectively. Once the values are written to the bit cells, the power consumption begins to drop until the storage cells reach a steady state. The power consumption is quite large in this case because there are numerous cells that are not in a steady state until the access transistors have been turned on, forcing them into a steady state through feedback. A such, this test is not an accurate measure of the power consumption when writing a single byte in the block. Nevertheless, the average power consumption over the $4.0 \mu$ s period is $1.717 \mu \mathrm{W}$. Figure 6.10 shows the total energy consumed during this write operation is $6.87 \mathrm{pJ}$. 
For a more accurate portrayal of the power consumption during the write operation, another test was done. In this test 01010101 (85 in decimal) was written to register 00010000 (16), then in the next operation, 10101010 (170 in decimal) was written to the same register. Therefore, this test allows for the measurement of the amount of power required to switch all the bits in a byte, where half the bits are 1s. The power consumption for the second operation in this test is shown in Figure 6.11.

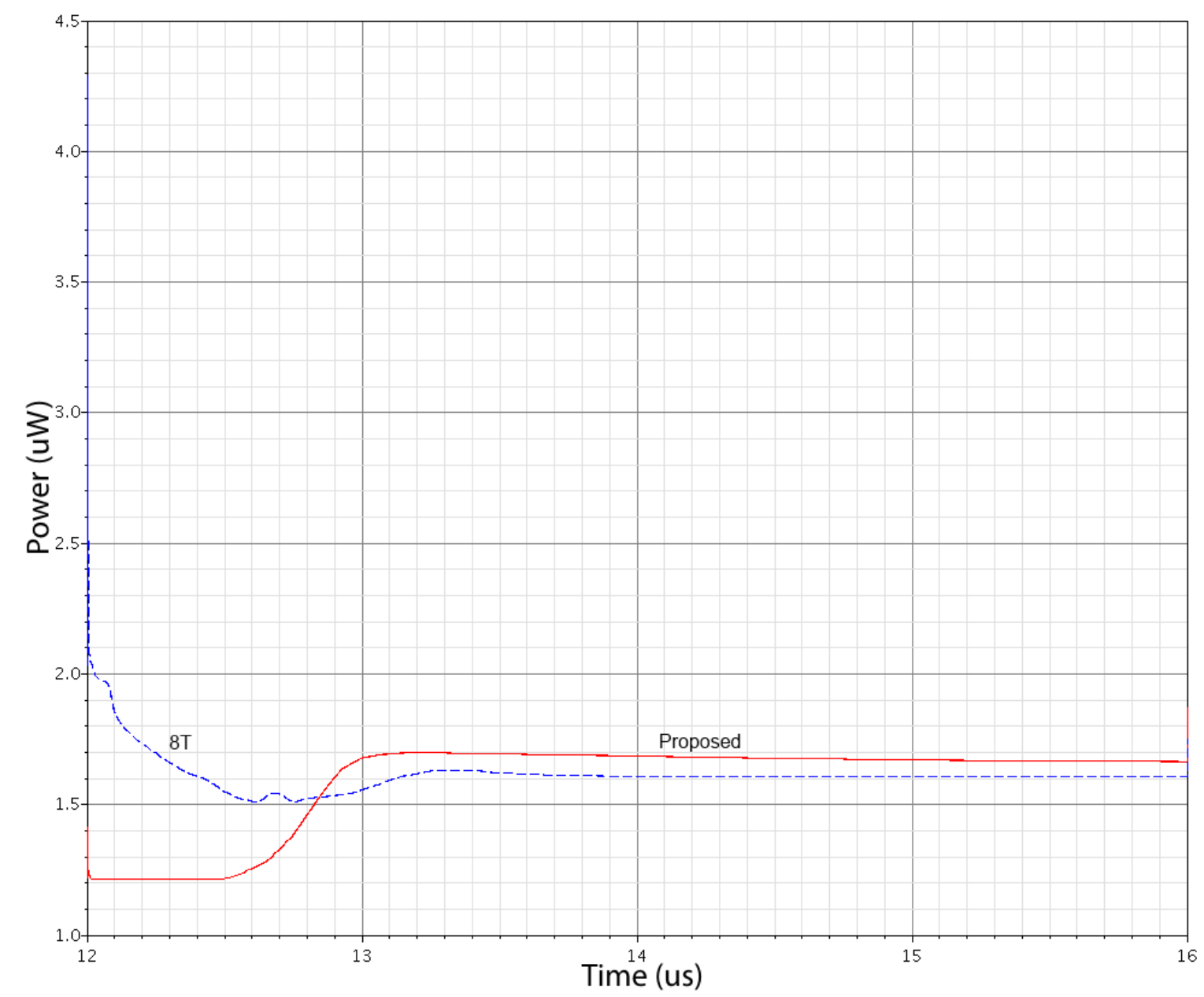

Figure 6.11: More accurate comparison of the power consumption during a write.

The power consumption of the $8 \mathrm{~T}$ write operation is very similar to the power consumption in the original test because, in both cases, all the bits in the block have reached a steady state before the operation began. Conversely, the proposed block has a lower power consumption than in the original test. There is a steady increase in 


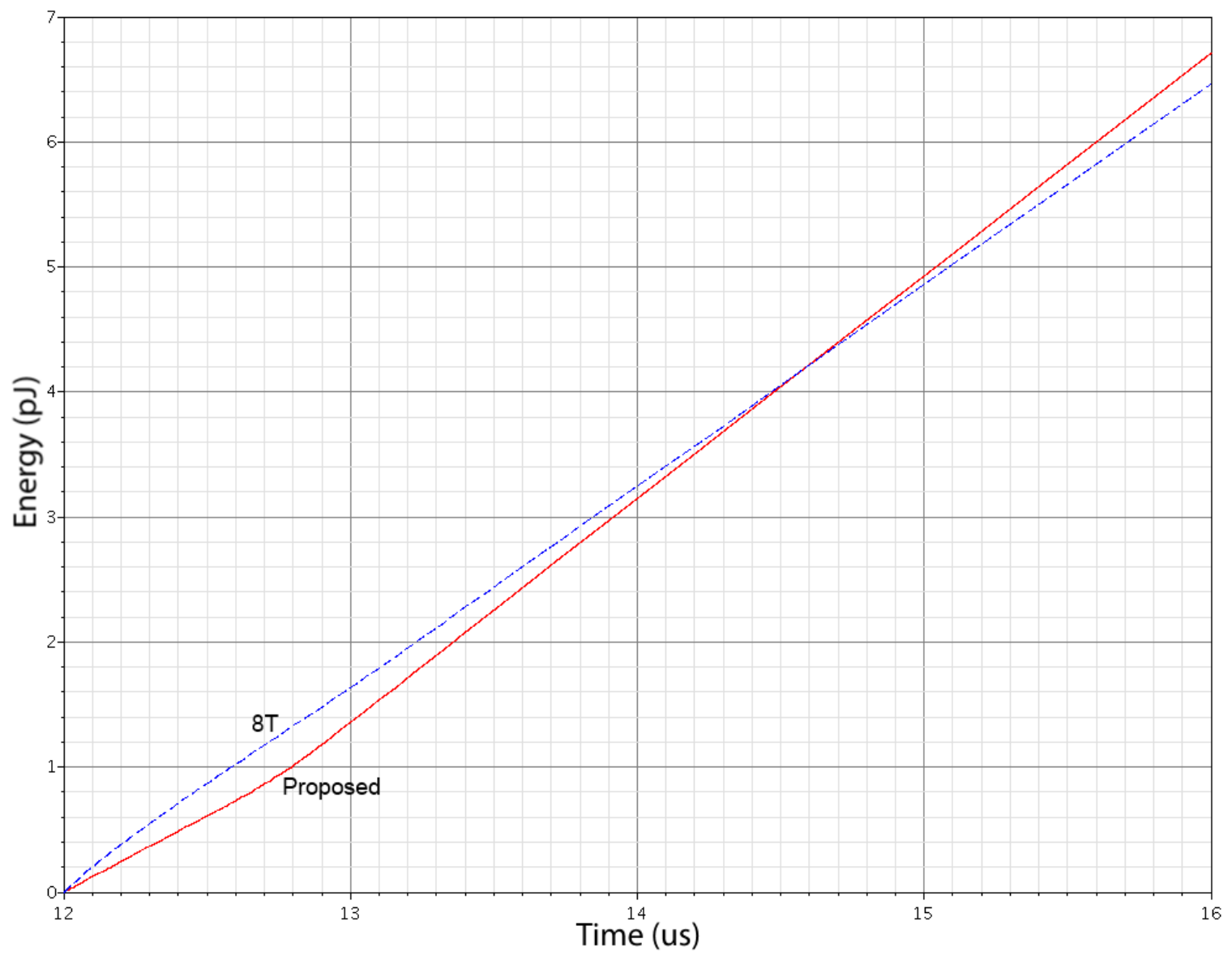

Figure 6.12: More accurate comparison of the energy consumption during a write.

power consumption once the decoder has enabled the WWL and WLA/WLB signals. Once the write operation has completed writing the new data, the power consumption again reaches a steady state. The steady state power consumption is slightly, but noticeably, higher than in the original. The increase in power consumption is because the opposite word-line (i.e. WLB instead of WLA) is enabled to write the opposite value. The value of the local bit-line in unselected blocks in the same column thus differs, and is the opposite of what it was in the first write. In this write operation, the values of the local bit-lines in unselected blocks are mostly ' 1 ', which results in higher read bit-line leakage.

In comparison to the original test, the average power consumption of the $8 \mathrm{~T}$ block during the write operation is $1.617 \mu \mathrm{W}$, and $1.677 \mu \mathrm{W}$ for the proposed block. The total energy consumption during the $4.0 \mu \mathrm{s}$ period is $6.47 \mathrm{pJ}$ for the $8 \mathrm{~T}$ block and 
$6.71 \mathrm{pJ}$ for the proposed block. If the power consumption during the actual write operation is only considered (from $12 \mu \mathrm{s}$ to $13 \mu \mathrm{s}$ ), the average power consumption of the $8 \mathrm{~T}$ block is $1.635 \mu \mathrm{W}$, or a total energy consumption of $1.64 \mathrm{pJ}$, and $1.59 \mu \mathrm{W}$ for the proposed block, or a total energy consumption of $1.36 \mathrm{pJ}$. Overall, the write operations are very comparable with a write energy consumption of $0.205 \mathrm{pJ} / \mathrm{bit}$ for the $8 \mathrm{~T}$ block and $0.17 \mathrm{pJ} / \mathrm{bit}$ for the proposed block. If the write period only consisted of the actual write operation, the proposed design definitely seems superior in terms of power consumption.

\subsubsection{Read Operation}

The power consumption during the read operation is shown in Figure 6.13. During the read operation an entire byte is read out by the block. Looking at the power consumption of the $8 \mathrm{~T}$ block, there is an immediate drop in the power consumption as the read operation begins. This drop is due to the pull-ups being disabled, leaving the bit-lines floating. When the RWL signal is enabled by the decoder, the power consumption shoots up as all the read access transistors in the selected row are enabled, selectively draining the read bit-lines. As the bit-lines slowly discharge, the power consumption drops due the decrease in leakage. A steady state is not achieved since the bit-lines are still draining at the end of the cycle. The average power consumption throughout this $4 \mu \mathrm{s}$ period is $1.947 \mu \mathrm{W}$, for a total energy consumption of $7.788 \mathrm{pJ}$ (as found in Figure 6.14).

In the proposed block, the power consumption raises once the RWL and WLB signals are raised, enabling the read access transistors for all blocks in the selected row, and access transistors for all cells in the selected column. When the global read bit-lines begin to discharge, the power begins to slowly drop. This power decrease is not as drastic as in the 8T SRAM block because 384 bit-lines (128 being read bit-lines) are discharging in the $8 \mathrm{~T}$ block, while only 16 are discharging in the proposed. This results in an average power consumption of $1.579 \mu \mathrm{W}$, and a total energy consumption of $6.317 \mathrm{pJ}$.

If only the power consumption of the actual read operation is looked at (the period between $12 \mu \mathrm{s}$ and $15 \mu \mathrm{s}$ ), the average power consumption of the $8 \mathrm{~T}$ block is $1.961 \mu \mathrm{W}$, and the average for the proposed design is $1.563 \mu \mathrm{W}$. The total energy consumption 


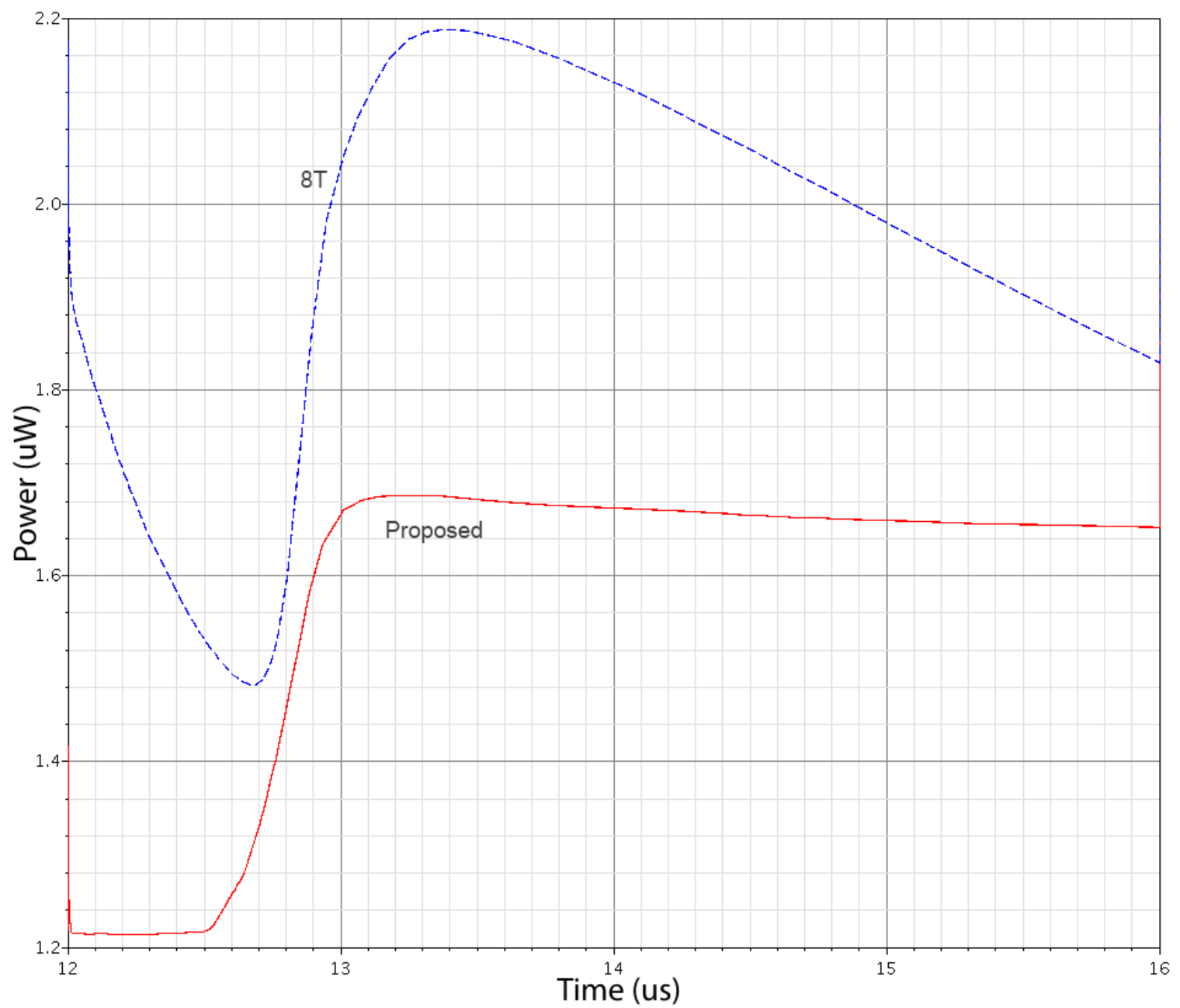

Figure 6.13: Comparison of the power consumption during a read. Close up view of Figure 6.8.

during this period is $5.884 \mathrm{pJ}$ for the $8 \mathrm{~T}$ block, and $4.688 \mathrm{pJ}$ for the proposed block. This translates to a read energy consumption of $0.7355 \mathrm{pJ} /$ bit for the $8 \mathrm{~T}$ block and $0.586 \mathrm{pJ} /$ bit for the proposed block.

\subsubsection{Idle State}

The power consumption during the idle state is relatively constant since no signals are enabled and no bit-cell is changing states. In fact, this is the static power consumption of the circuit. There is a significant decrease in power consumption at the beginning of Figure 6.15. This initial decrease is due to the decoder being slow to process the 


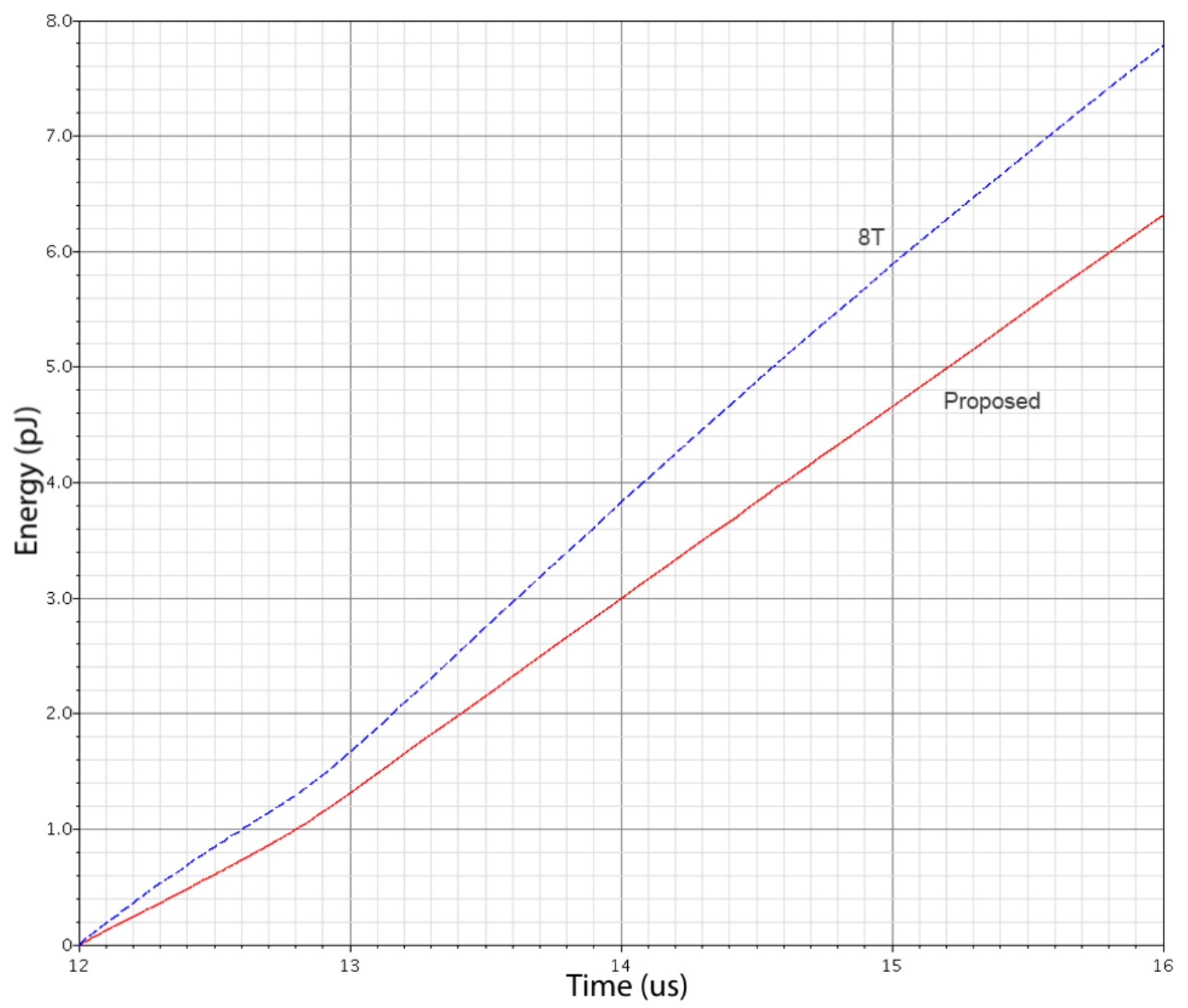

Figure 6.14: Comparison of the energy consumed during a read.

low clock state. Therefore, the idle (static) power consumption is more accurately portrayed by the period between $9-12 \mu \mathrm{s}$. In the this period, there is a slight increase in power consumption in the proposed block due to the local bit-lines reaching a steady-state voltage as a result of leakage in the bit-line.

Taking into account the entire period of the cycle, the average power consumption is $2.053 \mu \mathrm{W}$ for the $8 \mathrm{~T}$ block, and $1.251 \mu \mathrm{W}$ for the proposed block. This results in a total energy consumption of $8.21 \mathrm{pJ}$ and $5.01 \mathrm{pJ}$ for the $8 \mathrm{~T}$ and proposed blocks, respectively. If the average power consumption is measured from the period between $9-12 \mu \mathrm{s}$, the static power consumption is $1.982 \mu \mathrm{W}$ for the $8 \mathrm{~T}$ block, and $1.211 \mu \mathrm{W}$ 


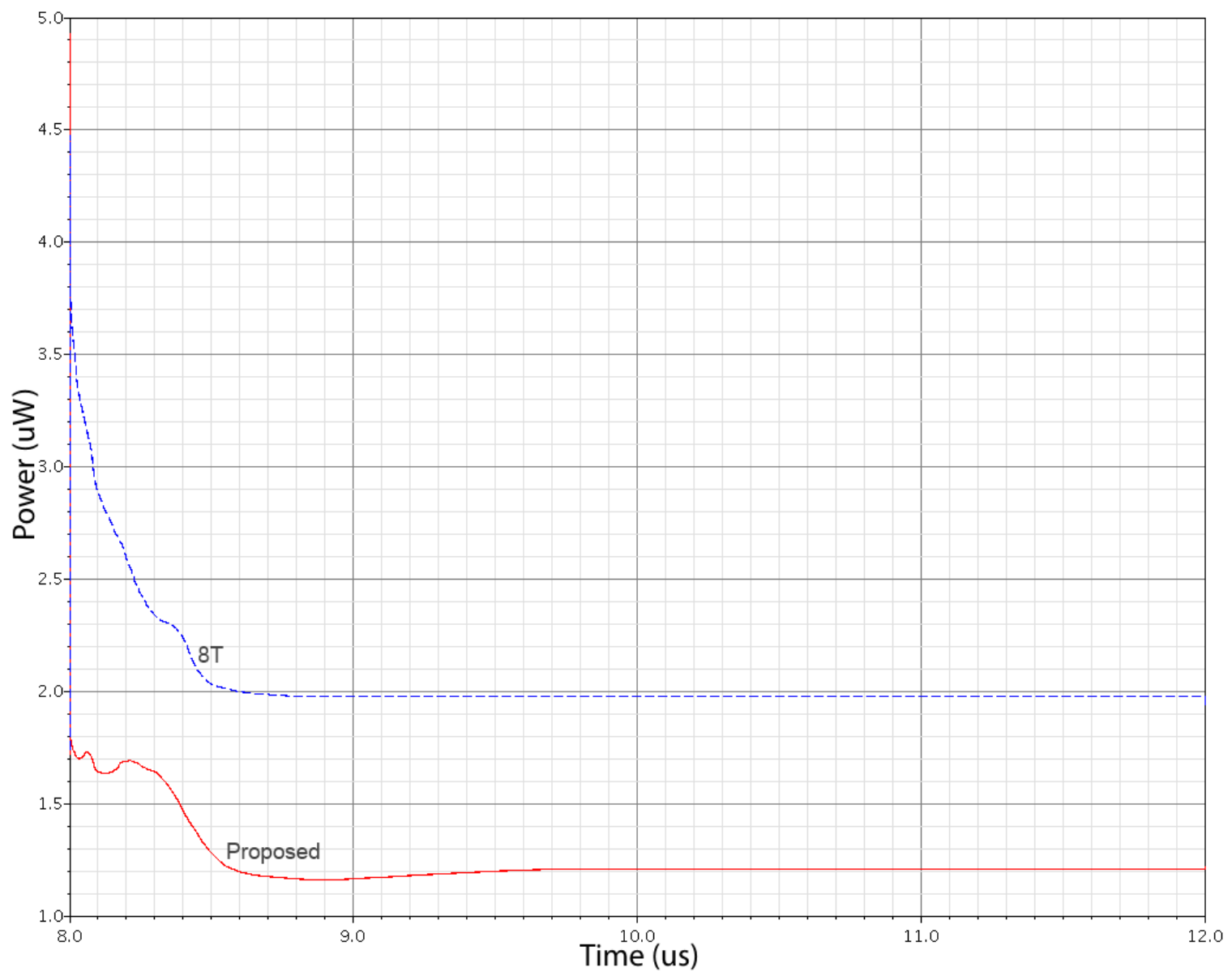

Figure 6.15: Comparison of the power consumption during the idle state, i.e. static power consumption. Close up view of Figure 6.8.

for the proposed block. In this period, the total static energy consumption is $5.934 \mathrm{pJ}$ for the $8 \mathrm{~T}$ block, and $3.616 \mathrm{pJ}$ for the proposed block.

The reason for the large discrepancy in static power consumption between the blocks is due to the $8 \mathrm{~T}$ SRAM block having 384 bit-lines that have to be pre-charged and kept high while in the idle state. The proposed block only has 16 bit-lines that need to be pre-charged and kept high. This results in significant power savings for the proposed SRAM block. 


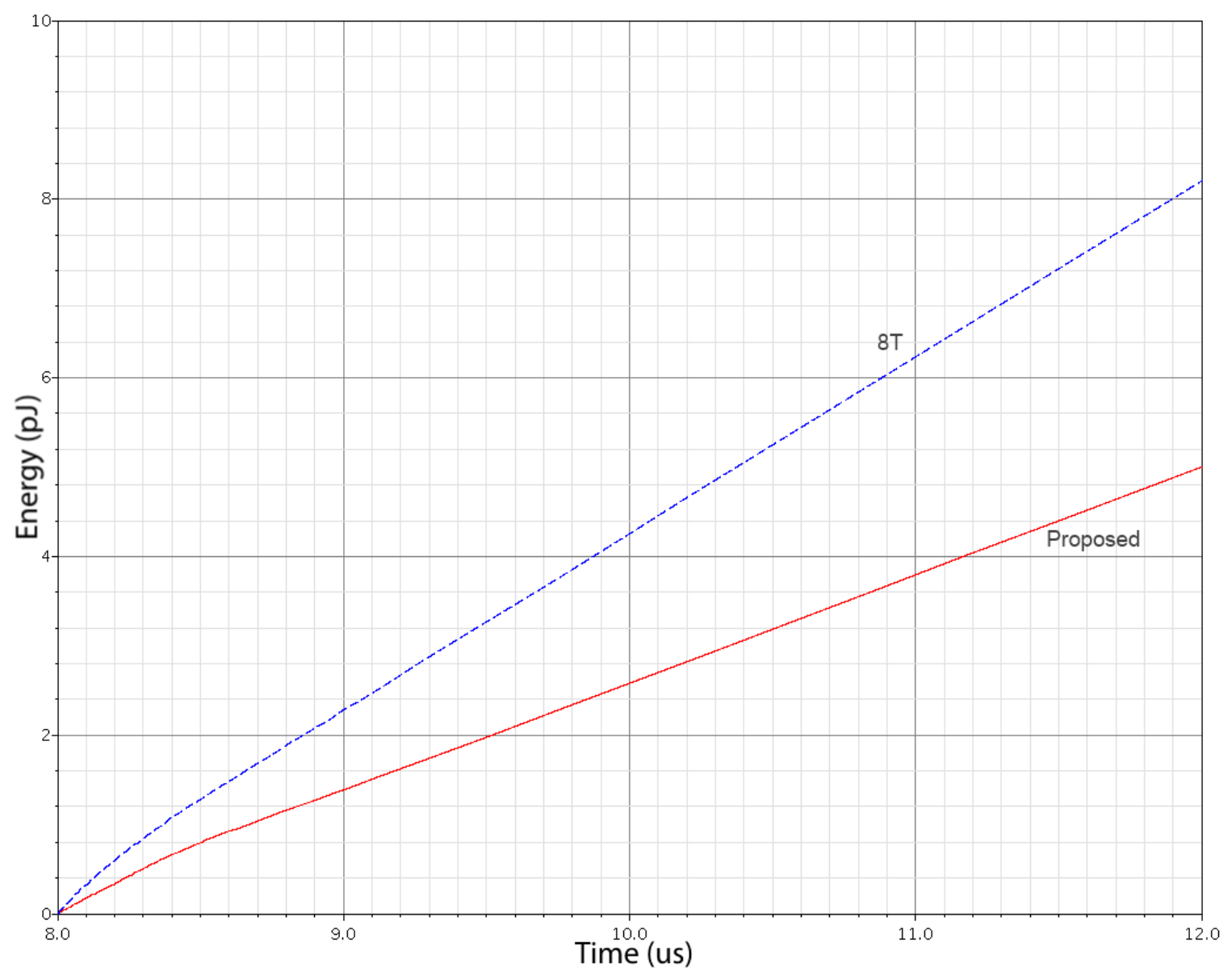

Figure 6.16: Comparison of the energy consumed during the idle state.

\subsubsection{Frequency of Operation}

By measuring the delay of the read and write operations in Figure 6.3, Figure 6.5, Figure 6.6and Figure 6.7, the maximum frequency for each operation can be calculated. The frequency of operation for the 8T SRAM block are $406.2 \mathrm{kHz}$ and $975.8 \mathrm{kHz}$ for the read and write operations respectively. This includes the decoder delay. Since the decoder is far from optimized for speed, the operation speed was also measured after the decoder was finished processing the high clock signal. This occurs when RWL and WWL for the selected row are enabled. Thus, the frequency of operation, not including the decoder delay, for the $8 \mathrm{~T}$ SRAM block are $548.8 \mathrm{kHz}$ and 2.887 $\mathrm{MHz}$ for the read and write operations respectively. Conversely, in the proposed design, the frequency of operation for the read and write operations are $399.8 \mathrm{kHz}$ and 
Table 6.2: Summary of comparison between the Proposed SRAM block and the 8T SRAM block.

\begin{tabular}{|l||c|c|c|}
\hline$V_{D D}=300 \mathrm{mV}$ & Proposed & $8 \mathrm{~T}$ & \% Improvement \\
\hline \hline Max Write speed & $\mathbf{6 . 7 0} \mathbf{~ M h z}$ & $2.887 \mathrm{MHz}$ & $132 \%$ \\
Write Power Consumption & $\mathbf{1 . 5 9} \mathbf{~ W}$ & $1.635 \mu \mathrm{W}$ & $2.75 \%$ \\
Write Energy/bit & $\mathbf{0 . 1 7} \mathbf{~ p J} / \mathbf{b i t}$ & $0.205 \mathrm{pJ} / \mathrm{bit}$ & $17.1 \%$ \\
Max Read Speed & $\mathbf{5 4 4 . 8} \mathbf{~ k H z}$ & $548.8 \mathrm{kHz}$ & $-0.73 \%$ \\
Read Power Consumption & $\mathbf{1 . 5 6 3} \mathbf{~ W}$ & $1.961 \mu \mathrm{W}$ & $20.3 \%$ \\
Read Energy/bit & $\mathbf{0 . 5 8 6} \mathbf{~ p J} / \mathbf{b i t}$ & $0.736 \mathrm{pJ} / \mathrm{bit}$ & $20.4 \%$ \\
Idle Power Consumption & $\mathbf{1 . 2 1 1} \mathbf{~ W}$ & $1.982 \mu \mathrm{W}$ & $38.9 \%$ \\
Area increase over 6T & $\mathbf{1 0 \%}$ & $\sim 30 \%$ & $17.5 \%$ \\
\hline
\end{tabular}

$1.304 \mathrm{MHz}$, respectively. When not including the decoder delay, the frequencies of operation are $544.8 \mathrm{kHz}$ and $6.70 \mathrm{Mhz}$ for the respective operations.

The results of this section is summarized in Table 6.2. The table shows that the proposed SRAM block has similar or better performance, lower operational and idle (static) power consumption, and higher integration density than 8T SRAM block.

\subsection{Comparison at Various Supply Voltages}

Since this design is intended to be a generic design, the power consumption, energy consumption per operation, and frequency of operation were measured at various supply voltages to better test and analyze it. The minimum operation voltage for the read operation was determined to be $300 \mathrm{mV}$ through testing. Below $300 \mathrm{mV}$ the worst-case leakage overwhelms the ON current of the read-decoupling transistors. When the number of rows in the array of blocks is decreased, a lower read operation voltage is obtainable. This smaller SRAM block was not thoroughly tested, however.

With this minimum operating voltage in mind, the supply voltages explored range from $300 \mathrm{mV}-500 \mathrm{mV}$. The average power consumption and total energy for each operation extends for the entire $4 \mu$ s period, not just until the operation is successfully completed. 


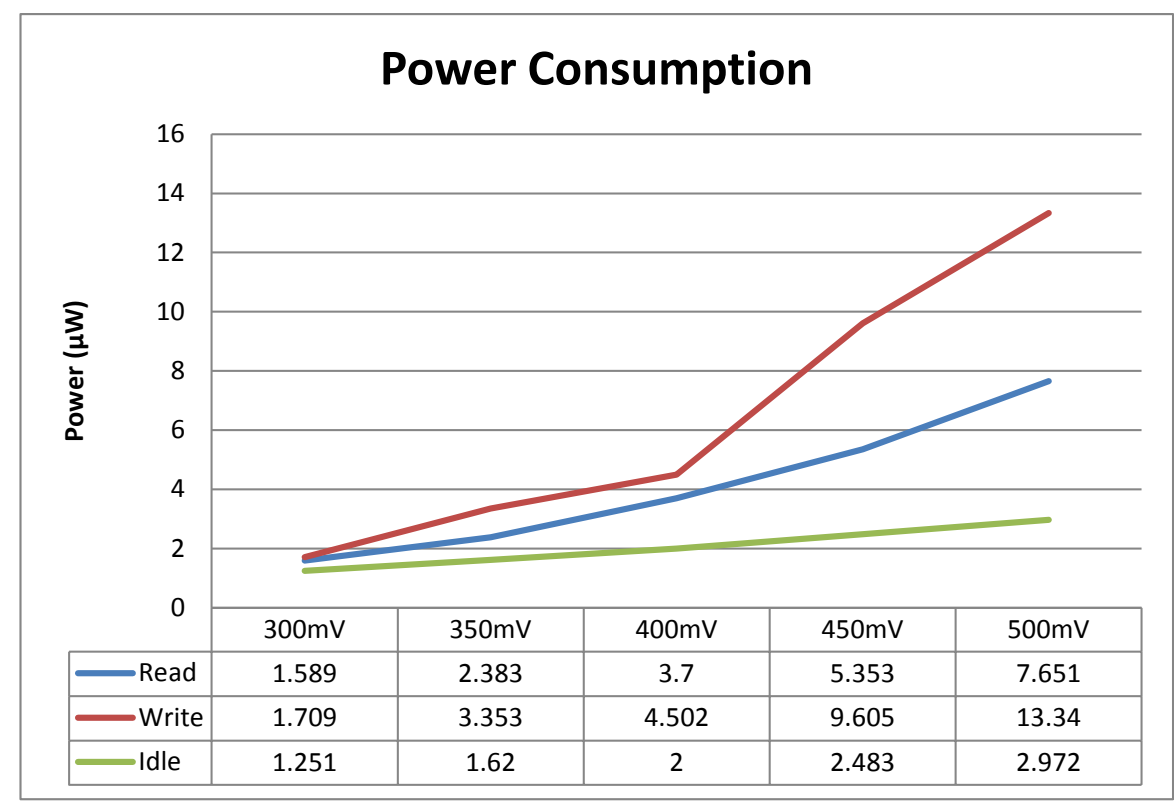

Figure 6.17: Comparison of the power consumption during the write, read, and idle operations. Simulations performed for FS corner at $27^{\circ} \mathrm{C}$.

Figure 6.17 and Figure 6.18 show that the power consumption of the write operation dramatically increases above a $V_{D D}$ of $400 \mathrm{mV}$ and that the minimum power consumption is achieved at $300 \mathrm{mV}$. It also shows that the power consumption of the read operation increases cubically as $V_{D D}$ increases. The power consumption in the idle state increases linearly as the supply voltages increases. It is, thus, advantageous to run the SRAM block at a $V_{D D}$ of $300 \mathrm{mV}$.

\subsubsection{Frequency of Operation}

Figure 6.19 and Figure 6.20 show that both the read and write speeds increase exponentially as the supply voltage increases.

With these results, it is clear that the proposed design is suitable for sub-threshold and near-threshold operation. When compared to the 8T SRAM block, it matches or beats its operation speed and power consumption at a lower transistor count. The bit array in the 8T block contains 262144 transistors, while there is only 217088 in the proposed design. This count doesn't include any of the ancillary circuitry, 


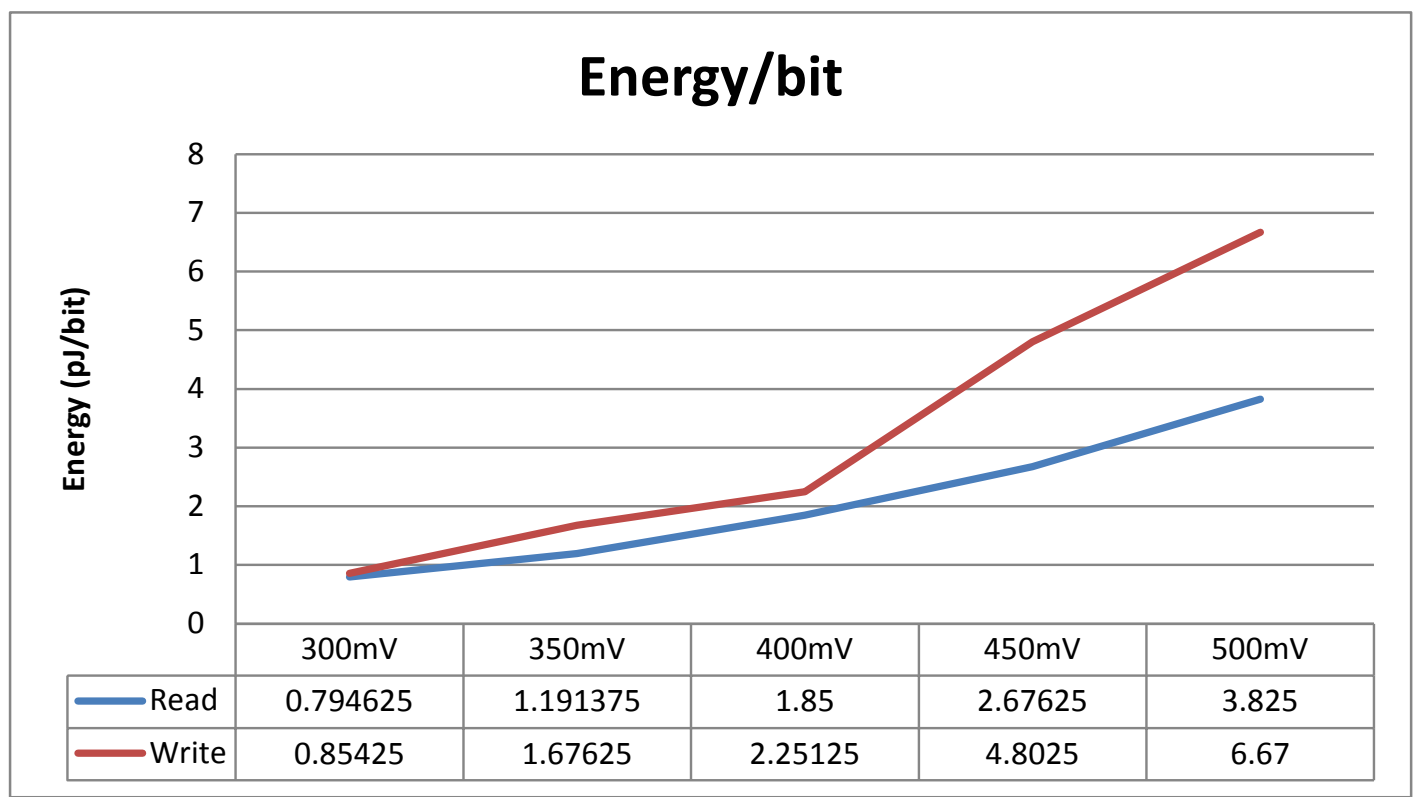

Figure 6.18: Comparison of the energy per bit consumed during the write and read operations. Simulations performed for FS corner at $27^{\circ} \mathrm{C}$.

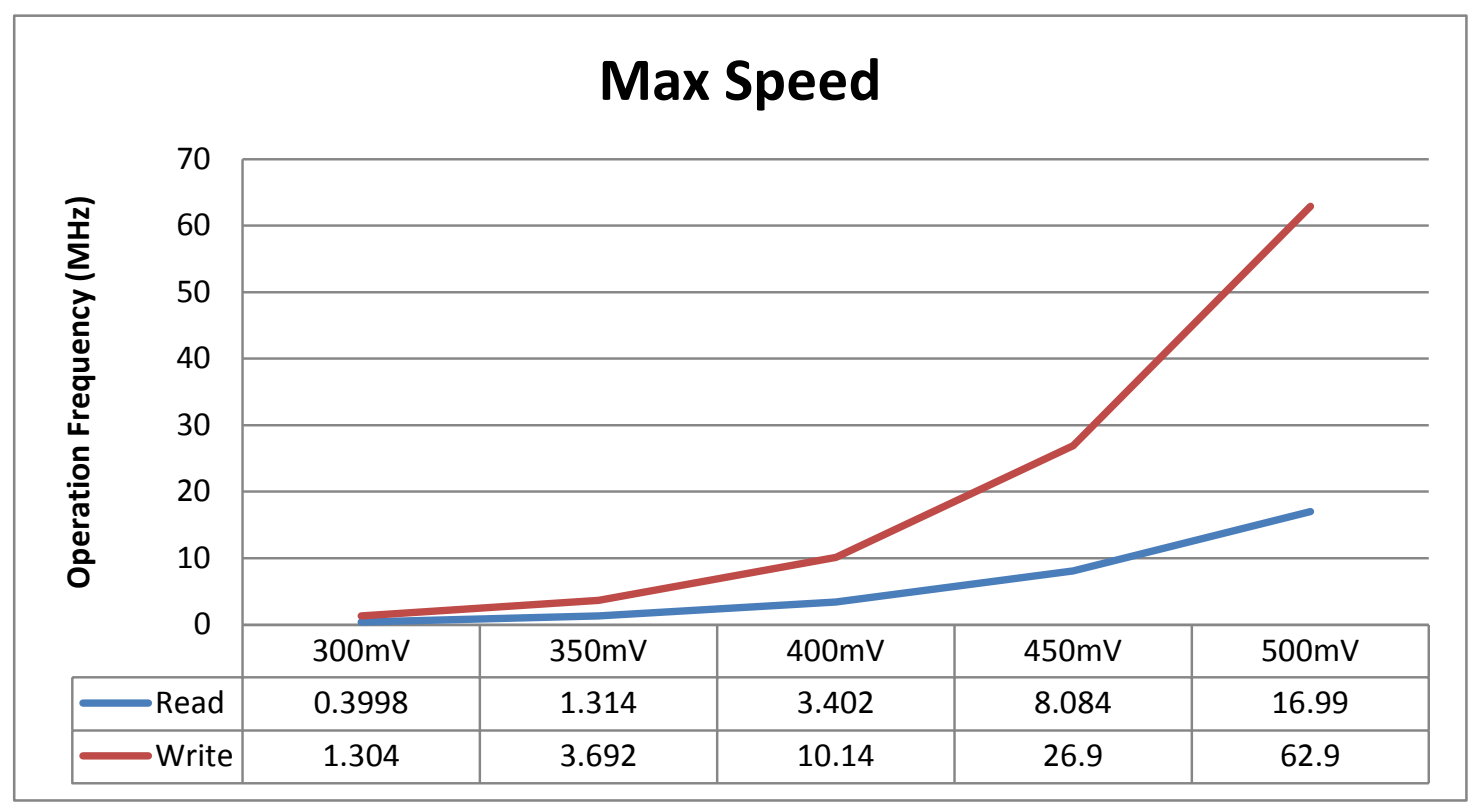

Figure 6.19: Comparison of the maximum write and read speed. Simulations performed for FS corner at $27^{\circ} \mathrm{C}$. 


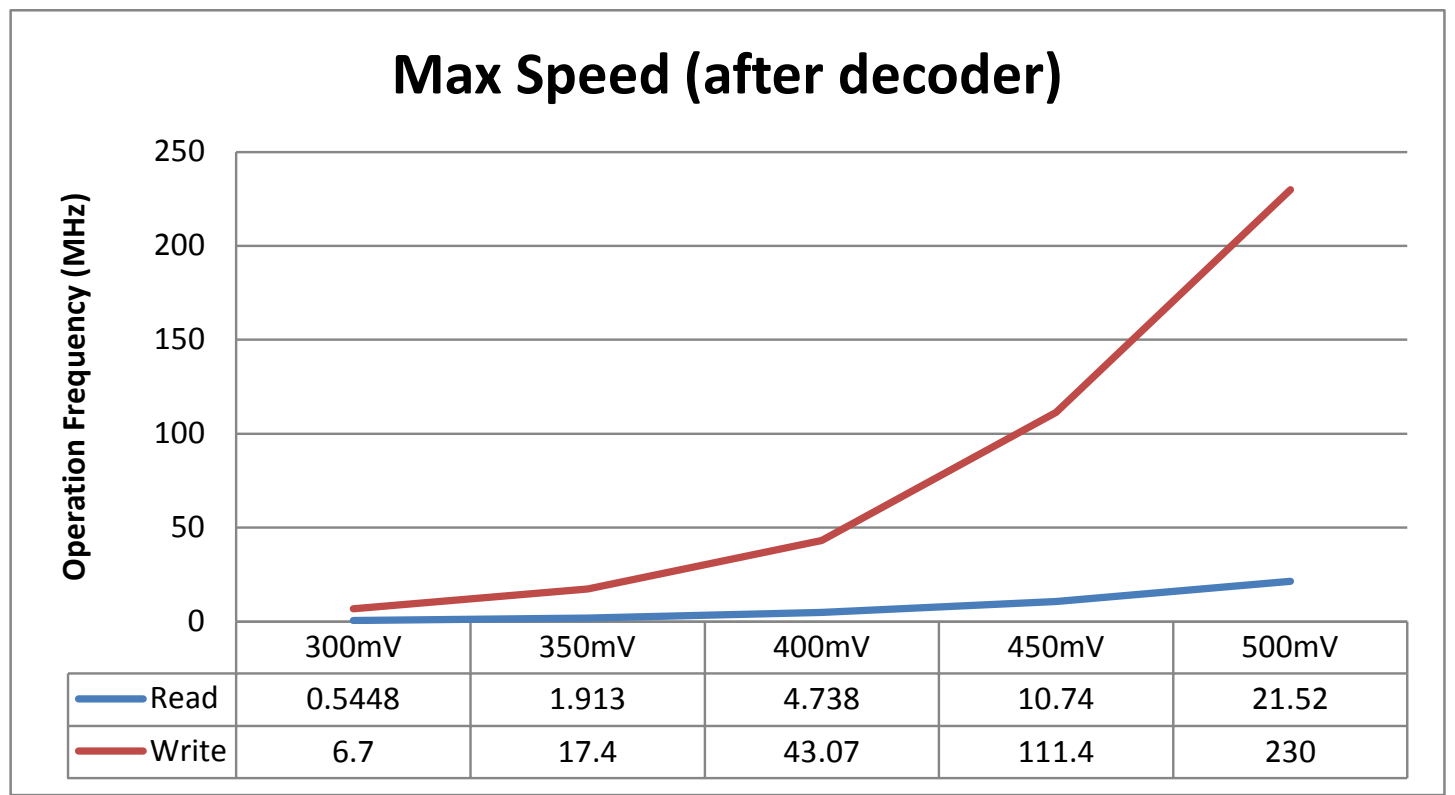

Figure 6.20: Comparison of the maximum write and read speed not including the decoder delay. Simulations performed for FS corner at $27^{\circ} \mathrm{C}$.

but the total transistor count is pretty similar in both cases. The proposed design's performance, low power consumption, and low area requirements make it an ideal substitute for the 8T SRAM in many applications. 


\section{Chapter 7}

\section{Conclusion}

This study presented a new sub- and near-threshold SRAM architecture. The proposed design's stability was thoroughly tested at the schematic level in the presence of process, temperature, and voltage variations and compared to the standard $6 \mathrm{~T}$ and traditional $8 \mathrm{~T}$ cells. Then, a 32kb SRAM block implementing the proposed architecture was designed, simulated at the schematic level, and compared to a traditional 8T SRAM cell block-showing favourable results.

Thus, this study successfully designed and implemented a new SRAM cell design that is comparable in performance to the 8T SRAM cell, but has lower operational and idle power consumption, in addition to higher integration density. The proposed SRAM architecture does all this while also mitigating half-select disturb, unlike the 8T SRAM cell, and supporting bit-interleaving. The SRAM cell is not as stable as the 8T SRAM cell, nor does its operating supply voltage go as low. However, considering that its average area per bit is very close to the standard 6T SRAM cell while still working at sub-threshold voltages, these are reasonable trade offs. Perhaps this provides a solution to the problem of a low-cost SRAM design for sub-threshold operation.

The ultra-low power embedded market is a relative niche currently. In the current market, there is perhaps a higher demand for SRAM cells that work at lower voltages, or cells that have better performance, or, in particularly the biomedical segment, cells that have increased stability, than there is for an SRAM cell that minimizes area. However, the demand is growing. With little improvement in battery technology over the past decade but rapid advancements in CMOS technologies, ultra-low power

systems may soon become more common. If that is the case, there will definitely be demand for low-cost, low-area SRAM blocks. 


\subsection{Future Work}

A number of possible areas can be tackled as an addendum to this work. It would be advantageous to improve the read SNM and reduce bit-line leakage in order to decrease the minimum read operation voltage. Numerous methods can be explored such as increasing the word-line voltage during reads, or a different bit cell structure. Perhaps the best path would be exploring separate write and read paths while retaining the horizontal bit-lines for reads may yield positive results. Alternatively, reducing the number of transistors in the read path will be advantageous to further reducing area. Using HVT transistors in only the read path may help reduce the overall transistor count, but it's debatable if this will yield lower area consumption.

It would also be interesting to explore implementing horizontal bit-lines into other SRAM architectures to see if it is universally advantageous or if this design is just an enigma. Horizontal bit-lines is certainly a fascinating idea for sub-threshold write operation. While the current proposed architecture doesn't improve write stability, it does improve power consumption and performance.

There is still a decent amount work that can be done on the proposed architecture. A vertex of lowest power consumption was not found, if there is indeed one. If possible, making the block operate at lower voltages and finding the power supply voltage that facilitates the lowest power consumption would be interesting to find. Different size SRAM blocks should be examined to see how the number of rows and columns affects the power consumption, speed, and voltage operation for the proposed design. A chip could be manufactured to properly assess the design. Even just simulating the entire layout with parasitic capacitances and resistances would be advantages to examine. Seeing how well it scales to smaller processes would be an interesting experiment as well. 


\section{List of References}

[1] Intel Newsroom, "40th anniversary of the microprocessor," 2011, Sept. [Online]. Available: http://newsroom. intel.com/docs/DOC-2383 [Accessed: Jan. 15, 2015].

[2] N. Weste and D. Harris, CMOS VLSI Design: A Circuits and Systems Perspective. USA: Addison-Wesley Publishing Company, 4th ed., 2010.

[3] E. Seevinck, F. J. List, and J. Lohstroh, "Static-noise margin analysis of mos sram cells," Solid-State Circuits, IEEE Journal of, vol. 22, no. 5, pp. 748-754, 1987.

[4] S. V. Avadhanam, "Design of a flexible high temperature sram with reduced design time," Master's thesis, Oklahoma State University, 2007.

[5] S. Nalam and B. Calhoun, "Asymmetric sizing in a 45nm 5t sram to improve read stability over 6t," in Custom Integrated Circuits Conference, 2009. CICC '09. IEEE, pp. 709-712, Sept 2009.

[6] M.-H. Tu, J.-Y. Lin, M.-C. Tsai, S.-J. Jou, and C.-T. Chuang, "Single-ended subthreshold sram with asymmetrical write/read-assist," Circuits and Systems I: Regular Papers, IEEE Transactions on, vol. 57, no. 12, pp. 3039-3047, 2010.

[7] K. Honda, K. Miyaji, S. Tanakamaru, S. Miyano, and K. Takeuchi, "Elimination of half select disturb in 8t-sram by local injected electron asymmetric pass gate transistor," in Custom Integrated Circuits Conference (CICC), 2010 IEEE, pp. 1-4, 2010.

[8] D. Anh-Tuan, J. Y. S. Low, J. Y. L. Low, Z.-H. Kong, X. Tan, and K.-S. Yeo, "An 8t differential sram with improved noise margin for bit-interleaving in $65 \mathrm{~nm}$ cmos," Circuits and Systems I: Regular Papers, IEEE Transactions on, vol. 58, no. 6, pp. 1252-1263, 2011.

[9] M. Khellah, N.-S. Kim, Y. Ye, D. Somasekhar, T. Karnik, N. Borkar, G. Pandya, F. Hamzaoglu, T. Coan, Y. Wang, K. Zhang, C. Webb, and V. De, "Process, temperature, and supply-noise tolerant $45 \mathrm{~nm}$ dense cache arrays with diffusionnotch-free (dnf) 6t sram cells and dynamic multi-vcc circuits," Solid-State Circuits, IEEE Journal of, vol. 44, no. 4, pp. 1199-1208, 2009.

[10] B. Zhai, S. Hanson, D. Blaauw, and D. Sylvester, "A variation-tolerant sub-200 mv 6-t subthreshold sram," Solid-State Circuits, IEEE Journal of, vol. 43, no. 10, pp. 2338-2348, 2008. 
[11] L.-Y. Chiou, C.-R. Huang, C.-C. Cheng, and Y.-L. Tsai, "A 300mv sub-1pj differential $6 \mathrm{t}$ sub-threshold sram with low energy and variability resilient local assist circuit," in Next-Generation Electronics (ISNE), 2013 IEEE International Symposium on, pp. 337-340, 2013.

[12] M. Khayatzadeh and Y. Lian, "Average-8t differential-sensing subthreshold sram with bit interleaving and 1k bits per bitline," 2013.

[13] M.-H. Tu, J.-Y. Lin, M.-C. Tsai, C.-Y. Lu, Y.-J. Lin, M.-H. Wang, H.-S. Huang, K.-D. Lee, W.-C. Shih, S.-J. Jou, and C.-T. Chuang, "A single-ended disturb-free $9 \mathrm{t}$ subthreshold sram with cross-point data-aware write word-line structure, negative bit-line, and adaptive read operation timing tracing," Solid-State Circuits, IEEE Journal of, vol. 47, no. 6, pp. 1469-1482, 2012.

[14] M. Nabavi and M. Shams, "A gate sizing and transistor fingering strategy for subthreshold cmos circuits," IEICE Electronics Express, vol. 9, no. 19, pp. 1550$1555,2012$.

[15] D. Markovic, C. C. Wang, L. P. Alarcon, T.-T. Liu, and J. M. Rabaey, "Ultralowpower design in near-threshold region," Proceedings of the IEEE, vol. 98, no. 2, pp. 237-252, 2010.

[16] A. Wang, B. H. Calhoun, and A. P. Chandrakasan, Sub-threshold Design for Ultra Low-Power Systems (Series on Integrated Circuits and Systems). Secaucus, NJ, USA: Springer-Verlag New York, Inc, 2006.

[17] A. Teman, A. Mordakhay, J. Mezhibovsky, and A. Fish, "A 40-nm sub-threshold 5t sram bit cell with improved read and write stability," Circuits and Systems II: Express Briefs, IEEE Transactions on, vol. 59, no. 12, pp. 873-877, 2012.

[18] K. Ishibashi and K. Osada, Low Power and Reliable SRAM Memory Cell and Array Design. Springer, 2011.

[19] A. Pavlov and M. Sachdev, CMOS SRAM Circuit Design and Parametric Test in Nano-Scaled Technologies: Process-Aware SRAM Design and Test. Springer, 2008. 2008924192.

[20] B. H. Calhoun and A. Chandrakasan, "A 256-kb 65-nm sub-threshold sram design for ultra-low-voltage operation," Solid-State Circuits, IEEE Journal of, vol. 42, no. 3, pp. 680-688, 2007.

[21] F. Moradi, G. Panagopoulos, G. Karakonstantis, D. Wisland, H. Mahmoodi, J. K. Madsen, and K. Roy, "Multi-level wordline driver for low power srams in nano-scale cmos technology," in Proceedings of the 2011 IEEE 29th International Conference on Computer Design, ICCD '11, (Washington, DC, USA), pp. 326331, IEEE Computer Society, 2011.

[22] M. E. Sinangil, N. Verma, and A. Chandrakasan, "A reconfigurable 8t ultradynamic voltage scalable (u-dvs) sram in $65 \mathrm{~nm}$ cmos," Solid-State Circuits, IEEE Journal of, vol. 44, no. 11, pp. 3163-3173, 2009. 
[23] B. Zimmer, S. O. Toh, H. Vo, Y. Lee, O. Thomas, K. Asanovic, and B. Nikolic, "Sram assist techniques for operation in a wide voltage range in 28-nm cmos," Circuits and Systems II: Express Briefs, IEEE Transactions on, vol. 59, no. 12, pp. 853-857, 2012.

[24] M. Khellah, Y. Ye, N. S. Kim, D. Somasekhar, G. Pandya, A. Farhang, K. Zhang, C. Webb, and V. De, "Wordline \& bitline pulsing schemes for improving sram cell stability in low-vcc 65nm cmos designs," in VLSI Circuits, 2006. Digest of Technical Papers. 2006 Symposium on, pp. 9-10, 2006.

[25] N. Verma and A. Chandrakasan, "A $256 \mathrm{~kb} 65 \mathrm{~nm}$ 8t subthreshold sram employing sense-amplifier redundancy," Solid-State Circuits, IEEE Journal of, vol. 43, no. 1, pp. 141-149, 2008.

[26] F. Bauer, G. Georgakos, and D. Schmitt-Landsiedel, A Design Space Comparison of 6 T and $8 T$ SRAM Core-Cells, vol. 5349 of Lecture Notes in Computer Science. Springer Berlin Heidelberg, 2009.

[27] A. K. Singh, Digital Vlsi Design. PHI Learning Pvt. Ltd, 2010.

[28] M.-H. Chang, Y.-T. Chiu, S.-L. Lai, and W. Hwang, "A 1kb 9t subthreshold sram with bit-interleaving scheme in 65nm cmos," in Low Power Electronics and Design (ISLPED) 2011 International Symposium on, pp. 291-296, 2011.

[29] M. Jagasivamani, "Development of a low-power sram compiler," Master's thesis, Virginia Polytechnic Institute and State University, 2000.

[30] J. P. Kulkarni, A. Goel, P. Ndai, and K. Roy, "A read-disturb-free, differential sensing 1r/1w port, 8t bitcell array," Very Large Scale Integration (VLSI) Systems, IEEE Transactions on, vol. 19, no. 9, pp. 1727-1730, 2011.

[31] T.-H. Kim, J. Liu, J. Keane, and C. H. Kim, "A 0.2 v, 480 kb subthreshold sram with 1 k cells per bitline for ultra-low-voltage computing," Solid-State Circuits, IEEE Journal of, vol. 43, no. 2, pp. 518-529, 2008.

[32] T.-H. Kim, J. Liu, and C. H. Kim, "A voltage scalable 0.26 v, 64 kb 8t sram with v lowering techniques and deep sleep mode," Solid-State Circuits, IEEE Journal of, vol. 44, no. 6, pp. 1785-1795, 2009.

[33] M.-E. Hwang and K. Roy, "A 135mv 0.13w process tolerant 6t subthreshold dtmos sram in 90nm technology," in Custom Integrated Circuits Conference, 2008. CICC 2008. IEEE, pp. 419-422, 2008.

[34] S. Soleimani-Amiri, A. Afzali-Kusha, and A. Sammak, "Design and analysis of a new sub-threshold dtmos sram cell structure," in Computer Architecture and Digital Systems (CADS), 2012 16th CSI International Symposium on, pp. 50-53, 2012.

[35] J. P. Kulkarni and K. Roy, "Ultralow-voltage process-variation-tolerant schmitttrigger-based sram design," Very Large Scale Integration (VLSI) Systems, IEEE Transactions on, vol. 20, no. 2, pp. 319-332, 2012. 
[36] G. Pasandi and S. M. Fakhraie, "A new sub-threshold 7t sram cell design with capability of bit-interleaving in $90 \mathrm{~nm}$ cmos," in Electrical Engineering (ICEE), 2013 21st Iranian Conference on, pp. 1-6, 2013.

[37] N. BAI, X. WU, J. YANG, and L. SHI, "A robust high density 7t sram bitcell for subthreshold applications," Chinese Journal of Electronics, vol. 20, no. 2, pp. 243-246, 2011.

[38] M.-F. Chang, S.-W. Chang, P.-W. Chou, and W.-C. Wu, "A 130 mv sram with expanded write and read margins for subthreshold applications," Solid-State Circuits, IEEE Journal of, vol. 46, no. 2, pp. 520-529, 2011.

[39] Z. Liu and V. Kursun, "Characterization of a novel nine-transistor sram cell," Very Large Scale Integration (VLSI) Systems, IEEE Transactions on, vol. 16, no. 4, pp. 488-492, 2008.

[40] R. V. Joshi, R. Kanj, and V. Ramadurai, "A novel column-decoupled 8t cell for low-power differential and domino-based sram design," Very Large Scale Integration (VLSI) Systems, IEEE Transactions on, vol. 19, no. 5, pp. 869-882, 2011.

[41] J.-J. Wu, Y.-H. Chen, M.-F. Chang, P.-W. Chou, C.-Y. Chen, H.-J. Liao, M.B. Chen, Y.-H. Chu, W.-C. Wu, and H. Yamauchi, "A large v/vdd tolerant zigzag 8t sram with area-efficient decoupled differential sensing and fast writeback scheme," Solid-State Circuits, IEEE Journal of, vol. 46, no. 4, pp. 815-827, 2011.

[42] V. Sharma, S. Cosemans, M. Ashouei, J. Huisken, F. Catthoor, and W. Dehaene, "Ultra low power litho friendly local assist circuitry for variability resilient 8t sram," in Design, Automation \&6 Test in Europe Conference \&6 Exhibition (DATE), 2012, pp. 1042-1047, 2012.

[43] I.-J. Chang, J.-J. Kim, S. P. Park, and K. Roy, "A 32 kb 10t sub-threshold sram array with bit-interleaving and differential read scheme in $90 \mathrm{~nm}$ cmos," Solid-State Circuits, IEEE Journal of, vol. 44, no. 2, pp. 650-658, 2009.

[44] M. Yabuuchi, K. Nii, Y. Tsukamoto, S. Ohbayashi, Y. Nakase, and H. Shinohara, "A 45nm 0.6v cross-point 8t sram with negative biased read/write assist," in VLSI Circuits, 2009 Symposium on, pp. 158-159, 2009.

[45] A. Bhattacharyya, Compact MOSFET Models for VLSI Design. Wiley, 2009.

[46] N. Shigyo and T. Hiraoka, "A review of narrow-channel effects for $\{$ STI $\}$ mosfet's: A difference between surface- and buried-channel cases," Solid-State Electronics, vol. 43, no. 11, pp. $2061-2066,1999$.

[47] A. Wang, B. Calhoun, and A. Chandrakasan, Sub-threshold Design for Ultra Low-Power Systems. Integrated Circuits and Systems, Springer, 2006.

[48] K. Roy, S. Mukhopadhyay, and H. Mahmoodi-Meimand, "Leakage current mechanisms and leakage reduction techniques in deep-submicrometer cmos circuits," Proceedings of the IEEE, vol. 91, pp. 305-327, Feb 2003. 
[49] T. P. Ryan, Modern Engineering Statistics. Wiley, 2007.

[50] K. Iniewski, Advanced Circuits for Emerging Technologies. Wiley, 2012.

[51] MATLAB, version 8.3.0.532 (R2014a). Natick, Massachusetts: The MathWorks Inc., 2014. 


\section{Appendix A}

\section{Static Noise Margin Simulation Results}

\section{A.1 Hold Operation}

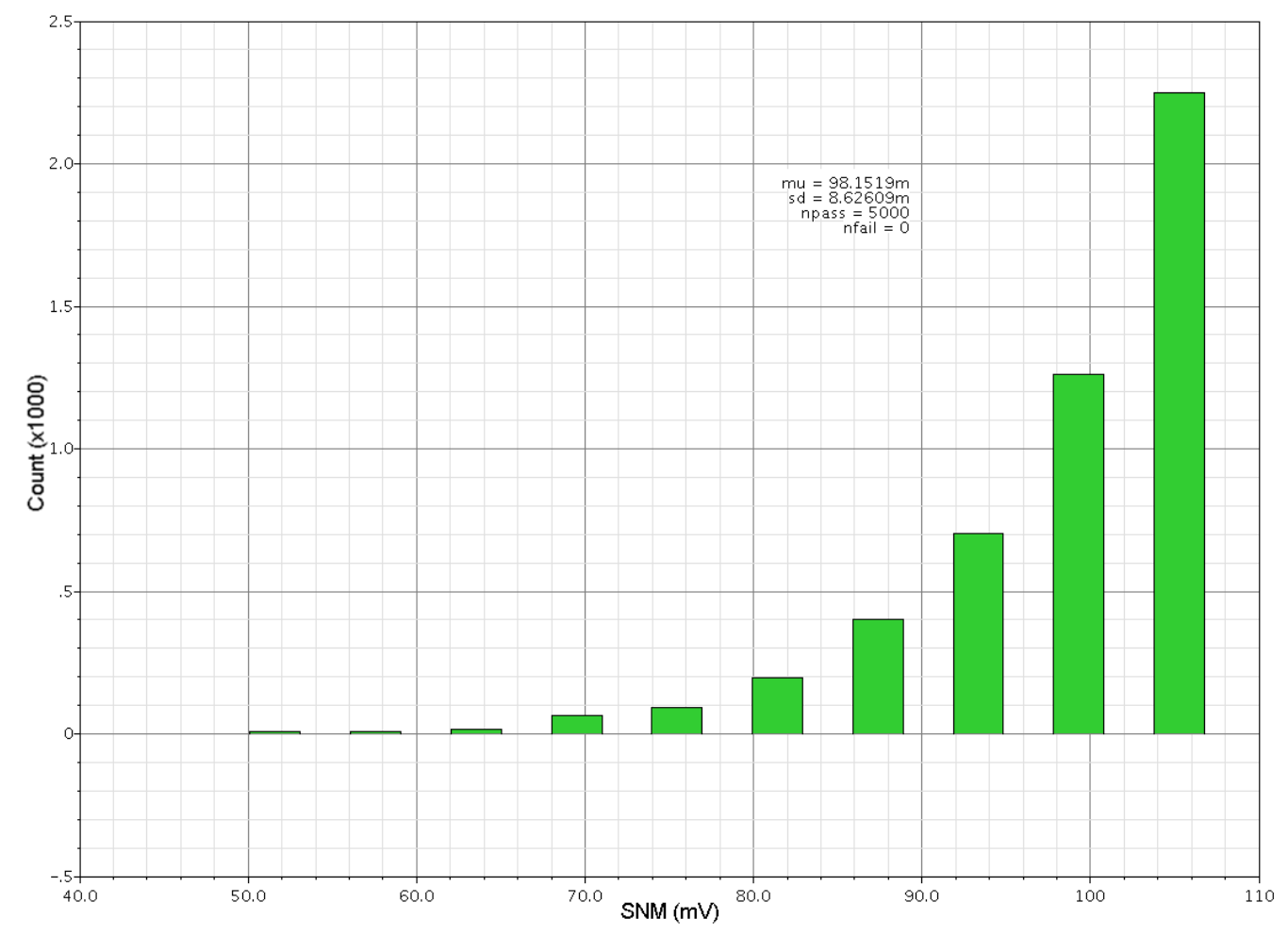

Figure A.1: Histogram for the hold operation of the 8-cell block configuration. $V_{D D}$ is $300 \mathrm{mV}$ and the temperature is $27^{\circ} \mathrm{C}$. Only the 8-cell configuration is included because all configurations have very similar distributions. This is similarly true for all sub-threshold operating voltages. 


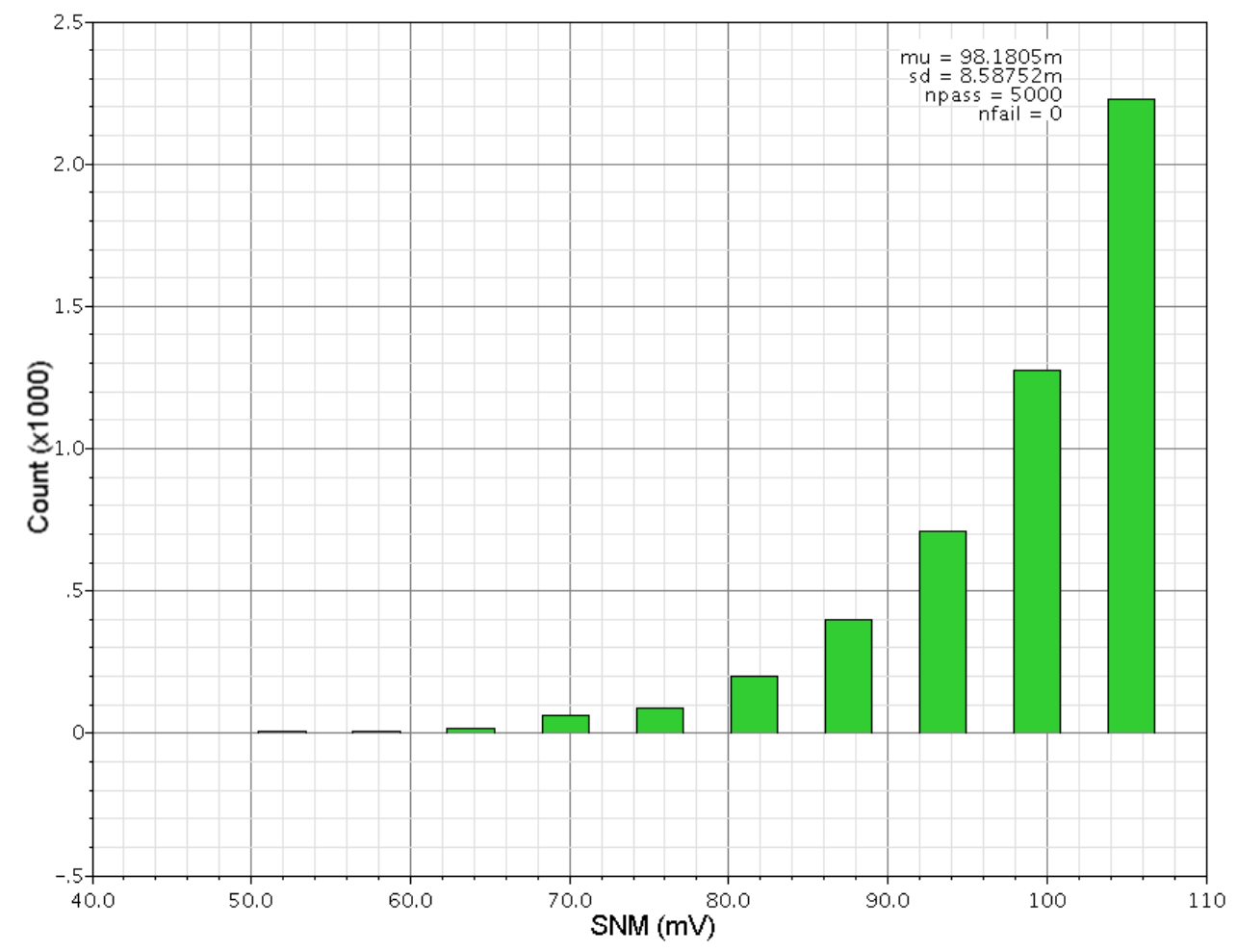

Figure A.2: Histogram for the hold operation of the traditional $8 \mathrm{~T}$ cell. $V_{D D}$ is $300 \mathrm{mV}$ and the temperature is $27^{\circ} \mathrm{C}$. 


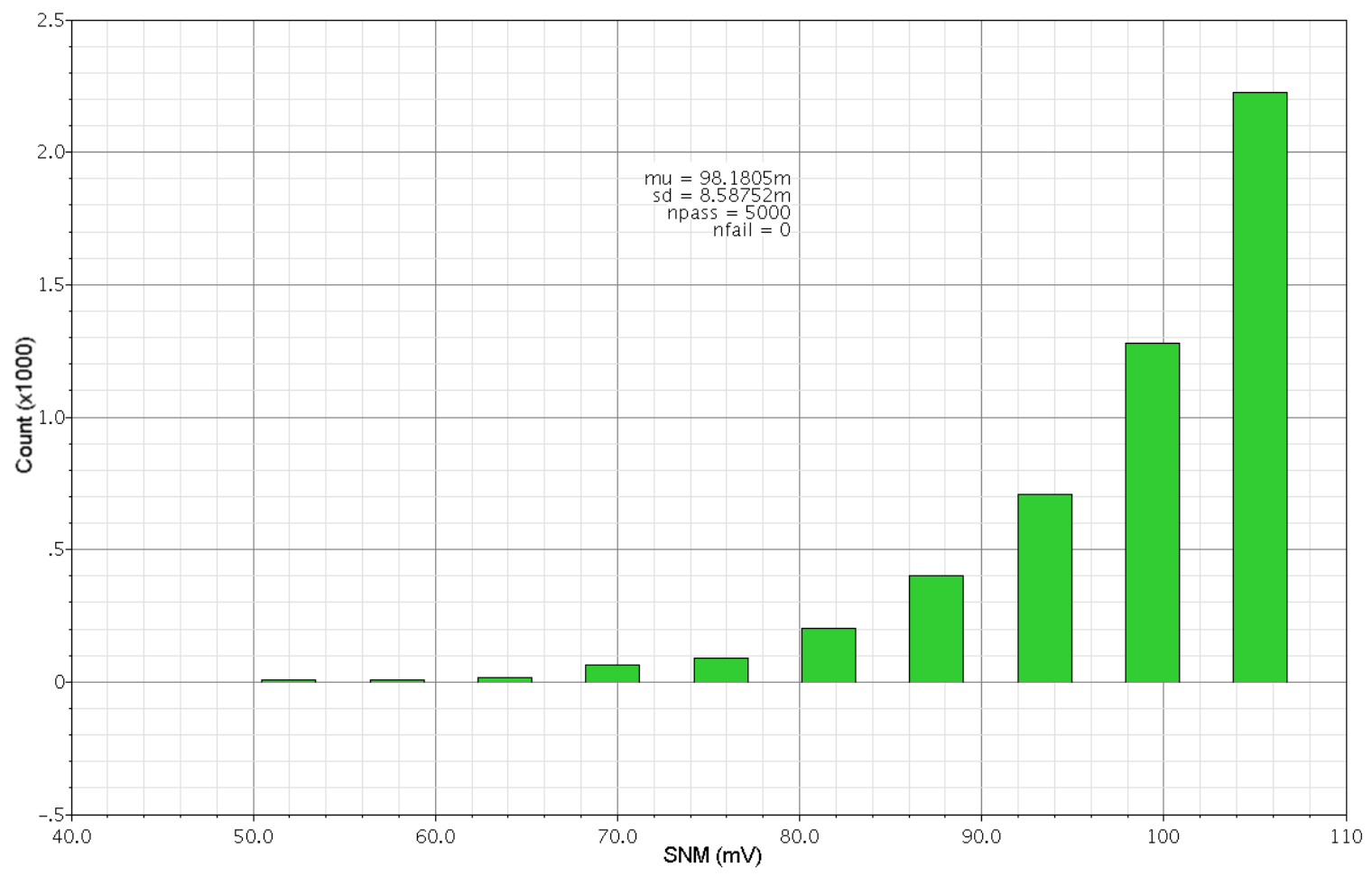

Figure A.3: Histogram for the hold operation of the standard $6 \mathrm{~T}$ cell. $V_{D D}$ is $300 \mathrm{mV}$ and the temperature is $27^{\circ} \mathrm{C}$. 
Table A.1: 1-cell Configuration

Hold SNM

\begin{tabular}{|l|c|c|c|c|}
\hline$V_{D D}$ & $\mu$ & $\sigma$ & $\mu-4 \sigma$ & $\mu-5 \sigma$ \\
\hline $200 \mathrm{mV}$ & 50.9937 & 9.16324 & 14.34074 & 5.1775 \\
$250 \mathrm{mV}$ & 74.6007 & 8.94069 & 38.83794 & 29.89725 \\
$275 \mathrm{mV}$ & 86.3046 & 8.87616 & 50.79996 & 41.9238 \\
$300 \mathrm{mV}$ & 97.9107 & 8.81488 & 62.65118 & 53.8363 \\
$350 \mathrm{mV}$ & 120.97 & 8.80096 & 85.76616 & 76.9652 \\
\hline
\end{tabular}

Table A.2: 2-cell Configuration

Hold SNM

\begin{tabular}{|l|c|c|c|c|}
\hline$V_{D D}$ & $\mu$ & $\sigma$ & $\mu-4 \sigma$ & $\mu-5 \sigma$ \\
\hline $200 \mathrm{mV}$ & 51.2282 & 9.03835 & 15.0748 & 6.03645 \\
$250 \mathrm{mV}$ & 74.7571 & 8.83082 & 39.43382 & 30.603 \\
$275 \mathrm{mV}$ & 84.947 & 9.47921 & 47.03016 & 37.55095 \\
$300 \mathrm{mV}$ & 97.9994 & 8.74372 & 63.02452 & 54.2808 \\
$350 \mathrm{mV}$ & 121.015 & 8.76163 & 85.96848 & 77.20685 \\
\hline
\end{tabular}


Table A.3: 4-cell Configuration

Hold SNM

\begin{tabular}{|l|c|c|c|c|}
\hline$V_{D D}$ & $\mu$ & $\sigma$ & $\mu-4 \sigma$ & $\mu-5 \sigma$ \\
\hline $200 \mathrm{mV}$ & 51.4155 & 8.93937 & 15.65802 & 6.71865 \\
$250 \mathrm{mV}$ & 74.8944 & 8.73782 & 39.94312 & 31.2053 \\
$275 \mathrm{mV}$ & 84.9524 & 9.47568 & 47.04968 & 37.574 \\
$300 \mathrm{mV}$ & 98.0853 & 8.67716 & 63.37666 & 54.6995 \\
$350 \mathrm{mV}$ & 121.064 & 8.72073 & 86.18108 & 77.46035 \\
\hline
\end{tabular}

Table A.4: 8-cell Configuration

Hold SNM

\begin{tabular}{|l|c|c|c|c|}
\hline$V_{D D}$ & $\mu$ & $\sigma$ & $\mu-4 \sigma$ & $\mu-5 \sigma$ \\
\hline $200 \mathrm{mV}$ & 51.5421 & 8.86813 & 16.06958 & 7.20145 \\
$250 \mathrm{mV}$ & 74.9925 & 8.67106 & 40.30826 & 31.6372 \\
$275 \mathrm{mV}$ & 86.6172 & 8.64551 & 52.03516 & 43.38965 \\
$300 \mathrm{mV}$ & 98.1519 & 8.62609 & 63.64754 & 55.02145 \\
$350 \mathrm{mV}$ & 121.105 & 8.68699 & 86.35704 & 77.67005 \\
\hline
\end{tabular}

Table A.5: Traditional 8T

Hold SNM

\begin{tabular}{|l|c|c|c|c|}
\hline$V_{D D}$ & $\mu$ & $\sigma$ & $\mu-4 \sigma$ & $\mu-5 \sigma$ \\
\hline $200 \mathrm{mV}$ & 51.8192 & 8.51484 & 17.75984 & 9.245 \\
$250 \mathrm{mV}$ & 75.085 & 8.53884 & 40.92964 & 32.3908 \\
$275 \mathrm{mV}$ & 86.6686 & 8.57489 & 52.36904 & 43.79415 \\
$300 \mathrm{mV}$ & 98.1805 & 8.58752 & 63.83042 & 55.2429 \\
$350 \mathrm{mV}$ & 121.116 & 8.67459 & 86.41764 & 77.74305 \\
\hline
\end{tabular}


Table A.6: Standard 6T

\begin{tabular}{|l|c|c|c|c|}
\multicolumn{5}{|c}{ Hold SNM } \\
\hline$V_{D D}$ & $\mu$ & $\sigma$ & $\mu-4 \sigma$ & $\mu-5 \sigma$ \\
\hline $200 \mathrm{mV}$ & 51.8192 & 8.51484 & 17.75984 & 9.245 \\
$250 \mathrm{mV}$ & 75.085 & 8.53884 & 40.92964 & 32.3908 \\
$275 \mathrm{mV}$ & 86.6456 & 8.59021 & 52.28476 & 43.69455 \\
$300 \mathrm{mV}$ & 98.1805 & 8.58752 & 63.83042 & 55.2429 \\
$350 \mathrm{mV}$ & 121.116 & 8.67459 & 86.41764 & 77.74305 \\
\hline
\end{tabular}




\section{A.2 Write Operation}

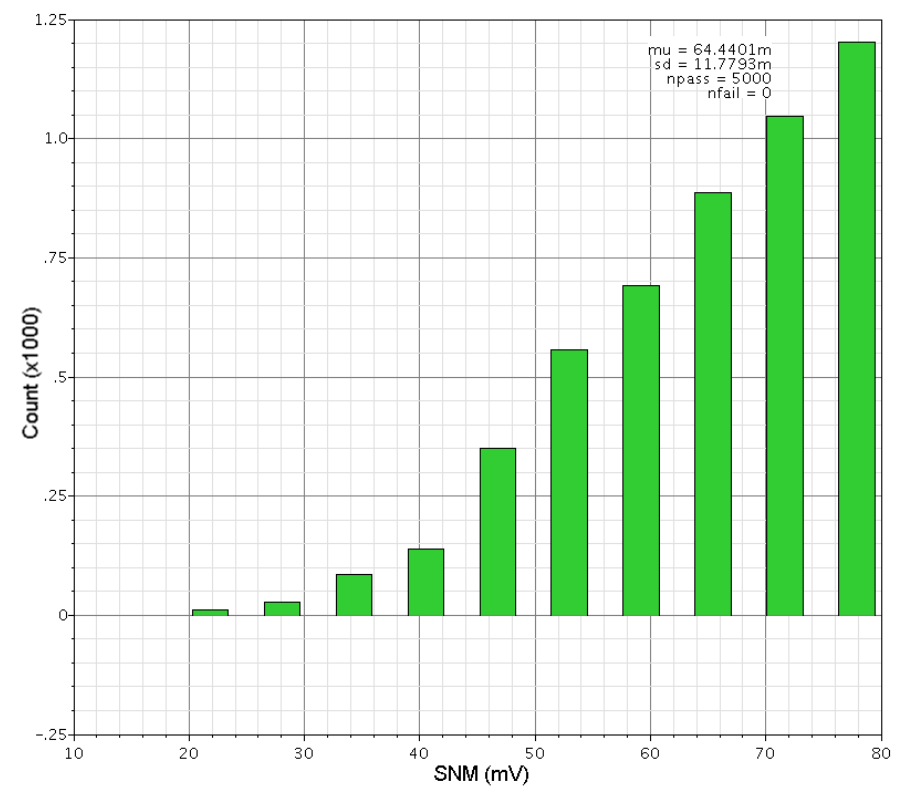

Figure A.4: Histogram for the write operation of the 8-cell block configuration. $V_{D D}$ is $200 \mathrm{mV}$ and the temperature is $27^{\circ} \mathrm{C}$.

Table A.7: 1-cell Configuration

\begin{tabular}{|l|c|c|c|c|}
\multicolumn{5}{|c}{ Write SNM } \\
\hline$V_{D D}$ & $\mu$ & $\sigma$ & $\mu-4 \sigma$ & $\mu-5 \sigma$ \\
\hline $200 \mathrm{mV}$ & 65.6669 & 11.7205 & 18.7849 & 7.0644 \\
$250 \mathrm{mV}$ & 92.4534 & 13.5829 & 38.1218 & 24.5389 \\
$275 \mathrm{mV}$ & 105.513 & 14.259 & 48.477 & 34.218 \\
$300 \mathrm{mV}$ & 118.309 & 14.8241 & 59.0126 & 44.1885 \\
$350 \mathrm{mV}$ & 143.12 & 15.6947 & 80.3412 & 64.6465 \\
\hline
\end{tabular}




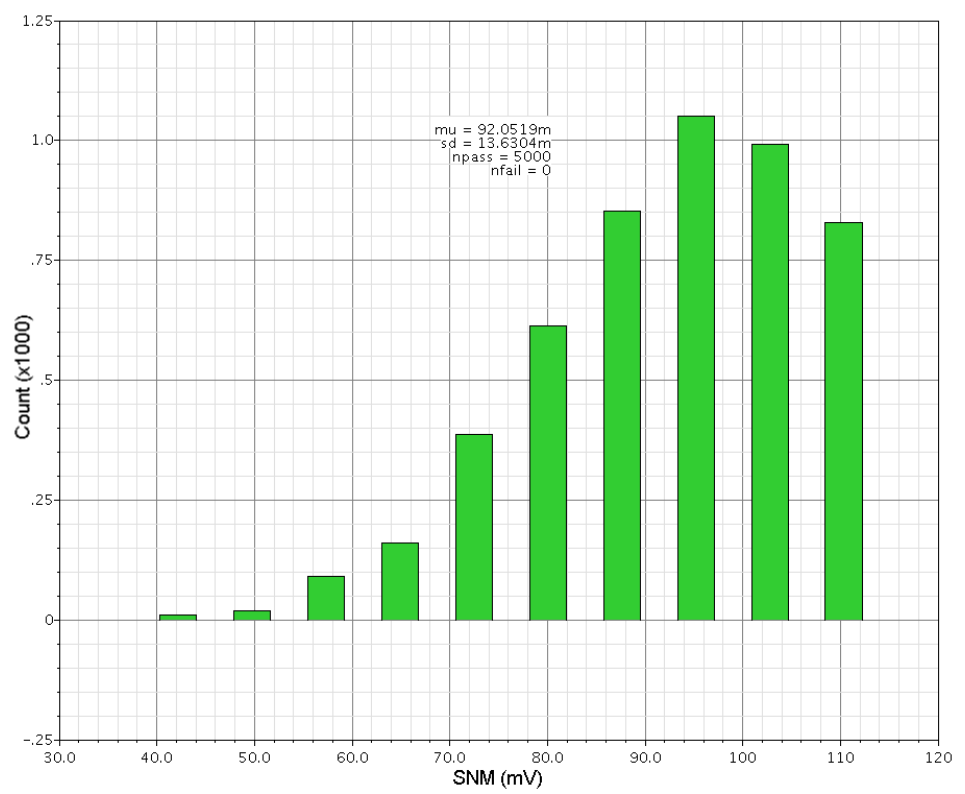

Figure A.5: Histogram for the write operation of the 8-cell block configuration. $V_{D D}$ is $250 \mathrm{mV}$ and the temperature is $27^{\circ} \mathrm{C}$.

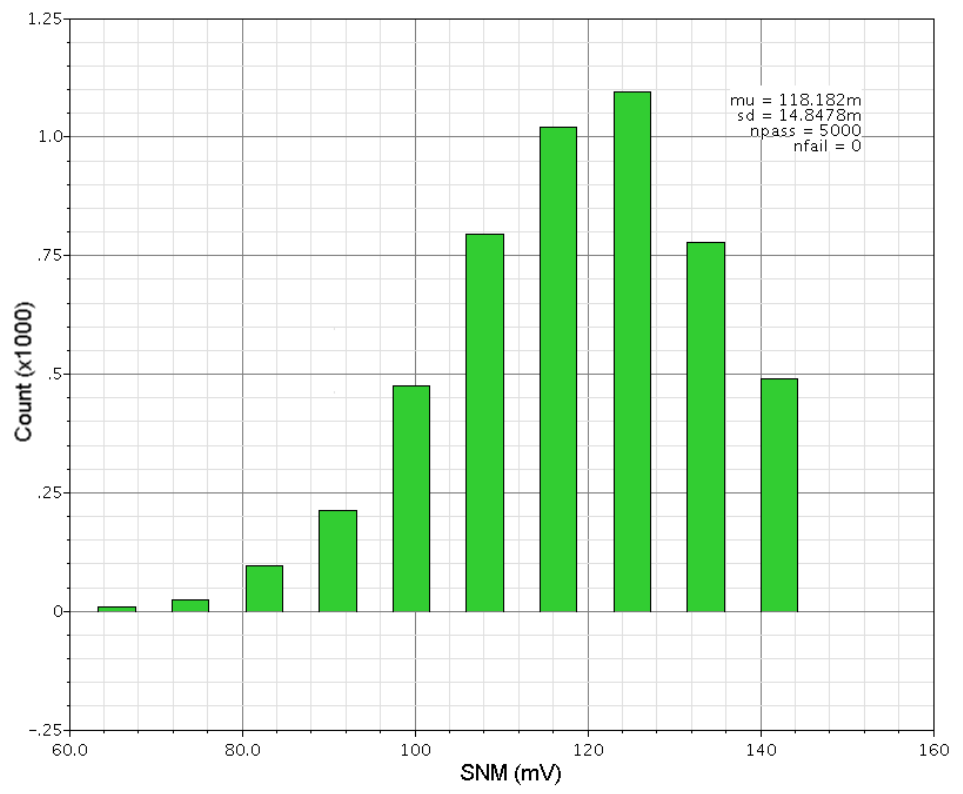

Figure A.6: Histogram for the write operation of the 8-cell block configuration. $V_{D D}$ is $300 \mathrm{mV}$ and the temperature is $27^{\circ} \mathrm{C}$. 


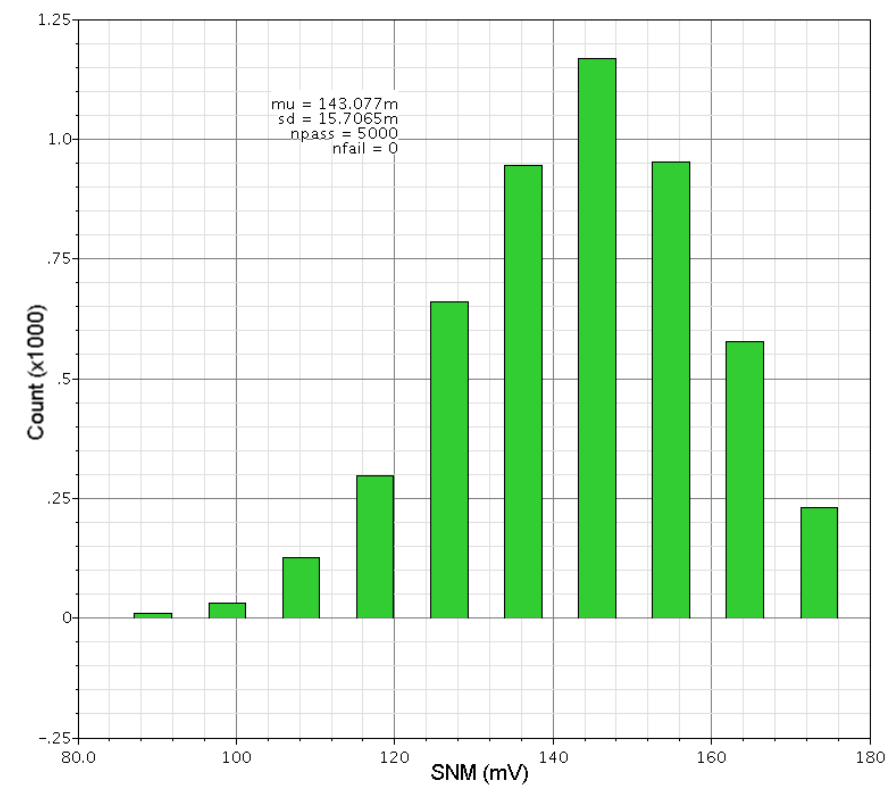

Figure A.7: Histogram for the write operation of the 8-cell block configuration. $V_{D D}$ is $350 \mathrm{mV}$ and the temperature is $27^{\circ} \mathrm{C}$.

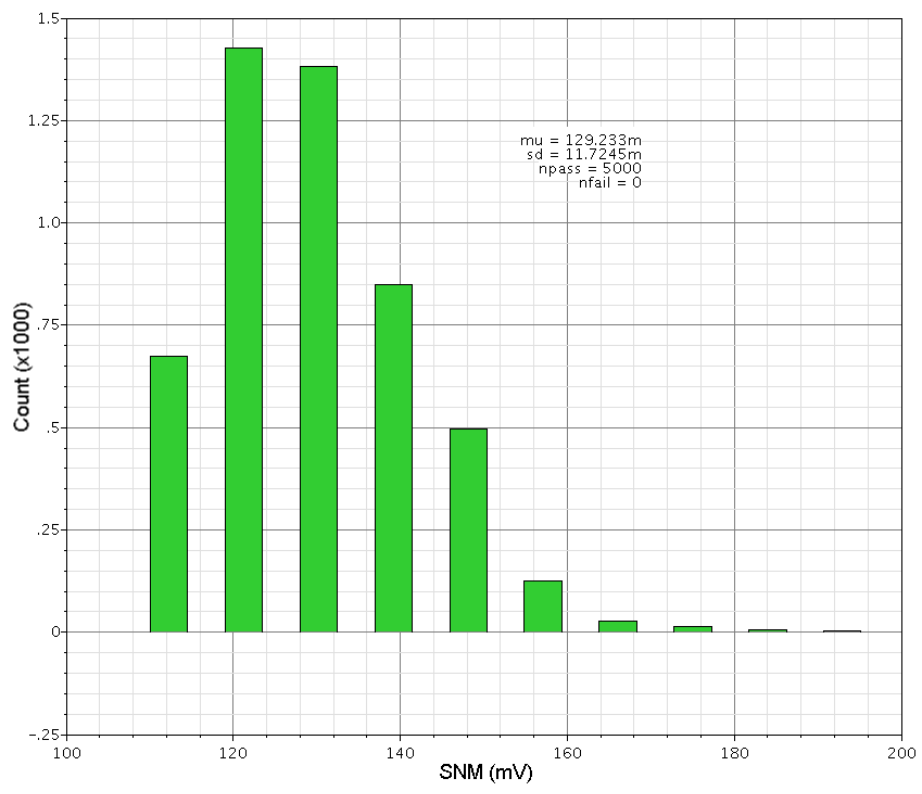

Figure A.8: Histogram for the single-ended write operation of the traditional $8 \mathrm{~T}$ cell. $V_{D D}$ is $300 \mathrm{mV}$ and the temperature is $27^{\circ} \mathrm{C}$. 


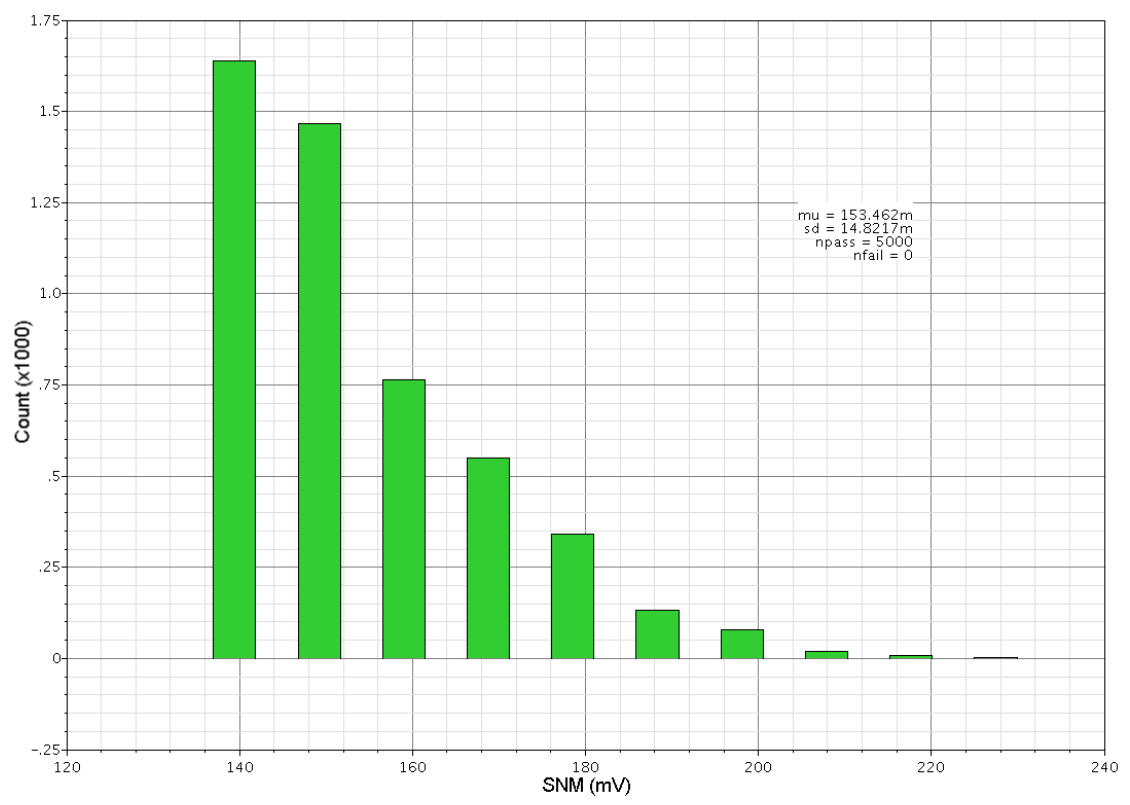

Figure A.9: Histogram for the write operation of the traditional $8 \mathrm{~T} /$ standard $6 \mathrm{~T}$ cell. $V_{D D}$ is $300 \mathrm{mV}$ and the temperature is $27^{\circ} \mathrm{C}$.

Table A.8: 2-cell Configuration

Write SNM
\begin{tabular}{|l|c|c|c|c|}
\hline$V_{D D}$ & $\mu$ & $\sigma$ & $\mu-4 \sigma$ & $\mu-5 \sigma$ \\
\hline $200 \mathrm{mV}$ & 65.4857 & 11.7298 & 18.5665 & 6.8367 \\
$250 \mathrm{mV}$ & 92.3955 & 13.5898 & 38.0363 & 24.4465 \\
$275 \mathrm{mV}$ & 105.481 & 14.2639 & 48.4254 & 34.1615 \\
$300 \mathrm{mV}$ & 118.291 & 14.8275 & 58.981 & 44.1535 \\
$350 \mathrm{mV}$ & 143.114 & 15.6964 & 80.3284 & 64.632 \\
\hline
\end{tabular}


Table A.9: 4-cell Configuration

\begin{tabular}{|l|c|c|c|c|}
\multicolumn{5}{|c}{ Write SNM } \\
\hline$V_{D D}$ & $\mu$ & $\sigma$ & $\mu-4 \sigma$ & $\mu-5 \sigma$ \\
\hline $200 \mathrm{mV}$ & 65.1295 & 11.7474 & 18.1399 & 6.3925 \\
$250 \mathrm{mV}$ & 92.2802 & 13.6036 & 37.8658 & 24.2622 \\
$275 \mathrm{mV}$ & 105.417 & 14.2737 & 48.3222 & 34.0485 \\
$300 \mathrm{mV}$ & 118.254 & 14.8343 & 58.9168 & 44.0825 \\
$350 \mathrm{mV}$ & 143.102 & 15.6998 & 80.3028 & 64.603 \\
\hline
\end{tabular}

Table A.10: 8-cell Configuration

Write SNM
\begin{tabular}{|l|c|c|c|c|}
\hline$V_{D D}$ & $\mu$ & $\sigma$ & $\mu-4 \sigma$ & $\mu-5 \sigma$ \\
\hline $200 \mathrm{mV}$ & 64.4401 & 11.7793 & 17.3229 & 5.5436 \\
$250 \mathrm{mV}$ & 92.0519 & 13.6304 & 37.5303 & 23.8999 \\
$275 \mathrm{mV}$ & 105.288 & 14.2931 & 48.1156 & 33.8225 \\
$300 \mathrm{mV}$ & 118.254 & 14.8343 & 58.9168 & 44.0825 \\
$350 \mathrm{mV}$ & 143.077 & 15.7065 & 80.251 & 64.5445 \\
\hline
\end{tabular}


Table A.11: Traditional 8T

Write SNM

\begin{tabular}{|l|c|c|c|c|}
\hline$V_{D D}$ & $\mu$ & $\sigma$ & $\mu-4 \sigma$ & $\mu-5 \sigma$ \\
\hline $200 \mathrm{mV}$ & 104.893 & 17.1443 & 36.3158 & 19.1715 \\
$250 \mathrm{mV}$ & 129.194 & 15.7165 & 66.328 & 50.6115 \\
$300 \mathrm{mV}$ & 153.462 & 14.8217 & 94.1752 & 79.3535 \\
$350 \mathrm{mV}$ & 177.011 & 14.314 & 119.755 & 105.441 \\
\hline
\end{tabular}

Table A.12: Traditional 8T-SE

Single Ended Write SNM

\begin{tabular}{|l|c|c|c|c|}
\hline$V_{D D}$ & $\mu$ & $\sigma$ & $\mu-4 \sigma$ & $\mu-5 \sigma$ \\
\hline $200 \mathrm{mV}$ & 82.4992 & 9.7812 & 43.3744 & 33.5932 \\
$250 \mathrm{mV}$ & 106.214 & 11.171 & 61.53 & 50.359 \\
$275 \mathrm{mV}$ & 117.755 & 11.4396 & 71.9966 & 60.557 \\
$300 \mathrm{mV}$ & 129.233 & 11.7245 & 82.335 & 70.6105 \\
$350 \mathrm{mV}$ & 152.115 & 12.412 & 102.467 & 90.055 \\
\hline
\end{tabular}


Table A.13: Standard 6T

Write SNM

\begin{tabular}{|l|c|c|c|c|}
\hline$V_{D D}$ & $\mu$ & $\sigma$ & $\mu-4 \sigma$ & $\mu-5 \sigma$ \\
\hline $200 \mathrm{mV}$ & 104.893 & 17.1443 & 36.3158 & 19.1715 \\
$250 \mathrm{mV}$ & 129.194 & 15.7165 & 66.328 & 50.6115 \\
$300 \mathrm{mV}$ & 153.462 & 14.8217 & 94.1752 & 79.3535 \\
$350 \mathrm{mV}$ & 177.011 & 14.314 & 119.755 & 105.441 \\
\hline
\end{tabular}




\section{A.3 Read Operation}

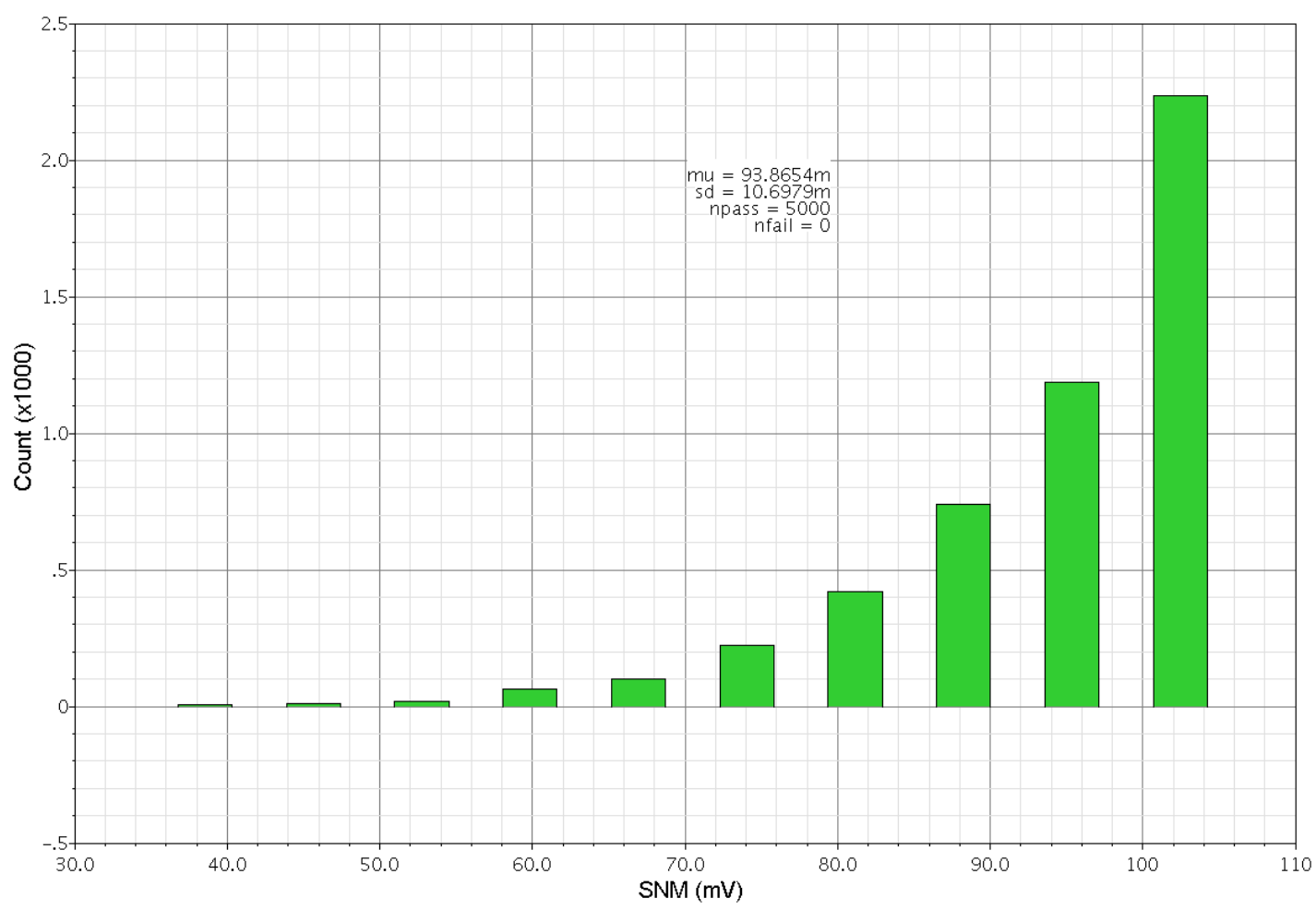

Figure A.10: Histogram for the read operation of the 8-cell block configuration. $V_{D D}$ is $300 \mathrm{mV}$ and the temperature is $27^{\circ} \mathrm{C}$. Only the 8-cell configuration is included because all configurations have very similar distributions. This is similarly true for all sub-threshold operating voltages. 


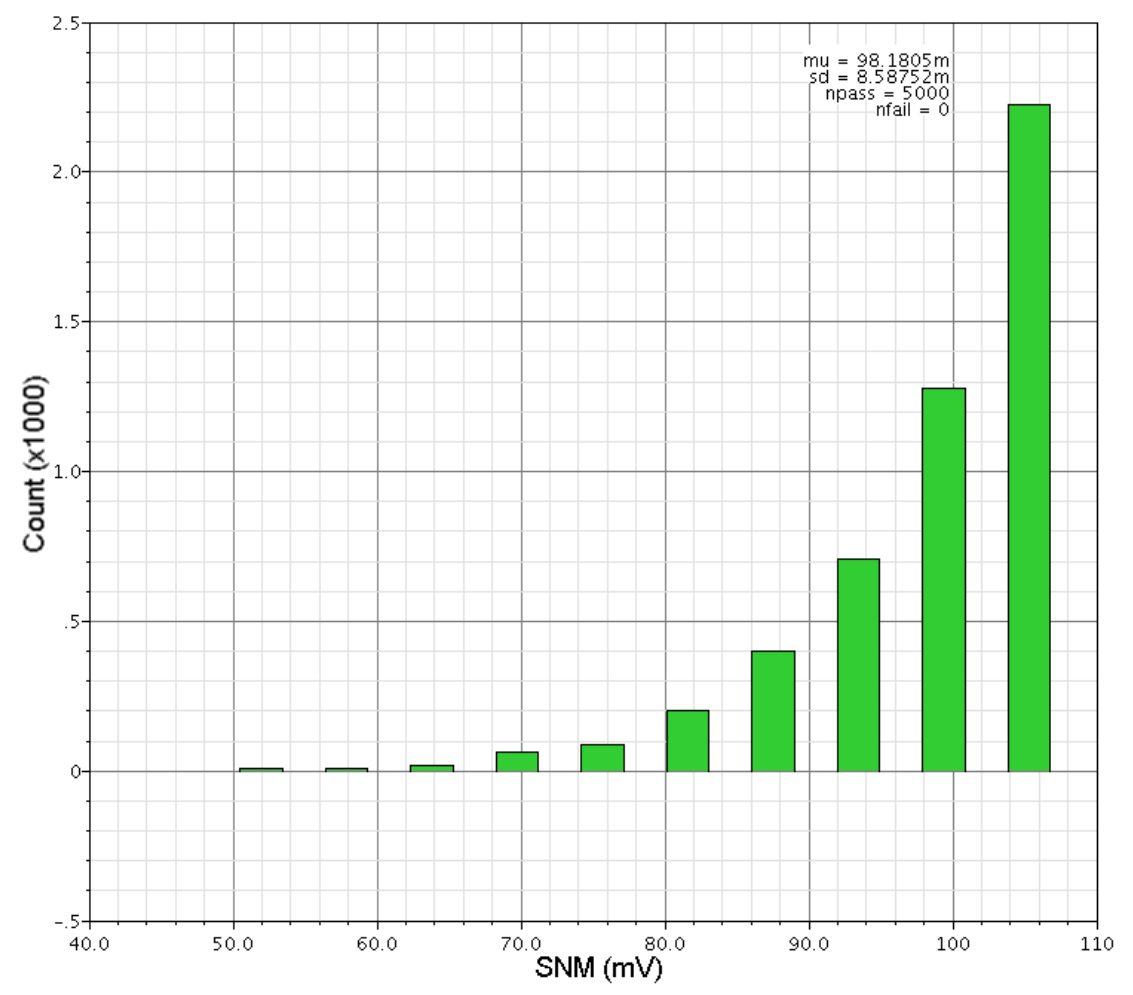

Figure A.11: Histogram for the read operation of the traditional $8 \mathrm{~T}$ cell. $V_{D D}$ is $300 \mathrm{mV}$ and the temperature is $27^{\circ} \mathrm{C}$.

Table A.14: 1-cell Configuration

Read SNM

\begin{tabular}{|l|c|c|c|c|}
\hline$V_{D D}$ & $\mu$ & $\sigma$ & $\mu-4 \sigma$ & $\mu-5 \sigma$ \\
\hline $200 \mathrm{mV}$ & 48.1531 & 10.7043 & 5.3359 & -5.3684 \\
$250 \mathrm{mV}$ & 72.4415 & 10.0431 & 32.2691 & 22.226 \\
$275 \mathrm{mV}$ & 84.3788 & 9.85534 & 44.95744 & 35.1021 \\
$300 \mathrm{mV}$ & 96.2311 & 9.72389 & 57.33554 & 47.61165 \\
$350 \mathrm{mV}$ & 119.704 & 9.53813 & 81.55148 & 72.01335 \\
\hline
\end{tabular}




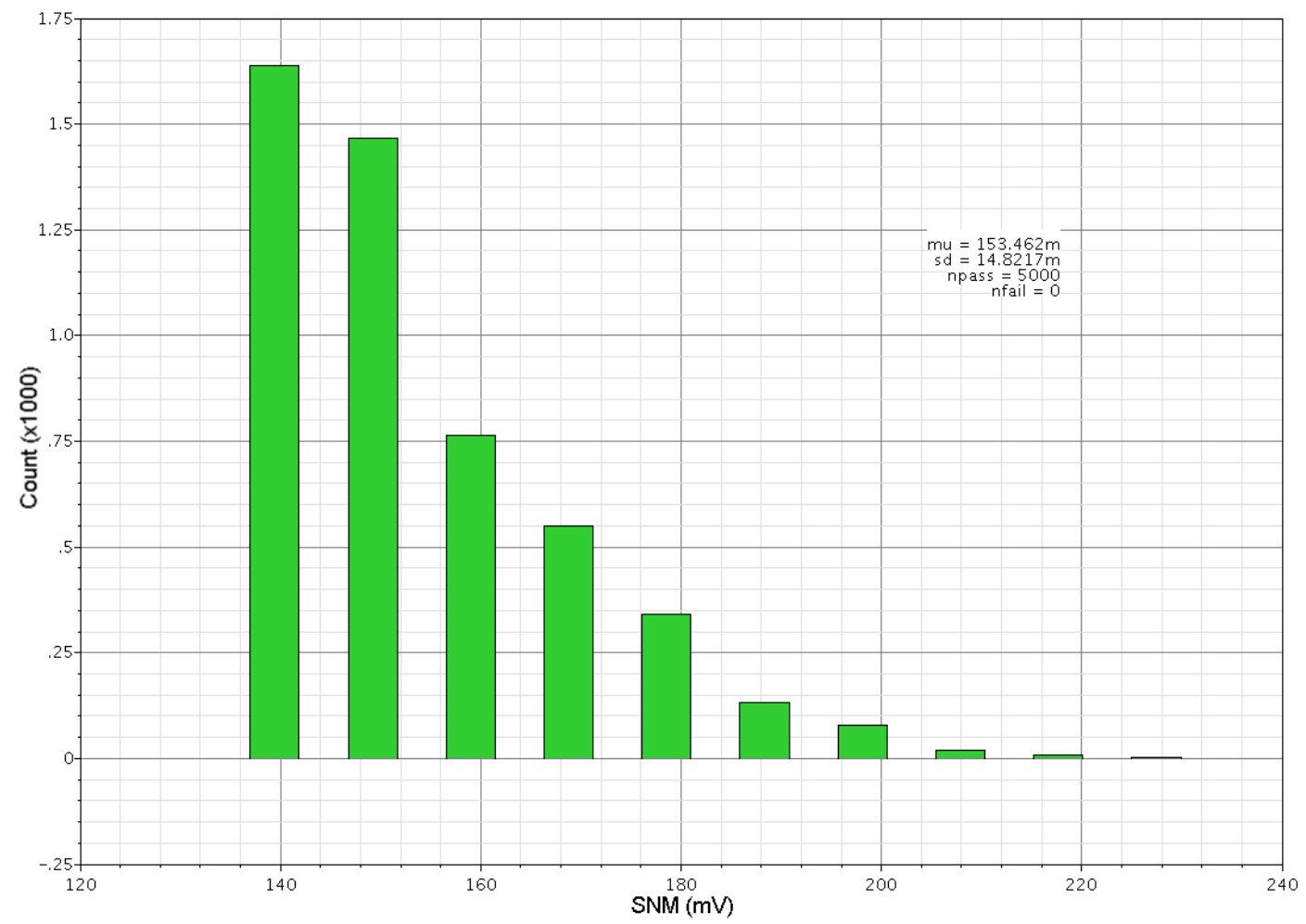

Figure A.12: Histogram for the read operation of the standard $6 \mathrm{~T}$ cell. $V_{D D}$ is 300 $\mathrm{mV}$ and the temperature is $27^{\circ} \mathrm{C}$.

Table A.15: 2-cell Configuration

Read SNM

\begin{tabular}{|l|c|c|c|c|}
\hline$V_{D D}$ & $\mu$ & $\sigma$ & $\mu-4 \sigma$ & $\mu-5 \sigma$ \\
\hline $200 \mathrm{mV}$ & 45.7816 & 12.0834 & -2.552 & -14.6354 \\
$250 \mathrm{mV}$ & 71.7763 & 10.3138 & 30.5211 & 20.2073 \\
$275 \mathrm{mV}$ & 83.8431 & 10.2638 & 42.7879 & 32.5241 \\
$300 \mathrm{mV}$ & 96.6968 & 9.37254 & 59.20664 & 49.8341 \\
$350 \mathrm{mV}$ & 119.488 & 9.64976 & 80.88896 & 71.2392 \\
\hline
\end{tabular}


Table A.16: 4-cell Configuration

Read SNM

\begin{tabular}{|l|c|c|c|c|}
\hline$V_{D D}$ & $\mu$ & $\sigma$ & $\mu-4 \sigma$ & $\mu-5 \sigma$ \\
\hline $200 \mathrm{mV}$ & 45.5673 & 11.5122 & -0.4815 & -11.9937 \\
$250 \mathrm{mV}$ & 70.5921 & 10.7534 & 27.5785 & 16.8251 \\
$275 \mathrm{mV}$ & 82.9585 & 10.5468 & 40.7713 & 30.2245 \\
$300 \mathrm{mV}$ & 95.1573 & 10.2375 & 54.2073 & 43.9698 \\
$350 \mathrm{mV}$ & 119.074 & 9.85372 & 79.65912 & 69.8054 \\
\hline
\end{tabular}

Table A.17: 8-cell Configuration

\begin{tabular}{|l|c|c|c|c|}
\multicolumn{5}{|c|}{ Read SNM } \\
\hline$V_{D D}$ & $\mu$ & $\sigma$ & $\mu-4 \sigma$ & $\mu-5 \sigma$ \\
\hline $200 \mathrm{mV}$ & 42.955 & 12.2036 & -5.8594 & -18.063 \\
$250 \mathrm{mV}$ & 68.641 & 11.3526 & 23.2306 & 11.878 \\
$275 \mathrm{mV}$ & 81.3352 & 10.9978 & 37.344 & 26.3462 \\
$300 \mathrm{mV}$ & 95.1245 & 10.2317 & 54.1977 & 43.966 \\
$350 \mathrm{mV}$ & 118.313 & 10.191 & 77.549 & 67.358 \\
\hline
\end{tabular}

Table A.18: Traditional 8T

Read SNM

\begin{tabular}{|l|c|c|c|c|}
\hline$V_{D D}$ & $\mu$ & $\sigma$ & $\mu-4 \sigma$ & $\mu-5 \sigma$ \\
\hline $200 \mathrm{mV}$ & 51.8192 & 8.51484 & 17.75984 & 9.245 \\
$250 \mathrm{mV}$ & 75.085 & 8.53884 & 40.92964 & 32.3908 \\
$275 \mathrm{mV}$ & 86.6686 & 8.57489 & 52.36904 & 43.79415 \\
$300 \mathrm{mV}$ & 98.1805 & 8.58752 & 63.83042 & 55.2429 \\
$350 \mathrm{mV}$ & 121.116 & 8.67459 & 86.41764 & 77.74305 \\
\hline
\end{tabular}


Table A.19: Standard 6T

\begin{tabular}{|c|c|c|c|c|}
\hline \multicolumn{5}{|c|}{ Read SNM } \\
\hline$V_{D D}$ & $\mu$ & $\sigma$ & $\mu-4 \sigma$ & $\mu-5 \sigma$ \\
\hline $200 \mathrm{mV}$ & 16.0453 & 23.4658 & -77.8179 & -101.284 \\
\hline $250 \mathrm{mV}$ & 26.2094 & 23.139 & -66.3466 & -89.4856 \\
\hline $275 \mathrm{mV}$ & 31.6987 & 22.8435 & -59.6753 & -82.5188 \\
\hline $300 \mathrm{mV}$ & 37.3789 & 22.4316 & -52.3475 & -74.7791 \\
\hline $350 \mathrm{mV}$ & 49.3672 & 21.0806 & -34.9552 & -56.0358 \\
\hline
\end{tabular}




\section{Appendix B}

\section{Copyright Permissions}

\section{B.1 Intel Copyright Permission}

Automatic permission to Sukneet Basuta:

Please consider this message a grant of permission but only in the manner you describe in your request.

This message provides a limited copyright license, the scope of which is implied by your original request.No other intellectual property licenses (trademark, patent or otherwise) are granted. All materials are provided by Intel 'AS IS,' with no warranties whatsoever. Any disputes over the license granted herein will be governed by California law.

Thank you for visiting our site. Intel Corporation.

Regards,

Intel Copyright Permission Department.

\section{B.2 IEEE Copyright Permission}

Thesis / Dissertation Reuse

The IEEE does not require individuals working on a thesis to obtain a formal reuse license, however, you may print out this statement to be used as a permission grant:

Requirements to be followed when using any portion (e.g., figure, graph, table, or textual material) of an IEEE copyrighted paper in a thesis: 
1) In the case of textual material (e.g., using short quotes or referring to the work within these papers) users must give full credit to the original source (author, paper, publication) followed by the IEEE copyright line (C)2011 IEEE.

2) In the case of illustrations or tabular material, we require that the copyright line (C) [Year of original publication] IEEE appear prominently with each reprinted figure and/or table.

3) If a substantial portion of the original paper is to be used, and if you are not the senior author, also obtain the senior authors approval.

Requirements to be followed when using an entire IEEE copyrighted paper in a thesis:

1) The following IEEE copyright/ credit notice should be placed prominently in the references: (C) [year of original publication] IEEE. Reprinted, with permission, from [author names, paper title, IEEE publication title, and month/year of publication]

2) Only the accepted version of an IEEE copyrighted paper can be used when posting the paper or your thesis on-line.

3) In placing the thesis on the author's university website, please display the following message in a prominent place on the website: In reference to IEEE copyrighted material which is used with permission in this thesis, the IEEE does not endorse any of [university/educational entity's name goes here]'s products or services. Internal or personal use of this material is permitted. If interested in reprinting/republishing IEEE copyrighted material for advertising or promotional purposes or for creating new collective works for resale or redistribution, please go to http://www. ieee.org/ publications_standards/publications/rights/rights_link.html to learn how to obtain a License from RightsLink.

If applicable, University Microfilms and/or ProQuest Library, or the Archives of Canada may supply single copies of the dissertation. 